This document is downloaded from DR-NTU (https://dr.ntu.edu.sg) Nanyang Technological University, Singapore.

lon doping behavior of stöber

silica-nanosynthesis and application

Song, Xiaohui

2016

https://hdl.handle.net/10356/66042

https://doi.org/10.32657/10356/66042 


\section{Ion Doping Behavior of Stöber Silica-Nanosynthesis and Application}

SONG XIAOHUI

School of Physical and Mathematical Sciences

A thesis submitted to the Nanyang Technological University in partial fulfillment of the requirement for the degree of Doctor of Philosophy

Singapore

Aug 2015 
Advisor: $\quad$ Associate Professor Chen Hongyu (NTU)

Co-advisor: Dr. Wei Jun (SIMTech)

Associate Professor Pumera Martin (NTU) 


\section{Acknowledgement}

Here I would like to need show my appreciation to these people who have supported me during my pursuit of the degree of Doctor of Philosophy. It is a long way for me, my supervisor, and my family.

Firstly, I want to thank my professor Dr. Chen Hongyu. He is not only a good advisor but also a nice friend. I still remember the when I was a freshman in the lab and I was nervous so I made many mistakes in the lab. He was very kind and patient to talk to me, let me know what a good attitude should to be towards research, and showed me how I should design my experiments. According the past four years Professor Chen has spent so much time on me and my projects. The most valuable thing to research is that attitude and methodology are more important than knowledge.

I also need to thanks all my group members for the help and advisement during the past four years. It must be difficult for me without your help. My friends out office are all great to help me relax, taking care me in life. Thanks NTU graduate research scholar support me for research funding.

Finally, I want to give my appreciation to my parents. They are the most important persons to me and thank you for your support in my daily life. I am proud of them and would always love them. 


\section{Contents}

Acknowledgement ……………………………………………………….III

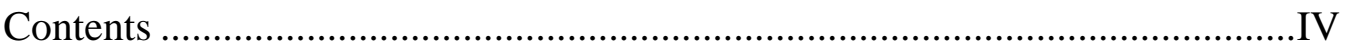

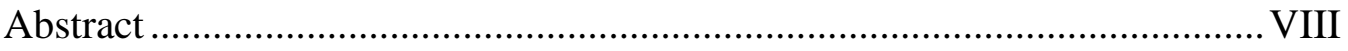

Chapterl Introduction ................................................................................ 11

1.1 Ion effects on nanosynthesis. ........................................................... 11

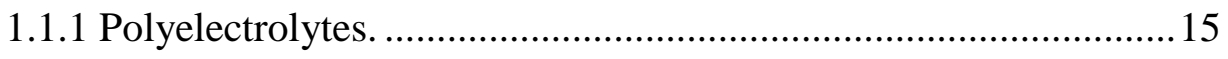

1.1.2 DNA and RNA precipitation. ......................................................18

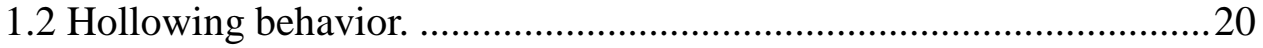

1.2.1 Selective etching. ........................................................................2

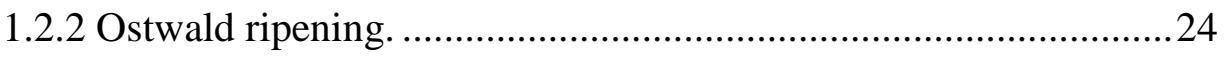

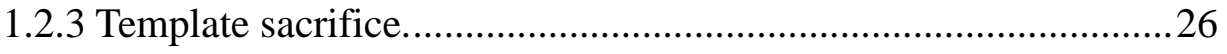

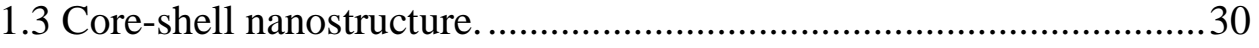

1.3.1 Nucleation and surface deposition. ................................................. 32

1.3.2 Double-domain nanoparticle morphology........................................35

1.3.3 Metal NP@ oxide core-shell nanoparticle......................................36

1.3.4 Metal NP@polymer core-shell nanoparticle..................................39

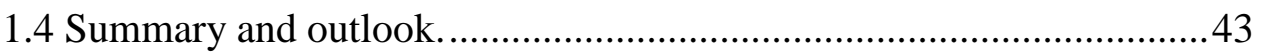

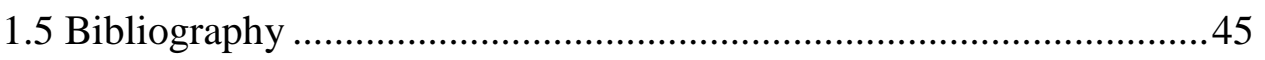

Chapter 2 Synthesis and Etching Study of Stöber Silica Nanoparticles .............51

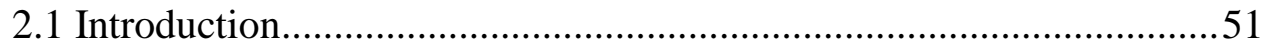

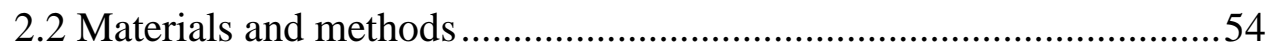

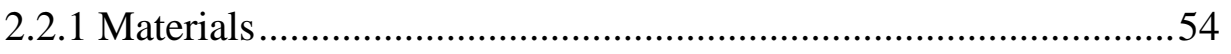

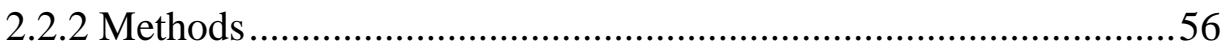

2.3 Results and discussion ...............................................................5

2.3.1 Synthesis and etching ..................................................................61

2.3.2 Understanding silica dissolution in water ........................................64

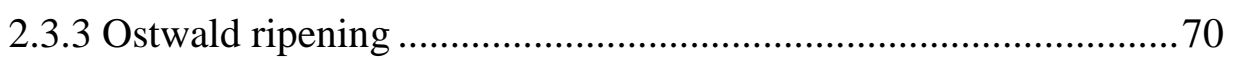

2.3.4 Hypothesis of silica formation ...................................................... 71

2.3.5 Analysis of silica composition........................................................ 
2.3.6 Synthesis of Stöber silica under different salt present .81

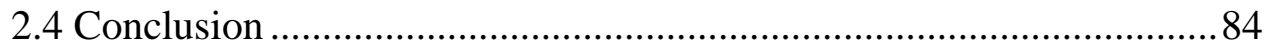

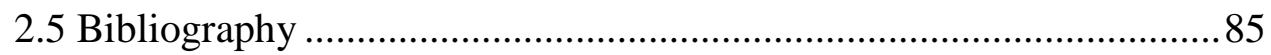

Chapter 3 Exploring the Ion Pairing Formation Mechanism into Application .. 88

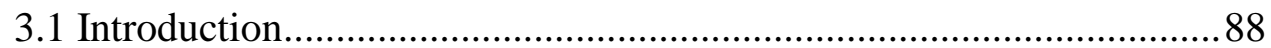

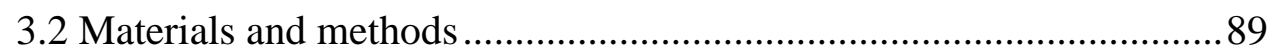

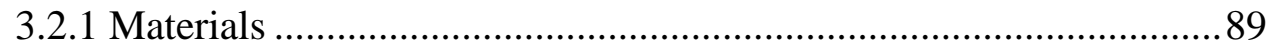

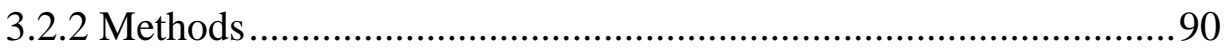

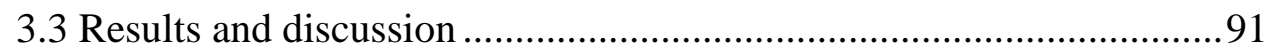

3.3.1 Rational synthesis of silica nanostructures ................................92

3.3.2 Doping heavy metals inside silica ..............................................94

3.3.3 Doping organic molecules and drugs inside silica ........................97

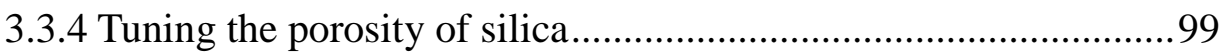

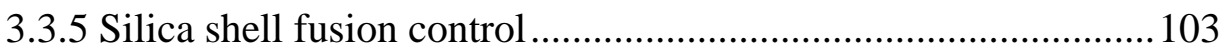

3.3.6 Synthesis of silica in pure water.............................................. 105

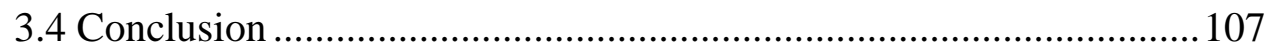

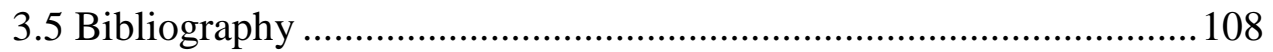

Chapter 4 Exploring Ion Pairing Theory into Metal-Silica Janus synthesis .... 113

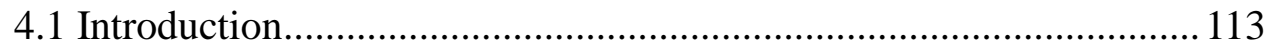

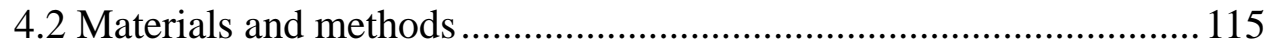

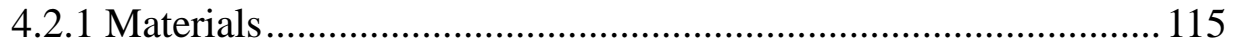

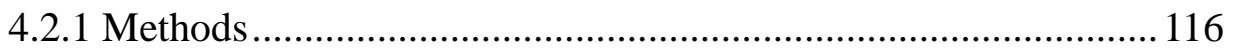

4.2.3 Seed concentration .............................................................. 121

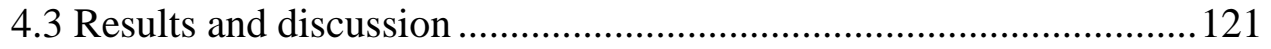

4.3.1 Iron oxide coating silica in reverse emulsion ...............................122

4.3.2 Hydrophilic Au encapsulation in reverse emulsion ................... 125

4.3.3 Mechanism study................................................................ 128

4.3.4 Silica synthesis and etching....................................................134

4.3.5 Au NP coating by stöber method...............................................136

4.3.6 Salt addition in reverse emulsion synthesis................................139

4.3.7 Generality for Janus synthesis............................................... 143

4.3.8 Design nanostructure by Janus fabrication................................. 144 


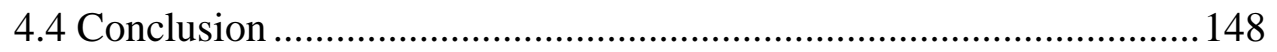

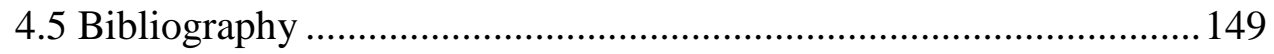

Chapter 5 Synthesis and Mechanism Investigation of the Metal@PSPAA

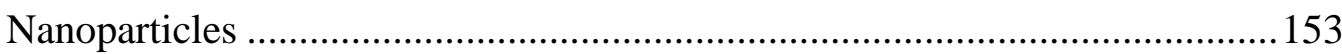

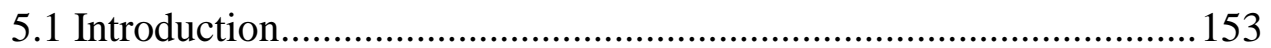

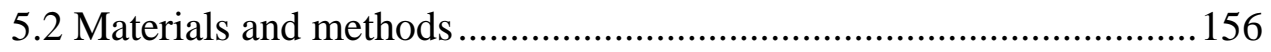

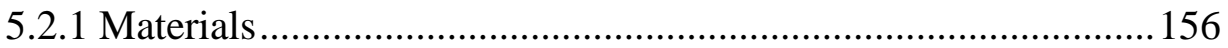

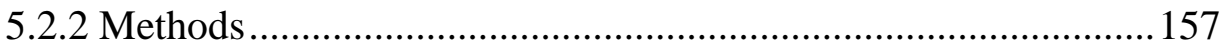

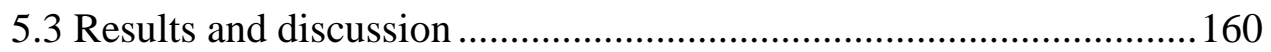

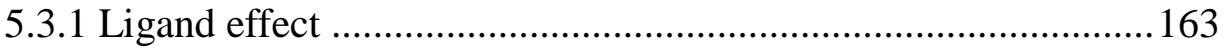

5.3.2 PSPAA assembly mechanism onto Au nanoparticle ...................166

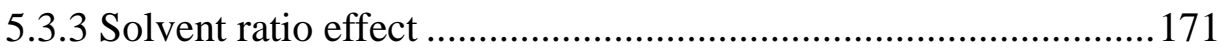

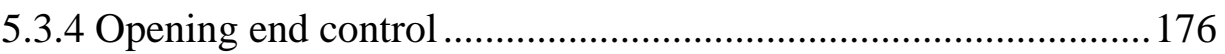

5.3.5 PSPAA shell mobility control.................................................. 177

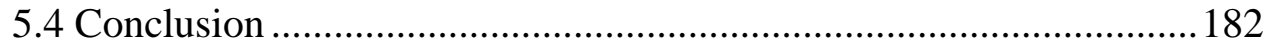

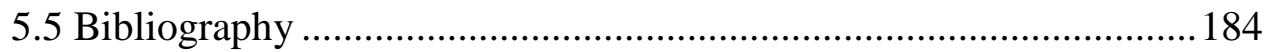

Chapter 6 Homo- and Co-Polymerization of Polysytrene-Block-Poly(Acrylic

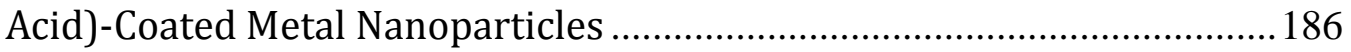

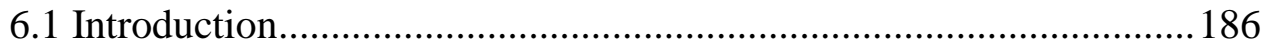

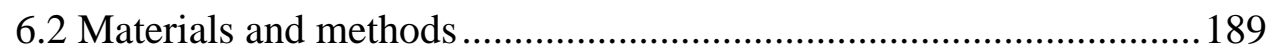

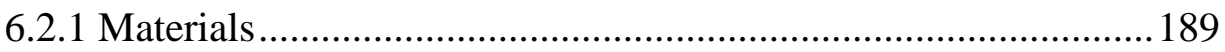

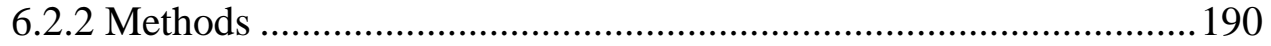

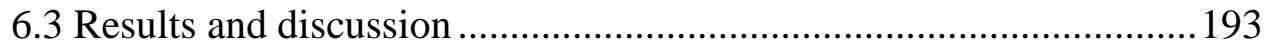

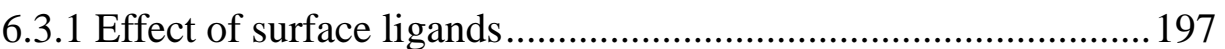

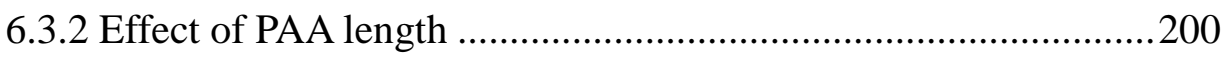

6.3.3 Selection of the preparative conditions .....................................203

6.3.4 "Homo-polymers" of nanorods and nanowires ...........................206

6.3.5 Random “copolymers” of NPs ...................................................208

6.3.6 "Copolymers" of NPs with cylinders/vesicles ............................213

6.3.7 “Block copolymer” of NWs with NPs ......................................216

6.3.8 Random versus block "copolymers" ..........................................218

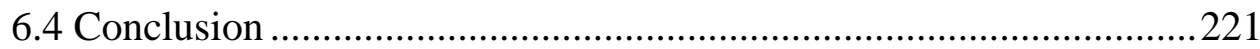


6.5 Bibliography

Chapter 7 Summary and Outlook

7.1 Summary .229

7.1.1 Silica formation mechanism and application .230

7.1.2 Metal NPs@PSPAA design and synthesis .233

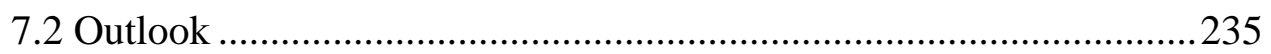

7.2.1 Ion doping behavior of $\mathrm{Cu}_{2} \mathrm{O}$ nanoparticle .............................237

7.2.2 Control polymer shell porosity.................................................243

7.2.3 Design novel structures for catalyst-proposed application. ........246

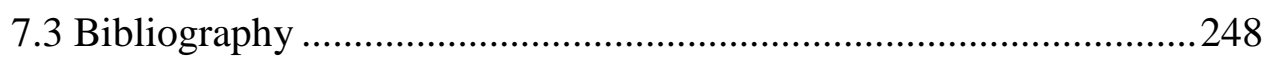

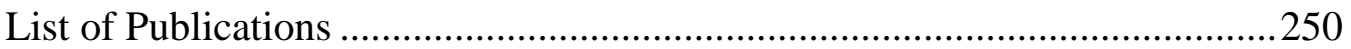




\section{Abstract}

The research work presented in this thesis focused on the fundamental study of nanostructure synthesis, such as the colloidal synthesis control, the hollowing behavior mechanism study, the fabrication control, the silica or polymer shell coating control, polymerization control, as well as primary application based on the mechanism study, such as the transition metal doping control, the porosity control, as well as the drug loading control in core-shell nanostructures. We systematically study the Stöber silica nucleation and deposition process under kinetics and thermodynamics theory. On one hand, the ion doping behavior is critical to silica nucleation and deposition, which leads to the chemical composition difference between the inner layer and the outmost layer of silica nanoparticles. On the other hand, the ion-doping control can help us to design novel nanostructures, and it is possible to be applied to other metal oxides. The whole research open new windows to the future design and application in other areas, such as biosensors, drug delivery, energy conversion, and so on.

The Stöber silica etching behavior attracted our attention, and we started to study the reason of selective etching behavior for silica nanoparticles (Chapter 2). It is not because of etching but because of ion doping behavior during the synthesis which leads to the composition difference between the inner layer and the outmost layer. After many control experiments the surface redeposition 
mechanism, and the Ostwald ripening mechanism have been ruled out, which were usually applied to explain metal oxide hollowing behavior. The new breakthrough helps us to view silica as counter ions trapped inside which is critical to porosity. The elemental analysis, EDX line scan and TEM provide evidence to above hypothesis.

Based on the ion pairing mechanism during silica nucleation and deposition we designed many experiments to get novel silica nanostructures (Chapter 3). By tuning the solvent ratio, the ions concentration, and the acid incubation time length we can control the ion doping level inside silica nanoparticles, which will give many different kinds of novel structures, such as hard-soft-hard silica, lower porous silica, and transition metal doped silica. Besides, we can dope positive charged organic molecules inside silica nanoparticle, such as dyes, drugs, and so on. The above synthesis control is important to silica application in biosensors, drug delivery, catalysts, and battery.

The deeper study was conducted for the silica coating metal nanoparticles under the ion pairing mechanism of silica formation. We found that the metal nanoparticles encapsulation can be adjusted by tuning solvent ratio (Chapter 4), which means that ion doping difference can affect the silica nucleation and deposition on the nanoparticle surface. We can tune the hydrophilic nanoparticle coating from full encapsulation to partially encapsulation by solvent ratio control in Stöber system/reverse emulsion system. Interestingly, we can also control the 
hydrophobic nanoparticle encapsulation from concentric to eccentric, and Janus nanoparticle by the similar solvent ratio control in reverse emulsion system. It is a simple, general method to synthesize Janus nanoparticles by ion doping control during the metal nanoparticle coating process.

In Chapter 5, another type of core-shell structure, metal nanoparticle coated by poly(styrene)-block-poly(acrylic acid) (PSPAA) shell was studied. Firstly, we studied the polymer nucleation and deposition on the nanoparticle surface, and then control the solvent ratio to synthesize different kinds of core-shell nanoparticles, such as concentric nanoparticles, multi island core-shell nanoparticles, as well as Janus nanoparticles. We also study the mobility of the polymer shell by tuning the solvent ratio, the temperature, as well as the other parameters. All these studies provide a simple way to synthesis core-shell (Metal NP@PSPAA) nanostructures.

Finally, the Metal NP@PSPAA core-shell nanoparticles were used to fabricate novel nanostructures (Chapter 6). We have successfully controlled the nanoparticle polymerization according to change the charge repulsion of the Metal NP@PSPAA monomer. Co-, homo-, and block polymerization of the nanoparticles have been studied while tuning different parameters, such as the solvent ratio (water/DMF, or THF), the seed ratio, the polymer chain length, and so on. This method is easy to control and very simple which opens new window for novel nanostructure design. 


\section{Chapter1 Introduction}

Every shape has its reason of formation in nanomaterials. The mechanism study is to analyze the possibilities of a typical nanostructure formation. The mechanism study is usually combined by design control experiments, characterization, as well as the traditional data compare, which finally provides a logical explain based on kinetic and thermodynamic theory.

Nanostructure design and synthesis is critical to the material science, such as the application in biosensors, ${ }^{1}$ the catalysts, ${ }^{2}$ battery, ${ }^{3}$ as well as drug delivery ${ }^{4}$. The structure properties of the nanostructures are critical to its application. So the nanostructure control is now a hot topic in material science. Here we will review the Stöber hollow silica formation and control mechanism, the ions effects on the nanosynthesis. We hope it can give a big picture for the nanostructures synthesis based on the ion/ion doping effects.

\subsection{Ion effects on nanosynthesis.}

In an inorganic chemical reaction, the ions are a kind of normal existent state in most of the systems. The main reason is that most of the precursors are salt, or polyelectrolyte, while the final products show high intensity of surface charge, such as most the oxide nanoparticles, ligand or surfactant stabilized nanoparticles, ${ }^{5}$ as well as polymers there. According to control the ion concentration, the diffusion speed, and the ion exchange we can control the final 
morphologies, and properties of the final nanostructures.
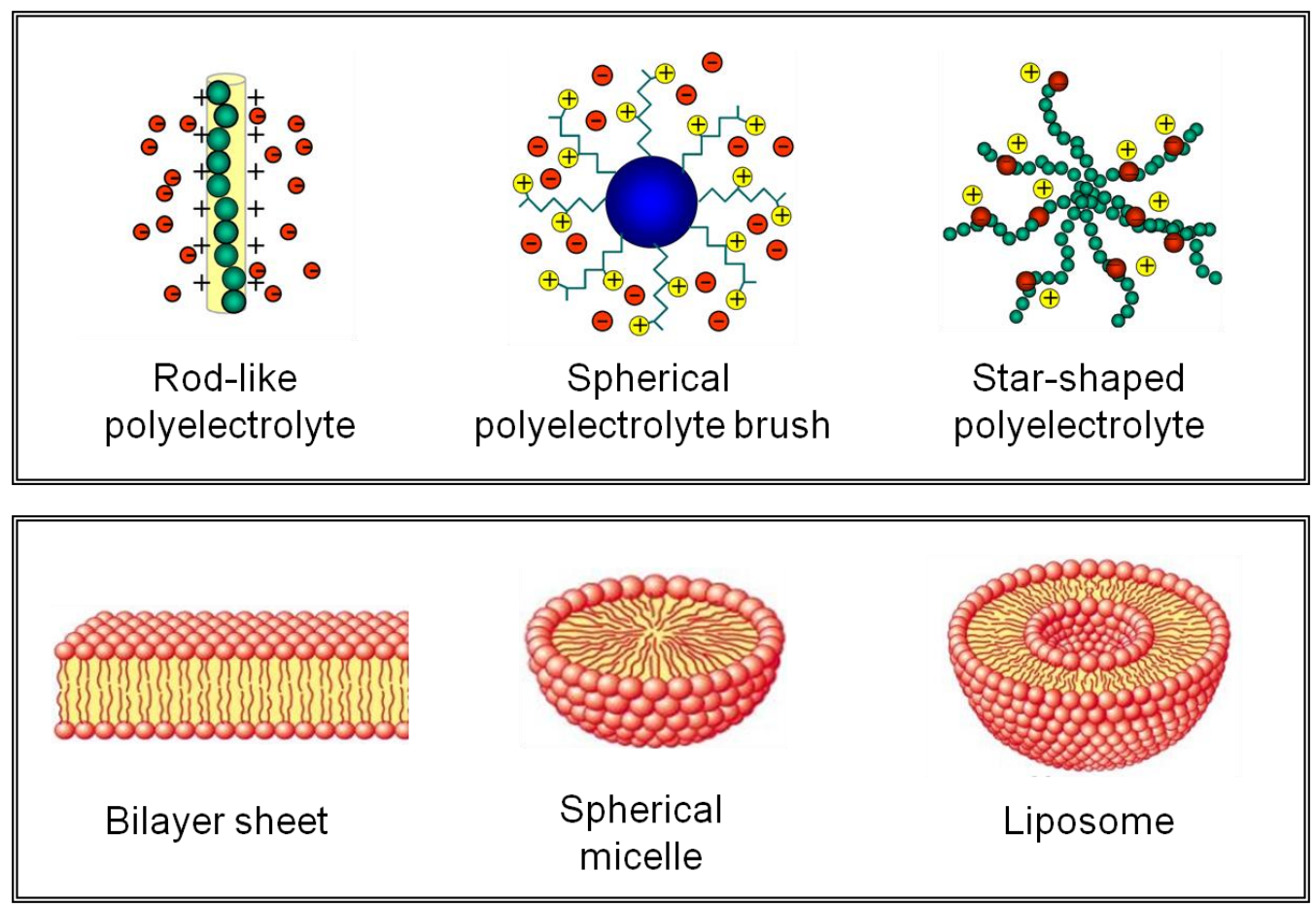

Figure 1.1. The scheme of polyelectrolyte fabrication under different condition after ion doping. The morphology is from cylinder to vesicle. Reprinted with permission from ref 4. Copyright 2009 from American Chemical Society.

For the ion effects on the nanosynthesis, there are many studies started from polyelectrolyte about the ions effects on its polymerization, nucleation, precipitation, and deposition process. ${ }^{1}$ Another hot area is DNA, RNA study, which have been linked with the formation of nanomaterials. ${ }^{2}$ The ions show broad application and effects on the DNA, RNA segregation, or nanostructure formation. ${ }^{3}$ According to figure 1.1 we can see there are many different kinds of morphologies during the polyelectrolyte doping with counter ions, which means that the counter ions can affect the polyelectrolyte micelle formation, as well as 
the final morphologies, such as bilayer sheet, spherical nanoparticles, vesicle, and so on. All these kinds fabrication is critical to its potential application.

Typically ion doping effects on nanostructure morphology have been digested in many different research groups. ${ }^{6}$ Ions inside the solution can affect reaction environment by affecting many different parameters, such as the polarity of the solvent, the solubility of the product/precursor, the catalysts performance inside the solution, and the ligand properties (solubility, stability, as well as the selectivity). For example, ion doped $\mathrm{TiO}_{2}$ nanomaterials have been studied many years for the unique advantages. ${ }^{6}$ The optical response of any material is largely determined by its underlying electronic structure. The electronic properties of a material are closely related to its chemical composition (chemical nature of the bonds between the atoms or ions), its atomic arrangement, and its physical dimension (confinement of carriers) for nanometer-sized materials. The chemical composition of $\mathrm{TiO}_{2}$ can be altered by doping. Specifically, the metal (titanium) or the nonmetal (oxygen) component can be replaced in order to alter the material's optical properties. It is desirable to maintain the integrity of the crystal structure of the photocatalytic host material and to produce favorable changes in electronic structure. It appears easier to substitute the $\mathrm{Ti}^{+}$cations in $\mathrm{TiO}_{2}$ with other transition metals, and it is more difficult to replace the $\mathrm{O}^{2-}$ anion with other anions due to differences in charge states and ionic radii. The small size of the nanoparticle is beneficial for the modification of the chemical composition of 
$\mathrm{TiO}_{2}$ due to the higher tolerance of the structural distortion than that of bulk materials induced by the inherent lattice strain in nanomaterials. ${ }^{6}$ As shown in figure 1.2, it is clear to find that when $\mathrm{TiO}_{2}$ was doped with $\mathrm{V}, \mathrm{Cr}, \mathrm{Mn}, \mathrm{Fe}$, or Co, an electron occupied level formed and the electrons were localized around each dopant. As the atomic number of the dopant increased, the localized level shifted to lower energy. The energy of the localized level due to Co doping was low enough that it lay at the top of the valence band while the other metals produced midgap states. The electrons from the Ni dopant were somewhat delocalized,thus significantly contributing to the formation of the valence band with the $\mathrm{O} p$ and Ti $3 \mathrm{~d}$ electrons. The states due to the $3 \mathrm{~d}$ dopants shifted to a lower energy as the atomic number of the dopant increased.

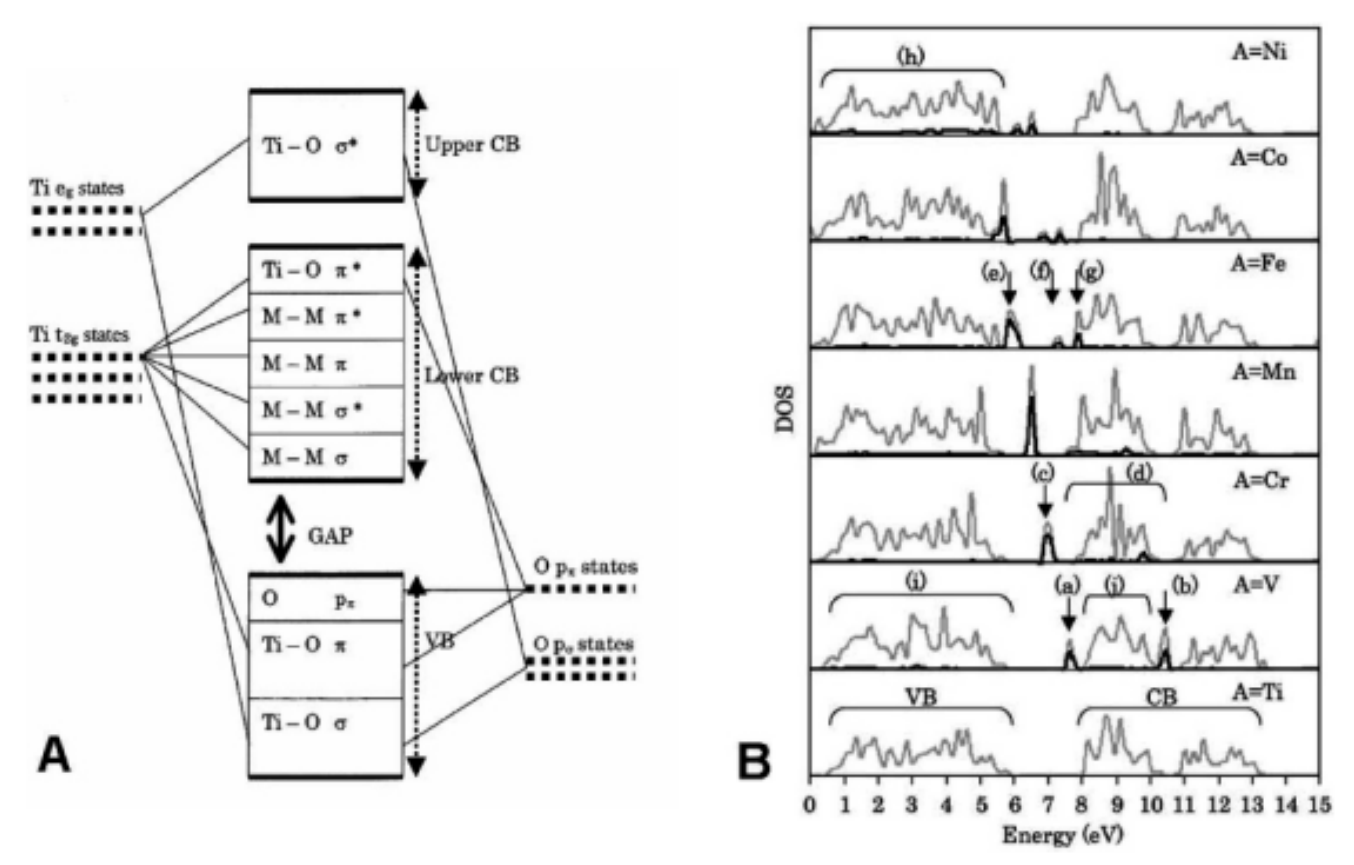

Figure 1.2. (A) Bonding diagram of $\mathrm{TiO}_{2}$. (B) DOS of the metal-doped $\mathrm{TiO}_{2}$ $\left(\mathrm{Ti}_{1-\mathrm{x}} \mathrm{A}_{\mathrm{x}} \mathrm{O}_{2}: \mathrm{A}=\mathrm{V}, \mathrm{Cr}, \mathrm{Mn}, \mathrm{Fe}, \mathrm{Co}\right.$, or Ni). Gray solid lines: total DOS. Black solid lines: dopant's DOS. The states are labeled (a) to (j). Reprinted from J. Phys. 
Chem. Solids 2002, 63, 1909, Copyright 2002, with permission from Elsevier.

\subsubsection{Polyelectrolytes.}

Polyelectrolytes are polymers consisting of monomers having groups, which may ionize in a polar solvent. Normally, polyelectrolytes are polymers bearing dissociable functional groups, which, in polar solvents (water), can dissociate into charged polymer chains (macroions) and small counter ions. ${ }^{5}$ So it is easy to understand that polyelectrolyte shows two properties, salt and polymer properties together. DNA and protein are a kind of natural polyelectrolytes which show high potential in biological study. ${ }^{1}$

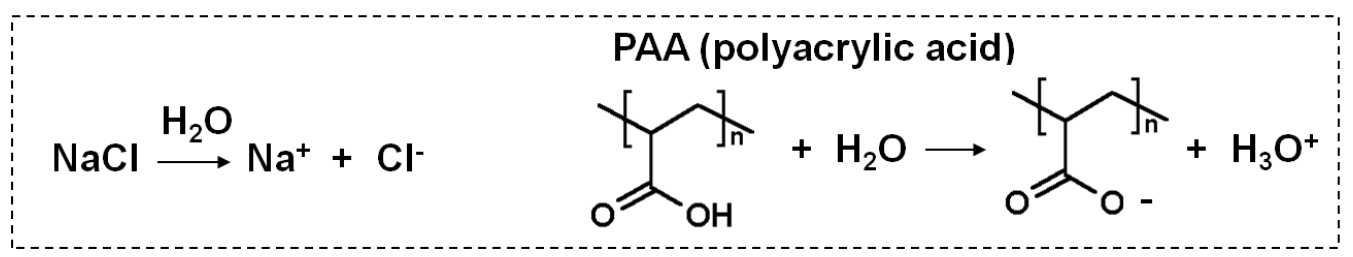

Figure 1.3. The polyacrylic acid showing similar behavior as $\mathrm{NaCl}$ ionizing, which give negative charged polymer, as well as proton. ${ }^{4}$ Reprinted with the permission from ref 4. Copyright 2010 from American Chemical Society.

It is clear to see there are many applications of polyelectrolytes because of its ionic properties combined with polymer properties. Such as polyelectrolyte gel for artificial muscles; hydrophobic modified polyelectrolytes and polyelectrolyte block-copolymers for biomedical applications, ion exchange resins for application in separation, purification, as well as decontamination processes; ${ }^{6}$ application in controlled drug delivery system for experimental; composite 
polyelectrolyte self-assembled nanostructures into biosensor applications; ${ }^{7}$ layer-by-layer thin films combined by polyelectrolyte-based materials for electronic and photonic applications $;^{8}$ polyelectrolyte-Surfactant (ionic polymer) Complexes synthesis and fabrication for industry application. ${ }^{9}$

Firstly, it is reported that the ionic strength and ion concentration can affect the polyelectrolyte solubility and morphology in solvent. ${ }^{10}$ An example is poyacrylic acid (PAA), which has been studied many years. According to figure 1.3, we could find the similar behavior of PAA as that of $\mathrm{NaCl}$. The higher sodium ion concentration in the solution can give more PAA precipitation there, which means that the count ion concentration is critical to PAA solubility: higher counter ion concentration might give higher ion doping while lead to PAA solubility decrease; lower counter ion concentration might give fewer ion doping inside the PAA chains while there is no solubility decrease.

Secondly, the chain length of polyelectrolyte could affect the polymer solubility, morphology, as well as its properties. ${ }^{11}$ As shown in figure 1.4 , it is clear to see a polyelectrolyte expands like polymer swelling in favorable solvent. However, while counter ions were added into solution the polyelectrolyte will aggregate forming a random coil because of counter ion doping to form ion pairs, which means that its solubility could decrease because of counter ion interaction. The solubility will be decrease while polyelectrolyte chain length increase and the reason is longer chain with more negative charges on polyelectrolyte which 
doped more counter ions. The heavier of ion doping could lead to polyelectrolyte solubility decrease dramatically.

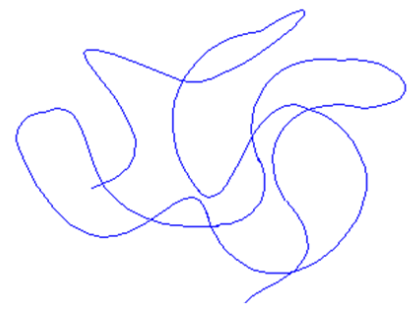

A neutral polymer molecule tangled in a random coil.

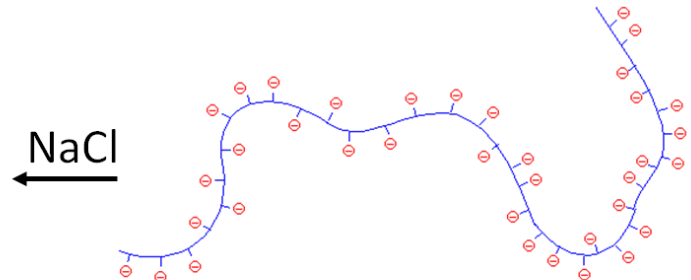

A polyelectrolyte expands because it's like charges repel each other. $\Rightarrow$ more viscous

Figure 1.4. The swelling polymer aggregates after ions addition because of counter ion interaction between polyelectrolyte and ions.

Finally, the solvent quality (polarity) is critical to polyelectrolyte properties, such as solubility, porosity, and ion doping. ${ }^{12}$ There have been reported that polyelectrolyte solubility is higher in polar solvent, such as water, $\mathrm{EtOH}$, and so on. The reason is that the solvate energy is lower compared to non polar solvent. However, while disperse polyelectrolyte into non polar solvent it will aggregate because of higher solvate energy. This process is critical for polyelectrolyte or some polymer application. It has been reported that the shell morphology might be difference when PAA was used to coat $\mathrm{Fe}_{3} \mathrm{O}_{4}$ nanoparticles, where the PAA was dissolved into water/IPA mixture to tune the solvent ratio changing solvent polarity. Higher water content (higher polarity) gives core-shell nanoparticles while lower water content (lower polarity) gives partially encapsulation there.

According to the above review it is clear to see that polyelectrolyte (ionic 
polymer) properties depend on many parameters, such as the polymer chain length, solvent polarity, salt concentration, as well as temperature. ${ }^{5,13,14}$

\subsubsection{DNA and RNA precipitation.}

DNA, RNA, and protein are just kinds of natural polyelectrolyte. ${ }^{15}$ During the past decades people have studied them in depth for the potential application in so many broad areas, such as drug delivery, clinic, as well as biosensors. ${ }^{16}$ In nanoscience, DNA, RNA, and protein molecule can be used to control nanostructure fabrication, ${ }^{17}$ biosensor preparation, ${ }^{18}$ and so on. ${ }^{19}$ The previous strategies for the hetero-assembly is mainly based on the synthesis of anisotropic functionalization of the NPs. Usually NPs were stuck on the surface of substrate via DNA or small molecule linkers, and then functionalized by another linkers. Thus Janus NPs were produced by remove the NPs from substrate, and used for the performance of hetero-assembly. The hetero-assembly is believed to be the development orientation towards the fabrication of future nanodevices. However, these methods developed so far have some disadvantages: more expensive DNA molecules are used; the substrates are often in small scale. To improve the restricted applications, more intelligent technique is highly demanded. The reason is that this kind of natural polyelectrolyte has special properties: charged polymers surface (positive charged center, or negative charged center); amphiphilic properties which can be applied as surfactant, or ligands $;^{20}$ structure conformation which can be used as template in nanofabrication; ${ }^{21}$ compatibility 
with biomaterial in potential application; lower cost compared to the other polyelectrolytes there. $^{22}$

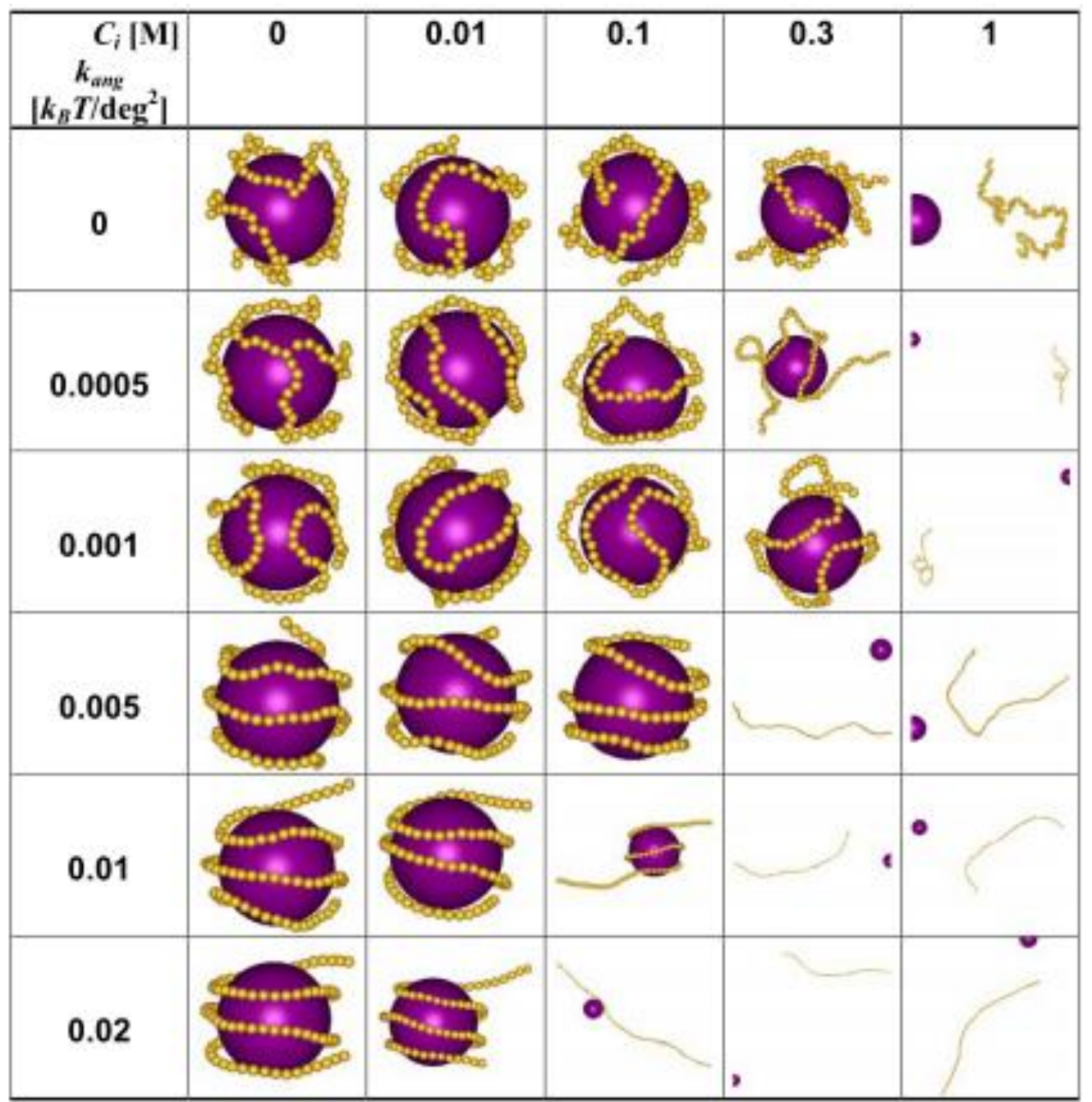

Figure 1.5. DNA precipitation diagram to show the relationship between DNA chain length, ionic strength, and its solubility. It is clear to see the solubility decrease while DNA chain length increase; the DNA solubility will decrease when the ionic strength increase. Reprinted with permission from ref 22 . Copyright 2008 from American Chemical Society.

Firstly, DNA or RDA precipitation study is based on the ionic properties. It is a long history for people to know the slat concentration control when they try to do 
the DNA, or RNA ionic separation. ${ }^{23}$ Just like polyelectrolyte DNA, or RNA solubility could be tune by adjusting solvent polarity. One method that people usually used to precipitate is called polarity (ethanol) precipitation. Secondly, DNA or RNA size effect on the properties has been studied very well. The chain size effects of DNA or RNA have been studied many years. ${ }^{24}$ According to figure 1.5 , it is clear to see the relationship between DNA chain length, ionic strength, and its solubility ${ }^{19}$. Thirdly, DNA, or RNA molecules were used during the nanostructure fabrication. ${ }^{21}$ Based on the charged chain it is easy to get highly ordered nanostructures when DNA or RNA molecules were applied as templated there. Most of the time, chiral nanostructures were synthesized there based on the template. ${ }^{25,27}$

In conclusion, polyelectrolytes are a kind of charged polymer which shows many different properties compared to other polymers. There are many parameters which could affect polyelectrolyte solubility, swelling, porosity, as well as morphologies. ${ }^{26}$ Polyelectrolytes have shown advantages when applied in nanomaterials, such as the amphiphilic domain, the highly ordered structure under control, and the counter ions interaction. ${ }^{14,27}$ All these advantages make polyelectrolytes a hot material in the world. ${ }^{28}$

\subsection{Hollowing behavior.}

Hollowing behavior is important to nanomaterials application, because it could improve the surface area, the porosity, and volume of nanomaterials. ${ }^{29}$ People 
studied hollowing behavior of nanomaterials in details to control it better. Hollow nanostructure shows high potential advantages in application, such as catalysts, battery, as well as solar cell because of its large surface area, large volume. ${ }^{30}$ Especially the hollow oxide nanomaterials, such as silica, $\mathrm{Ti}_{2} \mathrm{O}, \mathrm{Cu}_{2} \mathrm{O}, \mathrm{ZnO}$ have been used in many different areas for their unique properties there. ${ }^{31}$ More and more studies are focused on the hollowing behavior of oxide nanostructures in order to synthesize efficient material for application.

There are normally 3 main mechanisms for oxides hollowing behavior, surface selective etching under protection, ${ }^{32}$ Ostwald ripening, ${ }^{33}$ and surface redeposition. $^{34,35}$ 


\subsubsection{Selective etching.}
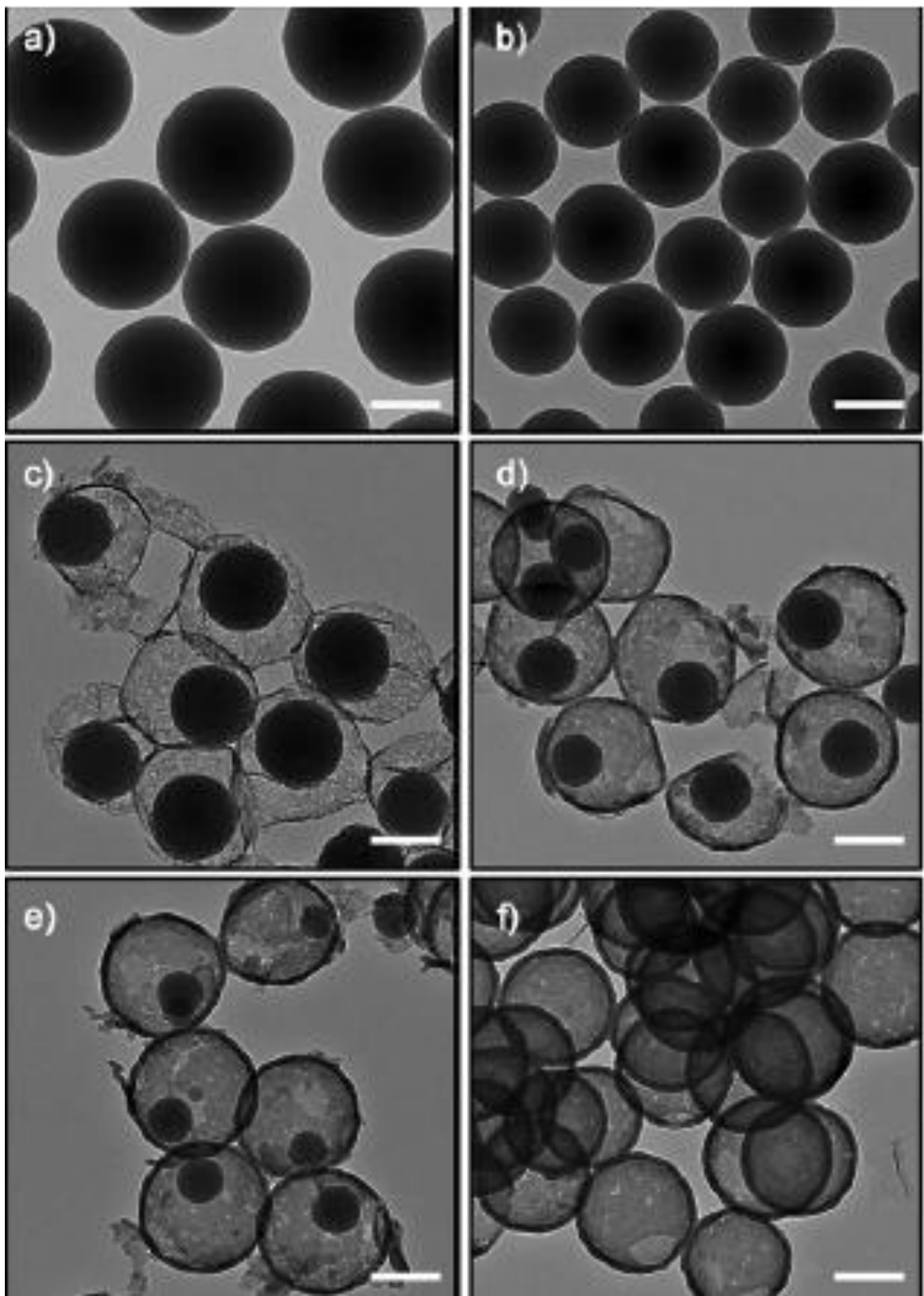

Figure 1.6. TEM images show the silica nanoparticles selective etching because

surfactant (PVP) protects on the outmost layer. (a) silica nanoparticle, (b) PVP

adsorbed silica nanoparticle, (c) PVP stabilized silica etching $30 \mathrm{~min}$, (d) $40 \mathrm{~min}$, 
(e) $50 \mathrm{~min}$, (f) $60 \mathrm{~min}$. Reprinted with permission from ref 33. Copyright 2008 from American Chemical Society.

Etching the unstable partial composition inside nanostructures, this including etching to remove the hard template, soft template, or the unstable facet caused by high surface energy. ${ }^{36}$ The etching mechanism is depends on the different methods during synthesis, including solubility difference, dissolving by chemical reaction, and so on. ${ }^{37}$

There are many examples about the surface selective etching to form hollow nanostructures there, such as silica nanoparticle. Just shown in figure 1.6, Professor Yin's group has reported that PVP can protect the outmost layer silica from etching while PVP absorbed on the silica nanoparticle surface. ${ }^{32}$ It cannot protect the inner layer because PVP molecule size is larger than silica pore size to diffuse inside. According to the TEM image in figure1.5 we can find that the etching happened from the interlayer of silica domain, not from the inner layer. With time going on, there is core-shell yolk shell structure after etching, then totally hollow silica nanoparticle was final product.

Besides the PVP used as protecting ligand during silica etching mentioned above, Yin group reported a cationic surfactant-assisted selective etching mechanism to get hollow silica nanoparticles. ${ }^{33}$ The mechanism is: solid silica dissolve first in base solution giving negatively charged species, and then CTAB cations can assist the redeposition of these negatively charged species, position 
of the soluble silicate species onto $\mathrm{sSiO}_{2}$. Such a silicate redeposition process consumes some released silicate species and thus kinetically drives the etching of sSiO2. The redeposition of the etched-out silicate allows more adsorption of CTA cations and eventually leads to the formation of the mesostructured $\mathrm{CTAB} / \mathrm{SiO}_{2}$ shell on the surface of $\mathrm{sSiO}_{2}$.

\subsubsection{Ostwald ripening.}

Ostwald ripening is a physical phenomenon that has been well known for more than a century. According to the IUPAC's terminology this process refers to "the growth of larger crystals from those of smaller size which have a higher solubility than the larger ones. ${ }^{36}$ For colloidal particles formed in solution media, there are many chemical equilibriums existing between the solid and liquid interfaces under each experimental setting. Because of non-uniformity of crystallites, concentrations of solutes (or growth nutrients) across the bulk solution vary. The homogenization of these concentration gradients will lead to complete dissolution of smaller crystallites as a result of the growth of the large crystallites. As this process involves matter relocation, one may use it as a new means for generation of interior space for the crystallite aggregates. In this connection, it is conceivable that the cavity will be generated in a location where crystallites are smaller and/or less compact when the Ostwald ripening proceeds. Likewise, more complex interior spaces can also be attained if the size distribution and aggregative patterns of as-formed primary crystallites are tiered. 
In most cases, however, large crystallites can be considered to be essentially immobile while the smaller ones are undergoing mass relocation through a solid-solution-solid process.

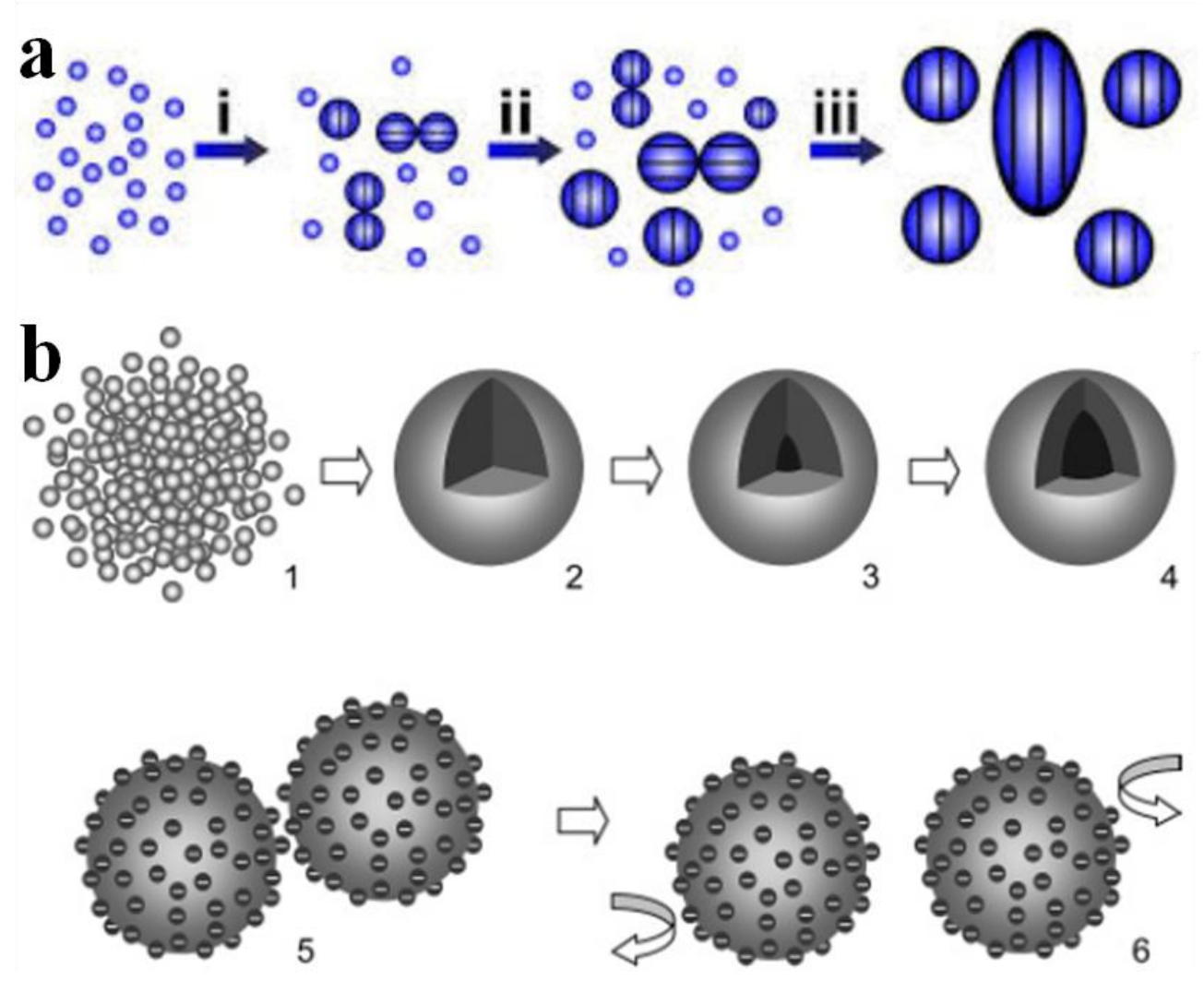

Figure 1.7. (a) Ostwald ripening mechanism: the smaller nanoparticles will dissolve and then redeposit on larger nanoparticle surface, which is caused by surface energy difference, (b) hollow nanoparticles formation based on Ostwald ripening mechanism: the nanoparticle would hollow from the inside to outside layer during recrystalization or annealing. Reprinted with permission from ref 36 . Copyright 2001 from American Chemical Society.

This thermodynamically-driven spontaneous process occurs because larger particles are more energetically favored than smaller particles. This stems from 
the fact that molecules on the surface of a particle are energetically less stable than the ones in the interior. ${ }^{38}$ As shown in figure 1.7, in colloidal the nucleation happens while the material concentration is over the saturated concentration value to give nucleus. The initial size fluctuation leads to the size difference among the nanoparticles showing broad size distribution. However, with the incubation time increase the size distribution will decrease to be narrow because of the smaller one dissolution and redeposition on the larger ones surface. For the core-shell nanoparticles with different material, or different level of crystallization, the Ostwald ripening process happens at the interface of the core-shell, which is caused by the lower stability of the internal layer just as shown in figure $1.7 \mathrm{~b}$. The morphology of the nanoparticles is not uniform, not smooth as shown in figure $1.7 \mathrm{c}$. the reason is that the redeposition process is also fluctuation, which means that the size, and shape of final nanoparticles are different even though the difference is not so obvious. Longer ripening time can give higher quality hollow nanoparticles by thermodynamic control. We will show many examples of hollowing structure preparation via Ostwald ripening mechanism in the following part.

There are many examples to show the hollowing behavior caused by Ostwald ripening, such as $\mathrm{TiO}_{2}$ nanoparticles, $\mathrm{SnO}$ nanoparticles, and so on. ${ }^{39,40}$

\subsubsection{Template sacrifice.}

Template method has been used to prepare hollow nanostructures for many 
years because of its unique advantages, such as easy control, simple procedure, high quality product, high reproducibility, as well as easy to scale up. The brief mechanism is as follow: using a hard/soft template to induce the second material deposition on the template while it is simple to give an exact morphology; then the template can be removed by chemical etching or calcinations to give hollow nanostructures. There are many different kinds of template to be used in the preparation of hollow nanostructures, such as lipid/surfactant micelles, metal/organic nanoparticles, carbon nanospheres/carbon nanotube, emulsion droplets, as well as bubbles.

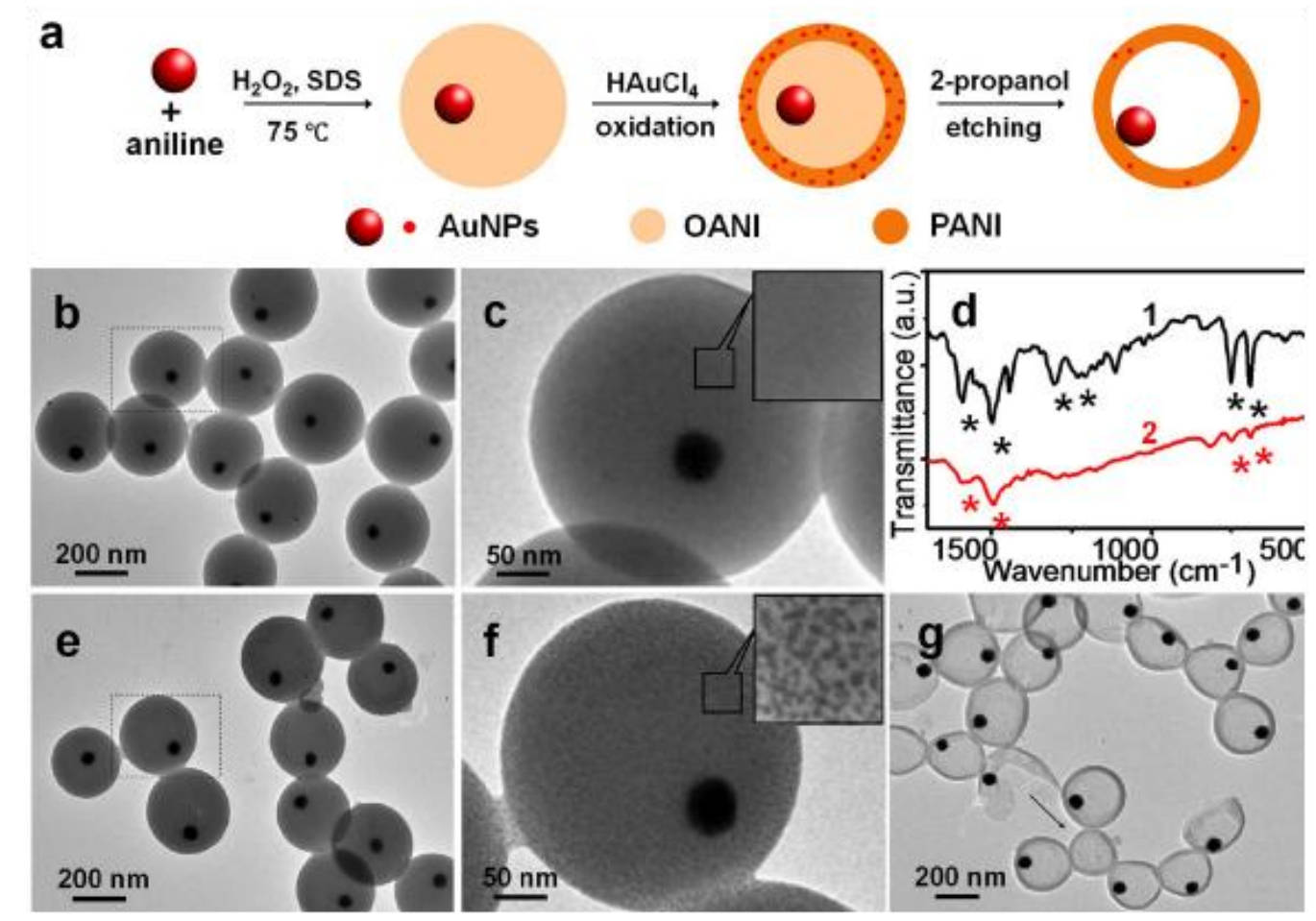

Figure 1.8. (a) scheme to show how to get hollow nanoparticle by template method: Au nanoparticle was coated by OANI to give core-shell structure, then the outmost layer of OANI was oxidized to PANI which is not soluble in IPA, and finally the OANI inner layer would be removed in organic solvent IPA while the 
outmost layer PANI was stable giving yolk shell hollow nanoparticles, (b) Au@OANI, (c) Au@OANI in high MAG, (d) UV spectra of core-shell nanoparticles, (e) Au@OANI@PANI, (f) Au@OANI@PANI in high MAG, (g) Au@PANI hollow nanoparticles. Reprinted with permission from ref 42. Copyright 2009 from American Chemical Society.

Normally people can use a template to synthesize different shape nanoparticles. ${ }^{41}$ Then try to remove the template by different methods, such as chemical etching, annealing, to get hollow nanostructures for application. ${ }^{42}$ The advantage for this method is that template can help to build a uniform shape which is critical to application, and then it is easy to remove the template. ${ }^{43}$ As shown in figure 1.9, the Au nanoparticle was coated by aniline to give core-shell nanoparticles, which will used as a hard template. Then the outmost layer of aniline will be oxidized to PANI which is insoluble in 2-isopropanol. Finally the aniline interlayer template can be removed by dispersing the nanoparticle into 2-isopropanol solution where the aniline is soluble but not the PANI.

Besides the above three mechanisms discussed in details, some other mechanisms have been applied to explain hollowing behavior, such as Kirkendall Effect, Galvanic Replacement, and so on. Normally, the Kirkendall effect refers to comparative diffusive migrations among different atomic species in metals and alloys under heating conditions. ${ }^{42}$ For example, zinc diffuses into copper faster than the copper diffuses into brass in a brass-copper interface due to their 
different atomic diffusivities. As a common result from this diffusion process, porosity will be generated in the lower-melting component side of the diffusion couple. Although void formation in alloys and solders may not be a desirable process for metallurgical manufacturing, the physical phenomenon may provide possibilities for fabrication of new nanomaterials with hollow interiors considering the directional matter flow and consequential vacancy accumulation in Kirkendall type diffusion. ${ }^{43}$ Galvanic replacement has been widely employed for general preparation of metal hollow nanostructures in a variety of shapes and sizes. ${ }^{37}$ In a typical reaction, the salt of a more noble metal (B) is reduced with preformed nanocrystals of a less noble metal (A), resulting in deposition of B on the surface of A. Upon complete consumption of metal A, hollow structures of metal B can be obtained under controlled conditions. The shape and cavity size of the derived hollow structure are then largely determined by the sacrificial nanocrystals of $\mathrm{A}^{45}$

To the above different hollowing behavior mechanisms we still have some questions that cannot answer by the mechanism itself, such as why does the hollowing start from the interlayer but not from the inner layer, or outmost layer? Why does the redeposition only happen on the outmost surface while there are many different kinds of interface such as, solvent-inner layer, void-inner layer, and so on? Why does ripening happen inside the core domain while the concentration is higher inside than it is outside the nanoparticle domain? 
We think the hollowing behavior mechanism should be studied in depth based on the pioneer study, which should be focused on the chemical difference analysis linked with thermodynamic and kinetic theory. It is necessary for us to design experiments after this review, especially to the study of silica hollowing behavior.

\subsection{Core-shell nanostructure.}

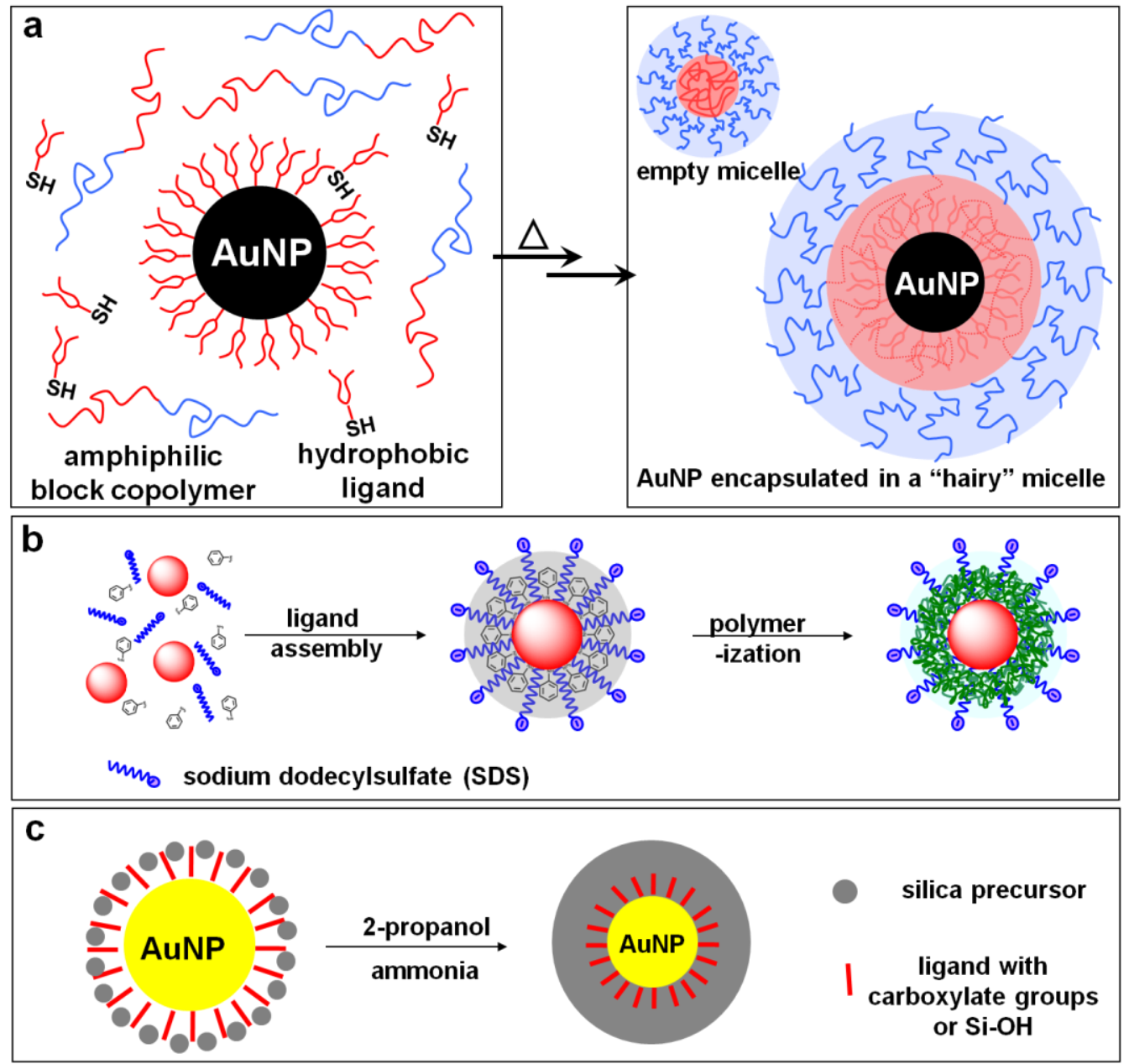

Figure 1.9. (a) Au nanoparticle surface modification to prepare core-shell nanoparticles, (b) surfactant induces micelle self-assemble to give core-shell nanoparticles, (c) nanoparticle surface heterogeneous nucleation to give 
core-shell nanoparticles. Reprinted with permission from ref 25, 41, 51, respectively. Copyright 2010 from American Chemical Society.

Over the past years, single domain nanoparticle has been studied very well deeply. Now, more and more people focus on the two or multi domains nanomaterials for high potential application. More than one domain nanomaterials show advantages on application, such as high compatibility with cells, high solubility in different kinds of solvents, high stability, high capacity, and so on. ${ }^{44,45}$ For such nanostructures, the additional interface between the domains must be considered. To the mechanism and synthesis study, one more domain introduces more interfaces among these materials which is important to its morphology and property. ${ }^{46}$

Here we just focus on the two domain nanoparticles. Actually, the double domain nanoparticles are formed after the surface "wetting" of one material over another one. $^{47}$

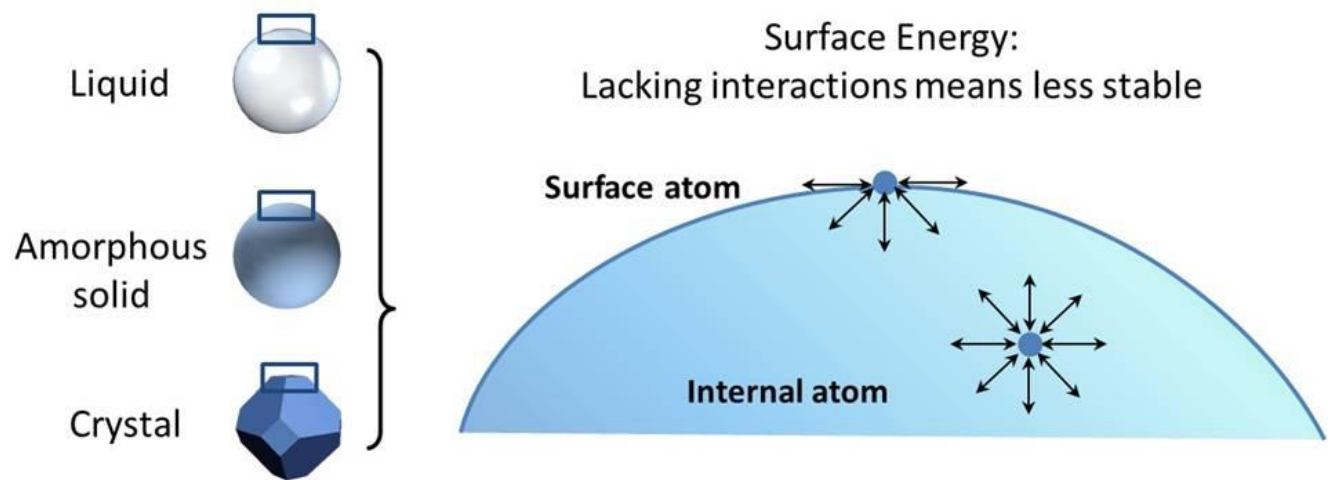

Figure 1.10. The essence of nanoparticle surface energy theory: The minimization of system energy required by themodynamic stability requires the 
minimization of surface atoms with unfavorable interactions, which means the S/V ratio minimium value. Reprinted with permission from ref 52. Copyright 2014 from American Chemical Society.

There are many methods to prepare core-shell double domain nanoparticles. These methods try to tune the equilibrium of homogeneous nucleation and heterogeneous nucleation, interfacial energy, surface tension, surface volume ratio, and so on. ${ }^{48}$ We will try to introduce nucleation theory, interfacial energy theory, and some popular methods to prepare double-domain nanoparticles in the following issues.

\subsubsection{Nucleation and surface deposition.}

Nucleation is the first stage of any crystallization and deposition process in the nanosynthesis although it is difficult to test it directly. The nuclei in the real pace are difficult to check for the lack of experimental tools to capture it by monitoring, and identifying. Normally there are two kinds of nucleation, homogeneous nucleation, and heterogeneous nucleation. Both the homogeneous nucleation and heterogeneous nucleation are the fast, first stage of the nanomaterials formation. $^{36}$

Homogeneous nucleation (as shown in figure 1.10) represents the growth of nanomaterials sharing the similar process during the nucleation and deposition: there is a critical concentration value after the chemical reaction for the 
homogeneous solution to give nuclei; there is a similar main kinetic barrier to form the nuclei. ${ }^{49}$ The primary nuclei have high surface energy because of extremely small size at the beginning of the nucleation. The kinetic pathway under which the nanoparticle growth depends on how this kinetic barrier being crossed in the homogeneous solution. Normally single domain nanoparticle growth in one step is a homogeneous nucleation and deposition process.

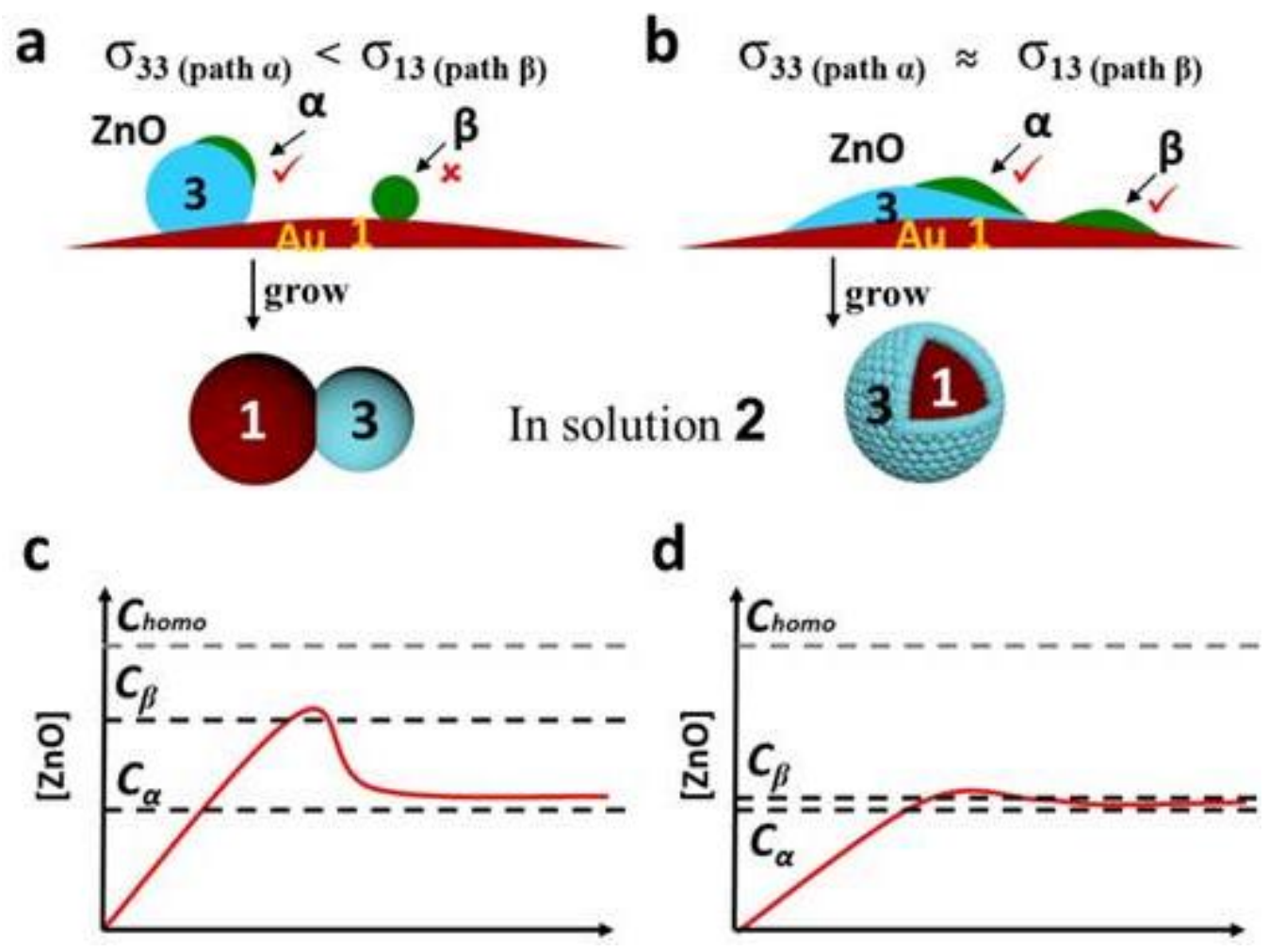

Figure 1.11. (a) if interfacial energy between nanoparticle and shell is larger than that between shell and solvent, or substrate, there will be partially coating shell, (b) if interfacial energy between nanoparticle and shell is smaller than that between shell and solvent, or similar, there will be full encapsulation, (c) in condition a, the homogeneous nucleation is favorable, (d) in condition $b$, the heterogeneous nucleation is favorable. Reprinted with permission from ref. 54, 
respectively. Copyright 2013 from American Chemical Society.

Heterogeneous nucleation is a different primary nucleation process with homogeneous nucleation from the energy barrier or the kinetic barrier during the nucleation and deposition process. ${ }^{50}$ To be exactly, the heterogeneous nucleation is normally describing the uniformly nucleation and deposition, which is caused by lower kinetic barrier compared to homogeneous nucleation. ${ }^{51}$ Heterogeneous nucleation is to a procedure that could keep the rate of production slower than that of consumption (heterogeneous nucleation on the existing nuclei), which suppresses a homogeneous nucleation, allows all nuclei to emerge and grow at roughly the same time, under the same rate. It is clear to see that heterogeneous nucleation is easier to control compared to homogeneous nucleation. ${ }^{52}$

The formation of core-shell nanoparticle is the process of nucleation and deposition control. When we dune the kinetic barrier during the nucleation and deposition procedure (as shown in figure 1.11) there will give different kinds of core-shell nanoparticles. The kinetic barrier tuning was conducted by different methods, such as surface ligand modification, phase modification, surfactant modification, as well as lattice mismatch, surface defect control. All these methods can change the interfacial energy (kinetic barrier) between the two different domain materials. ${ }^{53}$ The interfacial energy adjustment could affect the surface nucleation and deposition process, which is critical to the final morphologies of the core-shell nanomaterials. ${ }^{48}$ 


\subsubsection{Double-domain nanoparticle morphology.}

With multiple components and multiple processes in a system, it is conceivable that its energy landscape is significantly more complex than that of the single-component systems. In the deposition of shell materials on core NPs, for example, one need to consider the coordination and dissociation of ligands on the cores, the nucleation process and the second growth of the shell materials, the core-shell configuration or morphologies, as well as the ligand coordination to the shells. Given the different processes and their possible interplay, mixed T-K arguments are rather common in such systems. ${ }^{48}$

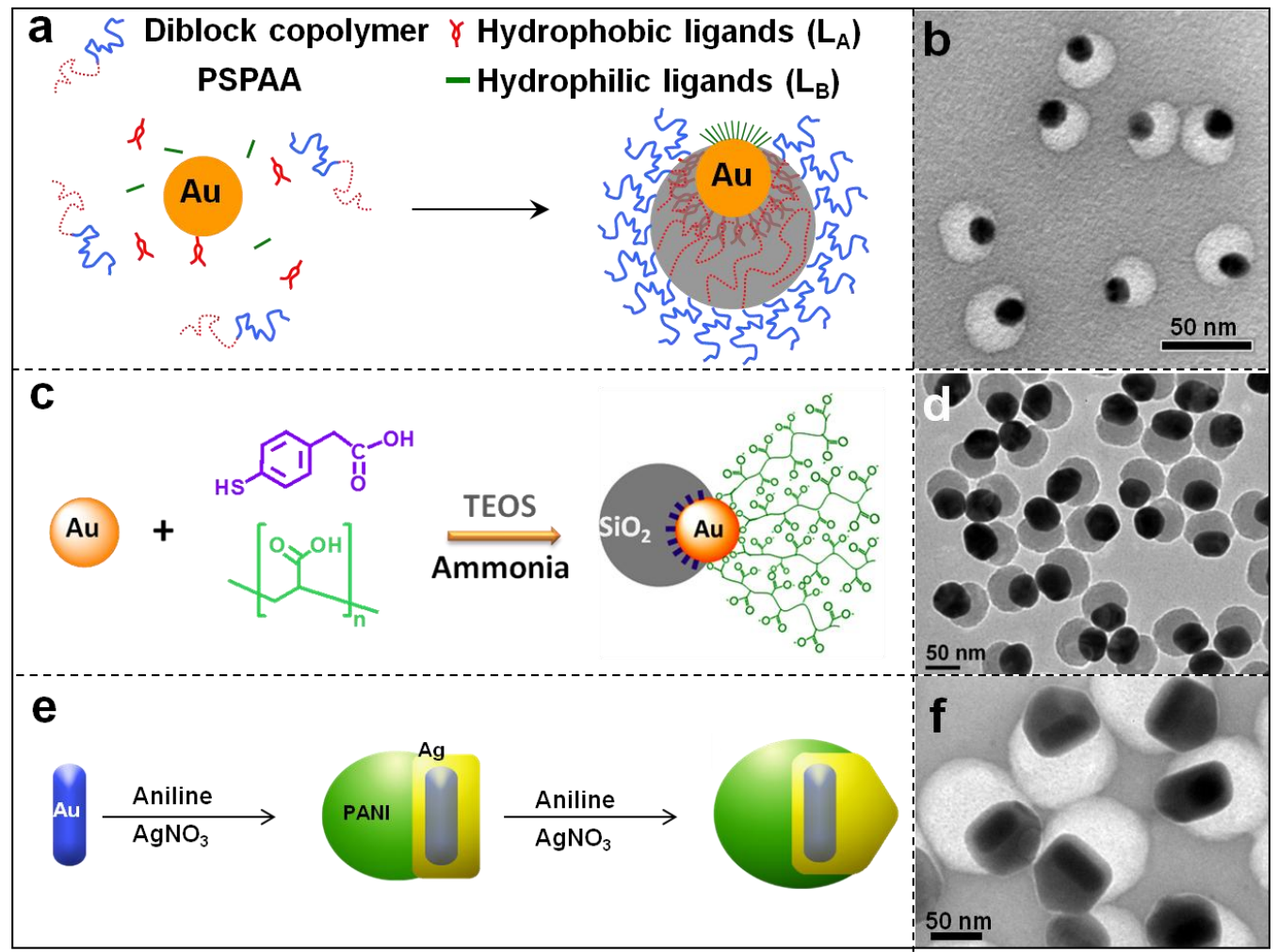

Figure 1.12. Double domain hybrid nanostructure morphologies: (a, b) surface ligand patterning control to give core-shell or Janus Au@ polymer nanoparticles, 
(c, d) surface ligand blocking control to give Au@silica core-shell or Janus nanoparticles, (e, f) facet control to give Au-Ag@polymer core-shell or Janus nanoparticles. Reprinted with permission from ref 37, 56, 60, respectively. Copyright 2010-2012 from American Chemical Society.

The main factor to the heterostructure morphology is the energy balance or interfacial energy between the metal core and the shells. There are many different kinds of heterostructures there, such as metal-metal, organic-organic heterostructure, organic-inorganic nanostructure, as well as other kinds. ${ }^{54}$ The morphologies are also various, such as concentric, eccentric, Janus, or branch nanomaterials. In this chapter we focused on the core-shell double-domain nanostructure to study their morphologies formation mechanism.

\subsubsection{Metal NP@oxide core-shell nanoparticle.}

Metal nanoparticles coated by oxide shell have been studied recently, and become a hot topic. The oxide shells are popular for many reasons: simple method to prepare, lower cost, broad resources, and so on. Besides, oxide shells have special excellent properties to application, such as bio compatibility $\left(\mathrm{Si}_{2} \mathrm{O}\right)$, photo conductivity $\left(\mathrm{TiO}_{2}\right)$, and photon conductivity $\left(\mathrm{Cu}_{2} \mathrm{O}\right) .{ }^{55}$ Those properties make them popular in application such as biosensor, drug delivery, battery, and catalysts for chemical reaction. $^{45,46}$

In order to get core-shell metal NP@oxide nanoparticles people try to decrease 
the interfacial energy between the metal core and oxide shell to induce the heterogeneous nucleation. There are many different methods to lower the interfacial energy between metal core and polymer shell: surface modification, ${ }^{53}$ directly surface heterogeneous nucleation, ${ }^{54}$ shell formation under core oxidation control following the void coalescence in the system. We will describe the different methods in the following paragraphs.

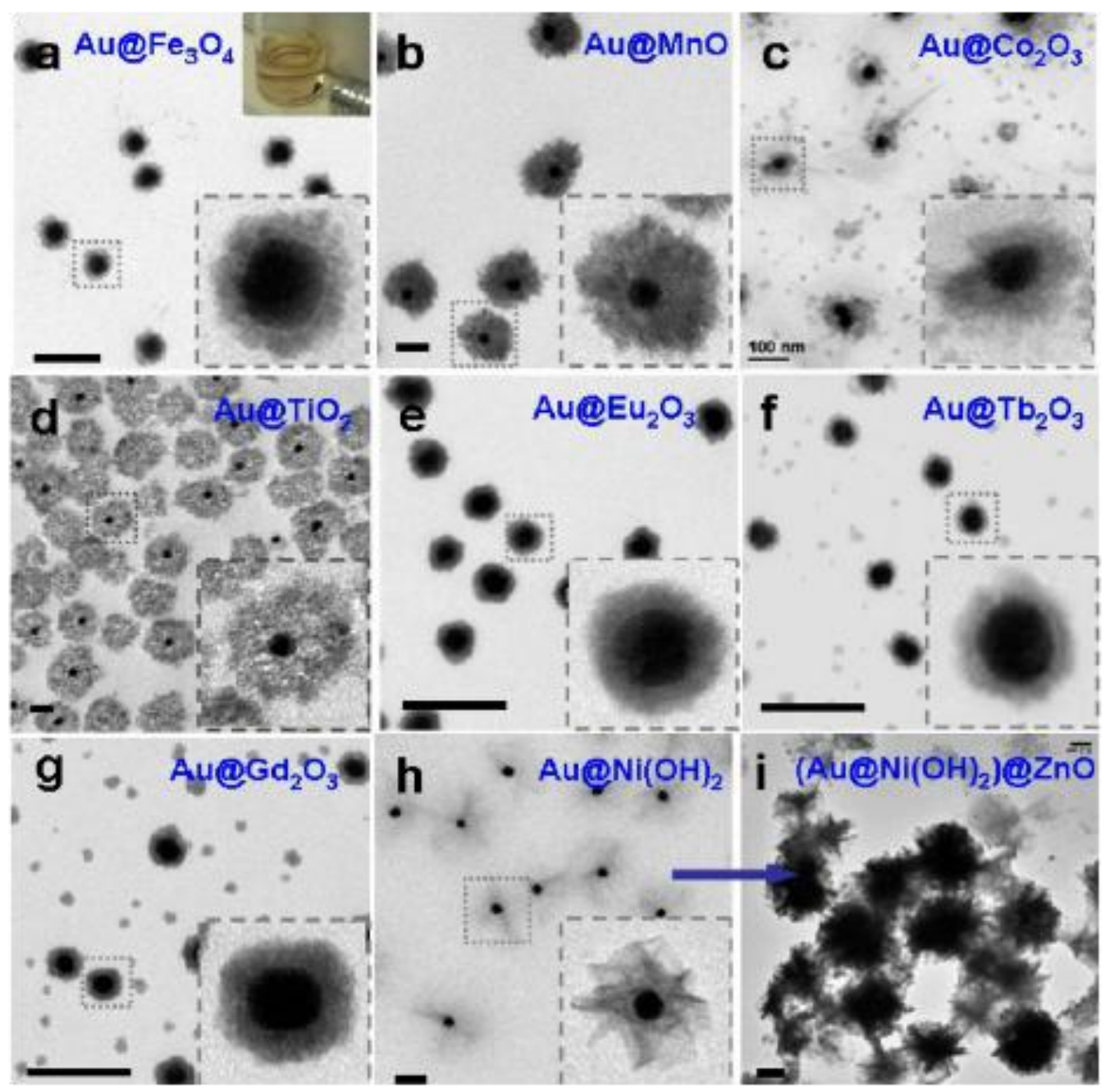

Figure 1.13.TEM images showing different kinds of oxide shells encapsulation of Au nanoparticles after the ligand surface modification, or surfactant assembly: (a) $\mathrm{Au} @ \mathrm{Fe}_{3} \mathrm{O}_{4}$ core-shell nanoparticle, (b) $\mathrm{Au} @ \mathrm{MnO}$ core-shell nanoparticle, (c) 
$\mathrm{Au} @ \mathrm{Co}_{2} \mathrm{O}_{3}$ core-shell nanoparticle, (d) $\mathrm{Au} @ \mathrm{TiO}_{2}$ core-shell nanoparticle, (e) $\mathrm{Au} @ \mathrm{Eu}_{2} \mathrm{O}_{3}$ core-shell nanoparticle, (f) $\mathrm{Au} @ \mathrm{~Tb}_{2} \mathrm{O}_{3}$ core-shell nanoparticle, (g) $\mathrm{Au} @ \mathrm{Gd}_{2} \mathrm{O}_{3}$ core-shell nanoparticle, (h) $\mathrm{Au} @ \mathrm{Ni}(\mathrm{OH})_{2}$ core-shell nanoparticle, and (i) $\left(\mathrm{Au} @ \mathrm{Ni}(\mathrm{OH})_{2}\right) @ \mathrm{ZnO}$ core-shell nanoparticle. All the core-shell structure is uniform, high productivity. Scale bar: $200 \mathrm{~nm}$. Reprinted with permission from ref 63, respectively. Copyright 2013 from American Chemical Society.

Direct heterogeneous nucleation on the core seeds is conducted under mild temperature which could perform a slow addition of second material by control the reactivity of the system. ${ }^{56}$ Normally metal seeds are dispersed into organic/water mixture in the presence of surfactant which is used to stabilize the nanoparticles, as well as the aqueous phase. ${ }^{46}$ Then the shell precursor (transition metal-alkoxide precursor was normally used) will hydrolyze followed by condensation to give a uniform oxide shell. ${ }^{57}$

Surface ligand modification (figure 1.11) is another way to decrease the interfacial energy between metal core and oxide shell. ${ }^{53}$ The ligands molecules have been applied into the coating process for the reason that it can lower the interfacial energy between nanocrystal seeds and oxide shells, which could be possible to excess the energy barrier for the heterogeneous nucleation. ${ }^{48}$ There are many examples for the surface modification control during the oxide shell coating nanocrystals. Different kinds of double-domain nanoparticle can be collected, such as concentric core-shell nanoparticle, eccentric core-shell 
nanoparticle, as well as Janus nanoparticle there.

The conversion of core to shell under redox replacement reaction (figure 1.12) is an effective way to prepare metal NP@oxide core-shell nanoparticles. ${ }^{54}$ Normally this method is applied to synthesize $\mathrm{M} @ \mathrm{M}_{\mathrm{x}} \mathrm{O}_{\mathrm{y}}$ core-shell nanoparticles. In this system, the transition metal nanocrystals are the favor materials because they show many advantages there: being a kind of substrate; easily being oxidized; easy to be investigated after oxidized. ${ }^{58}$ For example, Co nanocrystals show high reactivity to be oxidized while exposed to the air, which means a high susceptibility. ${ }^{59}$ There will be a polycrystalline layer of $\mathrm{CoO}$ on the surface of $\mathrm{Co}$ core which is easily to be detected.

\subsubsection{Metal NP@ polymer core-shell nanoparticle.}

Recently, besides the oxide shell, more and more polymer core-shell nanoparticles have been synthesized for the higher stability, structure diversity, and so on. ${ }^{53}$ Here we organized the literatures about metal nanoparticles coated by polymer shell, which have been applied to hydrophobic nanoparticles, metal-polymer crystals, and so on. ${ }^{60}$

Previously, the PSPAA encapsulation of AuNPs, $\gamma-\mathrm{Fe}_{2} \mathrm{O}_{3}$, quantum dots (QDs), and carbon nanotubes (CNTs) have been reported, where the slow addition of water to solubilized PSPAA in an organic solvent induced the polymer self-assembly as shown in figure 1.14 . We also reported a modified approach to induce PSPAA self-assembly by heating NPs with PSPAA in mixture solvents. 
AuNPs, AuNWs, and CNT bundles were encapsulated. The main method to prepare metal-polymer core-shell nanoparticles is based on phase separation methodology. ${ }^{61}$ According to literatures there are 3 main mechanisms for phase separation method: polymer swelling volume fraction among monomers and interactions among them is critical to demined the interaction forces among monomers and polymers,

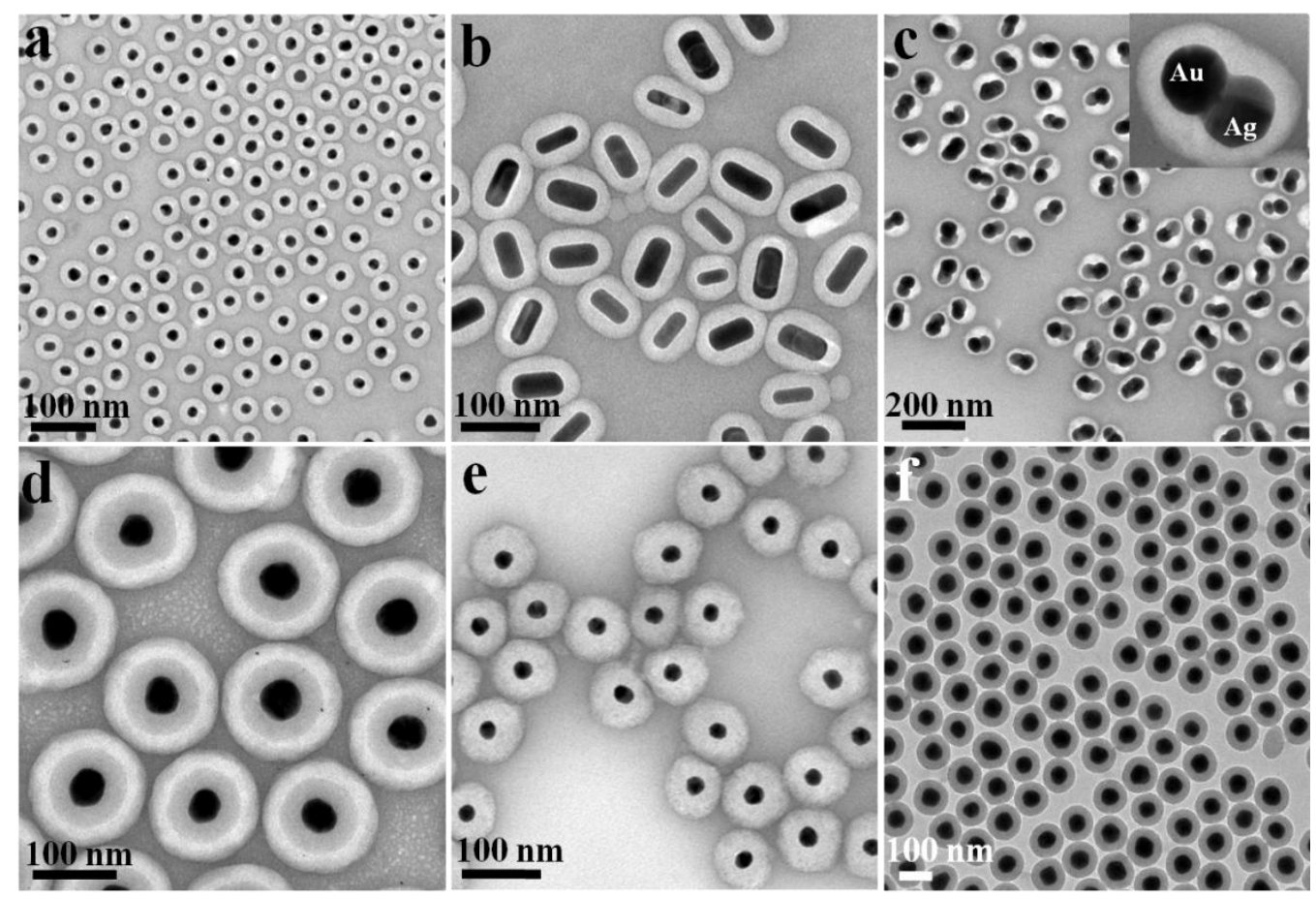

Figure 1.14. TEM images of metal nanocrystals coated by polymer shell: (a) AuNP@PSPAA core-shell nanoparticle; (b) Au NR@PSPAA core-shell nanoparticle; (c) (Au-Ag dimer)@PSPAA core-shell nanoparticle; (d) doublelayer (Au@ $\left.\mathrm{SiO}_{2}\right) @$ PSPAA core-shell nanoparticle; (e) AuNP@PANI core-shell nanoparticle; (f) AuNP@ $\mathrm{SiO}_{2}$ core-shell nanoparticle as compared data. ${ }^{61,62}$ Reprinted with permission from ref. 60, 61, respectively. Copyright 2011 and 2012 from American Chemical Society. 
oligomers there; the interfacial energy between metal nanoparticle and solvent, polymer; the solubility of polymer, monomers. The advantage is that phase separation method is easy, diversity, which means that phase separation is difficult to control final morphology of nanoparticles there. Besides phase separation (emulsion, reverse emulsion), templating, surface modification, lithography, as well as electrohydrodynamic jetting techniques have been applied to prepare different metal polymer core-shell nanoparticles. ${ }^{54}$

For the phase separation method, there are many reports to show the procedure by tuning different parameters. Such as Dr. Yang group proposed a way of preparing polymer Janus nanoparticles at emulsion interface, which was ion-water emulsion heated at $70{ }^{\circ} \mathrm{C}$ to initiate the polymerization during the polymer nanoparticle formation because of phase reparation process. ${ }^{63}$ Injecting method is another example to get the polymer nanoparticles formation. Under this method people design a special kind of Y type tube being used as a channel to introduce two opposite kinds of solvent stream inside the main chamber: one is monomer flow which is the main precursor for reaction, while another flow is the droplet of opposite solubility with monomer flow. ${ }^{64}$ Finally the phase separation might happen during the reaction in the main chamber, a kind of nanoreactor. Normally, the polymerization reaction was induced by many different light sources to initiate polymerization, such as UV light, laser, sonication, and so on. $^{64}$ 
By adjusting the interfacial energy there people can tune the shell morphology of metal-polymer core-shell nanoparticles. ${ }^{65}$ Ligands, temperature, solvent ratio, as well as monomer concentration are critical to the final morphologies of polymer shells. ${ }^{48}$ A lot of examples have been reported during the past decades on the polymer morphologies control, which might include Dr. Xing from professor Chen's group used $\mathrm{Au}$ nanoparticles as seeds encapsulated by polyaniline, while the Au seed was stabilized by sodium citrate as surface ligand. ${ }^{66}$ In order to control polymer shell overgrowth on seed surface there are many different methods applied to control, such as ligand partially blocking, Ag selective surface deposition, SDS or PVP combined with ligand blocking to control the surface nucleation and deposition process, and so on. ${ }^{67}$

In essence, templated growth of nanocrystals can be viewed as the growth of the crystalline domain on the surface of the template, making it a bi-component system. ${ }^{68,69}$ Should the template-nanocrystal interfacial energy be high, the formation of the interface would be both kinetically and thermodynamically unfavorable, leading to homogeneous nucleation of free nanocrystals in the solution unaffected by the template. Thus, for all known cases of templated growth, the interfacial energy must be low enough to allow conformal coating We note that the colloidal growth of ultrathin NWs, ${ }^{70,71}$ often proposed to be the result of templated growth, is very different from the growth of most other nanocrystals. The scope of the applicable templates is extremely narrow. For 
example, the width of the ultrathin NWs is unusually monodispersed and rarely tunable. $^{70,72}$ What stops their lateral growth when a certain width is reached? Growing long NWs from short NW has not been demonstrated. The NWs have not been shown to grow from a bulk crystal surface. ${ }^{73}$ More insights in the templated growth may help elucidate these mysteries and improve the synthetic design.

\subsection{Summary and outlook.}

In the formation of nanostructures, the microscopic processes are random and dynamic. Among the chaos, orderly nanostructures are known to emerge, including their regular shape, size uniformity, and extensive superstructures. ${ }^{74}$ Such ordering is the basis of nanoscience and nanotechnology; ${ }^{55}$ and understanding its origin is of pivotal importance. We must start from the fundamental principles in growing and assembling the basic building blocks, before sophisticated nanostructures can be made for advanced applications. To establish the mechanistic proposals on the basis of energy terms is a first step towards rational design and systems approach.

Using simple geometric analysis and logical deduction, it is indeed possible to compare the relative stability of nanostructures during their growth, assembly and shape evolution. The detailed processes therein were articulated and carefully analyzed, so that the mechanistic proposals were unambiguously presented. The fundamental differences between the thermodynamically and 
kinetically controlled scenarios can be a useful tool in mechanistic proposals and novel structure design in future. 


\subsection{Bibliography}

(1) Raspaud, E.; De La Cruz, M. O.; Sikorav, J.-L.; Livolant, F. Biophys. J. 1998, 74,381 .

(2) Miller, S.; Dykes, D.; Polesky, H. Nucleic Acids Res. 1988, 16, 1215.

(3) Sukhorukov, G.; Dähne, L.; Hartmann, J.; Donath, E.; Möhwald, H. Advanced Materials 2000, 12, 112.

(4) Langevin, D. Adv. Colloid Interface Sci. 2009, 147, 170.

(5) Hoover, M. F. Journal of Macromolecular Science-Chemistry 1970, 4, 1327.

(6) Wittmer, J.; Johner, A.; Joanny, J. Journal de Physique II 1995, 5, 635.

(7) Wang, D.; Gong, X.; Heeger, P. S.; Rininsland, F.; Bazan, G. C.; Heeger, A. J. Proceedings of the National Academy of Sciences 2002, 99, 49.

(8) Yang, M.; Yang, Y.; Yang, H.; Shen, G.; Yu, R. Biomaterials 2006, 27, 246.

(9) Antonietti, M.; Conrad, J.; Thuenemann, A. Macromolecules 1994, 27, 6007.

(10) Gueron, M.; Weisbuch, G. Biopolymers 1980, 19, 353.

(11) Muthukumar, M. The Journal of chemical physics 1987, 86, 7230.

(12) Tong, W.; Song, H.; Gao, C.; Möhwald, H. The Journal of Physical Chemistry B 2006, 110, 12905.

(13) Büscher, K.; Graf, K.; Ahrens, H.; Helm, C. A. Langmuir : the ACS journal of surfaces and colloids 2002, 18, 3585 .

(14) Lankalapalli, S.; Kolapalli, V. Indian journal of pharmaceutical sciences 2009, 71,481 . 
(15) Viovy, J.-L. Reviews of Modern Physics 2000, 72, 813.

(16) Chen, H.; Meisburger, S. P.; Pabit, S. A.; Sutton, J. L.; Webb, W. W.; Pollack, L. Proceedings of the National Academy of Sciences 2012, 109, 799.

(17) Guo, P. Journal of nanoscience and nanotechnology 2005, 5, 1964.

(18) Heller, M. J. Annual review of biomedical engineering 2002, 4, 129.

(19) Xia, F.; Zuo, X.; Yang, R.; Xiao, Y.; Kang, D.; Vallée-Bélisle, A.; Gong, X.; Yuen, J. D.; Hsu, B. B.; Heeger, A. J. Proceedings of the National Academy of Sciences 2010, 107, 10837.

(20) Tuerk, C.; Gold, L. Science 1990, 249, 505.

(21) Becerril, H. A.; Woolley, A. T. Chemical Society Reviews 2009, 38, 329.

(22) Mertig, M.; Colombi Ciacchi, L.; Seidel, R.; Pompe, W.; De Vita, A. Nano Letters 2002, 2, 841.

(23) Lee, G.-B.; Chen, S.-H.; Huang, G.-R.; Sung, W.-C.; Lin, Y.-H. Sensors and Actuators B: Chemical 2001, 75, 142.

(24) Quake, S. R.; Scherer, A. Science 2000, 290, 1536.

(25) Pilo-Pais, M.; Goldberg, S.; Samano, E.; LaBean, T.; Finkelstein, G. Nano letters 2011, 11, 3489.

(26) Berg, M. C.; Zhai, L.; Cohen, R. E.; Rubner, M. F. Biomacromolecules 2006, 7, 357.

(27) Zhai, L.; Cebeci, F. C.; Cohen, R. E.; Rubner, M. F. Nano letters 2004, 4, 1349. 
(28) Gao, C.; Leporatti, S.; Moya, S.; Donath, E.; Möhwald, H. Langmuir : the ACS journal of surfaces and colloids 2001, 17, 3491.

(29) Lou, X. W. D.; Archer, L. A.; Yang, Z. Advanced Materials 2008, 20, 3987.

(30) Zhang, Q.; Wang, W.; Goebl, J.; Yin, Y. Nano Today 2009, 4, 494.

(31) Yang, H. G.; Zeng, H. C. The Journal of Physical Chemistry B 2004, 108, 3492.

(32) Zhang, T.; Ge, J.; Hu, Y.; Zhang, Q.; Aloni, S.; Yin, Y. Angewandte Chemie 2008, $120,5890$.

(33) Yec, C. C.; Zeng, H. C. Journal of Materials Chemistry A 2014, 2, 4843.

(34) Oh, W.-K.; Kim, S.; Choi, M.; Kim, C.; Jeong, Y. S.; Cho, B.-R.; Hahn, J.-S.; Jang, J. ACS nano 2010, 4, 5301.

(35) Lou, X. W.; Wang, Y.; Yuan, C.; Lee, J. Y.; Archer, L. A. Advanced Materials 2006, 18, 2325.

(36) Turnbull, D.; Fisher, J. C. The Journal of chemical physics 1949, 17, 71.

(37) Zeng, H.; Cai, W.; Liu, P.; Xu, X.; Zhou, H.; Klingshirn, C.; Kalt, H. ACS nano 2008, 2, 1661.

(38) Voorhees, P. W. Journal of Statistical Physics 1985, 38, 231.

(39) Li, J.; Zeng, H. C. J. Am. Chem. Soc. 2007, 129, 15839.

(40) Lou, X. W.; Yuan, C.; Rhoades, E.; Zhang, Q.; Archer, L. A. Advanced Functional Materials 2006, 16, 1679. 
(41) Huang, J.; Xie, Y.; Li, B.; Liu, Y.; Qian, Y.; Zhang, S. Advanced Materials 2000, 12,808 .

(42) Sun, Y.; Mayers, B. T.; Xia, Y. Nano Letters 2002, 2, 481.

(43) Zhong, Z.; Yin, Y.; Gates, B.; Xia, Y. Advanced Materials 2000, 12, 206.

(44) Xiong, H.-M.; Xu, Y.; Ren, Q.-G.; Xia, Y.-Y. J. Am. Chem. Soc. 2008, 130, 7522

(45) Zhong, C.-J.; Maye, M. M. Advanced Materials 2001, 13, 1507.

(46) Ghosh Chaudhuri, R.; Paria, S. Chem. Rev. 2011, 112, 2373.

(47) Yuan, Y.; Lee, T. R. In Surface science techniques; Springer: 2013, p 3.

(48) Wang, Y.; He, J.; Liu, C.; Chong, W. H.; Chen, H. Angewandte Chemie International Edition 2015, 54, 2022.

(49) Abraham, F. Homogeneous nucleation theory: the pretransition theory of vapor condensation; Elsevier, 2012; Vol. 1.

(50) Turnbull, D. The Journal of Chemical Physics 1950, 18, 198.

(51) Fievet, F.; Lagier, J.; Blin, B.; Beaudoin, B.; Figlarz, M. Solid State Ionics 1989, 32, 198.

(52) Sun, H.; He, J.; Wang, J.; Zhang, S.-Y.; Liu, C.; Sritharan, T.; Mhaisalkar, S.; Han, M.-Y.; Wang, D.; Chen, H. J. Am. Chem. Soc. 2013, 135, 9099.

(53) Wang, H.; Chen, L.; Feng, Y.; Chen, H. Accounts of Chemical Research 2013, 46, 1636.

(54) Carbone, L.; Cozzoli, P. D. Nano Today 2010, 5, 449. 
(55) Qi, J.; Dang, X.; Hammond, P. T.; Belcher, A. M. ACS nano 2011, 5, 7108.

(56) Pan, M.; Sun, H.; Lim, J. W.; Bakaul, S. R.; Zeng, Y.; Xing, S.; Wu, T.; Yan, Q.; Chen, H. Chemical communications 2012, 48, 1440.

(57) Guerrero-Martínez, A.; Pérez-Juste, J.; Liz-Marzán, L. M. Advanced Materials 2010, 22, n/a.

(58) Nogués, J.; Skumryev, V.; Sort, J.; Stoyanov, S.; Givord, D. Physical review letters 2006, 97, 157203.

(59) Wiedwald, U.; Fauth, K.; Heßler, M.; Boyen, H. G.; Weigl, F.; Hilgendorff, M.; Giersig, M.; Schütz, G.; Ziemann, P.; Farle, M. ChemPhysChem 2005, 6, 2522. (60) Liu, C.; Chen, G.; Sun, H.; Xu, J.; Feng, Y.; Zhang, Z.; Wu, T.; Chen, H. small 2011, 7, 2721.

(61) Yao, L.; Liu, C.; Chong, W. H.; Wang, H.; Chen, L.; Chen, H. Small 2015, $11,232$.

(62) Liu, C.; Yao, L.; Wang, H.; Phua, Z. R.; Song, X.; Chen, H. Small 2014, 10, 1332.

(63) Song, L.; Qiao, Y.; Liu, Z.; Li, Y. Polymer Chemistry 2015, 6, 896.

(64) Hu, J.; Zhou, S.; Sun, Y.; Fang, X.; Wu, L. Chemical Society Reviews 2012, $41,4356$.

(65) Zhu, L.; Wang, H.; Shen, X.; Chen, L.; Wang, Y.; Chen, H. Small 2012, 8, 1857.

(66) Sun, H.; Shen, X.; Yao, L.; Xing, S.; Wang, H.; Feng, Y.; Chen, H. J. Am. 
Chem. Soc. 2012, 134, 11243.

(67) Song, J. E.; Phenrat, T.; Marinakos, S.; Xiao, Y.; Liu, J.; Wiesner, M. R.; Tilton, R. D.; Lowry, G. V. Environmental science \& technology 2011, 45, 5988.

(68) Jones, M. R.; Osberg, K. D.; Macfarlane, R. J.; Langille, M. R.; Mirkin, C. A. Chem. Rev. 2011, 111, 3736.

(69) Martin, C. R. Chem. Mater. 1996, 8, 1739.

(70) Huo, Z.; Tsung, C.-k.; Huang, W.; Zhang, X.; Yang, P. Nano Lett. 2008, 8, 2041.

(71) Wang, N.; Cai, Y.; Zhang, R. Q. Mater. Sci. Eng., R 2008, 60, 1.

(72) Wang, C.; Hu, Y.; Lieber, C. M.; Sun, S. J. Am. Chem. Soc. 2008, 130, 8902.

(73) Xu, J.; Wang, H.; Liu, C.; Yang, Y.; Chen, T.; Wang, Y.; Wang, F.; Liu, X.; Xing, B.; Chen, H. J. Am. Chem. Soc. 2010, 132, 11920.

(74) Ding, Y.; Gao, P. X.; Wang, Z. L. J. Am. Chem. Soc. 2004, 126, 2066. 


\section{Chapter 2 Synthesis and Etching Study of Stöber Silica Nanoparticles}

\subsection{Introduction}

Silica is a hot material for its broad application in many different areas. Here we just summarized its application by surface coating of different nanomaterials.

Silica coated nanomaterials to give core-shell nanoparticles are gradually attracting more and more attention with modified properties. Silica core-shell structures have advantages as follows: firstly, it reduces the bulk conductivity and increases the suspension stability of the core particles. Secondly, because of the most chemically inertness of silica, it can block the core surface without interfering in the redox reaction at the core surface. Thirdly, it can also modulate the position and intensity of the surface Plasmon absorbance band since silica is optically transparent. ${ }^{1}$ Hence, researchers have concentrated more on silica coatings on different inorganic core materials such as metals, binary inorganic composites, metal oxides, and metalsalts. ${ }^{1-2}$ Especially, the metal cores include $\mathrm{Au}, \mathrm{Ag}, \mathrm{Ni}, \mathrm{Co}, \mathrm{Fe}, \mathrm{Fe}-\mathrm{Ni}$ and so on. ${ }^{3}$ The silica thickness from 20 to $100 \mathrm{~nm}$ can be adjusted by controlling the experimental parameters such as coating time, concentration of reactions, catalyst, and other precursors. Silica-coated magnetite particles are well dispersed and more biocompatible when used for biological applications. 
In nanoscience and nanotechnology, the word "silica" is used to broadly refer to a variety of different materials that are derived from the hydrolysis of siloxanes. ${ }^{4}$ While some researchers even use the formula " $\mathrm{SiO}_{2}$ ", it is well known that the silica materials contain many dangling bonds, not to mention impurities such as $\mathrm{H}_{2} \mathrm{O}, \mathrm{NH}_{3},{ }^{5}$ ions, ${ }^{6}$ dyes, ${ }^{7}$ and surfactants. ${ }^{8}$ Generally speaking, the paradigm is to view the materials as silica-centered hybrids: the silica is the dominant framework onto which various types of impurities are simply adsorbed. As such, the impurities are expected to play minor roles in the formation and disintegration of the framework.

Another common misconception is that the silica formed in solution should have similar properties as those derived from molten conditions (sand, glass, etc.). Thus, silica nanomaterials are generally considered as densely crosslinked and insoluble, which is in conflict with the rapid etching of silica nanoparticles under neutral or mildly basic conditions. ${ }^{6}$ Such chemical instability poses problems for applying silica nanomaterials in biomedical research. ${ }^{9-11}$ While it is well known that the silica nanoparticles are microporous, ${ }^{10-14}$ the origin of the porosity and its effects on the silica property have not been extensively investigated.

There have been quite a few reports on the hollowing of the typical silica nanoparticles upon etching, ${ }^{15-18}$ but it remains elusive why their interior is removed preferentially. This facile route to hollow nanostructures has been extensively exploited in applications. ${ }^{19,20}$ Given the widespread use of silica and 
other oxides in nanotechnology, a better understanding of their structure and property is of crucial importance. The common views of silica are in obvious conflict with the phenomena of hollowing. Without selectivity, one would normally expect the etching to either cause a uniform loss of materials across the entire silica domain, or to dissolve the outmost layer first before reaching the inner layers. Several hypotheses have been put forward to explain the selectivity, involving either the redeposition of the dissolved materials ${ }^{15-17,21}$ or a certain inherent structural inhomogeneity (composition, ${ }^{22}$ grain size, ${ }^{19,23,24}$ degree of crosslinking, ${ }^{25}$ etc.). For clarity, these mechanisms can be grouped on the basis of se-lectivity: (1) The inner silica layer may be selectively removed because of its different composition; (2) The etching occurs uniformly but the re-deposition is selective, maybe because the particle-solvent interface has a special environment that somehow promotes re-deposition; or (3) The combination of the above two types of selectivity, for example, a certain structural difference may lead to selective Ostwald ripening, etching the inner and strengthening the outer layer.

While the pioneer studies have made importance ad-vances in our understanding, ${ }^{7,15,23-33}$ the origin of the selectivity remains elusive. Regardless of the hypotheses, further understanding is needed: what promotes the re-deposition at the silica-solvent interface; what is the origin of the structural inhomogeneity; and why cannot Ostwald ripening strengthen the inner layer at the cost of the outer layer? Resolving these fundamental questions can reveal the inner 
workings of silica, preempting problems and affording synthetic dexterity that is essential for the application of silica.

Our investigation in the origin of selective silica etching (Figure 1) leads to a paradigm shift in viewing the silica nanomaterials, where the counter ions play critical roles in the formation and etching of silica. The silica precursors in the solution can be viewed as crosslinked polyelectrolyte. Their nucleation/deposition depends on the extent of ion pairing and the size of the polymer, leading to two stages of nucleation. In comparison to the inner silica layer formed at the initial stage, the outer layer is made of shorter poly(silicic acid) chains with a lower extent of ion pairing. Thus, a higher degree of crosslinking makes the outer layer more resistant towards etching. The resulting silica nanoparticles, like polyelectrolyte aggregates, are highly swollen by the solvent and contain lots of trapped counter ions. When re-dispersed in water, these counter ions greatly promote the etching and ripening processes.

\subsection{Materials and methods}

\subsubsection{Materials}

All materials were used without further purification. Potassium chloride, potassium cyanide, tetramethylammonium hydroxide (TMAOH) were purchased from Aldrich; Hydrogen tetrachloroaurate(III) hydrate, 99\% (metal basis $\mathrm{Au}$ 49\%), sodium citrate dihydrate (99\%), and tetraethyl orthosilicate (TEOS), 3,7-bis(Dimethylamino)-phenothiazin-5-ium chloride (Methylene Blue), 
carboxyfluorescein, and poly(acrylic acid) (PAA) were purchased from Alfa Aesar; 11-mercaptoundeconoic acid (MUA) and 4-mercaptobenzoic acid (MBA) were purchased from Aldrich; Sodium hydroxide and $\mathrm{NH}_{3} \bullet \mathrm{H}_{2} \mathrm{O}$ (AR grade, 25-28\% w/w) were purchased from Sinopharm; 2-propanol (HPLC grade) was purchased from Fisher Scientific; $\mathrm{H}_{2} \mathrm{PtCl}_{6}, \mathrm{HAuCl}_{4}, \mathrm{H}_{2} \mathrm{PdCl}_{4}$, and Cisplatin (cis- $\left.\left[\mathrm{Pt}\left(\mathrm{NH}_{3}\right)_{2} \mathrm{Cl}_{2}\right]\right)$ were purchased from Sigma. Copper specimen grids (300 meshes) with formvar/carbon support film (TEM grids) were purchased from Beijing XXBR Technology Co.. Deionized (DI) water (resistance > $18.2 \mathrm{M}$ $\mathrm{cm} \square 1$ ) was used for the etching experiments; and HPLC water from Sigma was used in all silica syntheses.

Transmission electron microscopy (TEM) images were taken from a JEM-1400 transmission electron microscope (JEOL) operated at $100 \mathrm{kV}$. Ultraviolet-visible (UV-Vis) spectra were collected from Cary 100 spectrophotometer. Elemental analysis (EuroVector Euro EA Elemental Analyzer CHNS) and inductively coupled plasma mass spectrometry (ICP-MS) (Agilent 7700x) were used to characterize the elemental component and metal ion content within the silica nanoparticles. The Brunauer-Emmett-Teller (BET) measurements were carried out using Autosorb 6B; the surface area of the silica nanoparticles were calculated from $\mathrm{N}_{2}$ sorption at $77 \mathrm{~K}$ based on non-local density function theory model in ASiQwin software package. 


\subsubsection{Methods}

Characterization. Transmission electron microscopy (TEM) images were collected on a JEM-1400 (JEOL) operated at $100 \sim 120 \mathrm{kV}$. Field emission scanning electron microscopy (SEM) images were collected on a JEOL JSM-6700F. High-resolution TEM (HRTEM) images were taken from JEOL $2100 \mathrm{~F}$ field emission transmission electron microscopy operated at $200 \mathrm{kV}$.

Preparation of TEM Samples. TEM grids were treated with oxygen plasma in a Harrick plasma cleaner/sterilizer for $45 \mathrm{~s}$ to improve the surface hydrophilicity. The hydrophilic face of the TEM grid was then placed in contact with the sample solution. A filter paper was used to wick off the excess solution on the TEM grid, which was then dried in air for $30 \mathrm{~min}$.

Synthesis of Stöber silica. The typical synthesis of Stöber silica is as follow: $400 \mu \mathrm{L}$ of HPLC-grade $\mathrm{H}_{2} \mathrm{O}$ and $40 \mu \mathrm{L}$ of $\mathrm{NH}_{3}(28 \mathrm{wt} \%)$ were added into $1 \mathrm{~mL}$ of isopropanol under constant stirring (500 rpm). $2 \mu \mathrm{L}$ of neat tetraethoxylsilane (TEOS) was added and the mixture was incubated at room temperature with stirring for $12 \mathrm{hrs}$. The silica nanoparticles were isolated by centrifugation and purified for 3 cycles in water. The final product was redispersed in $\mathrm{EtOH}$ for further use.

Different water/isopropanol solvent ratios can be used. The relative volume of the solutions were adjusted, so that the final volume was $1.44 \mathrm{~mL}$ and solvent 
ratio was 1:20, 1:5, 2:5, and 3:5, respectively. All other conditions were kept the same.

Purification and standard etching experiment. The silica nanoparticles were isolated from the as-synthesized solution by centrifugation, purified in water for 3 cycles. In each cycle, the concentrated nanoparticle solution was dispersed in water and isolated by centrifugation. The residue volume after each step of centrifugation and removing the supernatant was about 6-8 $\mu \mathrm{L}$. For strict control experiments, the amount was set as exactly $8 \mu \mathrm{L}$ after topping up with the supernatant.

After purification, the silica nanoparticles were dispersed in $1 \mathrm{~mL}$ of water and the solution was incubated at $90{ }^{\circ} \mathrm{C}$ for $30 \mathrm{~min}$. This condition is defined as the standard etching condition. Typically, all of the silica nanoparticles of a batch of the typical synthesis (Section 1.1) were used for the etching experiment and the nanoparticle concentration was defined as $\mathrm{C}_{0}$.

As control experiments, the etching solvent was changed to water/isopropanol mixture, with the ratio being $1: 4$, to $1: 1,4: 1$, and $1: 0$, respectively. To vary the concentration of silica nanoparticles, scaled-up syntheses were carried out and solutions $\left(\mathrm{C}_{0} / 2, \mathrm{C}_{0}\right.$, and $\left.4 \mathrm{C}_{0}\right)$ were prepared from the isolated nanoparticles by controlling the dilution factors. For etching in basic solution, $1 \mathrm{~mL}$ of ammonia $(0.27 \mathrm{M})$ or $\mathrm{NaOH}(50 \mathrm{mM})$ were used in the place of pure water. 


\section{Synthesis of silica using different bases as the catalyst. $\mathrm{NaOH}$ and}

TMAOH can be also used as the catalyst. All other conditions were kept the same as Section 1.1 except that $\mathrm{NaOH}(2 \mathrm{mM})$ and TMAOH $(2 \mathrm{mM})$, respectively, were used in the place of $\mathrm{NH}_{3}$. The silica nanoparticles were purified for 3 cycles in water and stored in ethanol.

The reactions were scaled up by 20 times for the samples used for elemental analysis and BET measurements. After purification, the nanoparticles were isolated by centrifugation and the samples dried in oven for $6 \mathrm{~h}$.

Synthesis of Stöber silica in the presence of salt. $\mathrm{NaCl}, \mathrm{KCl}$ and $\mathrm{CsCl}$ were introduced in the Stöber synthesis (Section 1.1). Stock solutions of $100 \mathrm{mM}$ were prepared and aliquots were added to the $\mathrm{NH}_{3}$ solution in water/isopropanol, before the addition of TEOS, so that the final concentration was $2 \mathrm{mM}$. All other conditions were kept the same.

For Figure 5i-k, different amounts of $\mathrm{NaCl}$ stock solution were added, so that the final concentration was $0.8 \mathrm{mM}, 3 \mathrm{mM}$, and $15 \mathrm{mM}$, respectively. All other conditions were kept the same.

For ICP-MS measurements, the purified silica nanoparticles were dissolved in $5 \mathrm{~mL}$ of ammonia solution $(56 \mathrm{mM})$ and heated $90{ }^{\circ} \mathrm{C}$ for $30 \mathrm{~min}$. TEM characterization showed no silica nanoparticles after the etching. The solution was directly used for ICP-MS. 
For EDX line scan, the purified silica nanoparticles were dispersed in $\mathrm{EtOH}$ and then dried on copper grids.

\subsection{Results and discussion}

As discussed above, the key issue in the hollowing me-chanism is to understand the origin of selectivity and relate it to the process of silica formation.

Interface by itself is not a good explanation because it is not unique. For any silica nanoparticle, there are several types of silica-solvent interfaces: the interface with the outside solution; the interface with the solution inside the hollow cavity once it forms; and the interface with the solution inside the micropores of silica. During silica etching/ripening, the dissolved material is more concentrated inside than outside the silica nanoparticles. Hence, there seems to be no clear chemistry basis for the preferential deposition at the outer silica-solvent interface. There is also no indication that the dissolved silica species may be reacting with the "fresh" solvent/solutes outside.

Ostwald ripening is known to make small nanocrystals smaller and large ones larger, driven by the difference in their surface energy. ${ }^{21,34}$ Because silica is amorphous, the individual nanoparticles cannot be treated as a whole. The ripening concept should be applied to the "grains" within the amorphous domain that have different sizes and/or degrees of crosslinking. But the ripening process by itself does not give rise to the selectivity in hollowing. Unless one assumes that the various layers of the silica nanoparticles inherit their different degrees of 
crystallinity from the initial synthesis, the small and large "grains" should distribute uniformly within the nanoparticles. Upon ripening, fluctuation of grain sizes would eventually lead to coarse grains across the entire silica domain. This expectation is inconsistent with the hollowing phenomena.

Bases such as $\mathrm{NH}_{3}$ and $\mathrm{NaOH}$ can catalyze the hydrolysis of siloxanes and/or the crosslinking of silica, ${ }^{35}$ but the promotion of these reactions does not lead to the selectivity in hollowing. Similarly, there is no selectivity when dissolving silica in these bases. As long as the compositions are similar, the bases should have roughly the same effects on the different parts of the silica nanoparticles. Indeed, if diffusion is the limiting factor, the outmost layer should be most affected.

Our thesis is that ion pairing plays important roles in the formation of amorphous silica nanoparticles, causing compositional differences among the layers. To reduce ambiguity, we tried to minimize the ingredients present in the silica growth, avoiding the use of surfactants, buffers, and unnecessary oxide precursors. The silica properties were probed by studying their hollowing in water and by characterizing the incorporated ions.

To set the stage, we need to understand why silica can dissolve in water. It is known that quartz has a solubility of about $11 \mathrm{ppm}$ at $25^{\circ} \mathrm{C},{ }^{1}$ and it requires hundreds of days to reach this equilibrium. The fact that the Stöber silica can be 
dissolved in aqueous solution is a first indication for its different chemical composition.

\subsubsection{Synthesis and etching}
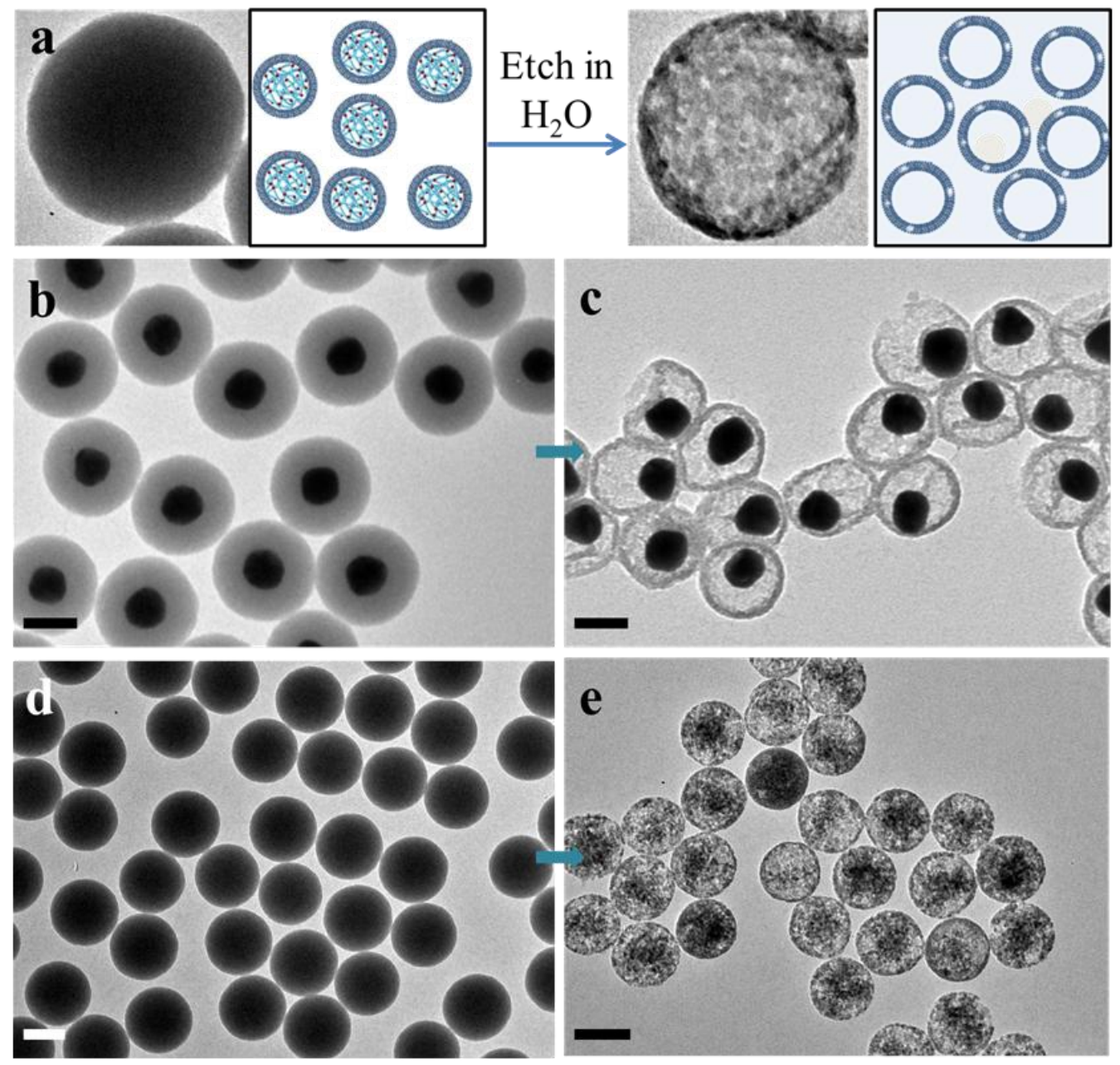

Figure 2.1. (a) Schematics and TEM images of silica nanoparticles before and after etching in water. The silica domain appears uniform in contrast but its inhomogeneous composition is only revealed after etching. TEM images of Au@silica nanoparticles (b) before and (c) after etching; and silica nanoparticles (d) before and (e) after etching. Scale bar: $50 \mathrm{~nm}$. Reprinted with permission from ref. [28], respectively, copyright 2015 Wiley-VCH. 
In the following experiments, the silica nanoparticles were prepared by the traditional Stöber method, 2 where the only ingredients present were the solvent (water : isopropanol $=2: 5)$, NH3 $(0.47 \mathrm{M})$, and TEOS $(9.1 \mathrm{mM})$. The resulting nanoparticles (the ammonia-catalyzed silica) were isolated by centrifugation and the concentrated solution was diluted in water. As shown in Figure 1d, the transmission electron microscopy (TEM) images of the silica nanoparticles showed sharp boundaries and uniform dark interior. When they were dispersed in water and heated at $90 \square \mathrm{C}$ for $30 \mathrm{~min}, 3$ they became hollow (Figure 2.1e, more strict experiments are carried out in the following). Similarly, Au@silica core-shell nanoparticles can be synthesized (Figure 2.1b) and then converted to yolk-shell nanoparticles via the same hollowing process (Figure 2.1c). More specifically, citrate-stabilized Au nanoparticles $(\mathrm{d}=40 \mathrm{~nm})$ were treated with 11-mercaptoundecanoic acid (11-MUA) to render their surface amenable to silica adsorption. They were then used as seeds in the Stöber method as previously reported. ${ }^{3}$ The product Au@silica nanoparticles (Figure 2.1b) were isolated by centrifugation and then similarly etched, giving yolk-shell 
nanoparticles. The advantage in using the Au@silica nanoparticles is
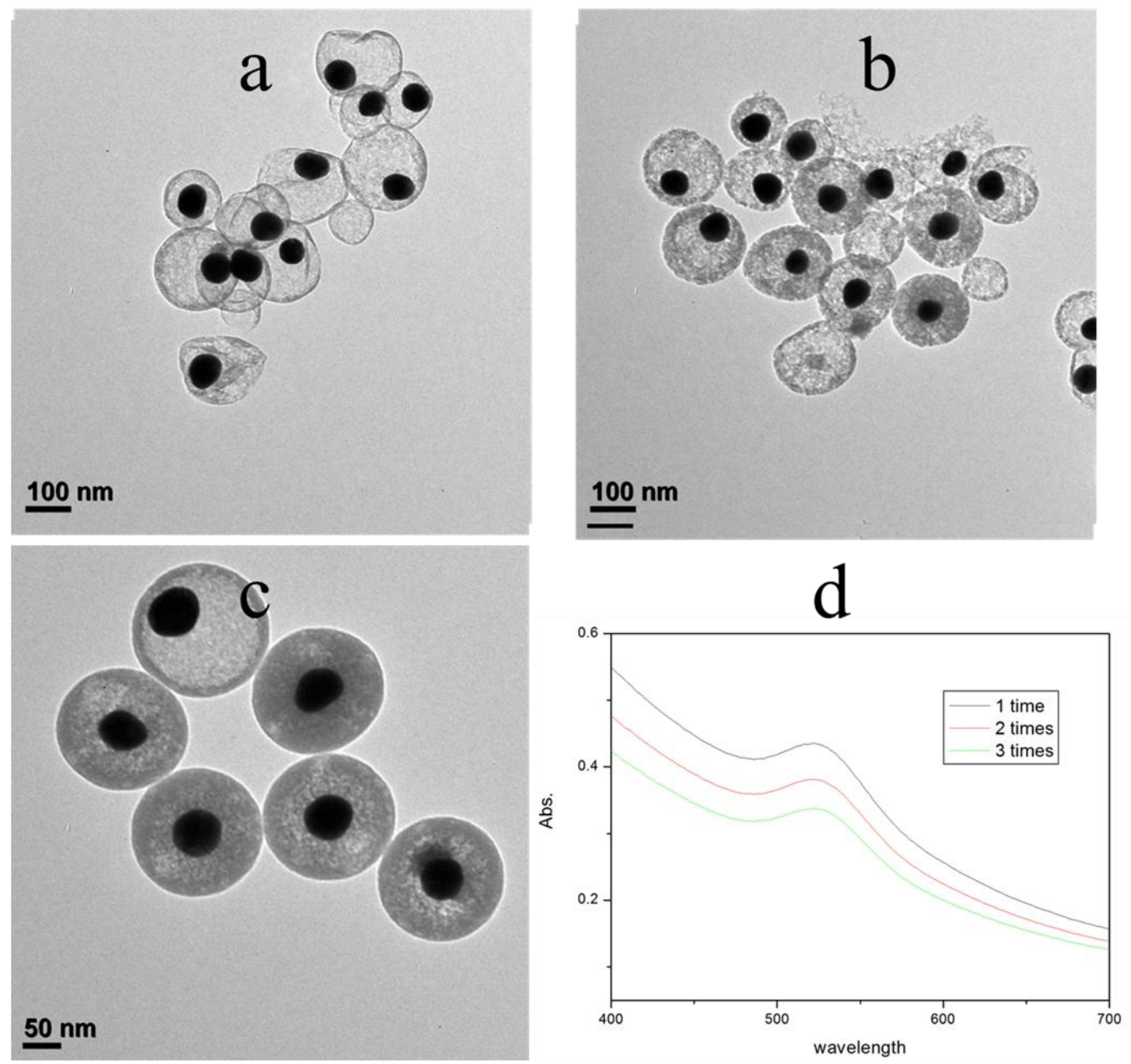

Figure 2.2. Au@silica nanoparticles were purified for different rounds: (a) N=1, (b) $\mathrm{N}=2$, (c) $\mathrm{N}=3$. The samples were etched in $90{ }^{\circ} \mathrm{C}$ water for $30 \mathrm{~min}$ and the TEM images of the resulting nanoparticles were shown. (d) UV-Vis spectra of these samples after the purification, showing that the loss of nanoparticles during the purification was minimal.

that the red color of the nanoparticle solution can be easily tracked during the multi-step centrifugation and re-dispersion. By following the UV-Vis 
absorption of the solutions, ${ }^{4}$ we can be certain that there is minimal loss of the nanoparticles and that they are not aggregating.

$\mathrm{NaOH}$ and tetramethylammonium hydroxide (TMAOH) can also be used in the place of $\mathrm{NH}_{3}$ for synthesizing silica and Au@silica nanoparticles (the $\mathrm{NaOH}-$ and TMAOH-catalyzed silica). The resulting nanoparticles were similarly etched. ${ }^{4}$

\subsubsection{Understanding silica dissolution in water}

In neutral aqueous solutions, it is well known that quartz $\left(\mathrm{SiO}_{2}\right)$ is insoluble. ${ }^{5-7}$ After extensive experiments, we realized that the etching of silica nanoparticles in aqueous solutions is essentially a matter of solubility. It is just that the chemical composition of the "silica" is different, in contrast to quartz which is exactly $\mathrm{SiO}_{2}$ with maximized degree of "crosslinking". Here, we use the word "silica" in a general sense to be consistent with the literature convention. In this section, we focus on the dissolution of the silica interior. The difference in etching the outmost silica shell will be addressed in the following sections.

Typically, the etching solution contained some residue base $\left(\mathrm{NH}_{3}\right.$ or $\left.\mathrm{NaOH}\right)$, which was part of the Stöber method and the leftover of the purification process. To strictly control the amount of residue base, the isolated nanoparticle solution after each step of centrifugation was set to be exactly $8 \mu \mathrm{L}$ (by topping up with the supernatant), before it was diluted in $1 \mathrm{~mL}$ of water. Thus, the residue $\left[\mathrm{NH}_{3}\right]$ 

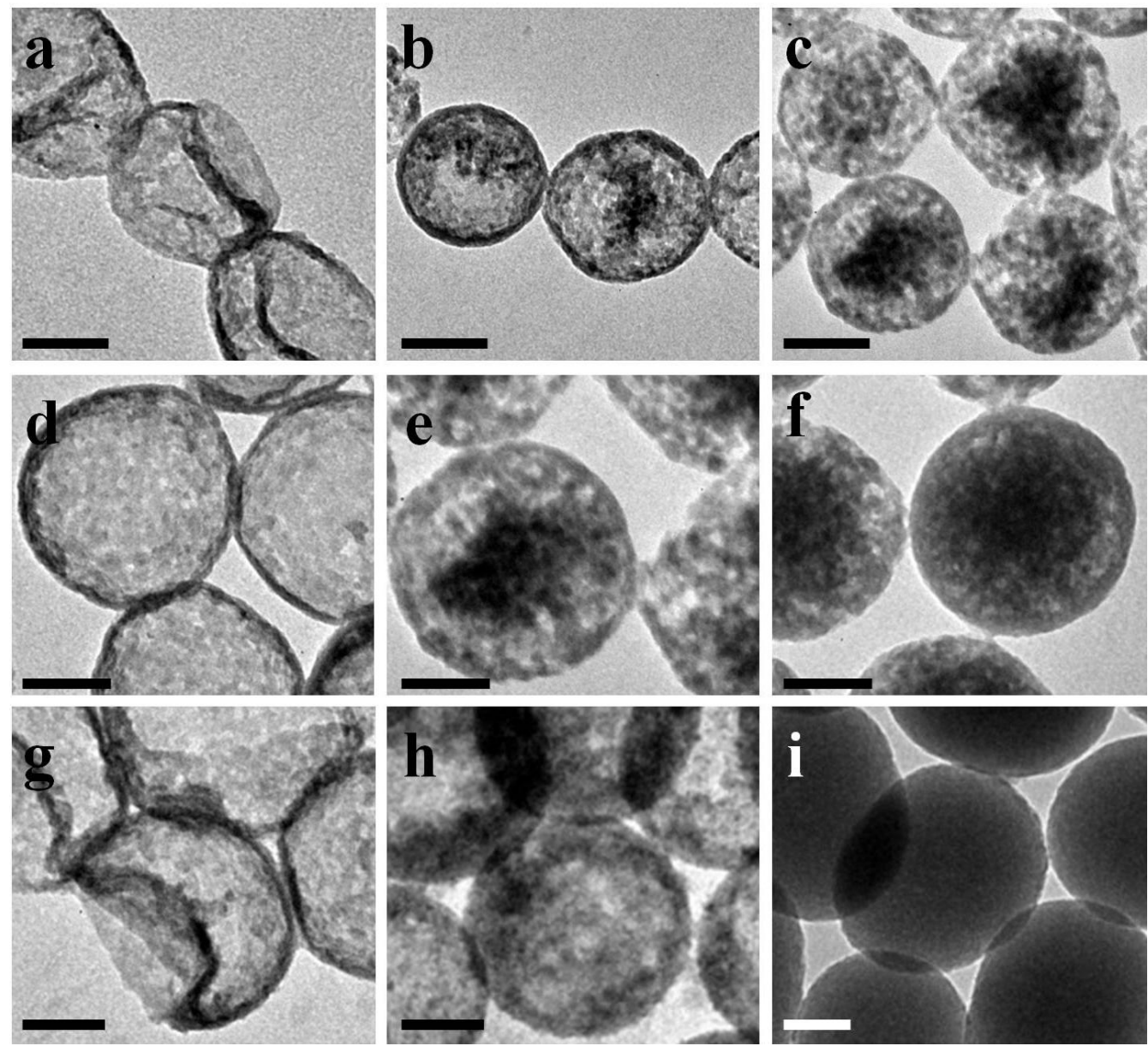

Figure 2.3. TEM images showing the results of etching Stöber silica in $90{ }^{\circ} \mathrm{C}$

water. (a-c) With more purification cycles: (a) $\mathrm{N}=1$; (b) $\mathrm{N}=2$; (c) $\mathrm{N}=3$, less

residue base was brought into the etching solution (pure water), reducing the extent of etching. (d-f) After being purified for 3 times, different amounts of silica nanoparticles were etched in water: (d) $\mathrm{C}_{0} / 2$; (e) $\mathrm{C}_{0}$; (f) $4 \mathrm{C}_{0}$. The concentration of silica nanoparticles used in a-c and g-i were all $\mathrm{C}_{0}$. (g-i) Silica nanoparticles were purified once and then etched in different solvents: (g) water/isopropanol =1:0; (h) 1:1; (i) 1:4. Scale bar: $50 \mathrm{~nm}$. Reprinted with permission from ref. [28], respectively, copyright 2015 Wiley-VCH.

in the sample after the $1^{\text {st }}, 2^{\text {nd }}$, and $3^{\text {rd }}$ cycle of purification (i.e., centrifugation 
followed by the dilution) was $3.6 \mathrm{mM}, 29 \mu \mathrm{M}$, and $0.23 \mu \mathrm{M}$, respectively. Further cycles of purification did not lead to noticeable difference in etching, indicating that the remaining residue can be ignored. These samples were etched in water at $90{ }^{\circ} \mathrm{C}$ for $30 \mathrm{~min}$. As shown in Figure 2.3a-2.3c, the degree of etching was greatly reduced when the amount of the residue $\mathrm{NH}_{3}$ was decreased. The shells appeared less folded and there were more remnants in the interior.

As we reduce the amount of residue $\mathrm{NH}_{3}$, the $\mathrm{pH}$ of the solution is expected to change. The best way to control $\mathrm{pH}$ is to use buffer solutions. In our system, however, buffers are not suitable because the ions of the buffer would be incorporated in the silica nanomaterials. To minimize unnecessary complication, we used the commercially available HPLC grade water in all of our experiments and precisely controlled the dilution factors to achieve consistent etching conditions.

A same batch of silica nanoparticles after 3 cycles of purification was adjusted to different concentrations, by adjusting either the amount of the silica nanoparticles (Figure 2.3d-2.3f) or the amount of water (Figure 2.3). ${ }^{4}$ A clear trend emerged that a lower concentration of the silica nanoparticles led to more extensive etching. Basically, with less nanoparticles present, more can be dissolved from each nanoparticle. Considering the absence of base in these samples, the fact that the silica nanoparticles can be extensively etched in water 
was surprising. In particular in Figure $2.3 \mathrm{~d}, 80 \%$ of the silica nanoparticles have been dissolved. On the basis of the total TEOS used for their synthesis, the silica solubility in the solution was estimated to be about $430 \mathrm{ppm}(7.2 \mu \mathrm{M})$, over 40 times larger than that of quartz and also much higher than the residue base $(0.23 \mu \mathrm{M})$ present. The microporosity of Stöber silica may facilitate its rate of dissolution, but its high solubility cannot be explained otherwise. This is a first indication that the composition of the Stöber silica is different from that of quartz.

After centrifugation to remove the undissolved hollow nanoparticles, the supernatant was collected, presumably saturated with the dissolved form of silica. Using this solution as the solvent, attempts to etch a new batch of silica nanoparticles failed (Figure 2.3h). No sign of etching was observed when the etching time was extended to $2 \mathrm{~h}$ (Figure 2.3i). In another set of experiments, TEOS was hydrolyzed in water for $8 \mathrm{~h}$ and the "saturated" solution was used as the solvent; silica nanoparticles were not etched as shown in Figure 2.3g. The fact that silica nanoparticles can be dissolved in water but not in a saturated solution suggests that there is indeed solubilization equilibrium. Importantly, these results also argue against the surface redeposition and Ostwald ripening as the hollowing mechanism (vide infra).

The quality of the etching solvent was also of importance. When the purified silica nanoparticles were dispersed in water/isopropanol mixture 

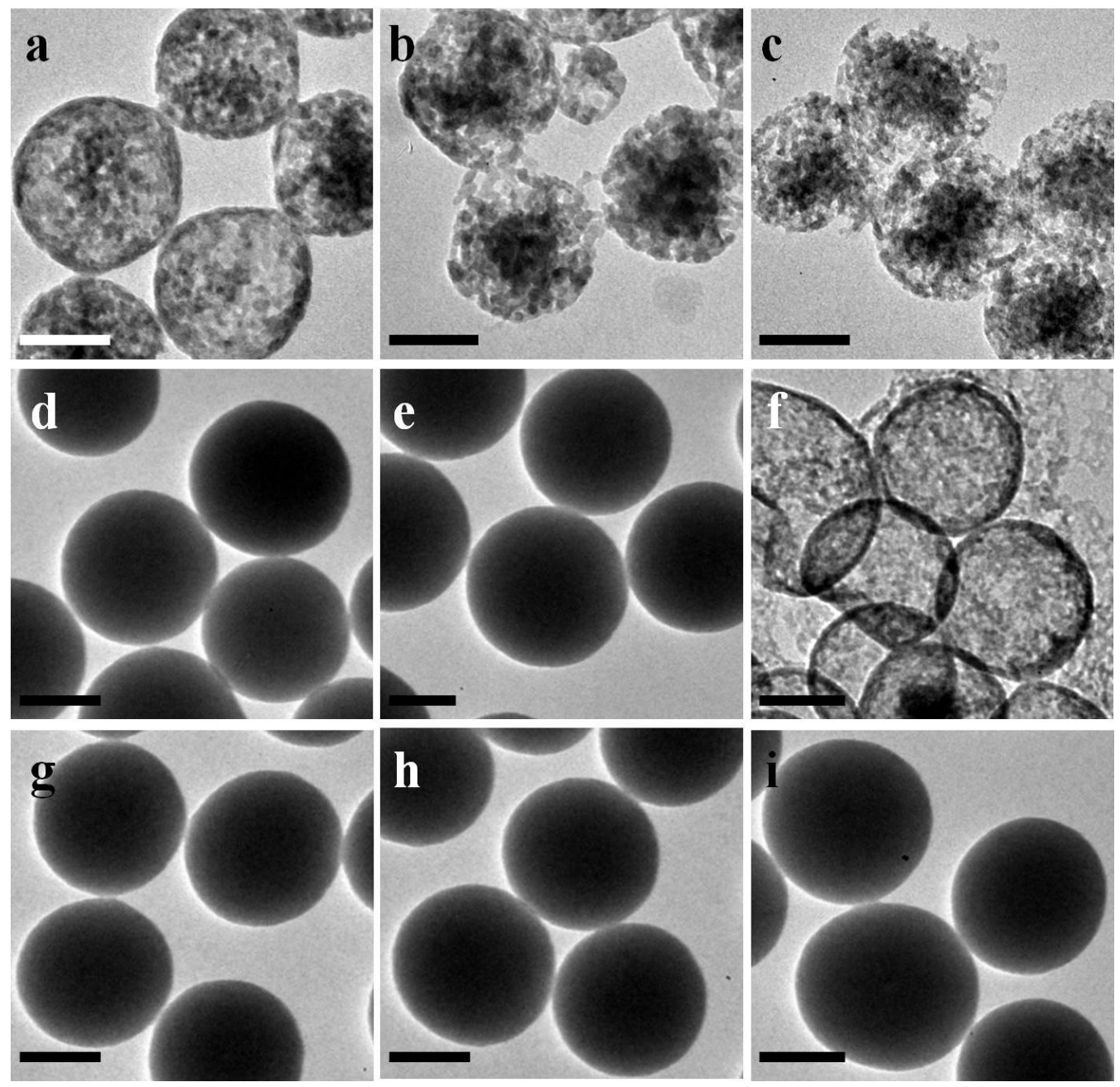

Figure 2.4. (a-c) TEM images showing the different degrees of ripening in the

silica nanoparticles after etching in $90{ }^{\circ} \mathrm{C}$ water for (a) 30; (b) 60; and (c) 90 min. (d-f) Silica nanoparticles were purified for 3 times, treated in $2 \mathrm{M} \mathrm{HCl}$ for 30 min, and then purified for 3 times to remove the acid: (d) the product nanoparticles; (e) after etching sample d in $90{ }^{\circ} \mathrm{C}$ water for $30 \mathrm{~min}$; and (f) after etching sample $\mathrm{d}$ in $0.27 \mathrm{M}$ ammonia solution at $90{ }^{\circ} \mathrm{C}$ for $30 \mathrm{~min}$. (g-i) Control experiments showing that etching cannot occur in solutions pre-saturated with silica. The nanoparticles after etching were shown: (g) TEOS was added in water; the mixture was incubated overnight before being used as the etching 
solvent; (h,i) after the standard etching experiment (Figure 2.4c), the residue nanoparticles were removed by centrifugation and the supernatant was used as the etching solution for a new batch of silica nanoparticles. The etching was carried out at $90{ }^{\circ} \mathrm{C}$ for (h) 30; and (i) $120 \mathrm{~min}$. Scale bar: $50 \mathrm{~nm}$. Reprinted with permission from ref. [28], respectively, copyright 2015 Wiley-VCH.

instead of pure water, the same treatment at $90^{\circ} \mathrm{C}$ gave much less etching. The silica was barely dissolved in water:isopropanol $=1: 4$ solution (Figure $2.4 \mathrm{i}$ ) and the etching became more extensive when the water content was increased (Figure 2.4g-h).

Hence, the extent of silica etching can be directly correlated to the amount of base present in the solution, to the amount of etching solvent, and to the quality of the solvent. These factors may either (1) promote the rate of silica dissolution; or (2) lead to a higher solubility of the silica. Control experiments showed that the degrees of etching as shown in Figure $2.3 \mathrm{a}, 2.3 \mathrm{~d}$, and $2.3 \mathrm{~g}$ barely changed when the etching time was extended from 30 to 90 min, indicating that near equilibrium conditions have been attained and thus the dissolving rate was not the limiting factor. Hence, the critical factor was the solubility of the "dissolved silica". Here, we do not distinguish the various soluble forms of silicic acid monomer, the oligo(silicic acid) and their anions. It is expected that many species would coexist with different degrees of ionization. ${ }^{8}$ Their total 
solubility is defined as the solubility of the "dissolved silica", which depends on the solvent quality and the base concentration. ${ }^{9}$

\subsubsection{Ostwald ripening}

As discussed above, ripening makes larger grains larger at the cost of smaller grains. The extent of ripening within the amorphous silica domain can be directly observed using TEM. In some cases, the outmost silica shell appeared uniform in contrast (Figure 2.4b, and 2.4e) and in others the shells were more coarse with mixed patches of dark and white (Figure 2.3c, 2.3f, 2.4b and 2.4c). Such patches are easily discernable at the center of nanoparticles, in contrast to the edge where the grains overlap with each other under the TEM projection. The etched shells with uniform contrast were usually soft (Figure 2.3b, 2.3e and 2.3h), manifesting wrinkles as a result of their folding. In contrast, the shells with larger grains appeared to be harder (Figure 2.4b, 2.4c), always maintaining the original spherical shape.

In Figure 2.3d-f, a same batch of silica nanoparticles was etched under similar conditions and yet their final grain sizes were obviously different. Thus, this difference was likely a result of the ripening process, not the heritage of the initial synthesis. It appeared that the degree of ripening depended on whether the dissolved silica reached saturation. At low nanoparticle concentration (Figure 2.3d) before the dissolved silica reached saturation, dissolution was the main process and thus the shells with small grains were obtained. In contrast, at 
higher nanoparticle concentrations (Figure 2.3e, and 2.3f), the solution quickly reached saturation, promoting ripening and giving shells with large grains.

Previously, the contact with external solvent was thought to improve the crystallinity of the outmost layer of silica/oxide. ${ }^{10,11}$ This initial difference then led to hollow nanoparticles upon extensive ripening. To test this hypothesis, we attempted to ripen silica nanoparticles in a solution pre-saturated with silica (Figure 2.4g-i, vide supra), which would stop silica dissolution but promote ripening. As observed, the absence of hollowing in these silica nanoparticles suggested that ripening in itself cannot lead to hollowing, contradicting with the ripening hypothesis.

\subsubsection{Hypothesis of silica formation}

We found that it is nearly impossible to explain the above phenomena if we assume all portions of the silica nanoparticles to have the same chemical composition. Should this be true, the thickness of the remaining shell would depend on the etching conditions and correlate to the extent of etching/ripening. The fact that shells of more or less the same thickness were obtained in Figure 2.3 and 2.4 suggests that it is influenced more by the initial synthesis than by the etching conditions, contradicting with the assumption of homogeneous composition.

These phenomena can be easily explained if we postulate different silica compositions within each silica nanoparticle. The hypothesis started from the 
realization that the soluble form of the silica precursors are essentially poly-electrolytes, ${ }^{12,13}$ albeit more crosslinked ones. More specifically, as TEOS

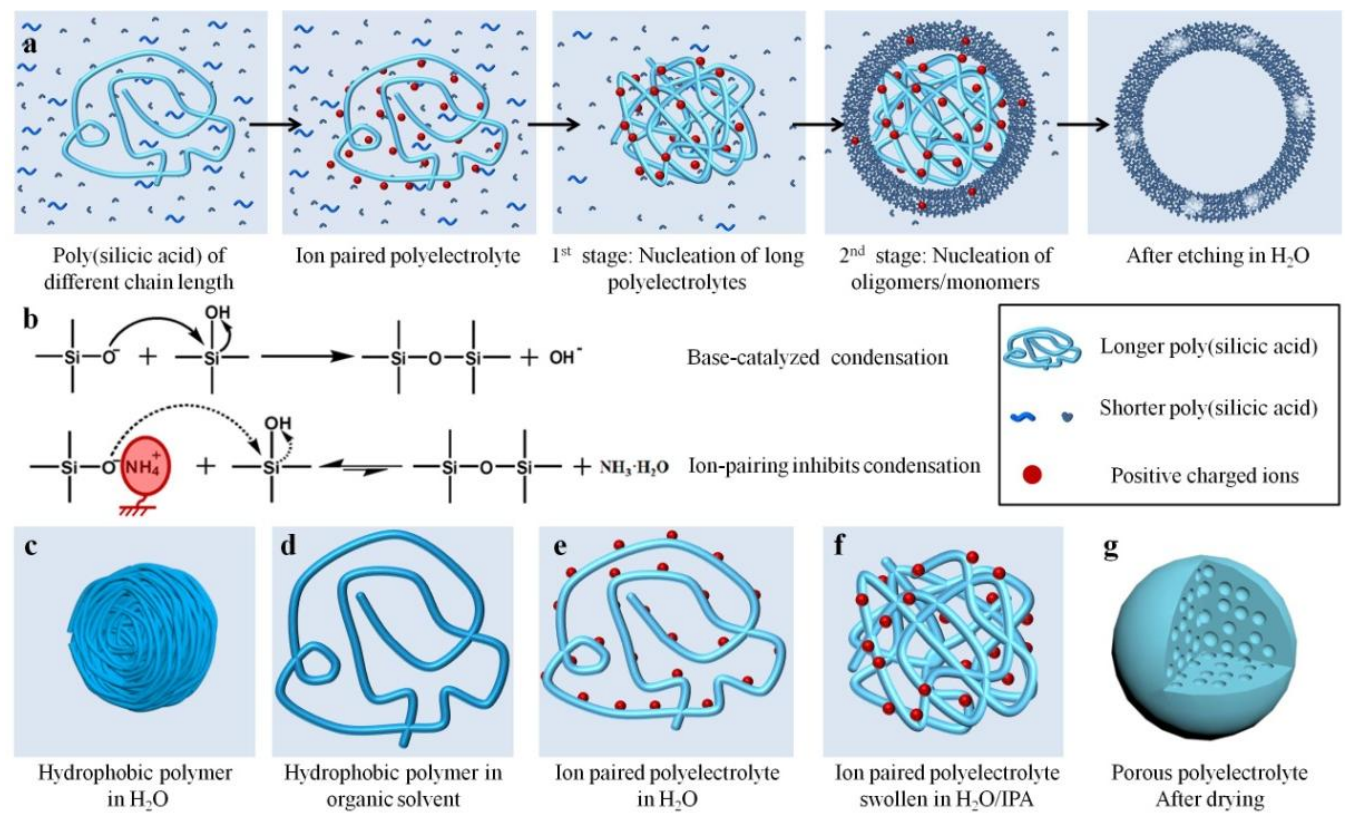

Figure 2.5. Schematics illustrating the significant role of ion pairing in the formation and etching of silica nanoparticles: (a) Ion pairing reduces the solubility of long poly(silicic acid) chains, more so than short oligomers do. These long polyelectrolytes preferentially nucleate in the first stage, trapping in with them lots of counter ions. In the second stage, the monomers and short oligomers nucleates on the nanoparticle surface, forming a more cross-linked shell. The silica domain with trapped ions can be selectively removed by etching in water. (b) Ion pairing inhibits the cross-linking of poly(silicic acid) chains. Normally, a few $\mathrm{OH}^{-}$ions can diffuse around and catalyze the condensation reaction. In contrast, with lots of anchored counter ions, the resulting base $\left(\mathrm{NH}_{3} \cdot \mathrm{H}_{2} \mathrm{O}\right)$ can cause reverse reactions and they are also responsible for the selective etching. (c-g) Schematics illustrating the concept of swelling: (c) 
hydrophobic polymer forms a close-packed domain in water; (d) hydrophobic polymer forms a loose dissolved state in an organic solvent; (e) polyelectrolyte with ion pairing at a soluble state in water; (f) long polyelectrolyte with ion pairing is insoluble in water/isopropanol mixture but swollen by the polar solvent; (g) after drying the nanoparticles of polyelectrolyte, micropores will result from the original solvent pockets. Reprinted with permission from ref. [28], respectively, copyright 2015 Wiley-VCH.

hydrolyzes in water, the resulting $-\mathrm{SiOH}$ groups can polymerize via condensation reactions or deprotonate giving negative charges. Thus, the resulting poly(silicic acid) can attract counter ions just like any other polyelectrolytes. $^{14}$

In the literature, it is well known that DNA and RNA, being polyelectrolytes, can be readily precipitated with salt in water/ethanol mixture. By modulating the solvent polarity, DNAs of different chain length can be separated. ${ }^{15-20}$ Similarly, $\mathrm{NH}_{3}$ can induce the precipitation of poly(acrylic acid) in ethanol/water mixture, giving polymer nanoparticles. ${ }^{4,13}$ In general, longer polyelectrolytes are less soluble under the same conditions. ${ }^{21,22}$ Basically, poly-ions in water are normally solvated because of the large solvation energy, which would be greatly reduced with the addition of the less polar ethanol. Thus, ion pairing becomes more favorable because the electrostatic potential exceeds the solvation energy. In comparison to individual ions and short 
oligomers, ion pairing within long and coiled polyelectrolytes can achieve multiple points of ionic interactions without entropic penalties. ${ }^{23,24}$ Thus, long polyelectrolytes tend to aggregate and contract, leading to their precipitation/nucleation from the solution. ${ }^{25,26}$

It should be noted that it is hard to synthesize Stöber silica in pure water. Ethanol or isopropanol is always added to reduce solvent polarity and the reaction is often carried out in the presence of a base $\left(\mathrm{NH}_{3}\right.$ or $\left.\mathrm{NaOH}\right)$, which provides sufficient concentration of counter ions $\left(\mathrm{NH}_{4}{ }^{+}\right.$or $\left.\mathrm{Na}^{+}\right)$. On these bases, we postulate that the precipitation of poly(silicic acid) follows the same principles, that ion pairing is essential for its nucleation; and that long poly(silicic acid) chains nucleate before the short ones do. With this new view, there are three important consequences, namely the sequential precipitation of the poly(silicic acid) of different lengths, the lack of cross-linking within the silica domain, and the swelling by solvent.

As illustrated in Scheme 2.5b, normally $\mathrm{OH}^{-}$ions can diffuse around and can catalyze the condensation reaction, leading to cross-linking of poly(silicic acid) chains, but the trapped counter ion can prevent it. Once $\mathrm{NH}_{4}{ }^{+}$ion is wrapped inside, its multiple $\mathrm{H}$-bonding with the neighboring - $\mathrm{SiOH}$ groups and the strong charge attraction between the ion pair would greatly reduce its ability to diffuse. The $-\mathrm{SiO}^{-} \mathrm{NH}_{4}{ }^{+}$ion pair is equivalent to one released $\mathrm{NH}_{3}$ molecule after the condensation reaction. Thus, the large amount of "base" trapped inside 
can reverse the effects of cross-linking. Such trapped "base" is expected to make the Stöber silica more soluble and lead to the preferential hollowing of the silica nanoparticles.

As is typical for polyelectrolytes, the solubility of the ion-paired poly(silicic acid) should depend on the polymer chain length, which is variable at the different stage of silica formation. Considering the large concentration of TEOS at the initial stage $(0-4 \mathrm{~h})$, it is expected that the long poly(silicic acid) would form. They would preferentially nucleate due to their low solubility, wrapping in with them a large number of $\mathrm{NH}_{4}{ }^{+}$counter ions. In contrast, the outer silica shell was build up from the subsequent addition of the leftover monomers and short oligo(silicic acid). These shorter polyelectrolytes retain less counter ions and eventually give more crosslinked silica shell. Hence, the dramatic difference between the two stages leads to an abrupt boundary of different compositions (as opposed to a smooth radial gradient), ${ }^{3}$ which is manifested as a clean shell after the etching. It explains why the etching of the inner layer does not start from the center of the nanoparticle, but starts as small voids next to the outmost layer (Figure 2.6f). ${ }^{11}$ In other words, the selectivity in etching originates from the silica inhomogeneity, which in turn originates from the length difference of the poly(silicic acid) during the synthesis.

Considering the polar - $\mathrm{SiOH}$ groups and ionic species, the silica polymer should be highly swollen by the polar solvent molecules. In polymer science, a 

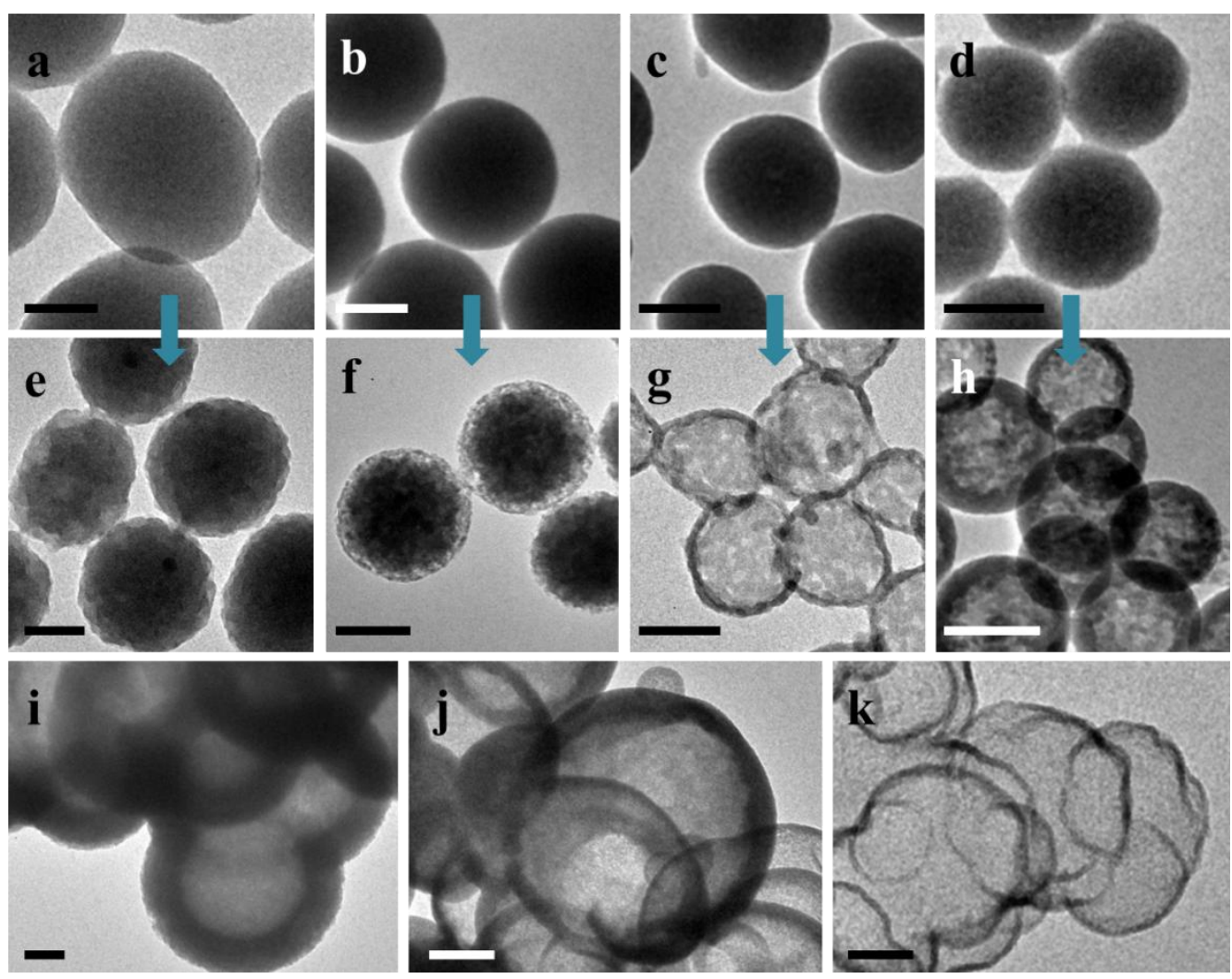

Figure 2.6. TEM images showing the modulation of composition in silica nanoparticles. (a-h) Silica nanoparticles were synthesized in different water/isopropanol mixture: (a) 1:20; (b) 1:5; (c) 2:5; (d) 3:5. These samples were purified 3 times and then etched in pure water at $90{ }^{\circ} \mathrm{C}$ for $30 \mathrm{~min}$, and the resulting nanoparticles were shown in (e-h), respectively. (i-k) Silica nanoparticles were synthesized in water/isopropanol mixture (1:5) in the presence of $\mathrm{NaCl}$ : (i) 0.8; (j) 3; and (k) $15 \mathrm{mM}$. Scale Bar: $50 \mathrm{~nm}$.

piece of rubber immersed in gasoline is known to expand in size, due to the favorable interactions (the solvation energy) between the solvent molecules and the polymer chains. ${ }^{27}$ This principle also applies to polyelectrolytes such as sodium polyacrylate (filler materials of typical diapers), which can absorb huge 
proportion of water (up to 300 times the mass of the polymer) due to the strong solvation energy of the ions. The swelling of the Stöber silica essentially means that the water molecules surrounding the $-\mathrm{SiOH},-\mathrm{SiO}^{-}$, and $\mathrm{NH}_{4}{ }^{+}$groups would demand a sizeable space. Given the immobile silica framework, lots of empty space will result after drying (Scheme 2.5c). Thus, the well-known microporosity of the silica formed in polar solvents (as opposed to the silica formed at molten conditions) is consistent with the hypothesis of ion pairing.

With these analyses, the drastically different etching behavior of the inner and outer layers can be easily understood. The fact that the outer shell thickness is independent of the extent of etching (Figure 2.3 and 2.4) can be explained.

\subsubsection{Analysis of silica composition}

On the basis of the above analysis, we studied the silica composition to examine our hypothesis.

The silica nanoparticles prepared by the Stöber method were purified in water for a variable number of cycles, and then dried in oven for $6 \mathrm{~h}$. The samples were then weighted and subjected to elemental analysis (CHNS method and inductively coupled plasma mass spectrometry, ICP-MS). As shown in Figure 2.7a, both the $\mathrm{N}$ and $\mathrm{C}$ content decreased during the 3 cycles of purification, but they remain roughly unchanged for the samples with 3-5 cycles of purification. Since $\mathrm{NH}_{3}$ was the only source of $\mathrm{N}$ in the reaction, the $\mathrm{N}$ content in the silica 

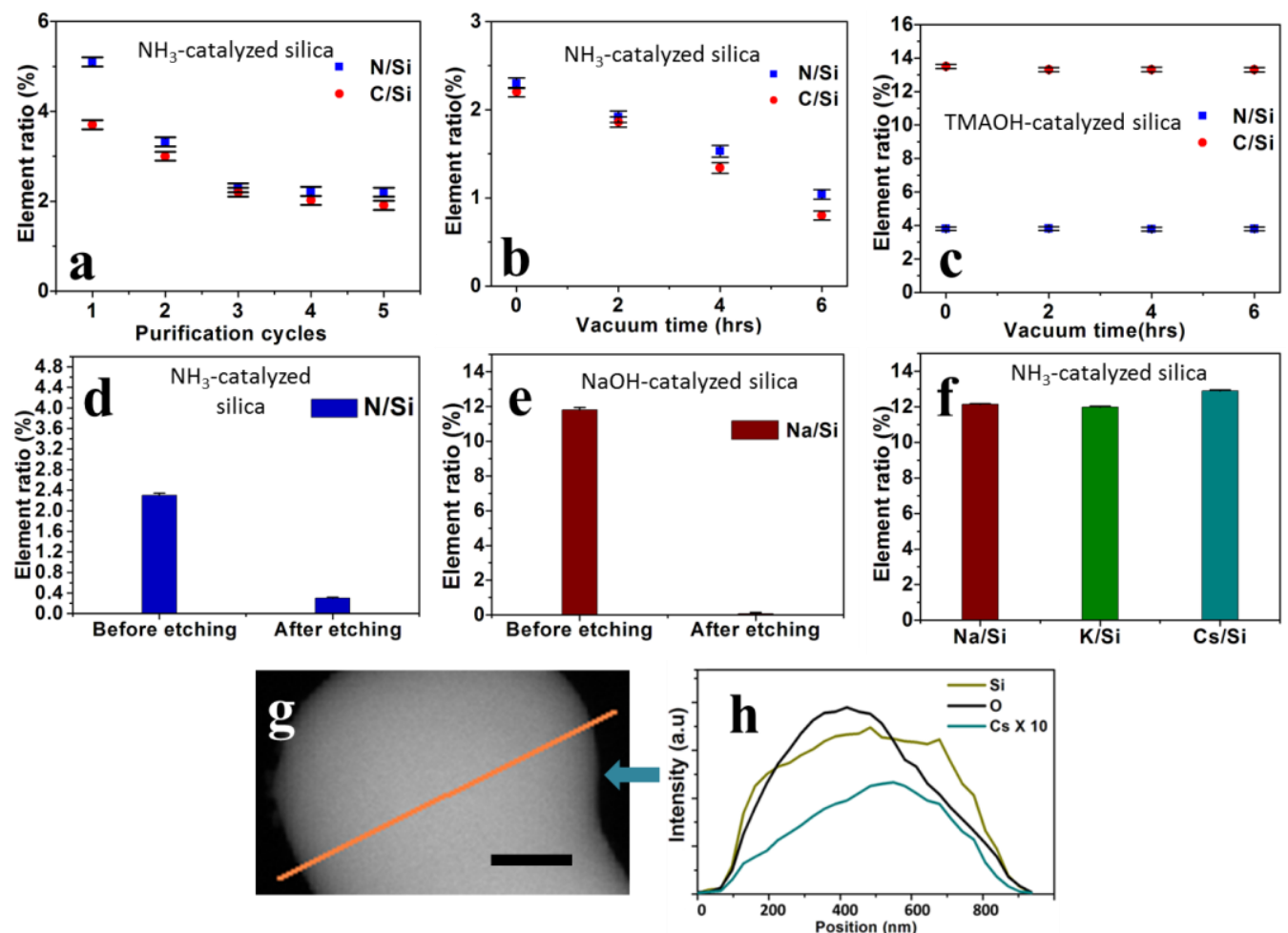

Figure 2.7. Elemental analyses proving the presence of trapped ions. (a-d) The elemental analysis (CHNS) and (e-f) ICP-MS results are presented using element/Si ratio: (a) Ammonia-catalyzed silica samples were purified for 1-5 cycles and dried in oven. (b) Ammonia-catalyzed silica samples were purified for 3 cycles, dried in oven, and then subjected to vacuum treatment for 0-6 h. (c) TMAOH-catalyzed silica samples were purified for 3 cycles, dried in oven, and then subjected to vacuum treatment for 0-6 h. (d) Ammonia-catalyzed silica sample before and after etching. (e) $\mathrm{NaOH}$-catalyzed silica sample before and after etching. (f) Ammonia-catalyzed silica samples synthesized in the presence of $\mathrm{NaCl}, \mathrm{KCl}$, and $\mathrm{CsCl}$ were purified for 3 cycles and then dried in oven. (g-h) EDX line scan for the CsCl-doped silica in f. Scale bar: $50 \mathrm{~nm}$.

can be attributed to the embedded $\mathrm{NH}_{4}{ }^{+}$ions. The $\mathrm{C}$ content may result from the 
trapped solvent isopropanol or the un-hydrolyzed ethoxyl groups from the initial TEOS.
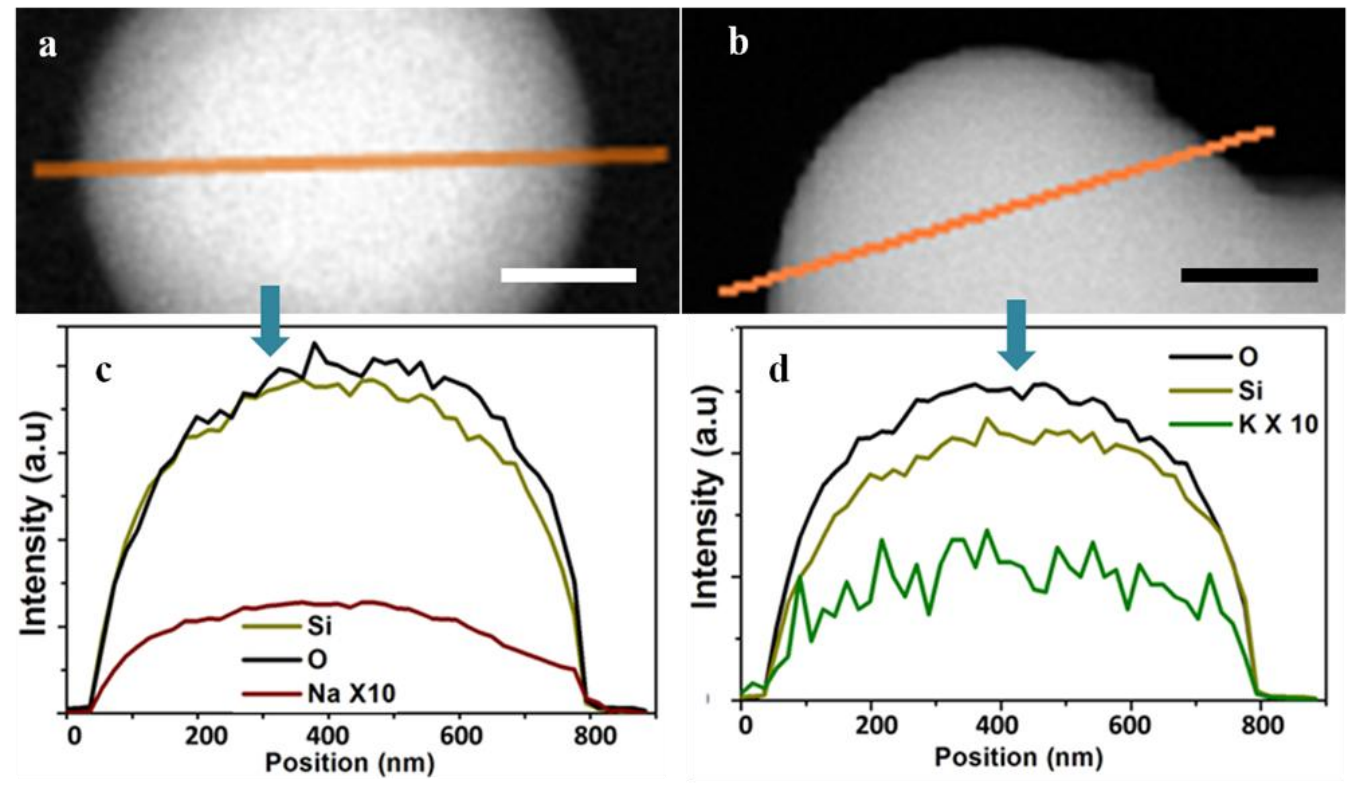

Figure 2.8. EDX line scan of (a) NaCl-doped silica nanoparticle, and (b) KCl-doped silica nanoparticle. Scale bar: $50 \mathrm{~nm}$. This is the same sample as shown in Figure 2.7f.

To distinguish these possibilities, prolonged vacuum treatment was used to cause the evaporation of the trapped $\mathrm{NH}_{3}$ and solvent. More specifically, the samples of Stöber silica were purified for 3 times and then dried in oven for $6 \mathrm{~h}$, before they were subjected to vacuum treatment. As shown by the elemental analysis in Figure $2.7 \mathrm{~b}$, the $\mathrm{C} / \mathrm{Si}$ molar ratio decreased dramatically from the $2.2 \%$ of the sample without vacuum to the $0.80 \%$ of the sample after $6 \mathrm{~h}$ vacuum, suggesting that the trapped solvent was the main source of $\mathrm{C}$. The N/Si molar ratio also decreased from $2.3 \%$ to the $1.0 \%$ after $6 \mathrm{~h}$ vacuum, indicating the loss of $\mathrm{NH}_{4}{ }^{+} / \mathrm{NH}_{3}$. 
To provide a direct comparison, TMAOH was used to replace $\mathrm{NH}_{3}$ in the silica synthesis to dramatically increase the $\mathrm{C}$ content in the doped silica. The samples were similarly purified and dried in oven for $6 \mathrm{~h}$. As shown by the elemental analysis (Figure 2.7c), the $\mathrm{C}$ content became much higher (13.5\%) and $\mathrm{N}$ content increased moderately to $3.8 \%$. These values did not show noticeable changes after vacuum for $6 \mathrm{~h}$, suggesting that the $\left(\mathrm{CH}_{3}\right)_{4} \mathrm{~N}^{+}$ions were tightly bound, likely because of their large size and the strong electrostatic interactions. The $\left(\mathrm{CH}_{3}\right)_{4} \mathrm{~N}^{+}$ion cannot dissociate, unlike $\mathrm{NH}_{4}{ }^{+}$which can convert to $\mathrm{H}^{+}$and gaseous $\mathrm{NH}_{3}$.

We compared the elemental analysis result before and after etching the silica nanoparticles in water. As shown in Figure $2.7 \mathrm{~d}$, the N/Si ratio of the ammonia-catalyzed silica decreased dramatically from $2.3 \%$ to $0.4 \%$, consistent with our expectations. It appeared that the inner layers of silica nanoparticles contained most of the counter ions, which were lost during the etching. The outer shells were formed by crosslinking monomers and short oligomers. Thus, they contained less trapped counter ions and were more resistant to the etching. As a direct comparison, we used inductively coupled plasma mass spectrometry (ICP-MS) to characterize the $\mathrm{Na}$ content in the $\mathrm{NaOH}$-catalyzed silica samples. As shown in Figure 2.7e, the Na/Si molar ratio decreased from the initial 11.2\% to the $0.4 \%$ after the etching, proving the presence of trapped $\mathrm{Na}^{+}$ions and supporting our hypothesis of inhomogeneous composition. 
Using $\mathrm{NH}_{3}$ as the base, we added $\mathrm{NaCl}, \mathrm{KCl}$, or $\mathrm{CsCl}(2 \mathrm{mM})$ in the synthesis, to investigate whether only the base or any counter ions can get trapped. As shown in the ICP-MS results in Figure 2.7f, the Na, K, Cs contents were consistently high at round $11-13 \%$. In particular, Cs is a rare impurity in the common chemicals. Its presence in silica with a high content gave unambiguous proof for the trapped ions. Energy-dispersive X-ray spectroscopy (EDX) line scan further demonstrated the presence of the metal ions inside the silica nanoparticles (Figure 2.7g-1), as opposed to mere surface adsorption.

\subsubsection{Synthesis of Stöber silica under different salt present}

$\mathrm{NaCl}, \mathrm{KCl}$ and $\mathrm{CsCl}$ were introduced in the Stöber synthesis (Section 2.1). Stock solutions of $100 \mathrm{mM}$ were prepared and aliquots were added to the $\mathrm{NH}_{3}$ solution in water/isopropanol, before the addition of TEOS, so that the final concentration was $2 \mathrm{mM}$. All other conditions were kept the same.

The EDX line scan data for $\mathrm{K}^{+}$and $\mathrm{Na}^{+}$doped silica nanoparticles have been added as Figure 2.8. EDX elemental mappings of aggregated silica nanoparticles (doped with $\mathrm{Au}$ and Pd) have been provided in the Figure 2.8, and Figure 2.9. 

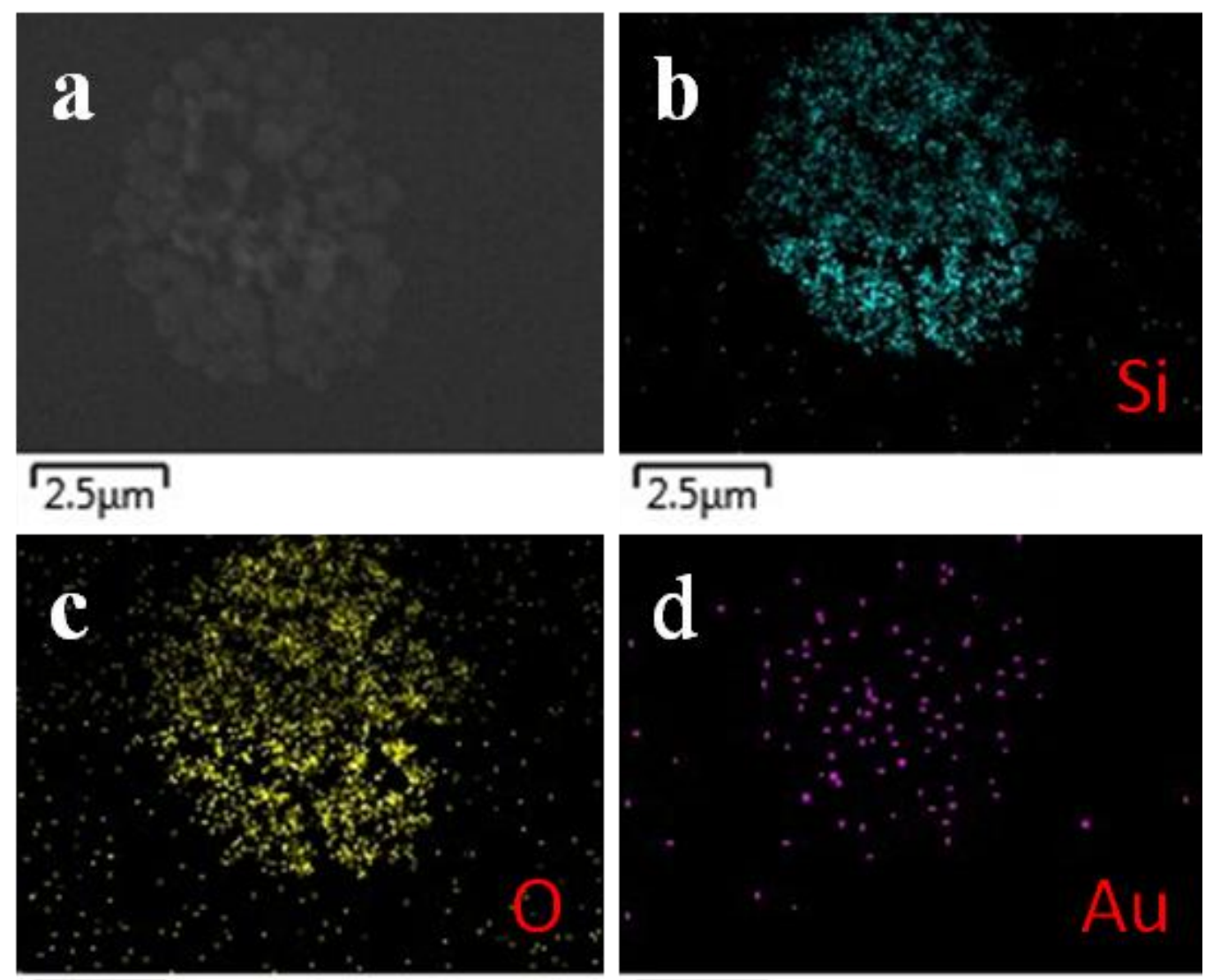

$\sqrt{2.5 \mu \mathrm{m}}$

$\sqrt{2.5 \mu \mathrm{m}}$

Figure 2.9. (a) SEM image of silica nanoparticles doped with $\mathrm{HAuCl}_{4}$, and $\mathrm{EDX}$

elemental mapping of (b) Si, (c) O, and (d) Au for the sample.

The Figure 2.9 is the silica nanoparticles mapping images under the SEM beam.

Here it is clear to see that the different elemental present in the silica nanoparticles, which can help to prove the encapsulation of traditional metals inside silica nanoparticles. The Au elemental presentation is not as high as $\mathrm{O}$, and Si inside silica shell. The reason is that Au ions ions paired with the polysilicic acid are not more than $\mathrm{Si}$, and $\mathrm{O}$ present inside the silica nanoparticles which is consistent with our hypothesis in the Chapter 2, ion-doping behavior inside silica nanoparticles are limited by solvent ratio, ion concentration, and so on. 

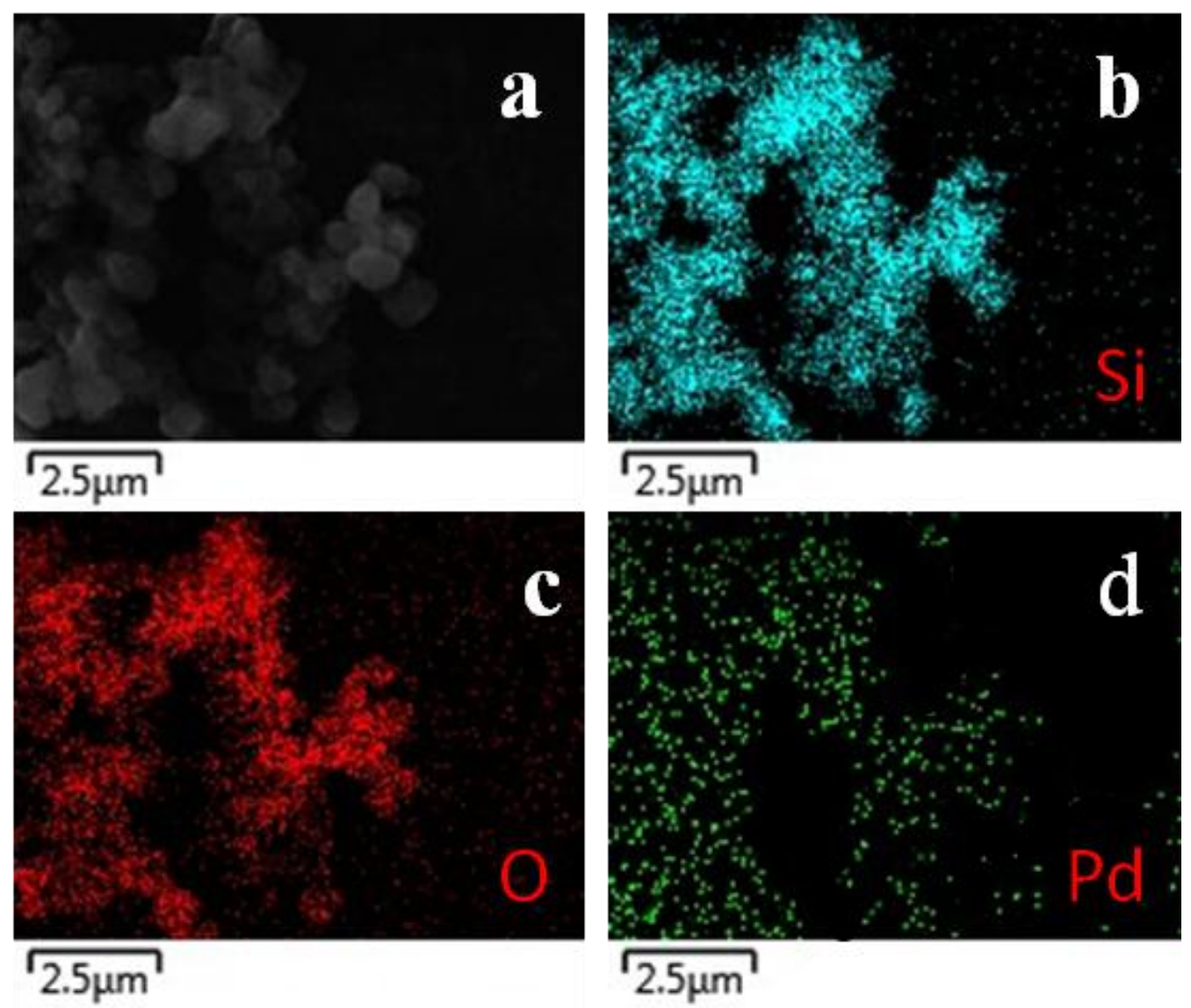

Figure 2.10. (a) SEM image of silica nanoparticles doped with $\mathrm{H}_{2} \mathrm{PdCl}_{4}$, and EDX elemental mapping of (b) Si, (c) O, and (d) Pd for the sample.

According to figure 2.10 , it is clear to see that $\mathrm{Pd}$ was doped inside silica nanoparticle. What we did is similar as Pt doping procedure: Pd precursor was added into Stöber silica preparation solution while adjusting to salt concentration is $2 \mathrm{mM}$ finally. Then the final product was centrifuged and purified 3 times before dried to do EDX mapping. Figure 2.9a is the SEM image of as synthesized silica nanoparticles, and figure 2.9b-d is the EDX mapping of the Pd doped silica: it is clear to see according to the EDX mapping that Pd was successfully doped inside silica nanoparticles when the mapping of Pd in figure $2.9 \mathrm{~d}$ is similar like it 
in figure $2.10 \mathrm{~b}$ while only mapping area smaller than that of figure $2.10 \mathrm{~b}$, and figure 2.10c, which means that the Pd content doped inside silica is lower than that of $\mathrm{Si}$, and $\mathrm{O}$.

\subsection{Conclusion}

The conventional views of silica do not discriminate its environment of formation, treating it as a single type of material with common characteristics. In this work, we demonstrated that the composition of Stöber silica varied greatly under different synthetic conditions. In particular, ion pairing played critical roles in the formation and disintegration of the silica framework. We provided evidence that the hollowing of silica nanoparticles originated not from surface redeposition or Ostwald ripening, but from the compositional inhomogeneity in terms of ion doping. The silica nanoparticles were highly swollen by the polar solvent, which was the origin of the microporosity after drying. 


\subsection{Bibliography}

(1) R. 121 Ghosh Chaudhuri, S. Paria, Chem. Rev. 2011, 112, 2373-2433.

(2) J. 122Lee, Y. Lee, J. K. Youn, H. B. Na, T. Yu, H. Kim, S.-M. Lee, Y.-M. Koo,

J. H. Kwak, H. G. Park, H. N. Chang, M. Hwang, J.-G. Park, J. Kim, T. Hyeon, Small 2008, 4, 143-152.

(3) F. G. 123Aliev, M. A. Correa-Duarte, A. Mamedov, J. W. Ostrander, M. Giersig, L. M. Liz-Marzán, N. A. Kotov, Adv. Mater. 1999, 11, 1006-1010.

(4) Purkayastha, A.; Baruah, J. B. Appl. Organomet. Chem. 2004, 18, 166.

(5) Despas, C.; Walcarius, A.; Bessière, J. Talanta 1997, 45, 357.

(6) Green, M.; Harries, J.; Wakefield, G.; Taylor, R. J. Am. Chem. Soc. 2005, 127, 12812.

(7) Kobayashi, Y.; Misawa, K.; Kobayashi, M.; Takeda, M.; Konno, M.; Satake,

M.; Kawazoe, Y.; Ohuchi, N.; Kasuya, A. Colloids Surf., A 2004, 242, 47.

(8) Sadasivan, S.; Fowler, C. E.; Khushalani, D.; Mann, S. Angew. Chem. Int. Ed. 2002, $41,2151$.

(9) Alexander, G. B.; Heston, W. M.; Iler, R. K. J. Phys. Chem. 1954, 58, 453.

(10) Slowing, I. I.; Trewyn, B. G.; Giri, S.; Lin, V. S. Y. Adv. Funct. Mater. 2007, $17,1225$.

(11) Bae, S. W.; Tan, W.; Hong, J.-I. Chem. Commun. 2012, 48, 2270.

(12) Sartbaeva, A.; Haines, J.; Cambon, O.; Santoro, M.; Gorelli, F.; Levelut, C.;

Garbarino, G.; Wells, S. A. Phys. Rev. B 2012, 85, 064109. 
(13) Ma, K.; Sai, H.; Wiesner, U. J. Am. Chem. Soc. 2012, 134, 13180.

(14) Slowing, I. I.; Vivero-Escoto, J. L.; Trewyn, B. G.; Lin, V. S. Y. J. Mater. Chem. 2010, 20, 7924.

(15) Kresge, C. T.; Leonowicz, M. E.; Roth, W. J.; Vartuli, J. C.; Beck, J. S. Nature 1992, 359, 710; Sartbaeva, A.; Wells, S. A.; Treacy, M. M. J.; Thorpe, M. F. Nat Mater 2006, 5, 962; Fang, X.; Zhao, X.; Fang, W.; Chen, C.; Zheng, N. Nanoscale 2013, 5, 2205; Zhang, Q.; Zhang, T.; Ge, J.; Yin, Y. Nano Lett. 2008, 8, 2867.

(16) Lou, X. W.; Wang, Y.; Yuan, C.; Lee, J. Y.; Archer, L. A. Adv. Mater. 2006, 18, 2325 .

(17) Zhang, Q.; Wang, W.; Goebl, J.; Yin, Y. Nano Today 2009, 4, 494.

(18) Dove, P. M.; Han, N.; Wallace, A. F.; De Yoreo, J. J. Proc. Natl. Acad. Sci. 2008, 105, 9903.

(19) Lou, X. W.; Archer, L. A.; Yang, Z. Adv. Mater. 2008, 20, 3987.

(20) Cheng, K.; Sun, S. Nano Today 2010, 5, 183.

(21) Yec, C. C.; Zeng, H. C. J. Mater. Chem. A 2014, 2, 4843.

(22) Roca, M.; Haes, A. J. J. Am. Chem. Soc. 2008, 130, 14273.

(23) Du, J.; Qi, J.; Wang, D.; Tang, Z. Energy Environ. Sci. 2012, 5, 6914.

(24) Dong, Z.; Lai, X.; Halpert, J. E.; Yang, N.; Yi, L.; Zhai, J.; Wang, D.; Tang, Z.; Jiang, L. Adv. Mater. 2012, 24, 1046.

(25) Wong, Y. J.; Zhu, L.; Teo, W. S.; Tan, Y. W.; Yang, Y.; Wang, C.; Chen, H. J. 
Am. Chem. Soc. 2011, 133, 11422.

(26) Li, H.; Xin, H. L.; Muller, D. A.; Estroff, L. A. Science 2009, 326, 1244.

(27) Estroff, L. A.; Cohen, I. Nat Mater 2011, 10, 810.

(28) Song X. H., Ding T., Chen H. Y., Small 2015, 11, 4351-4365. 


\section{Chapter 3 Exploring the Ion Pairing Formation Mechanism into Application}

\subsection{Introduction}

It is well known that silica can be synthesized by solution methods and such silica has been widely used in industry and biomedical research. But misconceptions are common with silica. As such, it is difficult to preempt problems and to design for new properties. Many people (at least those I have talked to) think that silica is ONE type of material with common characteristics. For example, silica shells should be highly crosslinked, insoluble in water, and to a large extent impermeable, just like the glass in our windows. But these are not true.

Silica is an old material but misconceptions are abundant with regard to silica nanoparticles. Here, we investigate the fundamental reasons for their hollowing upon etching. We realize that, during the synthesis, the silica precursors are essentially ion-paired polyelectrolytes, whose nucleation depends on the concentration of the counter ions, the solvent polarity, and most importantly, the size/length of the poly(silicic acid). Thus, the "silica" that nucleates out at the different stages of synthesis has different degrees of ion doping, which explains its unusual solubility in water, its microporosity, and the selective hollowing phenomena. Our new view with the ionic impurities on the central stage allows 
for insights in silica properties and versatility in synthetic design..

We believe that the understanding of the inner workings of silica is essential for its applications. As proof-of-concept, we demonstrated the doping of coordination complexes and an anti-cancer drug (Cisplatin) in silica. Furthermore, the silica porosity can be rationally controlled by doping organics and by acid treatment. We are currently working on the hollowing phenomena of other types of oxides, as the underlying principles should be common.

\subsection{Materials and methods}

\subsubsection{Materials}

Materials and Characterization Methods. All solutions were prepared using deionized water (resistivity $\left.>18 \mathrm{M} \bullet \mathrm{cm}^{-1}\right)$. 4-mercaptobenzoic acid (MBA, 90\%), 2-naphthalenethiol (99\%), 1-naphthalenethiol (99\%,), biphenyl-4-thiol (BP4T) (97\%), 2-phenylethanethiol (2-PET) (98\%), butanethiol (BuSH) (99\%), 1-decanthiol (DoSH) (99\%), 1-dodecanethiol (DodSH) (99\%), 1-octadecanthiol (OctdSH) (99\%), 3-mercaptopropanoic acid (3-MPA)(99\%), 16-mercaptohexadecanoic acid (16-MHDA) (99\%), 3-aminopropyltriethoxysilane (APTES, 98\%), sodium citrate tribasic dihydrate (99.0\%), L-ascorbic acid (99\%) were purchased from Sigma Aldrich and used as received. Hydrogen tetrachloroaurate(III) (HAuCl4, 99.9\%, Au 49\% on metals basis, Alfa Aesar), 4-ethylthiophenol (4-ETP)(97\% Alfa Aesar), sodium borohydride, and ethanol (analytical grade) were used as received. Copper 
specimen grids (200 mesh) with formvar/carbon support film (referred to as TEM grids in the text) were purchased from Beijing XXBR Technology Co..

Field emission scanning electron microscopy (SEM) images were collected on a JEOL JSM-6700F. Transmission electron microscopy (TEM) images were collected on a JEM-1400 (JEOL) operated at $100 \mathrm{kV}$.

SERS spectra were collected directly from the substrate on a PeakSeeker Pro spectrometer (Raman Systems Inc.) using a red laser $(\lambda=785 \mathrm{~nm})$ at $290 \mathrm{~mW}$.

\subsubsection{Methods}

Synthesis of Au seeds $5 \mathrm{~nm}$ AuNP seeds are synthesized based on the reported method, ${ }^{1}$ basically, $1.47 \mathrm{mg}$ of sodium citrate and $1.97 \mathrm{mg}$ of $\mathrm{HAuCl}_{4} \cdot \mathrm{xH}_{2} \mathrm{O}$ were dissolved in $20 \mathrm{~mL}$ water. Then, $0.6 \mathrm{~mL}$ of ice-cold $\mathrm{NaBH} 4$ solution $(0.1 \mathrm{M})$ was added with vigorous stirring. The solution turned from orange-yellow to brownish-red, indicating the formation of AuNP. The seed solution could be used directly after 2 hours.

$60 \mathrm{~nm} \mathrm{Au}$ NPs are synthesized based on the reported method. ${ }^{2}$ Citrate-stabilized AuNP with a diameter of $40 \mathrm{~nm}$ was first synthesized by reducing boiling $100 \mathrm{~mL} \mathrm{HAuCl} 4 \cdot \mathrm{xH}_{2} \mathrm{O}(0.1 \mathrm{mg} / \mathrm{ml})$ with $0.75 \mathrm{~mL}$ sodium citrate solution ( $1 \% \mathrm{wt}$ ), followed by 11 cycles of addition of extra $\mathrm{HAuCl}_{4} \cdot \mathrm{xH}_{2} \mathrm{O}$, sodium citrate and $\mathrm{NaOH}$ solution as well as boiling water.

$20 \mu \mathrm{L}$ 11-MUA ( $2 \mathrm{mM}$ in ethanol) was added. After $5 \mathrm{~min}, 600 \mu \mathrm{L}$ TEOS (8.96 $\mathrm{mM}$ in water) was added, followed by $90 \mu \mathrm{L}$ of ammonia $(25-28 \% \mathrm{w} / \mathrm{w})$. 
Concentric core-shell AuNP@silica were obtained after $12 \mathrm{~h}$ of reaction at room temperature. To isolate the AuNP@silica, the reaction mixture was centrifuged at $5200 \mathrm{~g}$ for $9 \mathrm{~min}$ and the supernatant removed. The residual NPs were re-dispersed in ethanol.

The AuNP@silica as synthesized were functionalized with amino group by APTES.AuNP@silica solution $(1.5 \mathrm{~mL})$ was concentrated to $\sim 6 \mu \mathrm{L}$ by centrifugation at $5200 \mathrm{~g}$ for $9 \mathrm{~min}$. After the removal of the supernatant, the isolated NPs were added into a solution containing $500 \mu \mathrm{L} \mathrm{H}_{2} \mathrm{O}, 500 \mu \mathrm{L}$ ethanol and APTES (1 mM). Amino-functionalized AuNP@Silica were obtained after 1 $\mathrm{h}$ reaction with vigorous stirring. The NPs were purified twice by centrifugation in ethanol at $5200 \mathrm{~g}$ for $9 \mathrm{~min}$. Consequently, the concentrated NPs were added into the Au seeds solution $(500 \mu \mathrm{L})$ and incubated for $2 \mathrm{~h}$

\subsection{Results and discussion}

Seeds are usually the citrate-stabilized Au nanoparticles of 3-5 $\mathrm{nm}$ in diameter.

Then, the seed-decorated substrate was immersed in the reactant solution containing the ligand 4-MBA $(0.55 \mathrm{mM})$, the Au precursor $\mathrm{HAuCl}_{4}(1.7 \mathrm{mM})$, and the reducing agent L-ascorbic acid $(4.1 \mathrm{mM})$. After $15 \mathrm{~min}$, the substrate was retrieved, rinsed, and dried in air. Typically, a dense forest of ultrathin Au nanowires $(\mathrm{d}=6 \mathrm{~nm}, 1=1 \mu \mathrm{m})$ would form on the substrate surface. In the following, the reaction parameters are systematically varied to study the generality of the Active Surface Growth mode. 


\subsubsection{Rational synthesis of silica nanostructures}

The Stöber silica discussed above was synthesized in water/isopropanol $=2: 5$ mixture solutions. Varying the solvent ratio is expected to affect the solubility of the ion-paired poly(silicic acid) and thus, the shell thickness after hollowing. More specifically, higher water content means more polar solvent, allowing longer poly(silicic acid) to remains solvated in the solution. Thus, there are more silica precursors left over for the $2^{\text {nd }}$ stage growth. The experimental observations were fully consistent with this expectation: When the water content was increased (to solvent ratio of 3:5), the resulting silica shell after etching increased from 8 to $12 \mathrm{~nm}$; when the water content was decreased (to 1:5), the shells became much thinner $(4 \mathrm{~nm})$ and the interior was more difficult to etch (Figure 3.1b, 3.1f); when the water content was further decreased (to 1:20), there was no recognizable outer shell after the etching (Figure 3.1a, 3.1e). At this extreme condition, nearly all silica precursors have nucleated out, leaving barely anything for the $2^{\text {nd }}$ stage growth. The less polar solvent also led to a lower degree of ion pairing and thus, a higher degree of silica crosslinking, making the resulting silica more difficult to etch. ${ }^{3,4}$ We screened the etching conditions for this sample (as shown in Figure 3.1e), hollow structure was never obtained suggesting that these silica nanoparticles were more uniform in composition. 

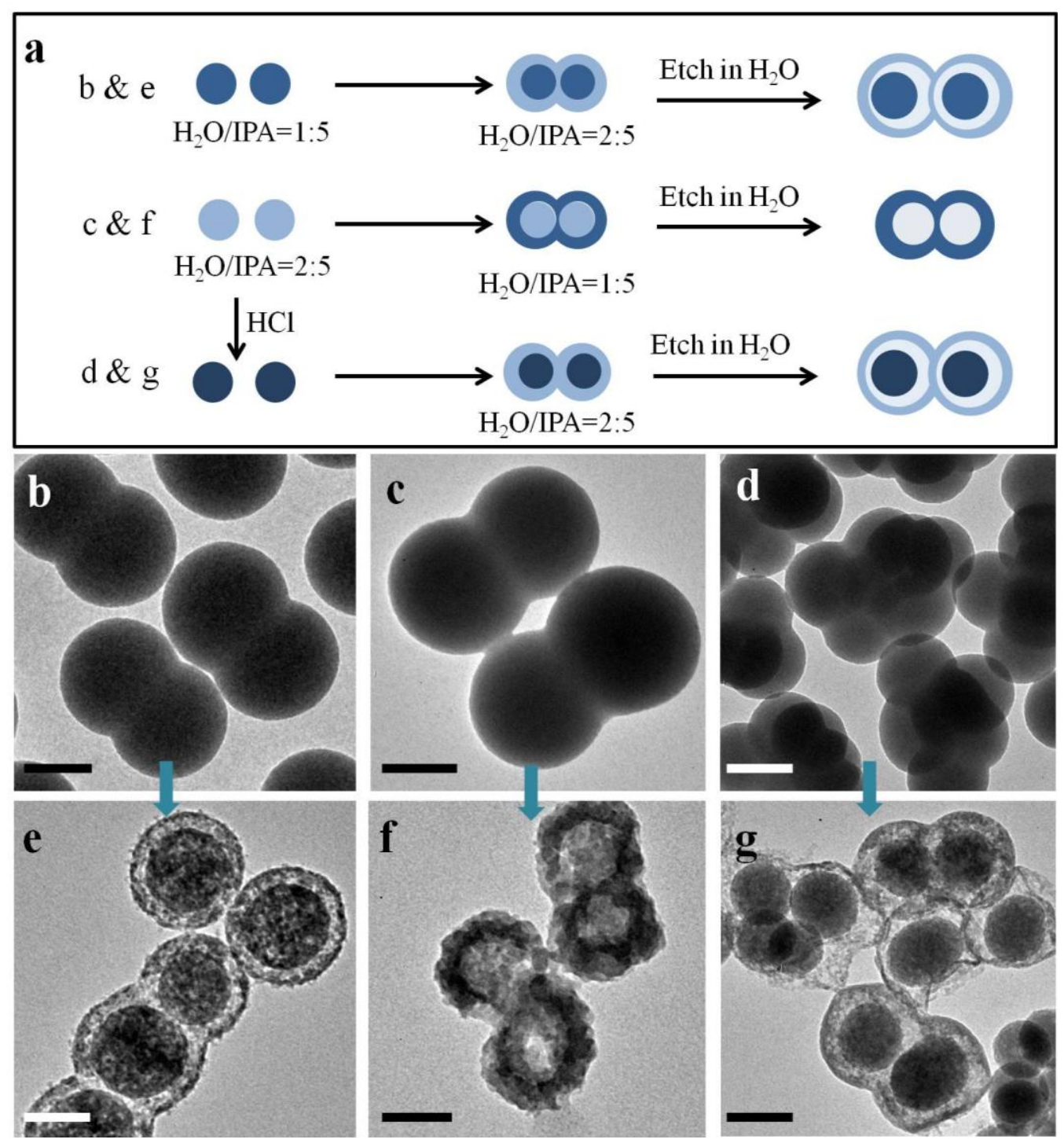

Figure 3.1. (a) Schematics illustrating the synthetic designs. The resulting nanoparticles have similar appearance initially (b-d) but were very different (e-g, respectively) after the etching in $90{ }^{\circ} \mathrm{C}$ water. (b) Silica nanoparticles were first synthesized in water/isopropanol $=1: 5$ solution and then $2^{\text {nd }}$ step growth in 2:5 solution; (c) silica nanoparticles were synthesized in 2:5 first and then in 1:5 solution; (d) the silica nanoparticles synthesized in 2:5 solution were treated in 2 $\mathrm{M} \mathrm{HCl}$, purified, and then subjected to $2^{\text {nd }}$ step growth in 2:5 solution. Scale bar: $50 \mathrm{~nm}$. Reprinted with permission from ref. [66], respectively, copyright 2015 


\section{Wiley-VCH.}

With the above understanding, we can further design silica nanostructure by controlling solvent ratio, salt concentration, and the time length of acid treatment. For a proof-of-concept, we designed two-step reactions with different conditions as shown in Figure 3.1: 1) when the $1^{\text {st }}$ step used low water content solvent and the $2^{\text {nd }}$ step used high water content, the inner layer was more difficult to etch than the outer layer. Thus, after the $2^{\text {nd }}$ layer turned hollow upon etching, the inner layer was hollow but to a lower extent (Figure 3.1b,e). 2) When the $1^{\text {st }}$ step used high water content and the $2^{\text {nd }}$ step low water content, the outer layer remained after etching while the inner layer turned hollow (Figure 3.1c,f). 3) The silica nanoparticles from the $1^{\text {st }}$ step synthesis was treated by acid, and purified, before being used as seeds for the $2^{\text {nd }}$ step growth of normal silica. The $\mathrm{H}^{+}$ions can exchange with the trapped $\mathrm{NH}_{4}{ }^{+}$ions, allowing crosslinking of the resulting -SiOH groups (Figure 3.2b). After etching, the outer layer became hollow while the acid-treated section remained intact (Figure 3.1d,g). These simultaneous etching experiments in the same solution provide strong support for the relative easiness in etching; the results are fully consistent with our hypothesis. Importantly, our abilities to design the etchable sections open windows for new types of synthesis in the future. ${ }^{5,6}$

\subsubsection{Doping heavy metals inside silica}

Complexes such as $\left[\mathrm{Cu}\left(\mathrm{NH}_{3}\right)_{\mathrm{x}}(\mathrm{OH})_{\mathrm{y}}\right]^{2+}(\mathrm{x}+\mathrm{y}=6)$ carry positive charges and 
contain H-bonding groups $\left(-\mathrm{NH}_{3}\right.$ and $\left.-\mathrm{OH}_{2}\right)$, allowing them to interact with $-\mathrm{SiOH}$ and negatively charged ions. We add $\mathrm{Cu}^{2+}(1.35 \mathrm{mM})$ to a normal Stöber synthesis $\left(0.47 \mathrm{M} \mathrm{NH}\right.$ ), generating $\left[\mathrm{Cu}\left(\mathrm{NH}_{3}\right)_{\mathrm{x}}(\mathrm{OH})_{\mathrm{y}}\right]^{2+}$ in situ before the reaction. After 3 cycles of purification, the resulting silica appeared blue in color (Figure 3.2d), vividly indicating the presence of embedded $\mathrm{Cu}$ ions. EDX scan confirmed the presence of $\mathrm{Cu}$ in the sample (Figure 3.2e). In TEM images (Figure 3.2a), these doped silica nanoparticles showed a clean boundary between the $\mathrm{Cu}$-doped darker contrast layer and the lighter outmost layer, consistent with the proposed two-stage growth. The inner layer can be readily etched by heating in neutral water (Figure 3.2b). When $\mathrm{NH}_{2} \mathrm{NH}_{2}$ was added to reduce the embedded $\mathrm{Cu}(\mathrm{II})$ ions, metallic $\mathrm{Cu}$ formed at the nanoparticle core, which also became hollow because of the basic solution (Figure 3.2c). The formation of metallic $\mathrm{Cu}$ nanoparticles at the core and their absence in the shell clearly indicated that most of the complex ions were doped in the core, supporting the two-stage nucleation.

Using Au@silica as the seeds, we can grow doped silica on their surface. $\mathrm{H}_{2} \mathrm{PtCl}_{6}, \mathrm{HAuCl}_{4}, \mathrm{H}_{2} \mathrm{PdCl}_{4}$, and Cisplatin (cis- $\left[\mathrm{Pt}\left(\mathrm{NH}_{3}\right)_{2} \mathrm{Cl}_{2}\right]$ ) were directly added to the Stöber synthesis. In the resulting nanoparticles (Figure 3.2e-h), it can be observed that the heavy metal ions were embedded, giving a dark contrast The doping was not as extensive as the $\mathrm{Cu}$ complexes, likely due to the slow ligand exchange of $\mathrm{Cl}^{-}$by ammonia $\mathrm{NH}_{3}$. layer sandwiched between the initial Au@silica seed and the outmost silica layer. EDX verified the presence of these 
noble metal ions in the silica nanoparticles. ${ }^{3}$
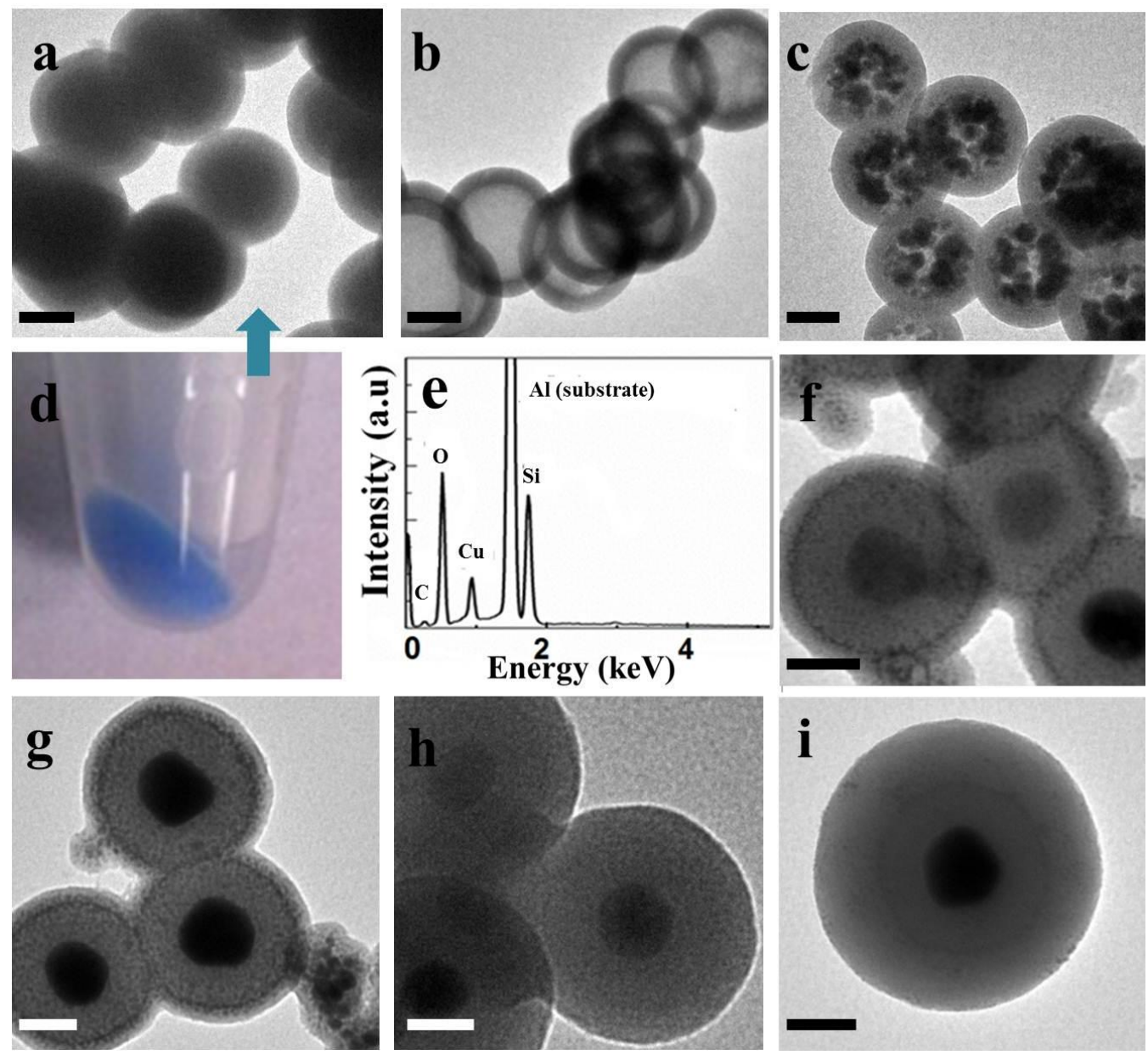

Figure 3.2. Doping transition metal ions in silica. (a-e) Silica doped with $\left[\mathrm{Cu}\left(\mathrm{NH}_{3}\right)_{\mathrm{x}}\left(\mathrm{OH}_{2}\right)_{\mathrm{y}}\right]^{2+}$ ions (a) before and (b) after etching; (c) after reduction to $\mathrm{Cu}$ nanoparticles using $\mathrm{NH}_{2} \mathrm{NH}_{2}$; (d) photograph of sample a; (e) EDX measurement of sample a that was dried on an Al substrate. (f-i) Using Au@ silica as seeds, the silica shells were doped with noble metal ions by adding (f) $\mathrm{H}_{2} \mathrm{PtCl}_{6}$; (g) $\mathrm{HAuCl}_{4}$; (h) $\mathrm{H}_{2} \mathrm{PdCl}_{4}$; (i ) Cisplatin. Scale bar: $50 \mathrm{~nm}$. Reprinted with permission from ref. [66], respectively, copyright 2015 Wiley-VCH. 
(a)

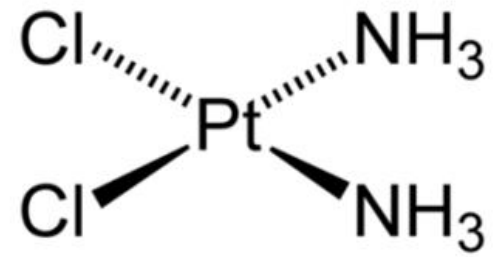

Cisplatin (b)

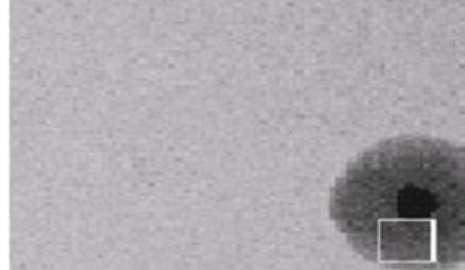

SE2 20II Dnm

(d)

\begin{tabular}{|l|l|l|}
\hline Element & Wt $\%$ & At\% \\
\hline OK & 31.69 & 52.50 \\
\hline SiK & 39.12 & 36.92 \\
\hline CuK & 23.55 & 09.82 \\
\hline PtL & 05.64 & 00.77 \\
\hline Matrix & Correction & MThin \\
\hline
\end{tabular}

Figure 3.3. Incorporating Cisplatin in Au@silica nanoparticles (a) molecular formula of Cisplatin; (b) the SEM image of the Au@silica; (c) EDX analysis of the framed region as shown in b; and (d) list of the elements as shown in c. This is the same sample as used in figure 3.2

\subsubsection{Doping organic molecules and drugs inside silica}

For doping organic dyes, positively charged Methylene Blue and negatively charged carboxyfluorecesin were added as solid into the ammonia solution (1.44 $\mathrm{mL}$, same as above). After the dyes are fully dissolved (final concentration of 2 $\mathrm{mM}), 2 \mu \mathrm{L}$ of neat TEOS was added to initiate the silica formation. To avoid surface adsorption of the organic dyes, extra purification cycles were applied. All other conditions were kept the same. 

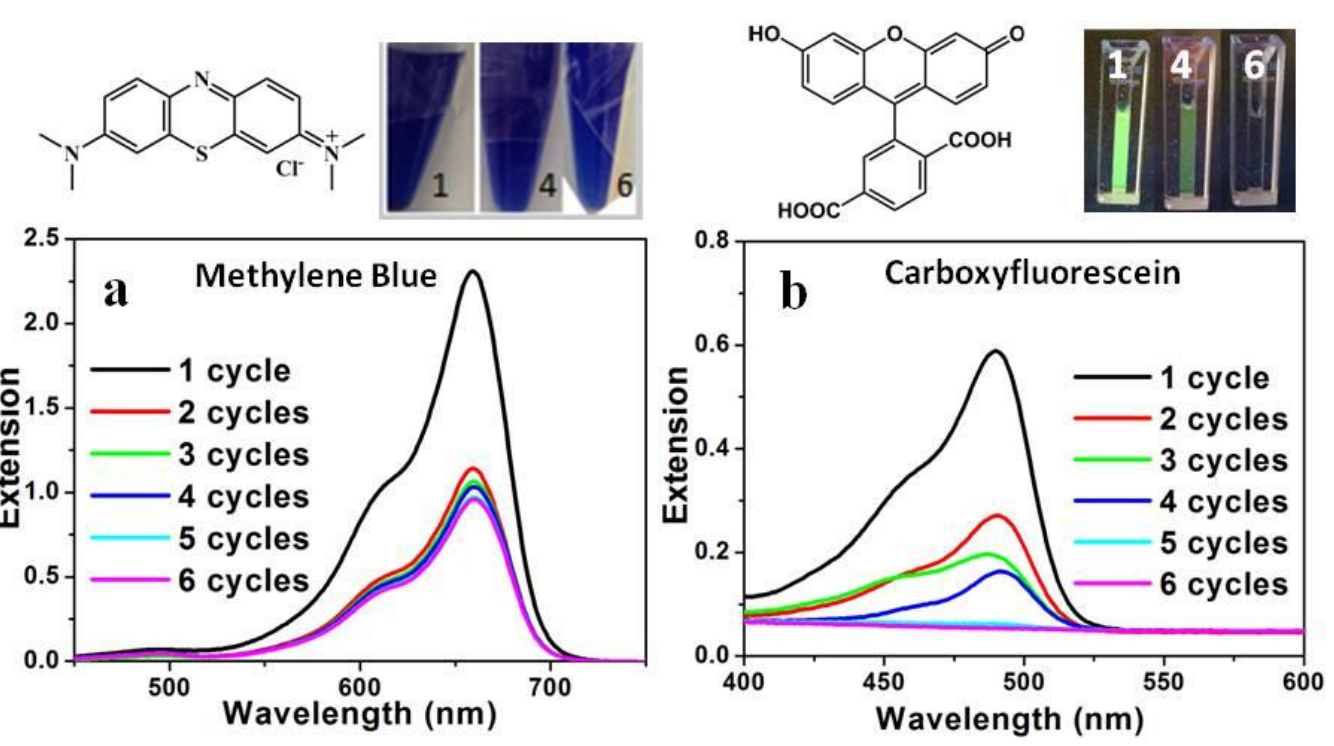

Figure 3.4. Doping ammonia-catalyzed silica with organic dyes: (a) positively charged Methylene Blue; (b) negatively charged carboxyfluorescein. The samples were purified in water for 6 cycles and the change of UV-Vis spectra was recorded. Reprinted with permission from ref. [66], respectively, copyright 2015 Wiley-VCH.

Similarly, we can dope positively charged organic molecules or drugs in silica. Fluorescent dyes were used as a proof-of-concept and a model for positively charged drug molecules. For clear contrast, positively charged 3,7-bis(dimethylamino)-phenothiazin-5-ium chloride (Methylene Blue) and negatively charged carboxyfluorescein were selected. They were directly added to the Stöber synthesis. The incorporated dyes in the resulting nanoparticles can be tracked by the absorption and fluorescence of the solution. For Methylene Blue, the blue color remained with the isolated silica nanoparticles, even after 6 cycles of purification (figure 3.4a), indicating its strong interactions with silica. 
For carboxyfluorescein (figure 3.4b), it appeared that some molecules were incorporated in the silica. But the color quickly disappeared and the solution was almost colorless after 5 cycles of purification. Thus, the positively charged dye appeared to be more tightly bound than the negatively charge dye, consistent with our expectations.

As shown in figure 3.4 , the cancer drug cisplatin was used during the silica nanoparticle synthesis which was used as positive charged counter ions during polysilicic acid $1^{\text {st }}$ stage nucleation. It is clear to identify the encapsulation of cisplatin by EDX analysis in figure $3.3 \mathrm{c}-\mathrm{d}$. It is very important to future application in drug delivery according to this control experiments on drug encapsulation, and organic molecule encapsulation.

\subsubsection{Tuning the porosity of silica}

With the above knowledge, we can also modulate the amount of doped organics to control the porosity of silica. Different amounts of TMAOH were used as the catalyst during the silica synthesis. The resulting nanoparticles were purified, dried in oven for $6 \mathrm{~h}$, and then subjected to Brunauer-Emmett-Teller (BET) measurements (Figure 3.5a). The silica surface area increased with the increase of $\left(\mathrm{CH}_{3}\right)_{4} \mathrm{~N}^{+}$loading (Figure 3.5b), providing a facile means of modulation.

Acid treatment can reduce silica porosity. Briefly, Au@silica nanoparticles 

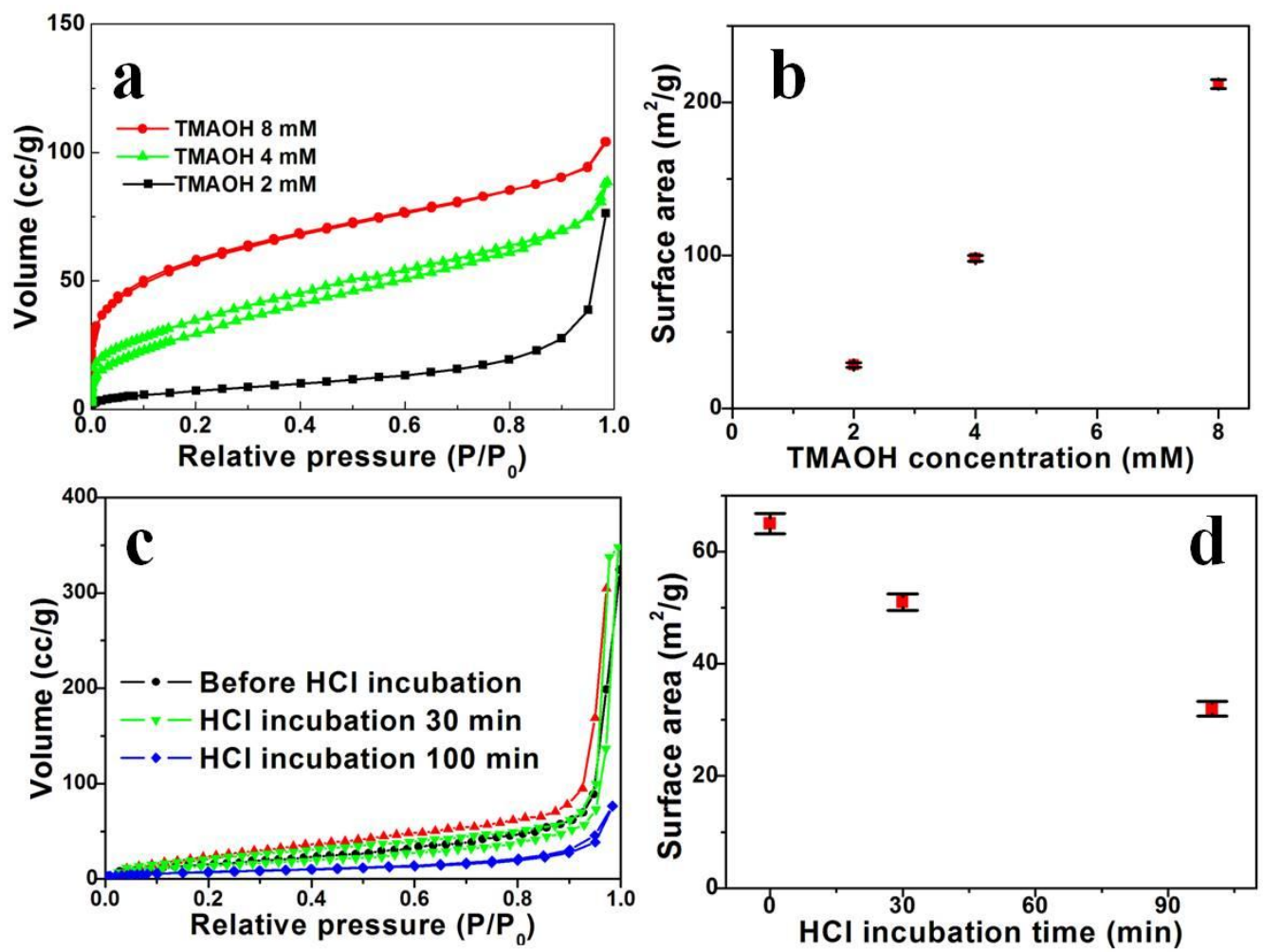

Figure 3.5. Tuning silica porosity by doping. (a) Nitrogen adsorption and desorption isotherms of the silica nanoparticles catalyzed using different concentrations of TMAOH. (b) The estimated surface areas of the samples in a. (c) Nitrogen adsorption and desorption isotherms of the silica nanoparticles after treatment in $\mathrm{HCl}(2 \mathrm{M})$ for 0,30 , and $100 \mathrm{~min}$. (d) The estimated surface area of the samples in c.

were purified, incubated in concentrated $\mathrm{HCl}$ solution $(2 \mathrm{M})$, and then purified for 3 cycles to completely remove the acid. The isolated Au@ silica nanoparticles were etched in $\mathrm{KCN}$ solution $(40 \mathrm{mM})$ for $24 \mathrm{~h}$ at room temperature (CAUTION: $\mathrm{KCN}$ is highly toxic and should NOT be added to acidic solutions). Without the acid treatment, a control sample gave hollow silica nanoparticles (Figure 3.6c), 

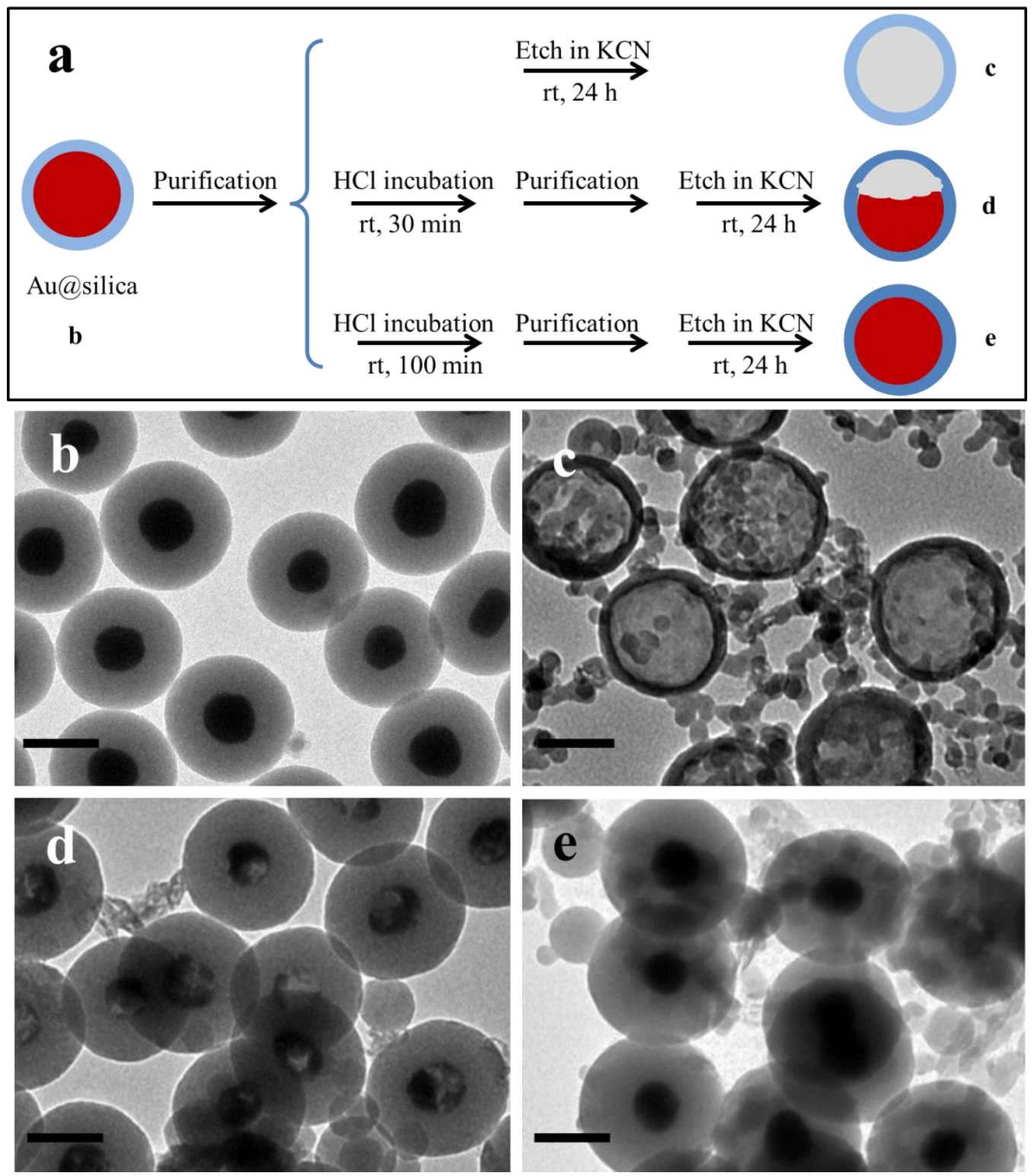

Figure 3.6. (a-e) Schematics and TEM images showing the modulation of silica

porosity by acid treatment. (b) Ammonia-catalyzed Au@silica nanoparticles after purification; (c) after etching sample b in $\mathrm{KCN}$; (d) sample b was treated in $2 \mathrm{M} \mathrm{HCl}$ for $30 \mathrm{~min}$, purified, and then etched in $\mathrm{KCN}$; (e) sample b was treated in $2 \mathrm{M} \mathrm{HCl}$ for $100 \mathrm{~min}$, purified, and then etched in KCN. Scale bar: $50 \mathrm{~nm}$. Reprinted with permission from ref. [66] respectively, copyright 2015 Wiley-VCH. 
where the $\mathrm{Au}$ was etched by $\mathrm{CN}^{-}$and part of the silica shell was etched by the slightly basic solution. In contrast, the sample with $30 \mathrm{~min}$ acid treatment gave
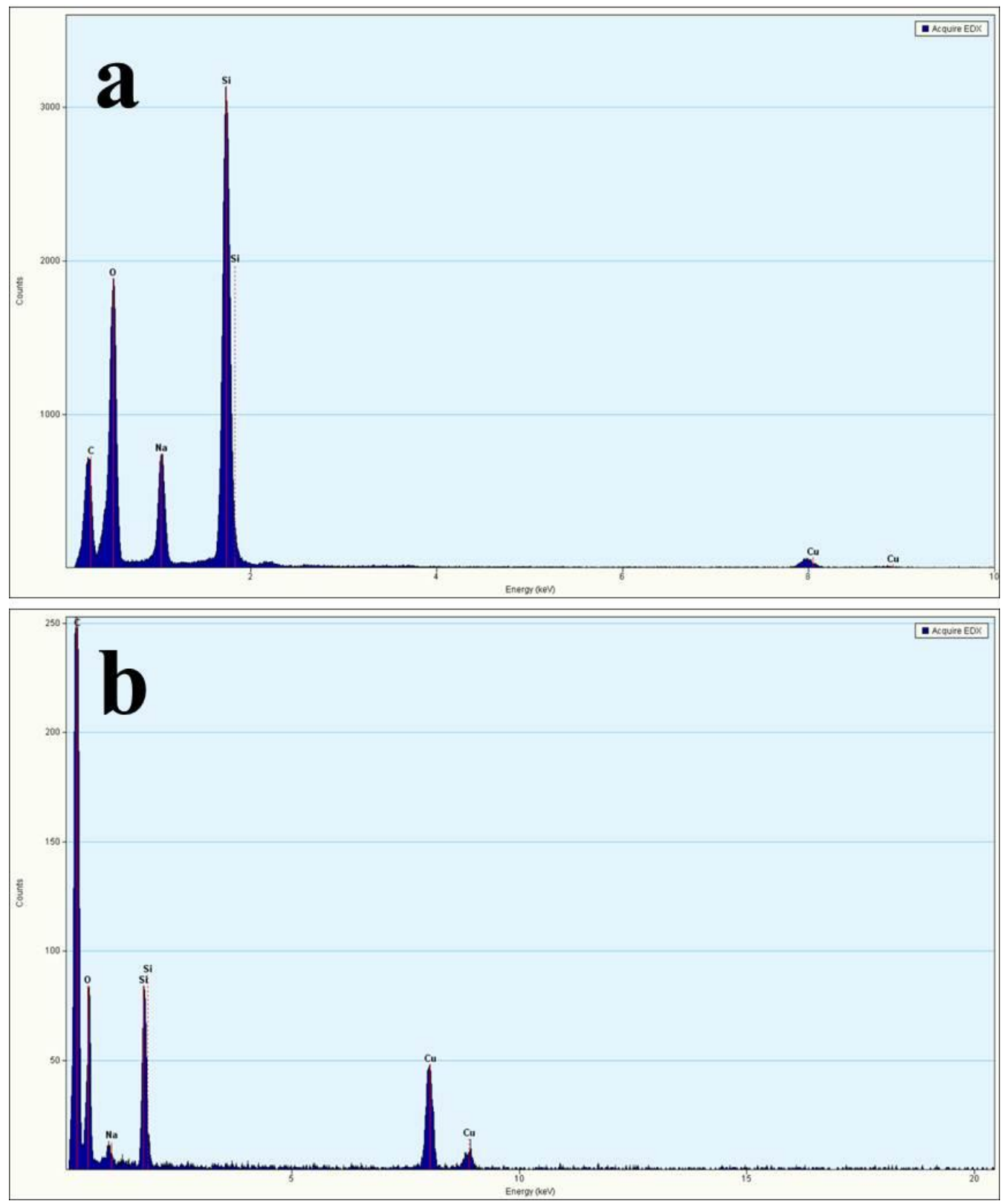

Figure 3.7. EDX area scan for $\mathrm{NaOH}$-catalyzed silica nanoparticles (a) before

$\mathrm{HCl}$ incubation, (b) after incubation in $\mathrm{HCl}$ solution (2 M) for $30 \mathrm{~min}$.

partially etched Au core whereas the silica domain remained largely unchanged

(Figure 3.6d). For the sample with 100 min acid treatment, neither the Au cores 
nor silica shells showed noticeable change (Figure 3.6e). Evidently, the acid treatment led to ion exchange, greatly reducing the silica porosity and suppressing the ionic diffusion $\left(\mathrm{CN}^{-},\left[\mathrm{Au}(\mathrm{CN})_{4}\right]^{-}, \mathrm{NH}_{4}^{+}\right.$, etc. $)$therein. EDX measurements of $\mathrm{NaOH}$-catalyzed silica nanoparticles showed significant decrease of $\mathrm{Na}$ content after the acid treatment (Figure 3.7), proving the ion exchange.

BET measurements were carried out to verify the change of porosity in silica nanoparticles (Figure 3.7c,d). Before acid treatment, the Stöber silica had small surface area of about $64.2 \mathrm{~m}^{2} / \mathrm{g}$. After it, the surface area decreased to $53.8 \mathrm{~m}^{2} / \mathrm{g}$ at $30 \mathrm{~min}$ and $32.2 \mathrm{~m}^{2} / \mathrm{g}$ at $100 \mathrm{~min}$ of acid treatment. The clear trend of decreasing porosity with the acid treatment is consistent with our proposed mechanism.

The micropores of silica play important roles in applications, for example, when catalysts or drug molecules are loaded in silica substrates. Unlike mesopores, ${ }^{7-9}$ the methods for tuning the microporosity of silica are not well developed and understood. Thus, we believe that our understanding of silica and the ability to tune its mircopores will lead to new synthetic designs for applications.

\subsubsection{Silica shell fusion control}

With the above understanding, it is possible to control the silica nanoparticles shell fusion by tuning the salt concentration, the addition time, mixed with the 
seed induced growth procedure, which can help us to get novel silica nanostructures. The tuning procedure can help to control the TEOS hydrolysis
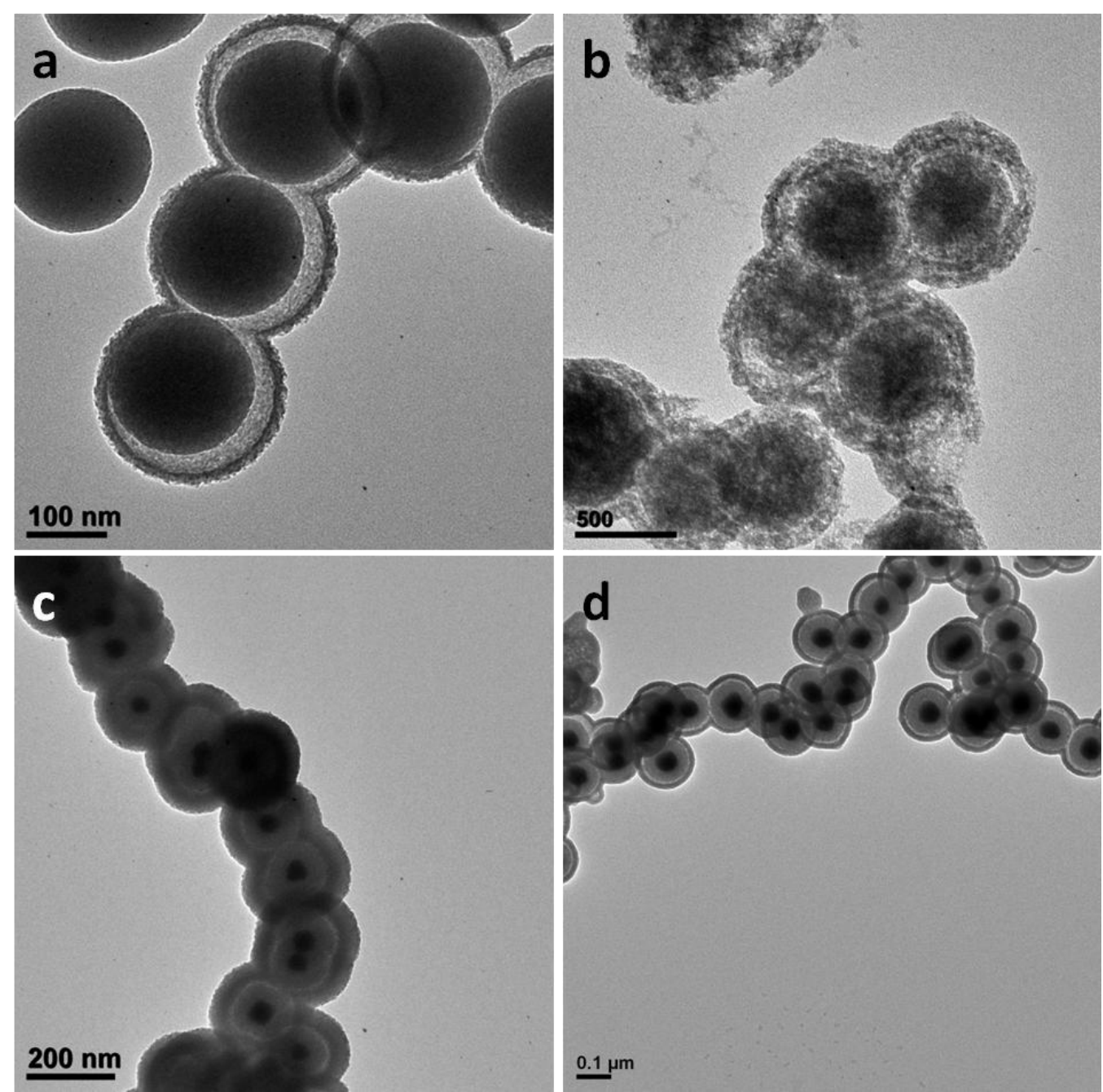

Figure 3.8. TEM images of fused silica nanoparticles under different control conditions (a) double layers silica nanoparticles; (b) Triple layers silica nanoparticles; (c) $\mathrm{Cu}^{2+}$ doped silica shell fusion $\left(\mathrm{Cu}^{2+}\right.$ added time was similar as TEOS); (d) $\mathrm{Cu}^{2+}$ doped silica shell fusion $\left(\mathrm{Cu}^{2+}\right.$ added time was 10 min later than that of TEOS).

speed, the counter ion interaction strength, the nucleation speed, and the silica shell layers, shell thickness as shown in figure 3.8. All this is critical to future 
application in drug delivery, catalyst loading, and sensors, and so on.

\subsubsection{Synthesis of silica in pure water}

With our above understanding and control, it is easily to realize that base is just a catalyst to help polysilicic acid nucleation because of counter ion interaction, and the solubility decreasing. So we can still control the silica shell thickness after etching by tuning the silica synthesis condition: TEOS incubation time in pure water before adding ammonia to catalyze the TEOS fast hydrolysis and precipitation toward to the $1^{\text {st }}$ stage nucleation. This TEOS incubation in pure DI water control can help to control the ion doping extent inside the silica inner layer, and which will give different shell thickness and porosity there.

According to figure 3.9 it is clear to see the trend change during the stober silica formation in aqueous solution: when the solvent ratio of Water/IPA $=0.2$ (figure 3.9d) the size of silica nanoparticle is largest nearly $250 \mathrm{~nm}$, then the etching in DI water did not give hollow NPs there (figure 3.9h); when the solvent ratio of Water/IPA=0.3 (figure 3.9c) the size of silica nanoparticle is nearly 180 $\mathrm{nm}$, then the etching in DI water gave slightly hollow NPs there (figure $3.9 \mathrm{~g}$ ); when the solvent ratio of Water/IPA=0.4 (figure $3.9 \mathrm{~b}$ ) the size of silica nanoparticle is nearly $140 \mathrm{~nm}$, then the etching in DI water gave heavy hollow NPs there (figure 3.9f); when the solvent ratio of Water/IPA=0.6 (figure 3.9a) the size of silica nanoparticle is nearly $120 \mathrm{~nm}$, then the etching in DI water gave heaviest hollow NPs there (figure 3.9e). 


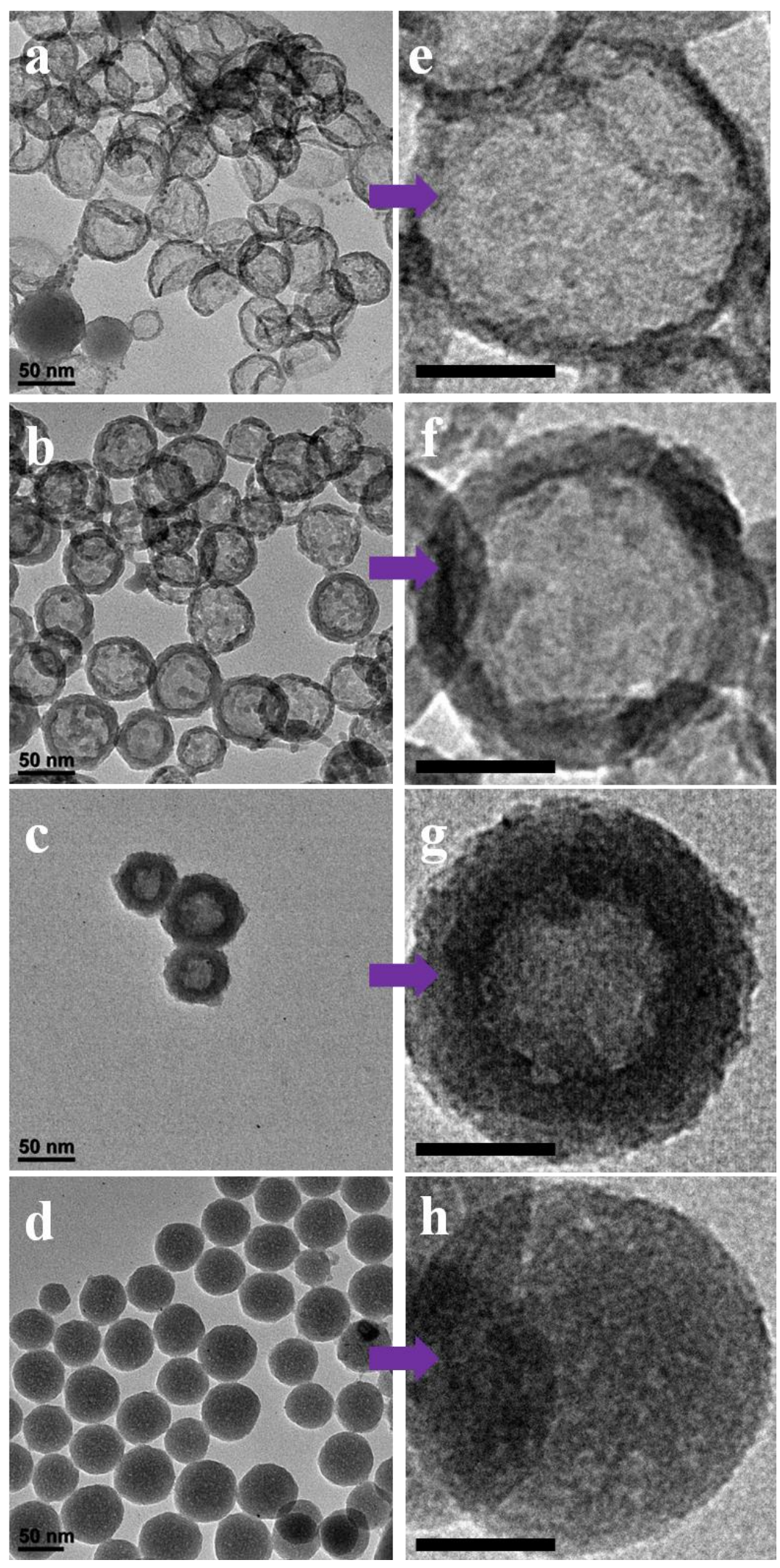

Figure 3.9. TEM images of silica after etching, the synthesized silica was synthesized by TEOS incubated (a) $0 \mathrm{~min}$; (b) $30 \mathrm{~min}$; (c) $90 \mathrm{~min}$; and (d) 240 
min in pure DI water. Scale bar100 nm.

The shell thickness (Figure 3.9) is increasing with the incubation which means that the silica crosslinking and porosity can be tuned by TEOS incubation and ion doping control.

\subsection{Conclusion}

In summary, we explore the generality of the substrate bound Au nanowires growth in terms of the solvent, substrate and ligand. The solvent mainly affect the growth by affecting the reduction rate, and a variety of organic solvent can be used to replace the ethanol with shift of the window of the combination in this system. Various oxide substrates were demonstrated to be capable for the $\mathrm{Au}$ nanowire growth. This generality enables the potential application including preparation of electrode or conductive coating. The generality of ligands were also studied. Aromatic ligands which do not containing any aurophilic substituents are capable to induce the Active Surface growth. Aliphatic ligands, or ligands whose backbone aromatic groups are connected by an $\sigma$ bond would have a problem of packing which compromise their ability to inhibit $\mathrm{Au}$ deposition, and thus failed to generate a constant Active surface for nanowire growth. Substituent group on the benzene ring should have minor effect. 


\subsection{Bibliography}

(1) Purkayastha, A.; Baruah, J. B. Appl. Organomet. Chem. 2004, 18, 166.

(2) Despas, C.; Walcarius, A.; Bessière, J. Talanta 1997, 45, 357.

(3) Green, M.; Harries, J.; Wakefield, G.; Taylor, R. J. Am. Chem. Soc. 2005, 127, 12812.

(4) Kobayashi, Y.; Misawa, K.; Kobayashi, M.; Takeda, M.; Konno, M.; Satake, M.; Kawazoe, Y.; Ohuchi, N.; Kasuya, A. Colloids Surf., A 2004, 242, 47.

(5) Sadasivan, S.; Fowler, C. E.; Khushalani, D.; Mann, S. Angew. Chem. Int. Ed. 2002, 41, 2151.

(6) Alexander, G. B.; Heston, W. M.; Iler, R. K. J. Phys. Chem. 1954, 58, 453.

(7) Slowing, I. I.; Trewyn, B. G.; Giri, S.; Lin, V. S. Y. Adv. Funct. Mater. 2007, $17,1225$.

(8) Bae, S. W.; Tan, W.; Hong, J.-I. Chem. Commun. 2012, 48, 2270.

(9) Sartbaeva, A.; Haines, J.; Cambon, O.; Santoro, M.; Gorelli, F.; Levelut, C.; Garbarino, G.; Wells, S. A. Phys. Rev. B 2012, 85, 064109.

(10) Ma, K.; Sai, H.; Wiesner, U. J. Am. Chem. Soc. 2012, 134, 13180.

(11) Slowing, I. I.; Vivero-Escoto, J. L.; Trewyn, B. G.; Lin, V. S. Y. J. Mater. Chem. 2010, 20, 7924.

(12) Kresge, C. T.; Leonowicz, M. E.; Roth, W. J.; Vartuli, J. C.; Beck, J. S. Nature 1992, 359, 710.

(13) Sartbaeva, A.; Wells, S. A.; Treacy, M. M. J.; Thorpe, M. F. Nat Mater 2006, 
$5,962$.

(14) Fang, X.; Zhao, X.; Fang, W.; Chen, C.; Zheng, N. Nanoscale 2013, 5, 2205.

(15) Zhang, Q.; Zhang, T.; Ge, J.; Yin, Y. Nano Lett. 2008, 8, 2867.

(16) Lou, X. W.; Wang, Y.; Yuan, C.; Lee, J. Y.; Archer, L. A. Adv. Mater. 2006, $18,2325$.

(17) Zhang, Q.; Wang, W.; Goebl, J.; Yin, Y. Nano Today 2009, 4, 494.

(18) Dove, P. M.; Han, N.; Wallace, A. F.; De Yoreo, J. J. Proc. Natl. Acad. Sci. 2008, 105, 9903.

(19) Lou, X. W.; Archer, L. A.; Yang, Z. Adv. Mater. 2008, 20, 3987.

(20) Cheng, K.; Sun, S. Nano Today 2010, 5, 183.

(21) Yec, C. C.; Zeng, H. C. J. Mater. Chem. A 2014, 2, 4843.

(22) Roca, M.; Haes, A. J. J. Am. Chem. Soc. 2008, 130, 14273.

(23) Du, J.; Qi, J.; Wang, D.; Tang, Z. Energy Environ. Sci. 2012, 5, 6914.

(24) Dong, Z.; Lai, X.; Halpert, J. E.; Yang, N.; Yi, L.; Zhai, J.; Wang, D.; Tang, Z.; Jiang, L. Adv. Mater. 2012, 24, 1046.

(25) Wong, Y. J.; Zhu, L.; Teo, W. S.; Tan, Y. W.; Yang, Y.; Wang, C.; Chen, H. J. Am. Chem. Soc. 2011, 133, 11422.

(26) Li, H.; Xin, H. L.; Muller, D. A.; Estroff, L. A. Science 2009, 326, 1244.

(27) Estroff, L. A.; Cohen, I. Nat Mater 2011, 10, 810.

(28) Bogush, G. H.; Tracy, M. A.; Zukoski Iv, C. F. J. Non-Cryst. Solids 1988, $104,95$. 
(29) Crerar, D. A.; Axtmann, E. V.; Axtmann, R. C. Geochim. Cosmochim. Acta 1981, $45,1259$.

(30) Bishop Jr, A. D.; Bear, J. L. Thermochim. Acta 1972, 3, 399.

(31) Pontoni, D.; Narayanan, T.; Rennie, A. R. Langmuir 2002, 18, 56.

(32) Nozawa, K.; Gailhanou, H.; Raison, L.; Panizza, P.; Ushiki, H.; Sellier, E.; Delville, J. P.; Delville, M. H. Langmuir 2004, 21, 1516.

(33) Iler, R. K. J. Colloid Interface Sci. 1980, 75, 138.

(34) Voorhees, P. W. J. Stat. Phys. 1985, 38, 231.

(35) Harris, M. T.; Brunson, R. R.; Byers, C. H. J. Non-Cryst. Solids 1990, 121, 397.

(36) Zhang, T.; Ge, J.; Hu, Y.; Zhang, Q.; Aloni, S.; Yin, Y. Angew. Chem. Int. Ed. 2008, 47, 5806.

(37) Rimstidt, J. D. Geochim. Cosmochim. Acta 1997, 61, 2553.

(38) Stöber, W.; Fink, A.; Bohn, E. J. Colloid Interface Sci. 1968, 26, 62.

(39) Wong, Y. J.; Zhu, L.; Teo, W. S.; Tan, Y. W.; Yang, Y.; Wang, C.; Chen, H. J. Am. Chem. Soc. 2011, 133, 11422.

(40) Chan, S. H. Geothermics 1989, 18, 49.

(41) Dove, P. M.; Crerar, D. A. Geochim. Cosmochim. Acta 1990, 54, 955.

(42) Dove, P. M.; Han, N.; De Yoreo, J. J. Proc. Natl. Acad. Sci. 2005, 102, 15357.

(43) Sjöberg, S. J. Non-Cryst. Solids 1996, 196, 51. 
(44) Iler, R. K. J. Colloid Interface Sci. 1973, 43, 399.

(45) Li, L.; Zhang, L.; Xing, S.; Wang, T.; Luo, S.; Zhang, X.; Liu, C.; Su, Z.;

Wang, C. Small 2013, 9, 825.

(46) Fleming, B. A. J. Colloid Interface Sci. 1986, 110, 40.

(47) Bloomfield, V. A. Biopolymers 1991, 31, 1471.

(48) Manning, G. S. Biophys. Chem. 2002, 101-102, 461.

(49) Cooper, C. L.; Dubin, P. L.; Kayitmazer, A. B.; Turksen, S. Curr. Opin. Colloid Interface Sci. 2005, 10, 52.

(50) Arscott, P. G.; Li, A.-Z.; Bloomfield, V. A. Biopolymers 1990, 30, 619.

(51) Lis, J. T. In Methods Enzymol.; Lawrence Grossman, K. M., Ed.; Academic Press: 1980; Vol. Volume 65, p 347.

(52) Lis, J. T.; Schleif, R. Nucleic Acids Res. 1975, 2, 383.

(53) Homenick, C. M.; Sivasubramaniam, U.; Adronov, A. Polym. Int. 2008, 57, 1007.

(54) Frenkel, D.; Smit, B. Mol. Phys. 1992, 75, 983.

(55) Zhang, Y.; Cremer, P. S. Curr. Opin. Chem. Biol. 2006, 10, 658.

(56) Zhang, Y.; Furyk, S.; Bergbreiter, D. E.; Cremer, P. S. J. Am. Chem. Soc. 2005, 127, 14505.

(57) de la Cruz, M. O.; Belloni, L.; Delsanti, M.; Dalbiez, J. P.; Spalla, O.; Drifford, M. J. Chem. Phys. 1995, 103, 5781.

(58) Garvey, M. J.; Robb, I. D. J. Chem. Soc. Faraday Trans. 1979, 75, 993. 
(59) Richards, R. B. Trans. Faraday Society 1946, 42, 10.

(60) Eliassaf, J. J. Appl. Polym. Sci. 1963, 7, S9.

(61) Shang, L.; Bian, T.; Zhang, B.; Zhang, D.; Wu, L.-Z.; Tung, C.-H.; Yin, Y.; Zhang, T. Angew. Chem. Int. Ed. 2014, 53, 250.

(62) Li, J.; Liang, X.; Joo, J. B.; Lee, I.; Yin, Y.; Zaera, F. J. Phys. Chem. C 2013, $117,20043$.

(63) Fang, X.; Liu, Z.; Hsieh, M.-F.; Chen, M.; Liu, P.; Chen, C.; Zheng, N. ACS Nano 2012, 6, 4434.

(64) Lee, J.-S.; Joo, S. H.; Ryoo, R. J. Am. Chem. Soc. 2002, 124, 1156.

(65) Arriagada, F. J.; Osseo-Asare, K. J. Colloid Interface Sci. 1995, 170, 8.

(66) Song X. H., Ding T., Chen H. Y., Small 2015, 11, 4351-4365. 


\section{Chapter 4 Exploring Ion Pairing Theory into Metal-Silica Janus synthesis}

\subsection{Introduction}

Named after the two-faced Roman God, Janus nanoparticle exhibits two faces of distinct chemical properties. It is also a fundamental structural feature where two materials are intimately joined. With dissymmetric compositions or functionalities, Janus nanoparticles have been studied for synergistic effects and site-specific growth/assembly. ${ }^{1}$ They have been used as surfactants, ${ }^{2}$ sensors, ${ }^{3}$ drug delivering vehicles, ${ }^{4}$ and basic blocks for building superstructures. ${ }^{5,6}$ Despite intensive research, there are still great challenges in the synthesis of Janus nanoparticles. Typical methods involved either partial functionalization ${ }^{7}$ or partial encapsulation of nanoparticles. ${ }^{8}$ But the methods were often developed for a specific type of particles, limiting the scope and versatility.

For hydrophilic nanoparticles, several methods are available to break the symmetry in growing an additional domain on their surface. For example, half encapsulation of silica/polymer can be achieved on nanoparticles with competitive ligand coverage $;^{9-11}$ after trapping nanoparticles at an interface, specific growth or functionalization can occur at only one side; ${ }^{12}$ when depositing metal on the surface of oxide nanoparticles, the large lattice mismatch causes the metal to form an island domain; ${ }^{13-15}$ and facet-specific 
galvanic replacement at the tip of nanorods can give matchstick-like Janus nanocrystals. $^{16-18}$

However, for hydrophobic nanoparticles such as quantum dots or magnetic nanoparticles, there were few successes in breaking their symmetry and achieving Janus structures. ${ }^{12,19}$ Indeed, few methods are available even for the full encapsulation of such nanoparticles. The most notable method is the reverse emulsion method. ${ }^{20,21}$ To date, all such methods in the literature always lead to full encapsulation and thus core-shell structures.

In our recent review, ${ }^{22}$ we argue that the partial or full encapsulation of nanoparticles is often determined by thermodynamics. Just like amorphous nanomaterials often ended up as spherical nanoparticles with the minimal S/V ratio, the growth of a new domain on nanoparticle surface can follow a minimal energy pathway, leading to similar phenomena as the wetting of liquid domains. Basically, a simple encapsulation process involves three interfaces: the seed-shell, seed-solvent, and shell-solvent interfaces. To minimize the overall interfacial energy $\left(\sum \gamma_{i j} \mathrm{~A}_{i j}\right)$, the competition among the three interfaces determines whether full or partial encapsulation occurs. ${ }^{23}$ The seed-shell interfacial energy is often the key factor. It can be modulated by surface ligands, ${ }^{24,25}$ lattice mismatch, ${ }^{26-29}$ interfacial defects, ${ }^{30,31}$ etc.

So based on our understanding on the silica nanoparticles formation mechanism we try to broad and design the new methodology for silica coating to 
form core-shell nanostructures in different environments, such as Stöber system, reverse emulsion system, as well as other systems. Besides, we hope to test the ion-pairing behavior in the other oxide core-shell nanostructures, such as $\mathrm{ZnO}$, $\mathrm{TiO}_{2}$, as well as $\mathrm{CuO}$. We hope to compare the normal different oxides and check whether the similar ion pairing exists in all oxides nanoparticles.

Here, we show that partial encapsulation of hydrophobic nanoparticles in reverse emulsion can be achieved by controlling the solvent ratio, giving concentric, eccentric, or Janus nanoparticles. This new method and understanding open doors to Janus design, and synthesis, broadening the horizon of our search for application. With the surface wetting control (interfacial energy) different level of silica expansion happens on the metal nanoparticle surface.

\subsection{Materials and methods}

\subsubsection{Materials}

All chemical reagents were purchased and used without further purification.

Sodium oleate $\geq 99 \%$ (GC), FeCl3, Oleic acid $\geq 99 \%$ (GC), 1-octadecene $\geq 95.0 \%$ (GC), and tetraethyl orthosilicate (TEOS) were purchased from Sigma Aldrich; Ammonia 25\%-28\% w/w was purchased from Chemical Reagent; Oleic acid-stabilized iron oxide $\mathrm{NP}\left(\mathrm{Fe}_{3} \mathrm{O}_{4} \mathrm{NPs}, \mathrm{d}=40 \mathrm{~nm}\right)$ was purchased from Ocean NanoTech LLC; Hydrogen tetrachloroaurate(III) hydrate $\left(\mathrm{HAuCl}_{4} \cdot 3 \mathrm{H}_{2} \mathrm{O}\right), 99.9 \%$ (metal basis $\mathrm{Au} 49 \%$ ) was purchased from Alfa Aesar; sodium citrate tribasic dihydrate $(99.0 \%)$ were purchased from Sigma-Aldrich; cyclohexane was 
purchased from; CdSe QDs, and CdS QDs were purchased from ; 2-isopropoyol was purchased from ; Igepal-CO520 was purchased from Sigma. Copper specimen grids (200 meshes) with formvar/carbon support film were purchased Beijing Xin Xing Bai Rui Technology Co. Transmission electron microscopy (TEM) images were collected on a JEM-1400 (JEOL) operated at $100 \mathrm{kV}$; Deionized water (resistance $>18.2 \mathrm{M} \Omega \bullet \mathrm{cm}^{-1}$ ) was used in all reactions.

Characterization. Transmission electron microscopy (TEM) images were collected on a JEM-1400 (JEOL) operated at $100 \sim 120 \mathrm{kV}$. Field emission scanning electron microscopy (SEM) images were collected on a JEOL JSM-6700F.

\subsubsection{Methods}

Preparation of TEM Samples. TEM grids were treated with oxygen plasma in a Harrick plasma cleaner/sterilizer for $45 \mathrm{~s}$ to improve the surface hydrophilicity. The hydrophilic face of the TEM grid was then placed in contact with the sample solution. A filter paper was used to wick off the excess solution on the TEM grid, which was then dried in air for $30 \mathrm{~min}$.

Synthesis of $40 \mathrm{~nm}$ Au nanoparticles. Citrate-stabilized Au AuNPs with diameter in $15 \mathrm{~nm}$ and $40 \mathrm{~nm}$ were synthesized according to established literature procedure ${ }^{32}$ with minor modification. For a typical synthesis of $15 \mathrm{~nm}$ AuNPs, $100 \mathrm{ml} \mathrm{HAuCl}$ solution $(0.1 \mathrm{mg} / \mathrm{mL})$ was added to a $250 \mathrm{~mL}$ round bottom flask. This solution was then heated to boil under stirring. $2.4 \mathrm{~mL}$ of 
sodium citrate tribasic dihydrate $(1 \mathrm{wt} \%)$ was added to the solution and heated for another $30 \mathrm{~min}$. The dark-red solution that results suggests the formation of AuNPs. For the synthesis of $40 \mathrm{~nm}$ AuNPs, the procedure is the same as for 15 $\mathrm{nm}$ AuNPs except that $1.5 \mathrm{~mL}$ of sodium citrate tribasic dehydrate solution was added.

Synthesis of the Au@silica concentric nanoparticles. As-synthesized 40 $\mathrm{nm}$ citrate-stabilized AuNP solution $(3 \mathrm{~mL})$ was concentrated to $10 \mu \mathrm{L}$ by centrifugation for $10 \mathrm{~min}$ at 8,000 rpm. The supernatant was then removed and the residual NPs re-dispersed into $50 \mu \mathrm{L}$ of water. The resulting solution was transferred to an $8 \mathrm{~mL}$ viral containing the mixture of $1.5 \mathrm{~mL}$ cyclohexane, and $150 \mu \mathrm{L}$ Igepal CO-520 surfactant (the mixture was mixed by vortex 30 seconds before $\mathrm{Au}$ aqueous solution transfer). Then the viral solution is under vortex for another 30 seconds to give a clear light transparent solution, which means that the reverse emulsion is stable and uniform. Then $15 \mu \mathrm{L}$ ammonia $(25 \%-28 \%$ w/w) was added into the viral followed by another 30 seconds vortex to give light transparent solution. Finally, 6-8 $\mu \mathrm{L}$ pure TEOS was added into the viral and then vortex 30 seconds to make it disperse uniform in the solution. The incubation time is 10-12 hours. After the incubation, the reaction solution was concentrated to $10-15 \mu \mathrm{L}$ by centrifugation for $10 \mathrm{~min}$ at $8,000 \mathrm{rpm}$, then washing two times using ethanol to remove the organic solvent, and surfactant (Igepal CO-520). Then the final precipitation was washed by DI water two 
times to remove the core-free silica nanoparticles, as well as other residues. The intermediates purification process is similar.

Synthesis of the Au@silica eccentric nanoparticles. As-synthesized 40 nm citrate-stabilized AuNP solution $(3 \mathrm{~mL}$ ) was concentrated to $10 \mu \mathrm{L}$ by centrifugation for $10 \mathrm{~min}$ at 8,000 rpm. The supernatant was then removed and the residual NPs re-dispersed into a mixed solvent of $50 \mu \mathrm{L}$ water and $150 \mu \mathrm{L}$ 2-isopropoyol. The resulting solution was transferred to an $8 \mathrm{~mL}$ viral containing the mixture of $1.5 \mathrm{~mL}$ cyclohexane, and $150 \mu \mathrm{L}$ Igepal CO-520 surfactant (the mixture was mixed by vortex 30 seconds before Au aqueous solution transfer). Then the viral solution is under vortex for another 30 seconds to give a clear light transparent solution, which means that the reverse emulsion is stable and uniform. Then $15 \mu \mathrm{L}$ ammonia (25\%-28\% w/w) was added into the viral followed by another 30 seconds vortex to give light transparent solution. Finally, 6-8 $\mu \mathrm{L}$ pure TEOS was added into the viral and then vortex 30 seconds to make it disperse uniform in the solution. The incubation time is 10-12 hours. After the incubation, the reaction solution was concentrated to $10-15 \mu \mathrm{L}$ by centrifugation for $10 \mathrm{~min}$ at $4000 \mathrm{~g}$, then washing two times using ethanol to remove the organic solvent, and surfactant (Igepal CO-520). Then the final precipitation was washed by DI water two times to remove the core-free silica nanoparticles, as well as other residues. The intermediates purification process is similar. 
Synthesis of the Au@silica Janus nanoparticles. As-synthesized $40 \mathrm{~nm}$ citrate-stabilized AuNP solution ( $3 \mathrm{~mL}$ ) was concentrated to $10 \mu \mathrm{L}$ by centrifugation for $10 \mathrm{~min}$ at $8,000 \mathrm{rpm}$. The supernatant was then removed and the residual NPs re-dispersed into a mixed solvent of $50 \mu \mathrm{L}$ water and $240 \mu \mathrm{L}$ 2-isopropoyol. The resulting solution was transferred to an $8 \mathrm{~mL}$ viral containing the mixture of $1.5 \mathrm{~mL}$ cyclohexane, and $150 \mu \mathrm{L}$ Igepal CO-520surfactant (the mixture was mixed by vortex 30 seconds before $\mathrm{Au}$ aqueous solution transfer). Then the viral solution is under vortex for another 30 seconds to give a clear light transparent solution, which means that the reverse emulsion is stable and uniform. Then $15 \mu \mathrm{L}$ ammonia $(25 \%-28 \% \mathrm{w} / \mathrm{w})$ was added into the viral followed by another 30 seconds vortex to give light transparent solution. Finally, 6-8 $\mu \mathrm{L}$ pure TEOS was added into the viral and then vortex 30 seconds to make it disperse uniform in the solution. The incubation time is 10-12 hours. After the incubation, the reaction solution was concentrated to $10-15 \mu \mathrm{L}$ by centrifugation for $10 \mathrm{~min}$ at $4000 \mathrm{~g}$, then washing two times using ethanol to remove the organic solvent, and surfactant (Igepal CO-520). Then the final precipitation was washed by DI water two times to remove the core-free silica nanoparticles, as well as other residues. The intermediates purification process is similar.

Synthesis of the metal NP@silica concentric nanoparticles. All procedure is similar to Au@silica concentric nanoparticles, except that the hydrophobic 
nanoparticles were dispersed into the cyclohexane, and then mixed with surfactant (Igepal CO-520). Then $50 \mu \mathrm{L}$ water was added into the solution, and 30 seconds vortex the viral to give a stable reverse emulsion. Then $15 \mu \mathrm{L}$ ammonia $(25 \%-28 \% \mathrm{w} / \mathrm{w})$ was added into the viral followed by another 30 seconds vortex to give light transparent solution. Finally, 6-8 $\mu \mathrm{L}$ pure TEOS was added into the viral and then vortex 30 seconds to make it disperse uniform in the solution. The incubation time is $10-12$ hours. The purification process is similar, two times ethanol washing followed by two rounds water washing. For $40 \mathrm{~nm} \mathrm{Fe}_{3} \mathrm{O}_{4} @$ @ilica nanoparticles, centrifugation speed is $8,000 \mathrm{rpm}$; for 20 nm $\mathrm{Fe}_{3} \mathrm{O}_{4} @$ silica nanoparticles, centrifugation speed is $13,000 \mathrm{rpm}$; for other hydrophobic nanoparticles (size between 10-15 nm), then centrifugation speed is $14,000 \mathrm{rpm}$.

Synthesis of the metal NP@silica ecccentric nanoparticles. All procedure is similar to concentric nanoparticles synthesis, except that the solvent ratio is different. Here, we use $50 \mu \mathrm{L}$ water and $150 \mu \mathrm{L}$ 2-isopropoyol mixed solution instead of $50 \mu \mathrm{L}$ DI water.

Synthesis of the metal NP@silica Janus nanoparticles. All procedure is similar to concentric nanoparticles synthesis, except that the solvent ratio is different. Here, we use $50 \mu \mathrm{L}$ water and $240 \mu \mathrm{L}$ 2-isopropoyol mixed solution instead of $50 \mu \mathrm{L}$ DI water. 


\subsubsection{Seed concentration}

In order to control the seed concentration during the silica encapsulation we have tried to use similar seed concentration, similar TEOS concentration, and also keep the surfactant and ammonia quantity the same.

What we did is just two series experiments: one is synthesis of core-shell nanoparticles in reverse emulsion system while tuning the solvent ratio inside the aqueous phase (water/IPA), the compared control experiment was conducted in DI water with the similar solvent ratio. The control experiment help to rule out the interface fabrication of reverse emulsion mechanism; another series experiments was designed to tune the ion concentration inside the aqueous solution when coating metal nanoparticles using reverse emulsion method. The ion concentration (salt concentration) can affect the ion paring during the silica formation, and then lead to shell morphology change.

\subsection{Results and discussion}

Our previous mechanistic studies indicated that the composition of Stöber silica depends on the solvent ratio of the preparative solution. ${ }^{33}$ Hence, we attempt to apply the general principles and study silica formation in a different system, namely the reverse emulsion method $^{21,34,35}$ which is best for encapsulating hydrophobic nanoparticles without causing their aggregation. In this work, we achieve partial silica encapsulation of various nanoparticles by simply adjusting the solvent ratio of the emulsion droplets. The new method is 
general, facile, and scalable, capable of converting 6 types of hydrophobic nanoparticles to Janus structures.

\subsubsection{Iron oxide coating silica in reverse emulsion}

Our previous mechanistic studies indicated that the composition of Stöber silica depends on the solvent ratio of the preparative solution. ${ }^{33}$ Hence, we attempt to apply the general principles and study silica formation in a different system, namely the reverse emulsion method ${ }^{21}, 34,35$ which is best for encapsulating hydrophobic nanoparticles without causing their aggregation. In this work, we achieve partial silica encapsulation of various nanoparticles by simply adjusting the solvent ratio of the emulsion droplets. The new method is general, facile, and scalable, capable of converting 6 types of hydrophobic nanoparticles to Janus structures.

The method of partial encapsulation is adapted from that of full encapsulation. ${ }^{36-38}$ More specifically, oleic acid-stabilized $\mathrm{Fe}_{3} \mathrm{O}_{4}$ nanoparticles were dispersed in cyclohexane, followed by the addition of aqueous $\mathrm{NH}_{3}$ (final $0.12 \mathrm{M})$, 2-isopropanol (IPA), and the surfactant polyoxyethylene nonylphenylether (Igepal-CO-520). The mixture $\left(\mathrm{H}_{2} \mathrm{O} / \mathrm{IPA} / \mathrm{C}_{6} \mathrm{H}_{12}=35: 15: 1000\right)$ was vortexed to generate a stable emulsion. After adding TEOS to initiate silica formation, the emulsion was incubated at room temperature overnight $(8 \mathrm{~h})$. The product nanoparticles were isolated by magnetic attraction (for $\mathrm{Fe}_{3} \mathrm{O}_{4}$ 


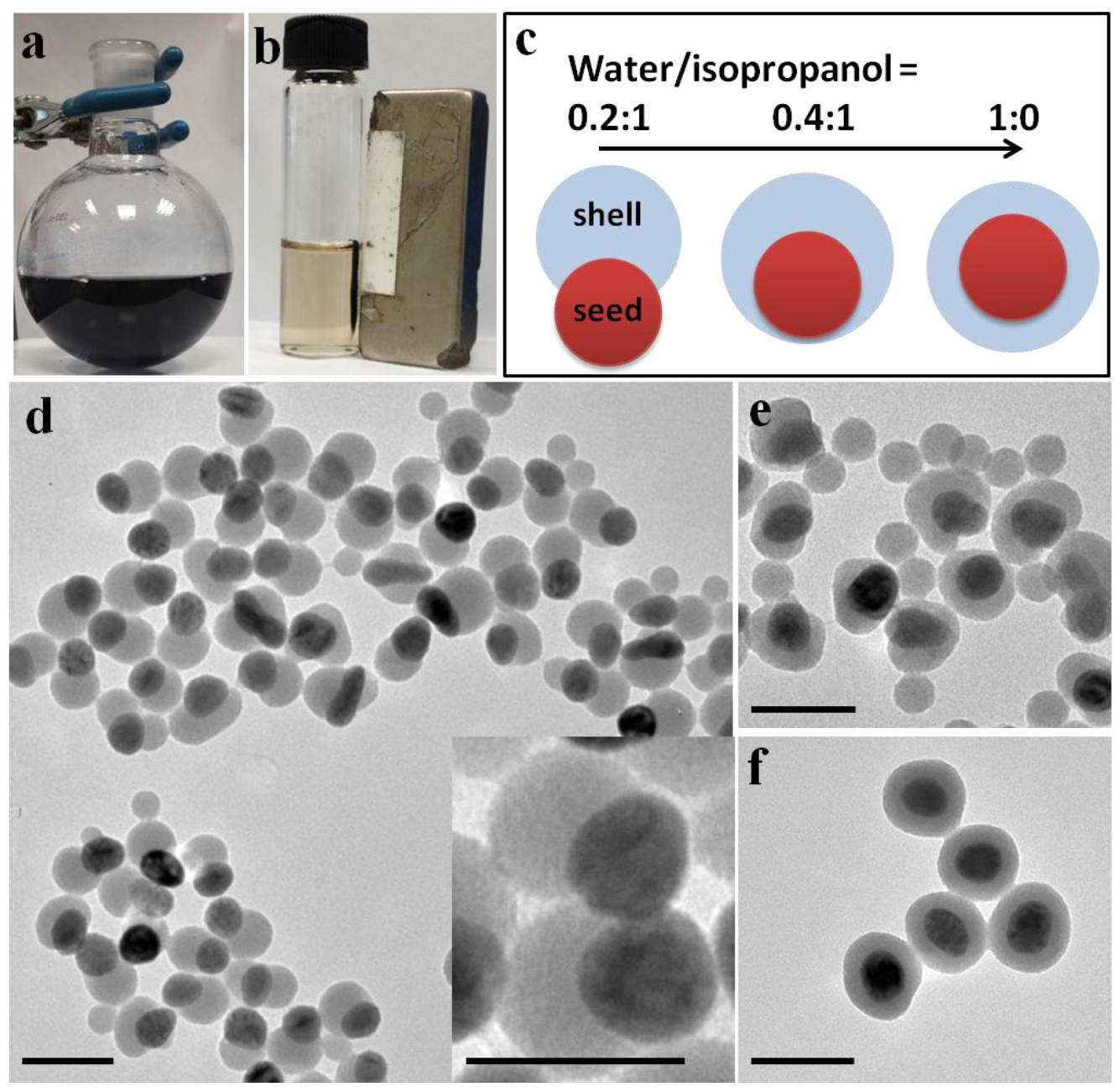

Figure 4.1. The TEM of $40 \mathrm{~nm}$ iron oxide nanoparticles coating under different solvent ratio in reverse emulsion: $\mathrm{H}_{2} \mathrm{O} / \mathrm{IPA} / \mathrm{C}_{6} \mathrm{H}_{12}=$ (d) $10: 40: 1000$, inset is the TEM of Janus NPs under high magnification (e) 35:15:1000, (f) 50:0:1000. Scale bars: $100 \mathrm{~nm}$.

nanoparticles, Figure 1b) or centrifugation, and washed in ethanol. The total volume of $\mathrm{H}_{2} \mathrm{O}$ and IPA was set as $50 \mu \mathrm{L}$ but their ratio was adjusted to achieve different degrees of partial encapsulation. As shown in the TEM images, Janus (Figure 4.1d), eccentric (Figure 4.1e), and concentric core-shell nanoparticles (Figure 4.1f) were prepared under the solvent ratios of 10:40:1000, 35:15:1000, 


\begin{tabular}{|l|c|c|c|c|c|c|c|}
\hline $\begin{array}{c}\text { Initial solvent } \\
\text { ratio } \\
\left(\mathrm{H}_{2} \mathrm{O} / \mathrm{IPA} / \mathrm{C}_{6} \mathrm{H}_{12}\right)\end{array}$ & $\begin{array}{c}\mathrm{V}_{0} \\
\text { initial } \mathrm{H}_{2} \mathrm{O} \\
\text { volume }\end{array}$ & $\begin{array}{c}\mathrm{V}_{\text {IPA }} \\
\text { initial } \\
\text { volume }\end{array}$ & $\begin{array}{c}\mathrm{M}_{3}: \mathrm{M}_{4} \\
\left(\mathrm{~V}_{1}: \mathrm{V}_{2)}\right.\end{array}$ & $\begin{array}{c}\mathrm{V}_{1}=\mathrm{V}_{\text {IPA }} \\
\text { In water }\end{array}$ & $\begin{array}{c}\mathrm{V}_{2}=\mathrm{V}_{\text {IPA }} \\
\text { In oil }\end{array}$ & $\begin{array}{c}\mathrm{V}_{0} \\
\mathrm{H}_{2} \mathrm{O} \text { final } \\
\text { volume }\end{array}$ & $\begin{array}{c}\text { Coating } \\
\text { shape }\end{array}$ \\
\hline $50: 0: 1000$ & $50 \mu \mathrm{L}$ & 0 & 0 & 0 & 0 & $50 \mu \mathrm{L}$ & Concentric \\
\hline $35: 15: 1000$ & $35 \mu \mathrm{L}$ & $15 \mu \mathrm{L}$ & 1.23 & $8.3 \mu \mathrm{L}$ & $6.7 \mu \mathrm{L}$ & $43.3 \mu \mathrm{L}$ & Eccentric \\
\hline $10: 40: 1000$ & $10 \mu \mathrm{L}$ & $40 \mu \mathrm{L}$ & 0.90 & $18.95 \mu \mathrm{L}$ & $21.05 \mu \mathrm{L}$ & $28.95 \mu \mathrm{L}$ & Janus \\
\hline
\end{tabular}

Table 4.1. Solvent ratio and volume change of aqueous phase in reverse emulsion for different solvent ratio system.

and 50:0:1000, respectively. The synthesis can be easily scaled up to fabricate $100 \mathrm{~mL}$ solution of Janus nanoparticles (Figure 4.1a).

IPA can partially dissolve in both the water and cyclohexane phase, making the real solvent ratio different from the nominal ratio of the initial mixture. To understand its partition, the three solvents were mixed in a scale-up experiment without surfactants. The real water/IPA ratio in the aqueous phase was found to be 10:18.9; 35:8.3; and 50:0 for the above three mixtures, respectively (Table 4.1). IPA is only marginally soluble in cyclohexane. Here, a significant amount of IPA stays in the cyclohexane phase because the volume of cyclohexane is much larger than that of water.

Here we can see the nanostructure form concentric to eccentric, and Janus caused by solvent ratio change. It is possible that the Janus was formed at the interface of organic/water phase. So we need to design experiments to find out the real mechanism there. 


\subsubsection{Hydrophilic Au encapsulation in reverse emulsion}

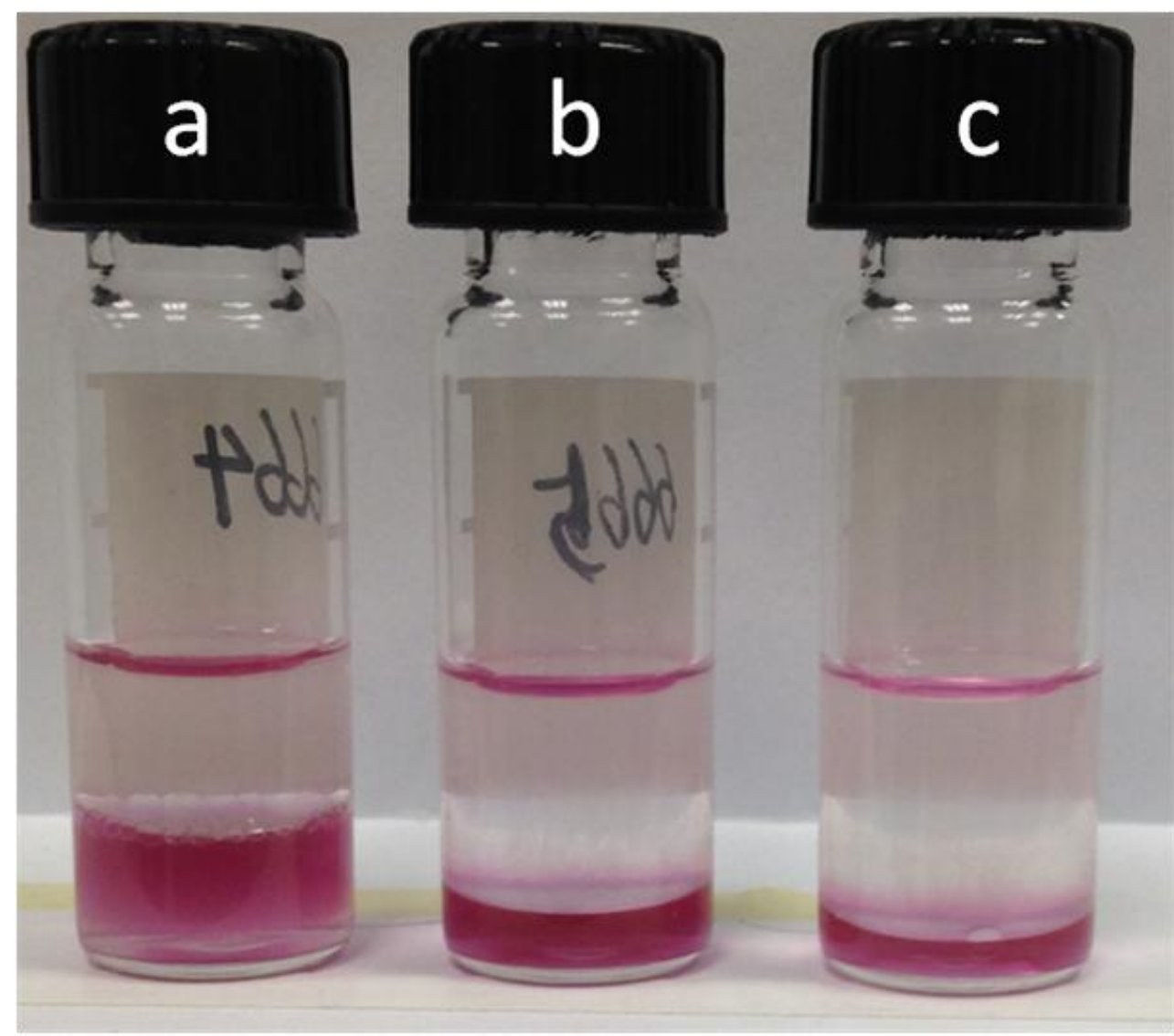

Figure 4.2. Photo of $\mathrm{Au}$ NPs dispersion under different solvent ratio of

IPA/water: (a) 1.0, (b) 0.4, (c) 0.2. The photos show Au disperse in the aqueous phase uniformly but not at the organic/aqueous interface. This can help to rule out the interface coating giving Janus nanoparticles there.

Being a non-polar molecule, TEOS dissolves in the cyclohexane phase and only reacts with water at the emulsion interface, generating the hydrophilic silicic acid in the aqueous phase. The resulting poly(silicic acid) then formed ion pairing with $\mathrm{NH}_{4}{ }^{+}$ions, leading to the nucleation and growth of silica domains. ${ }^{33}$ It is known that the solvent ratio of the Stöber method can affect the degree of ion pairing and thus, the silica composition. However, we cannot 


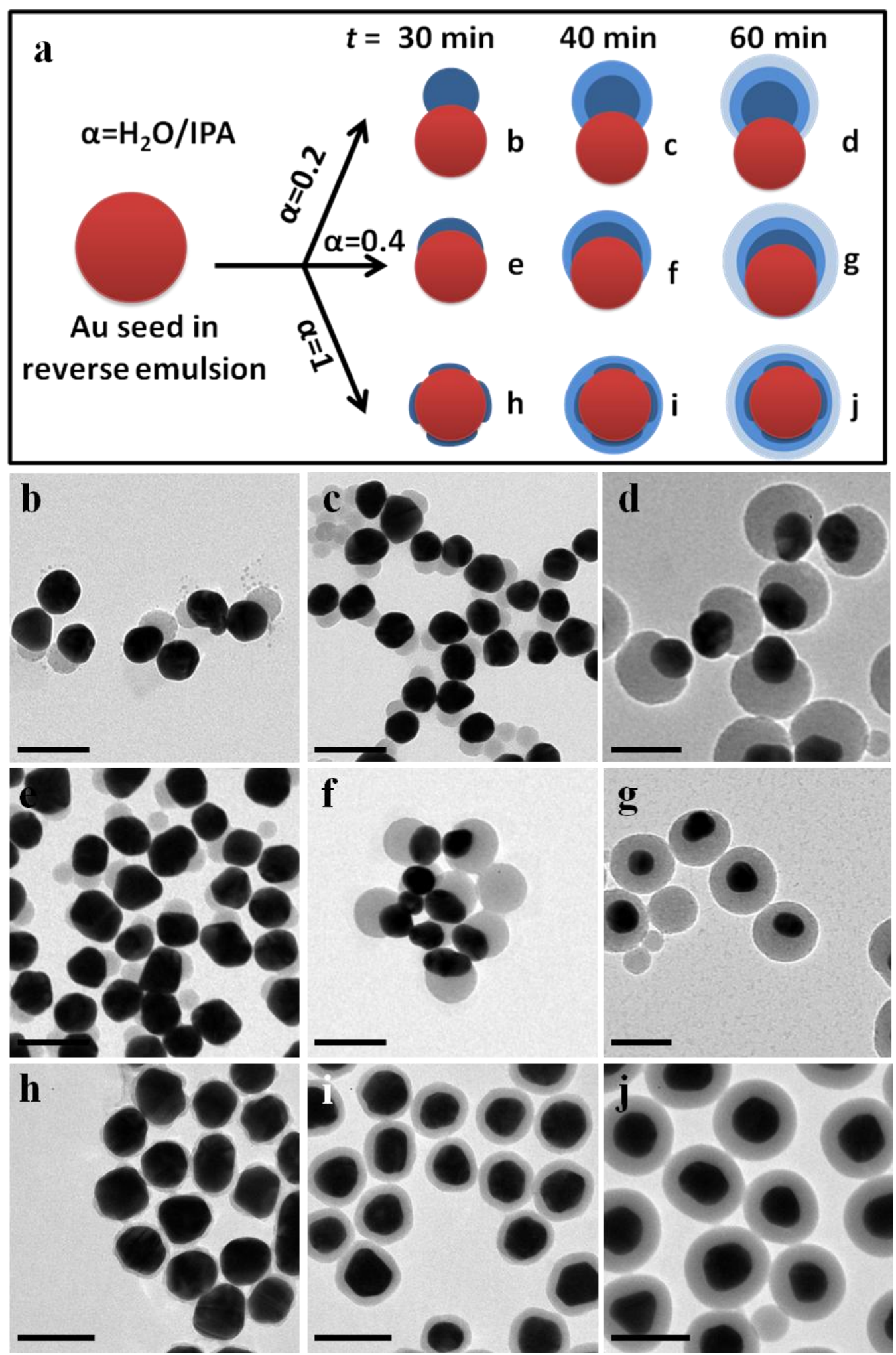

Figure 4.3. The TEM of $40 \mathrm{~nm}$ Au nanoparticles coating under different solvent ratio of $\mathrm{H}_{2} \mathrm{O} / \mathrm{IPA} / \mathrm{C}_{6} \mathrm{H}_{12}$ (a-c) 10:40:1000, (d-f) 35:15:1000, (g-i) 50:0:1000. The intermediates was taken out at different times during the synthesis of: (a) 
$30 \mathrm{~min}$, (b) 40min, (c) 60 min Janus nanoparticles; (d) $30 \mathrm{~min}$, (e) 40min, (f) 60 min eccentric nanoparticles; (g) $30 \mathrm{~min}$, (h) 40min, (i) 60 min concentric nanoparticles. Scale bar: 100nm.

jump to this conclusion here because the extent of ligand loss from the $\mathrm{Fe}_{3} \mathrm{O}_{4}$ nanoparticles is unknown and it may affect the formation of silica domains.

Therefore, we applied the partial encapsulation method to hydrophilic Au nanoparticles, which has clean surface chemistry and is known to stay in the aqueous phase (as opposed to the water/cyclohexane interface). Citrate-stabilized Au nanoparticles of $40 \mathrm{~nm}$ in diameter were treated by 4-mercaptobenzoic acid (MBA) and isolated by centrifugation. ${ }^{39}$ The resulting concentrated solution was mixed with aqueous $\mathrm{NH}_{3}$ and then used as the aqueous phase of the emulsion. The product nanoparticles showed similar Janus, eccentric and concentric structures at the nominal solvent ratio of $\mathrm{H}_{2} \mathrm{O} / \mathrm{IPA} / \mathrm{C}_{6} \mathrm{H}_{12}=10: 40: 1000$, 35:15:1000, and 50:0:1000, respectively.

To obtain a clearer contrast difference between the core and shell, we tried to trap the intermediates of silica encapsulation. Firstly, we coating $\mathrm{Au}$ nanoparticles under different solvent ratio, and then $100 \mu \mathrm{L}$ of the mixture was taken out at different time $\operatorname{span}(\mathrm{t}=30,40$, and $60 \mathrm{~min})$ followed by immediately diluted in $1 \mathrm{~mL}$ DI water and centrifugation followed by washing in pure ethanol (Figure 4.3a) . The immediate dilution and centrifugation can help to quenching the reaction to give intermediates. 
It is clear to see the trends from the TEM images in Figure 4.3b-d, e-g, and h-j. The first trend is the size of silica domain increases with reaction time prolonging. The second trend is the silica-Au contact interface area increases with reaction time (Figure 4.3b-d, and e-g) and water content (Figure 4.3d, g, and j). It is clear that the higher water/IPA/cyclohexane ratio gives high silica expansion speed on the silica-Au contact interface, and that with increasing water content, the contact area of Au-silica in general increases (Figure 4.3d, g, j). We can get the solvent ratio threshold for different Au-silica morphologies here, which can help us to know the relationship between solvent ratio and nanoparticle morphology: when the solvent ratio is $35: 15: 1000<$ water/IPA/cyclohexane <40:10:1000 we get concentric mixed with eccentric core-shell Au@silica nanoparticles; when the solvent ratio is 10:40:1000<water/IPA/cyclohexane<35:15:1000, we get eccentric core-shell Au@silica nanoparticles mixed with Janus Au-silica nanoparticles; when the solvent ratio is water/IPA/cyclohexane $\leq 10: 40: 1000$ we get Janus Au-silica nanoparticles.

\subsubsection{Mechanism study}

The mechanism for silica shell morphology difference in the above system is adjusted here. We think the reason is not because of metal nanoparticle was coated at the interface of oil/aqueous phase giving Janus nanoparticles. The first reason is that we cannot get eccentric core-shell nanoparticles if the coating 


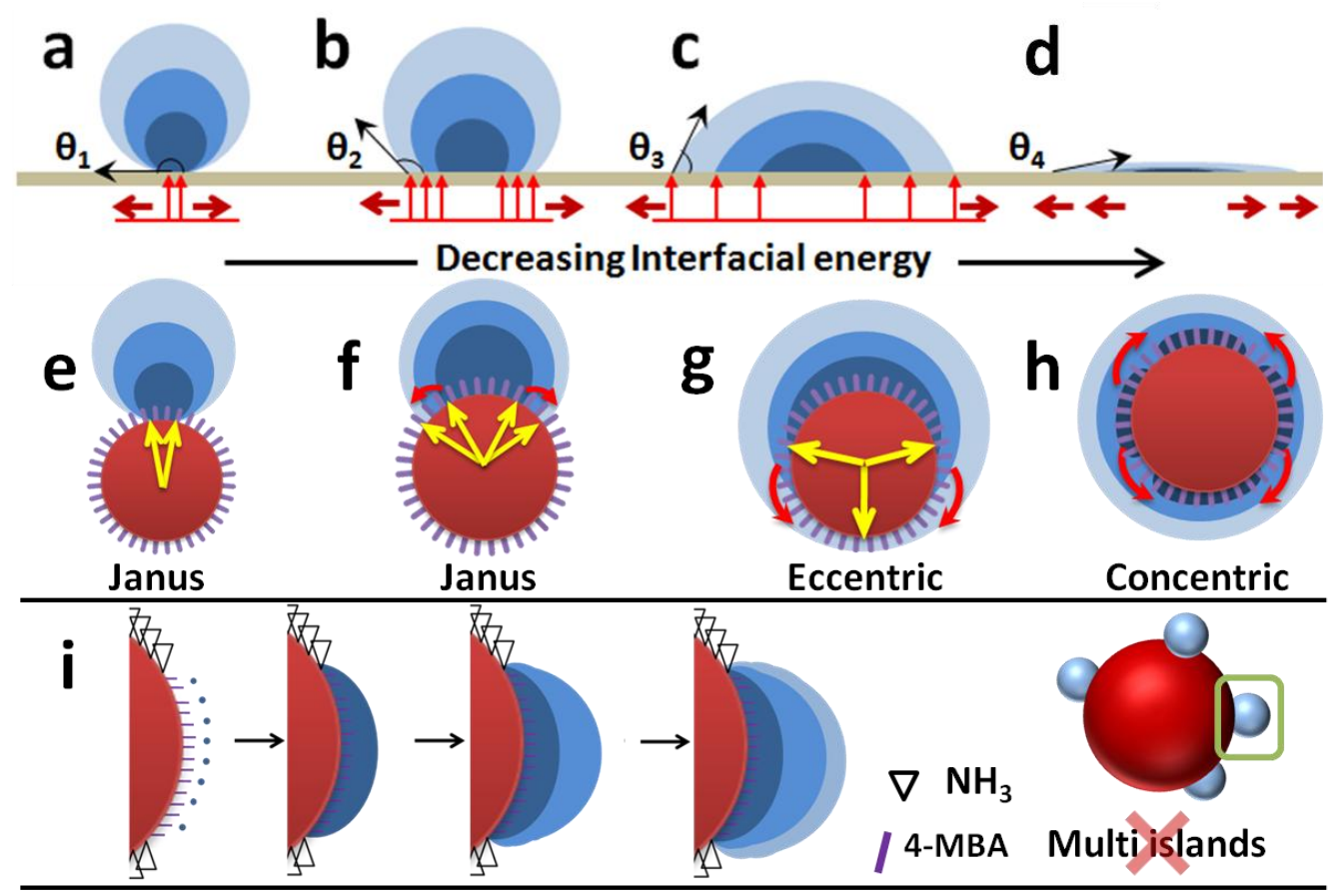

Figure 4.4. The mechanism for silica coating, (a) droplet on the substrate for different surface wetting, (b) silica deposition on the spherical surface under different solvent ratio.

process just happened at the oil/aqueous interface. The second reason is the nanoparticles different surface: Au surface is hydrophilic while the iron oxide surface is hydrophobic. They both can give eccentric and Janus nanoparticles although the surface property is opposite difference. It is impossible that both iron oxide nanoparticles and $\mathrm{Au}$ nanoparticles are locked at the interface for silica coating.

In order to get proof we did a control experiment to show the nanoparticle location in the reverse emulsion during silica coating as shown in figure 4.2. It is clear to see that all Au nanoparticles are dispersed uniformly in aqueous phase 
not at the oil/aqueous interface. So the data can also help to rule out the oil/water interface coating mechanism.

The mechanism is just because silica composition difference caused by solvent ratio tuning during the coating control experiments. The different solvent ratio changes the solvent polarity which is critical to the ion doping during the silica nucleation and deposition. Then the different 'silica' shows different interfacial energy to Au nanoparticle. As show in the figure 4.4, the different 'silica' shells have different ion doping level, which will show different interfacial energy to Au nanoparticles: the high solvent ratio (water/IPA=50:0) gives silica with lower ion doping which shows lower interfacial energy to Au nanoparticles, and concentric core-shell nanoparticles were the final product; the medium solvent ratio (water/IPA=35:15) leads to medium ion doping 'silica' formation which shows medium interfacial energy to Au nanoparticle, and the eccentric core-shell nanoparticle was collected; the lowest solvent ratio (water/IPA=10:40) caused highest ion doping 'silica' formation which shows highest interfacial energy to Au surface, and the Janus nanoparticle is the final product. All this mechanism process is consistent with our experimental data.

Besides the above argument we also consider the effect of the interaction between $\mathrm{Au}$ and silica shell. Is it possible that the surfactant, or original $\mathrm{Au}$ surface ligand dominate the Janus formation? So nanoparticles with different surface ligands was applied in reverse emulsion method, such as iron oxide 
stabilized by Oley amine/oley acid were used; another control is Au stabilized by critate/MBA were used. Both the two control experiments show similar Janus nanoparticle as the final product. Another experiment we study about the interaction effect is surface wetting study, which is viewed in kinetic and thermodynamic theory. we believe that the silica growth is holistic $\square$ that the solid silica domain can somehow "feel" the overall shape when minimizing its interfacial energies. It is actually very similar to the formation of spherical nanoparticles, where silica seems to "know" where to deposit when achieving the shape with the minimal S/V ratio. While the silica domain cannot flow like liquid, it can reach the favored conformation if the surface energy is minimized at each step of growth. Hence, we believe that the partial encapsulation here follows similar minimal energy pathways, approaching the thermodynamically controlled "wetting" conformations. The ligand MBA merely improves the Au-silica interfacial energy, but is not directly responsible for setting the boundary of the partial encapsulation.

A prerequisite for expanding the Au-silica interface is that the silica may "flow" or deposit onto new Au surfaces (Figure 4.5c). We had some doubts on the liquidity of the silica nanomaterials grown in solution, as they differ significantly from pyrogenic silica (glass or sand) in terms of porosity and composition. However, the non-spherical silica domains in Figure 1 are good examples against the liquid proposition. Most importantly, silica prepared under the same 
conditions can be etched hollow. The fact that the remaining shells did not collapse/withdraw into a droplet is strong evidence that the silica even at the swollen state (by the solvent water) behave like a solid.

Careful examination of the Janus nanoparticles revealed that the contact angles of quite a few cases were abnormally large, so that the silica domain appears to "bulge" out (Figure 4.5e-h). This deviation can be likened to the pinning phenomena of water droplet on a substrate. Basically, as water evaporates, the withdrawal of the droplet boundary is non-uniform. When the air-water-substrate contact line is pinned, the evaporation of water can lead to smaller contact angle than the equilibrium angle set by the surface energy (Figure 4.5a).

Similarly, we believe that during the growth of silica domain, the expansion of its boundary is unlikely always uniform. Growth may be sometimes unfavorable at the solvent-Au-silica contact line, possibly due to the inhomogeneity of the local environment (e.g., ligand density). Continual silica growth around the pinned contact line thus forces the silica domain to bulge out (Figure $4.5 \mathrm{~b}$ ). The deviation from the equilibrium contact angle increases the surface energies near the contact line (it would be more favorable to deposit at the concave surface), eventually causing the contact line to advance. As shown in Figure $4.5 \mathrm{~d}$, at the instant of "depinning", more growth should occur near the contact line than the average overcoating on the silica domain, likely promoted by the rapid filling of the concave surface. In other words, the "pinning" and "depinning" when 

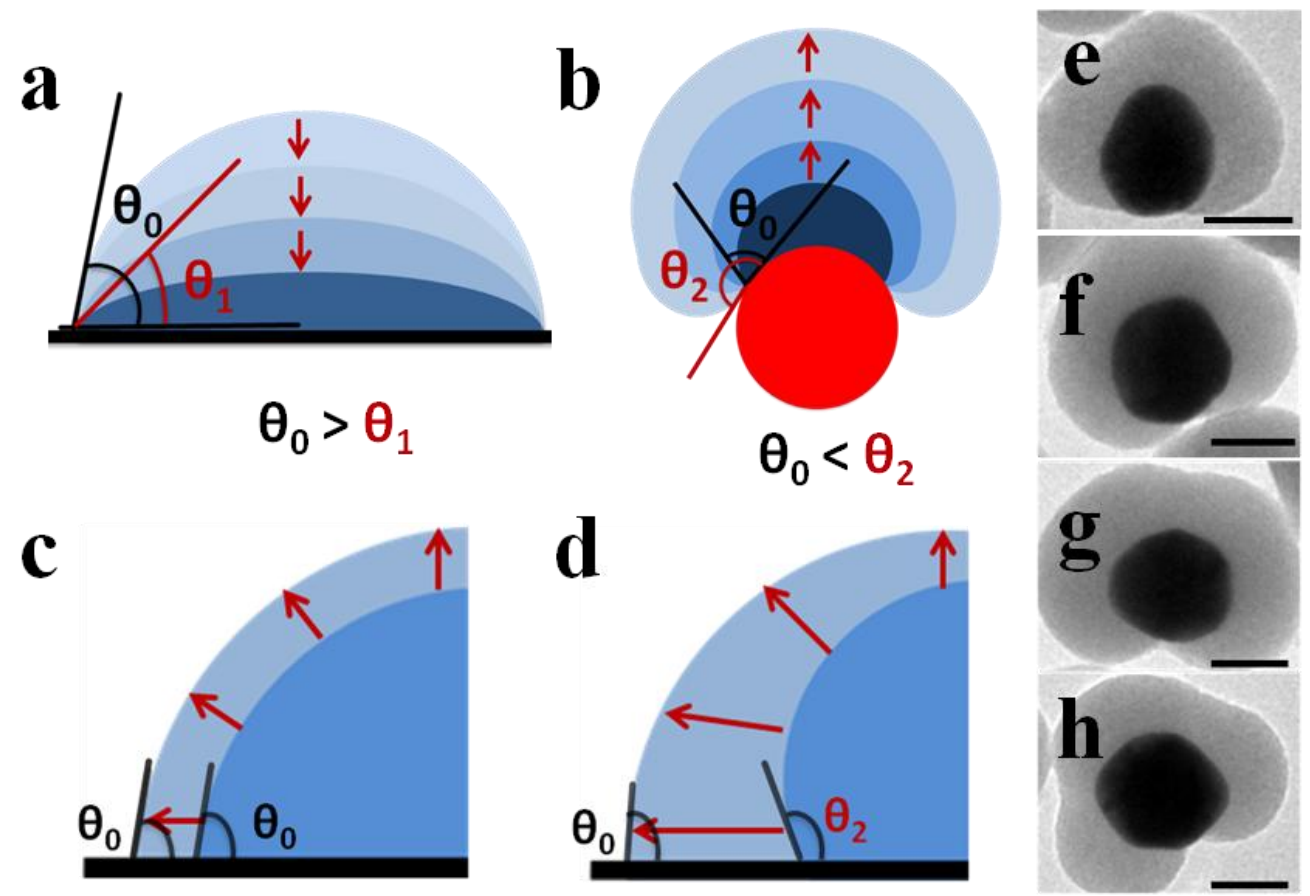

Figure 4.5. Scheme of (a) Constant contact line mode (CCL) during water droplet evaporation: contact angle decreases $(\theta 1<\theta 0)$ with evaporation while contact line is pinned, (b) Constant contact line mode (CCL): silica deposits on curve Au surface and: contact angle increases $(\theta 0<\theta 2)$ with silica deposition, (c) constant contact angle (CCA) growth mode: silica deposit evenly to keep the same equilibrium contact angle, and (d) pining-depinning growth mode: silica deposition faster near the contact line to keep equilibrium contact angle $(\theta 0),(\mathrm{e}-\mathrm{h})$ TEM image of Au-silica while silica bulge out caused by contact line pinning . Scale Bar: $50 \mathrm{~nm}$.

growing the solid domain should have utterly different mechanisms from those during the expansion of liquid domains.

In order to prove the mechanism we design the following experiments: one set experiments are silica synthesis control under the above solvent ratio to check the 
composition difference in Stöber method (silica synthesis and Au@silica synthesis at different solvent ratio in Stöber system); another set experiments are ion doping control by salt concentration control in the reverse emulsion.

\subsubsection{Silica synthesis and etching}

In order to prove silica nanoparticle is different when it was synthesized under different solvent ratio we designed to synthesize silica at Stöber system while tune the solvent ratio of water/IPA, which we think can change the ion doping level during the polysilicic acid nucleation and deposition. Then we did the CHNS elemental analysis to check the $\mathrm{N}$ content at the different samples. Another control is etching the as synthesized silica nanoparticle under different solvent ratios at the same etching condition: the same etching concentration, the same water volume (etching solvent) for etching, the same temperature, and the same time length. We hope to compare the etching phenomenon after etching.

As shown in figure 4.5, the silica nanoparticle synthesized under different solvent ratio have different size and the different $\mathrm{N}$ content: high solvent ratio (water/IPA) leads to lower ion doping which means that less $\mathrm{NH}_{4}{ }^{+}$trapped inside silica, and the lower $\mathrm{N}$ content data is consistent with hypothesis; lower solvent ratio (water/IPA) gives high level ion doping inside silica which means that more $\mathrm{NH}_{4}{ }^{+}$trapped inside silica, and extremely higher $\mathrm{N}$ content data present in the CHNS elemental analysis. 

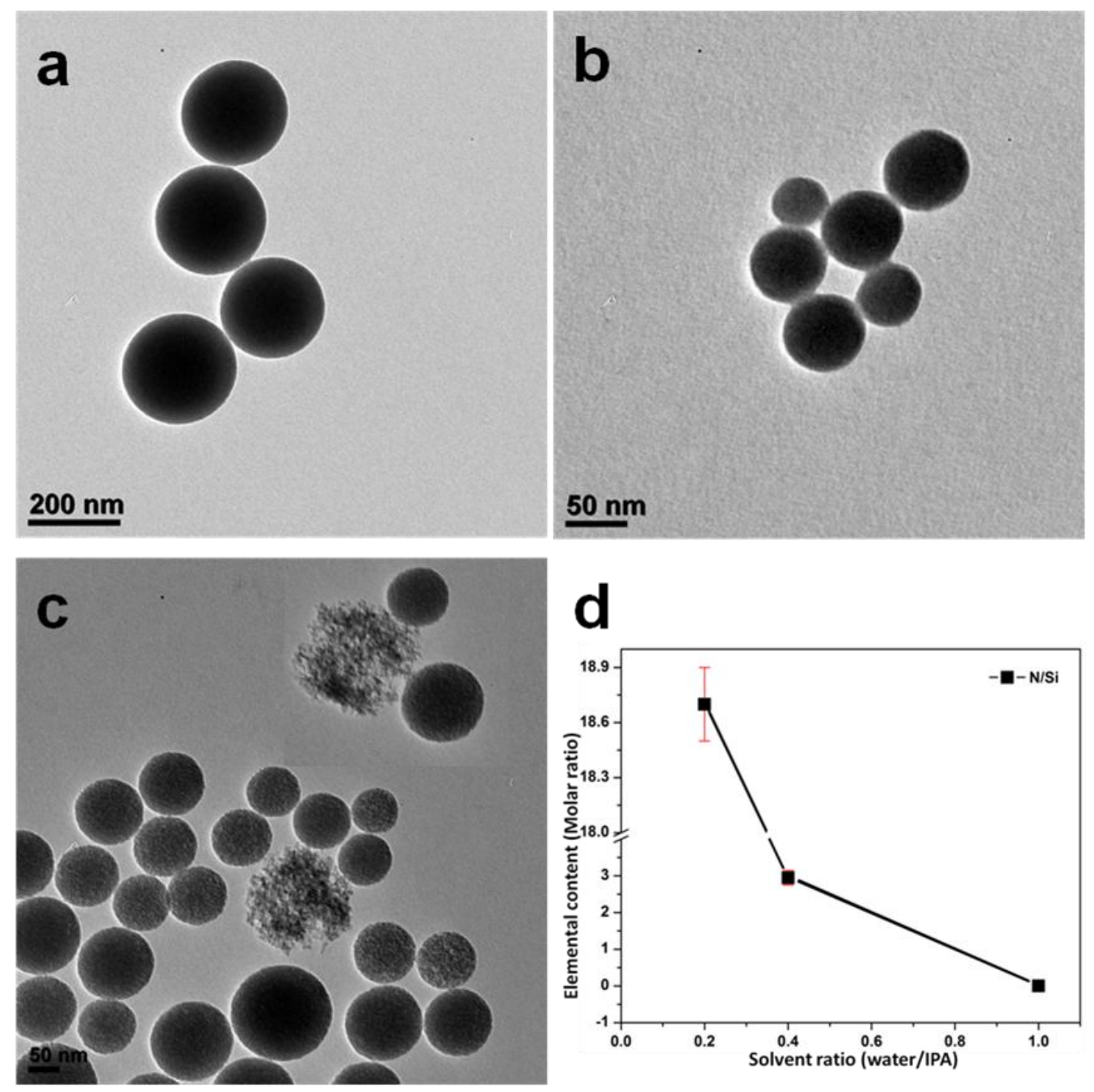

Figure 4.6. TEM image of silica nanoparticles synthesized under different solvent ratio (water/IPA) (a) 0.2, (b) 1.0, and (c) mix sample a and b to etch at $90{ }^{\circ} \mathrm{C}, 30 \mathrm{~min}$. This can show these silica synthesized under different solvent ratio have different etching behaviour which means that this are different 'silica'.

Besides, the etching of as-synthesized silica nanoparticles at different solvent ratio can also prove the hypothesis: different solvent ratio gives different kinds of silica nanoparticles. As shown in figure 4.6, we mixed the silica nanoparticles synthesized at different solvent ratio (water/IPA=0.2, 1.0) together, and then do etching in one vial under the standard etching condition. The etching behavior is 
obviously different for the two different 'silica': The silica synthesized under higher solvent ratio (smaller silica) is hollow while the etching speed is slow; the silica synthesized under lower solvent ratio (larger silica) is uniformly etched while the etching speed is faster. The reason is that 'silica' synthesized under higher solvent ratio is lower ion doping and difficult to etch, but the silica synthesized under lower solvent ratio is heavier ion doping and easy to etch. All the experimental phenomena are consistent with our hypothesis of different kinds of 'silica' under different solvent ratio. The 'silica' elemental analysis and etching data can prove our hypothesis.

\subsubsection{Au NP coating by stöber method}

Here we try to prove the hypothesis directly in Stöber system. We tried to coating Au nanoparticles under different solvent ratios (water/IPA) by Stöber method.

$15 \mathrm{~nm} \mathrm{Au}$ nanoparticles and $40 \mathrm{~nm}$ Au nanoparticles were synthesized and used as seed to coating silica shell by Stöber method. By using different size of Au nanoparticles here we can rule out the reason of nanoparticle size effects on the silica shell deposition and formation. The Au nanoparticles we used here were treated similarly as the Au nanoparticles we used in the reverse emulsion system in order to compare the shell morphology. 


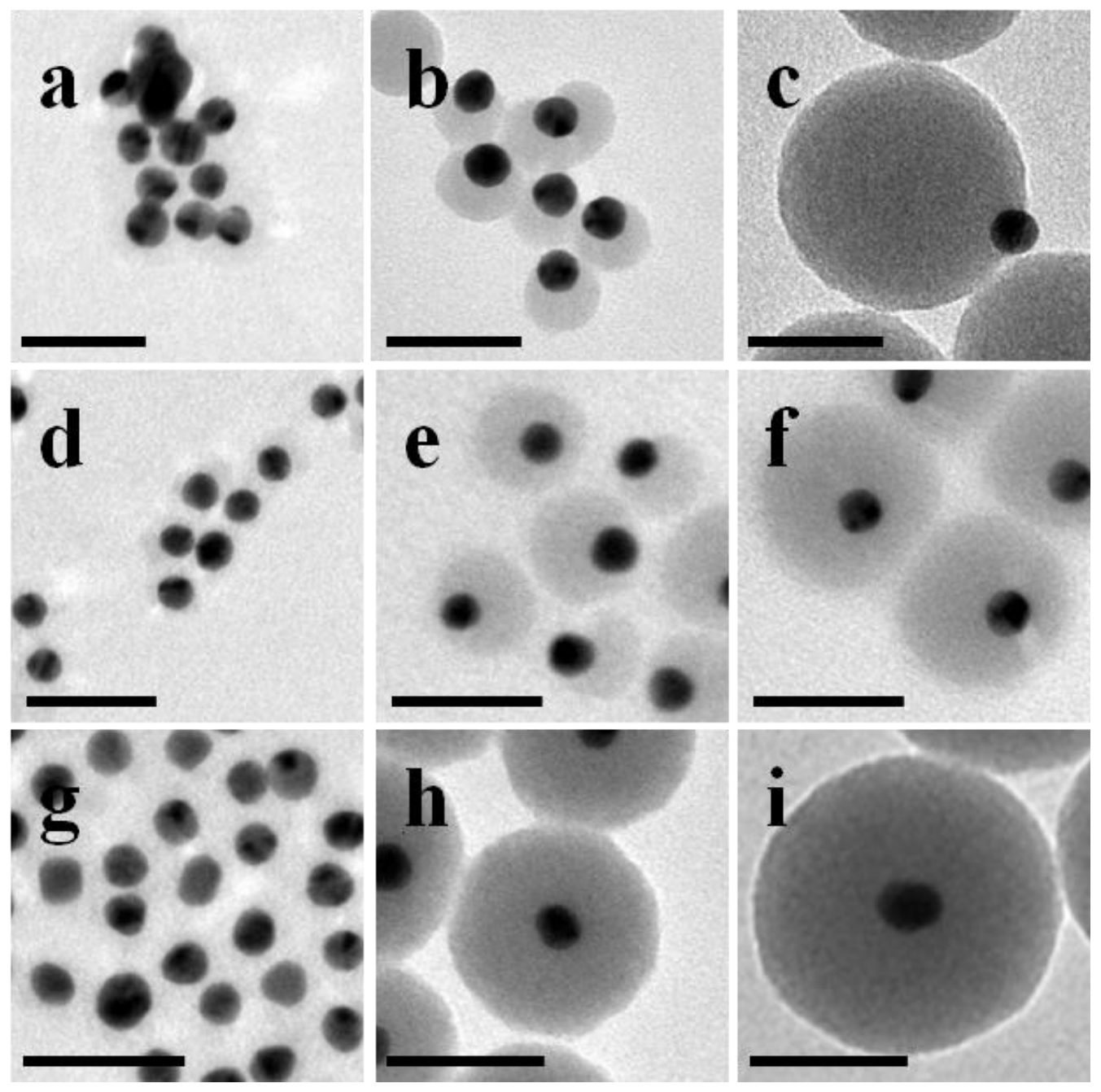

Figure 4.7. The TEM of $15 \mathrm{~nm}$ Au nanoparticles coating under different solvent ratio of $\mathrm{H}_{2} \mathrm{O} / \mathrm{IPA}$ : (a-c) 0.2, (d-f) 0.4, (g-i) 0.6 in Stöber system. The intermediates was taken out at different times during the synthesis of: (a) 30 min, (b) $40 \mathrm{~min}$, (c) 60 min Janus nanoparticles; (d) $30 \mathrm{~min}$, (e) 40min, (f) 60 min eccentric nanoparticles; (g) $30 \mathrm{~min}$, (h) 40min, (i) 60 min concentric nanoparticles. Scale bar: 100nm.

For the silica coating procedure TEOS, ammonia, and nanoparticle concentration were controlled the same value in different experiments here. Au nanoparticles were purified first, and then dispersed in water/IPA mixture drop 
by drop under vigorous stirring to avoid heavy aggregation. $10 \mu \mathrm{L}$ of 4-MBA $(2 \mathrm{mM}$, in ethanol) was added into the mixture as ligand for silica coating. Then $2 \mu \mathrm{L}$ of pure TEOS was added into the vial followed by stirring for 1 minute. Finally $40 \mu \mathrm{L}$ of ammonia (28\%) was added into the vial as catalyst for the silica coating with vigorous stirring.

As shown in figure 4.7, with the solvent ratio (water/IPA) increase in the Stöber system, the silica shell morphology is different. At the lower solvent ratio of water/IPA the ion doping inside silica is heavier which means that there is higher interfacial energy between silica and Au surface. So most of the products are eccentric or Janus nanoparticles; however, at the higher solvent ratio of water/IPA the ion doping inside silica is less which means that there is lower interfacial energy between silica shell and Au surface, and the concentric core-shell nanoparticles are the uniform product. All these trends are highly consistent with the result in reverse emulsion system. It is clear to prove our hypothesis: different solvent ratio of water/IPA gives different kinds of 'silica' because of different level of ion doping inside silica nanoparticles, and the different 'silica' has different interfacial energy value to Au surface which will affect the silica shell morphology from concentric, to eccentric and Janus nanoparticles.

All the experimental data from Stöber method, reverse emulsion method, and pure silica synthesis are consistent with our hypothesis. This understanding 
helped us to control the ion doping inside silica which now gives different kinds of core-shell nanostructures, and Janus nanoparticles.

\subsubsection{Salt addition in reverse emulsion synthesis}

Another better control of the ion doping inside silica is salt addition. We can control the salt concentration and then affect the ion doping level during the silica nucleation and deposition. On one hand, tuning the salt concentration can help to control ion doping inside silica which would give similar trend as that of solvent ratio tuning. On the other hand, the salt addition can help to rule out the ligand effect hypothesis. It is possible that solvent ratio could change the nanoparticle surface ligand solubility and then the silica coating. The solvent ratio was not changed after we use salt concentration to control the ion doping without using any IPA in the aqueous in the reverse emulsion.

What we did is $\mathrm{Fe}_{3} \mathrm{O}_{4}$ nanoparticles were used as seeds in the reverse emulsion system. Firstly $\mathrm{Fe}_{3} \mathrm{O}_{4}$ nanoparticle was dispersed inside the cyclohexane (1.5 $\mathrm{mL}$ ) by vortex to give a uniform transparent solution. Then $150 \mu \mathrm{L}$ of surfactant Igepal CO-520 was added into cyclohexane by vortex to dissolve the surfactant. Then the water and ammonia mixture $(30 \mu \mathrm{L}$ water, and $20 \mu \mathrm{L}$ ammonia) was added into the vial by vigorous vortex to give a stable transparent emulsion solution. Finally, $2 \mu \mathrm{L}$ of TEOS was added inside the vial and incubated the whole mixture for 8 hours. It is very easy to check the stability of the reverse 


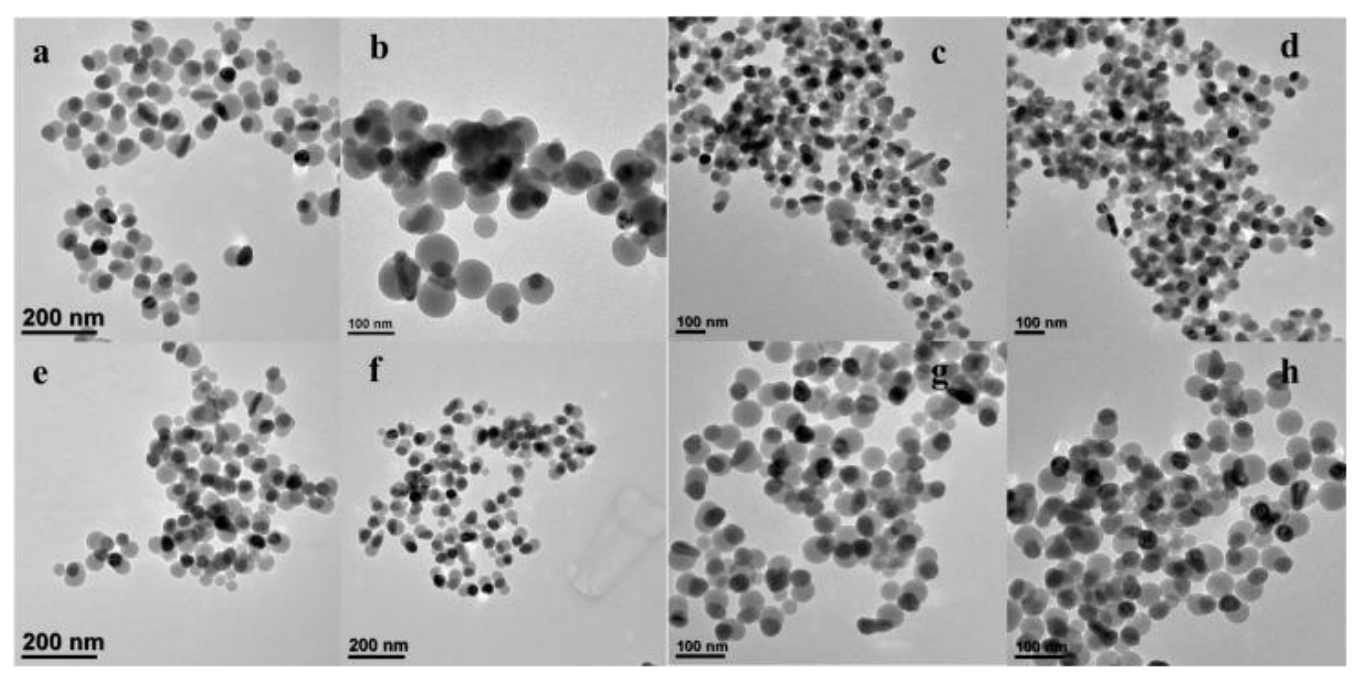

Figure 4.8. TEM images of iron oxide Janus NPs synthesis in different kinds of salt solution (a) $\mathrm{NaCl}$ (b) $\mathrm{NaNO}_{3}$, (c) $\mathrm{Na}_{2} \mathrm{SO}_{4}$ and (d) $\mathrm{CH}_{3} \mathrm{COONa}$ (e) $\mathrm{KCl}$ (f) $\mathrm{KNO}_{3}$, (g) $\mathrm{K}_{2} \mathrm{SO}_{4}$ and (h) $\mathrm{CH}_{3} \mathrm{COOK}$. The salt molar concentration was the same (10 $\mathrm{mM})$ for all the above samples.

emulsion by light transparent. In our system the droplet is very stable even more than 24 hours. Here we need to point out there is no IPA addition in the reverse emulsion system.

According to the TEM image in figure 4.8 , it is clear to see with the salt addition we can get Janus nanoparticles instead of concentric nanoparticles. With using different kinds of salt we still find the general Janus nanoparticles which help to rule out a special ion effect. Here it is a good proof of ion doping mechanism: with the addition of salt such as $\mathrm{KCl}, \mathrm{NaCl}$, or $\mathrm{NaNO}_{3}$ the ion doping inside silica will be heavier compared to the sample without any salt there. The higher level of ion doping inside silica makes a different kind of 


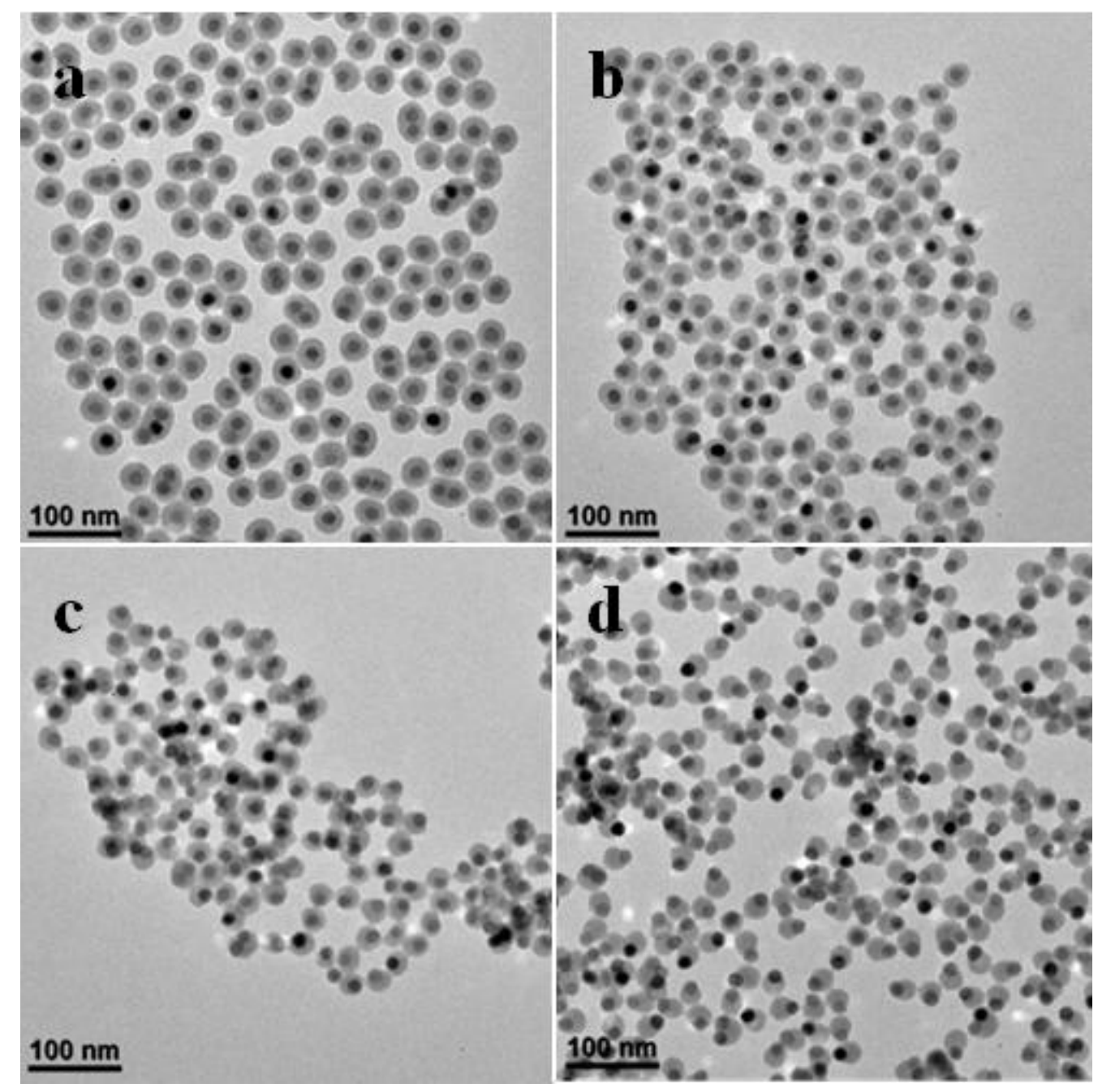

Figure 4.9. TEM images of iron oxide Janus NPs synthesis in different $\mathrm{KCl}$

concentration [C] at (a) 0, (b) $2 \mathrm{mM}$, (c) $6 \mathrm{mM}$, and (d) $10 \mathrm{mM}$ in the aqueous phase of reverse emulsion.

'silica' which shows higher interfacial energy to the Au surface. The interfacial energy increase leads to the partial encapsulation instead of full encapsulation.

In order to test our hypothesis in depth we try to tune the salt $(\mathrm{KCl})$ concentration in the aqueous to trap the shell morphology relationship with the salt concentration (ion doping). So what we did is using $\mathrm{KCl}$ solution in the aqueous solution not the pure DI water, and we change the $\mathrm{KCl}$ concentration 
from 0 to $20 \mathrm{mg} / \mathrm{mL}, 40 \mathrm{mg} / \mathrm{mL}$, and $80 \mathrm{mg} / \mathrm{mL}$ while all the other parameters are the same as the previous full encapsulation experiment condition.

As shown in figure 4.9, it is clear to see the silica shell morphologies difference under different $\mathrm{KCl}$ concentration in the aqueous phase of reverse emulsion system. In the lower $\mathrm{KCl}$ concentration the ion doping inside silica $\left(\mathrm{K}^{+}\right.$doped silica) is lower which means that the interfacial energy between 'silica' and nanoparticle surface is lower, and the core-shell nanoparticle was collected. With the increasing of the $\mathrm{KCl}$ concentration the ion doping level inside 'silica' is changed from lower to higher, which means that the interfacial energy between 'silica' shell and nanoparticle surface are also changed from lower to higher. As a result the 'silica' shell will be changed from concentric to eccentric and Janus according to the interfacial energy increase.

Here we need to point out the salt addition control experiments are difficult to conduct in the Stöber system. The reason is as the follows: firstly, the ions $\left(\mathrm{K}^{+}\right.$, $\mathrm{Na}^{+}$) addition could lead to nanoparticle aggregation which will affect the silica coating; secondly, the ions addition could lead to heavy aggregation of silica nanoparticles because of charge repulsion disappear there. However, reverse emulsion can help to avoid the aggregation problem by phase segregation, and give us perfect nanoparticles there. All the control experiments of salt concentration tuning can prove the hypothesis that ion doping difference inside silica can adjust the interfacial energy between silica and nanoparticle, which can 
help us to control the full encapsulation or partial encapsulation. This series control is easy, fast, and effective to get novel nanostructures.

\subsubsection{Generality for Janus synthesis}
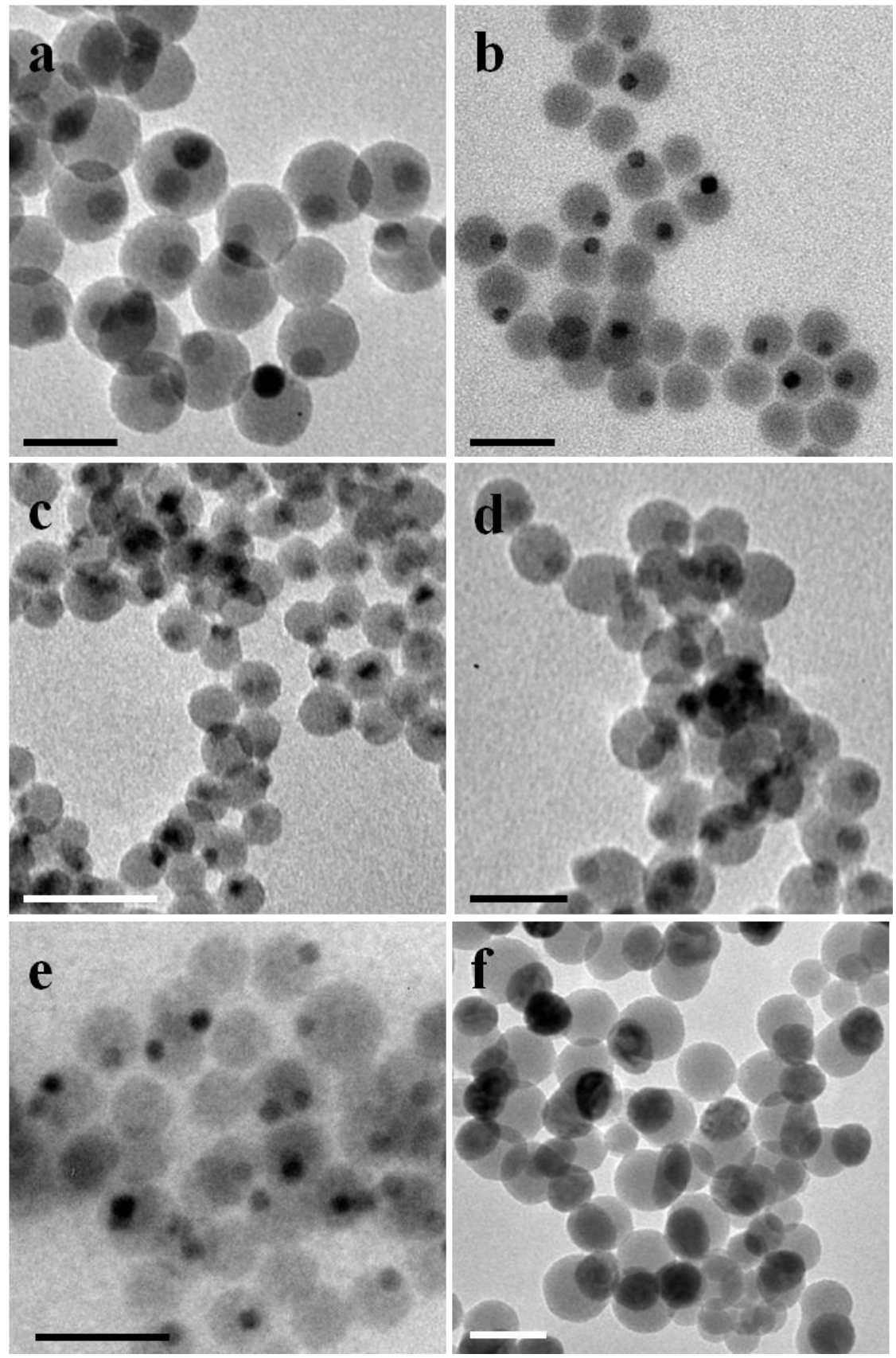

Figure 4.10.TEM images of (a) $20 \mathrm{~nm} \mathrm{Fe}_{3} \mathrm{O}_{4} @ \mathrm{SiO}_{2}$ Janus NPs (b) $12 \mathrm{~nm}$

$\mathrm{Fe}_{2} \mathrm{O}_{3} @ \mathrm{SiO}_{2}$ Janus $\mathrm{NPs}$ (c) 10 nm $\mathrm{CdSe} @ \mathrm{SiO}_{2}$ Janus NPs (d) 15 nm 
$\mathrm{CoFe}_{2} \mathrm{O}_{4} @ \mathrm{SiO}_{2}$ Janus NPs, (e) 10 nm CdS-SiO 2 Janus NPs (f) 40 nm $\mathrm{Fe}_{3} \mathrm{O}_{4} \mathrm{NPs}$ $@ \mathrm{SiO}_{2}$ Janus synthesized in reverse emulsion. Scale bar: 100nm.

In order to broad our method to different kinds of nanoparticles we have used many different kinds of nanoparticles in the reverse emulsion method. All the procedure (concentration, solvent ratio) keeps similar while seed is different.

It is clear to see from figure 4.10, all the samples give uniform Janus nanoparticles which is similar to iron oxide encapsulation. By tuning the solvent ratio or the salt $(\mathrm{KCl}, \mathrm{NaCl})$ concentration we also can change the encapsulation from core-shell nanoparticle to Janus nanoparticle. It is meaningful to the future application for nanostructure design and synthesis based on our methodology.

\subsubsection{Design nanostructure by Janus fabrication}

The Janus nanoparticles synthesized in our system can be applied to further fabrication. The Janus nanoparticles there have two different domains which show different properties which are critical to its further fabrication application in different systems. Here we show an example of $\mathrm{Fe}_{3} \mathrm{O}_{4}-\mathrm{SiO}_{2}$ Janus further fabrication using the Janus nanoparticle as a unit. We did two kinds of fabrication, the polystyrene-block-poly(acrylic acid) $\mathrm{PS}_{154}$-b-PAA 49 (PSPAA) coating control; and the silica coating of $\mathrm{Fe}_{3} \mathrm{O}_{4}-\mathrm{SiO}_{2}$ Janus nanoparticle under 

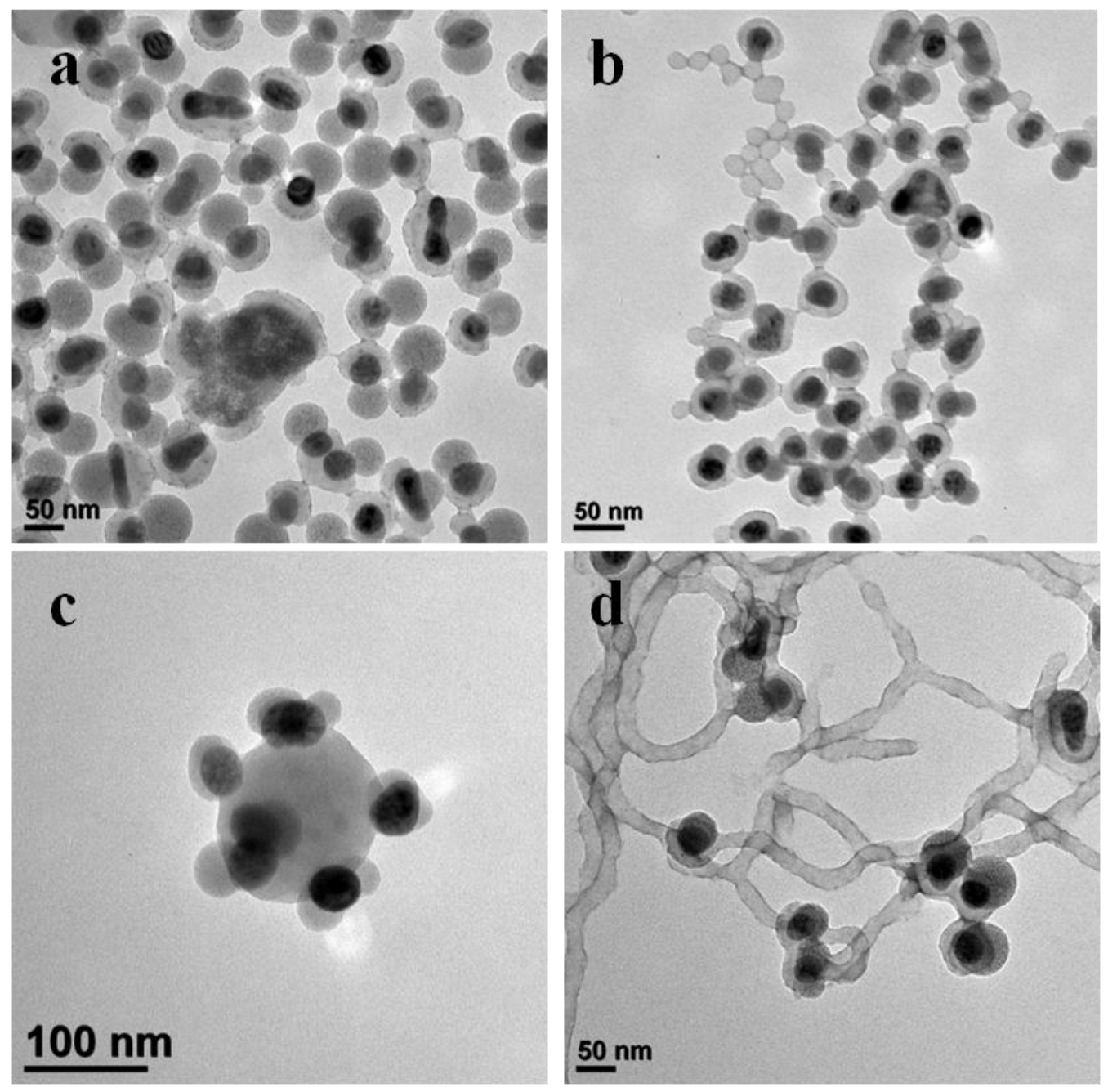

Figure 4.11. TEM images of iron oxide-silica Janus coating PSPAA in different conditions: (a-b) PSPAA directly coating, (c) $\mathrm{NaCl}$ present, (d) $\mathrm{HCl}$ present and coated by $\mathrm{PS}_{144}-\mathrm{PAA}_{22}$. (f) under magnetic field linear coating.

magnetic field fabrication. $\mathrm{Fe}_{3} \mathrm{O}_{4}-\mathrm{SiO}_{2}$ Janus nanoparticles were purified 3 times to remove the surfactants and ligands on the surface before applied. Then $\mathrm{Fe}_{3} \mathrm{O}_{4}-\mathrm{SiO}_{2}$ Janus nanoparticles were dispersed in DMF solution after citrate acid was added. The mixture was heated at $90{ }^{\circ} \mathrm{C}$ for 1 hour to do ligand exchange. The $\mathrm{Fe}_{3} \mathrm{O}_{4}-\mathrm{SiO}_{2}$ Janus nanoparticle will be amphiphilic after the ligand exchange, which means that the $\mathrm{Fe}_{3} \mathrm{O}_{4}$ domain will be hydrophobic while the 
$\mathrm{SiO}_{2}$ domain will be hydrophilic. Then we tried to coating the $\mathrm{Fe}_{3} \mathrm{O}_{4}-\mathrm{SiO}_{2}$ Janus nanoparticle by polystyrene-block-poly(acrylic acid) $\mathrm{PS}_{154}$-b-PAA 49 (PSPAA).

The coating procedure is similar as reported there. As shown in figure 4.11, it is interested to see some different kinds of novel structures were synthesized there. The flower like core-shell structure of PSPAA-Fe $\mathrm{O}_{4}-\mathrm{SiO}_{2}$ was synthesized while coating PSPAA directly; the basketball like structure of $\mathrm{Fe}_{3} \mathrm{O}_{4}-\mathrm{SiO}_{2}$ was synthesized while $\mathrm{NaCl}$ was added during the PSPAA coating procedure; the nanobelt like structure of PSPAA-Fe $\mathrm{O}_{4}-\mathrm{SiO}_{2}$ was collected while $\mathrm{HCl}$ was added during the PSPAA coating. All these novel structures were never reported and the method is simple, effective.

The PSPAA coating mechanism and control details will be discussed in chapter 5 and chapter 6.

The second fabrication is conducted under magnetic field. What we did is coating another layer of silica on the $\mathrm{Fe}_{3} \mathrm{O}_{4}-\mathrm{SiO}_{2}$ Janus nanoparticles. The Janus nanoparticles were dispersed in water/IPA mixture as Stöber reported method, and then the whole encapsulated solution was located under magnetic field during the silica coating process (6-8 hours). It is clear to see the uniformly dispersed nanoparticles will be lightly aggregated on the vial surface under the magnetic field after incubation during silica coating.

As shown in figure 4.12, it is clear to see that the linear aggregation of Janus nanoparticle happened during the silica coating. Which means that the Janus 


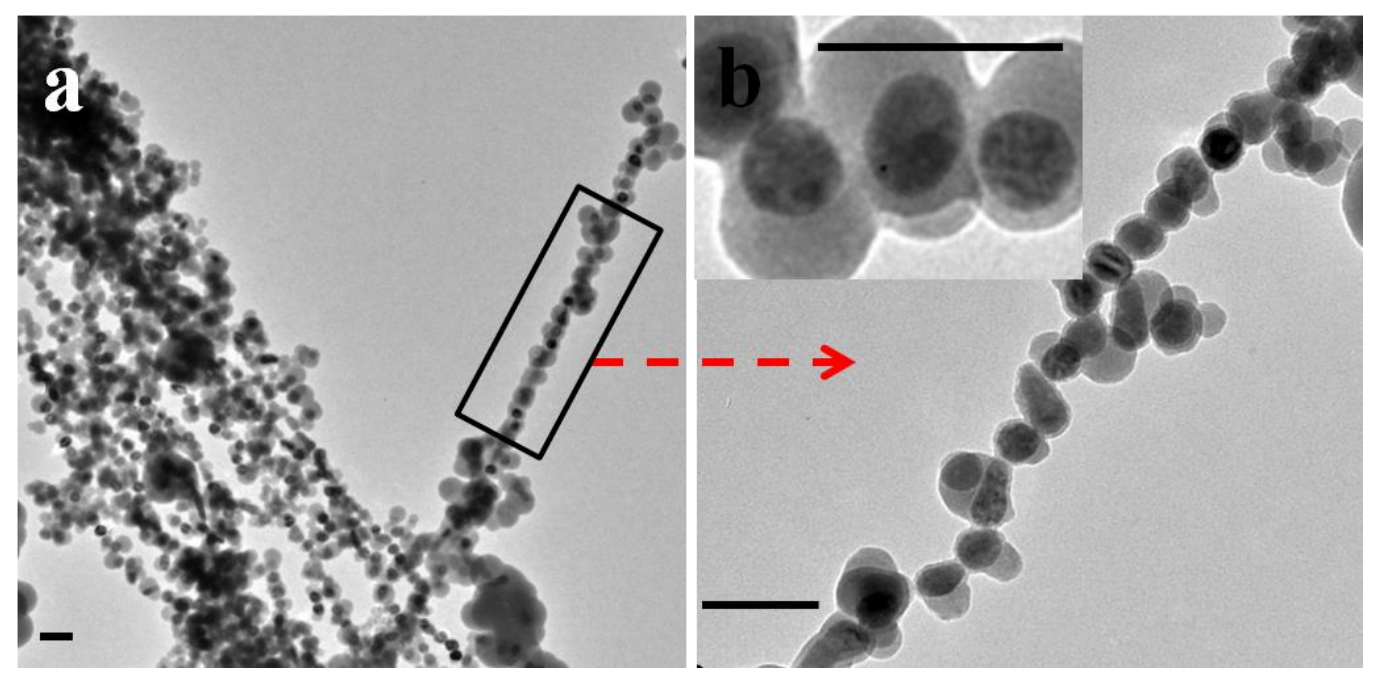

Figure 4.12. TEM images of iron oxide-silica Janus coating silica under the magnetic field. (a) low MAG TEM image of Janus linear aggregation in magnetic field after coated by silica, (b) high MAG image of Janus chains. Scale bar: $150 \mathrm{~nm}$.

nanoparticles were linear aggregated because of magnetic dipole while they were located under magnetic field. Another linear aggregation reason is because of its Janus structure properties, which means that only the $\mathrm{Fe}_{3} \mathrm{O}_{4}$ surface could touch in linear direction while the $\mathrm{SiO}_{2}$ domain helps to prohibit heavy aggregation.

With the above fabrication control it is clear that our Janus nanoparticle is useful in future application, especially in the novel structure design, the magnetic induced Janus fabrication, as well as the other second round encapsulation. Another point is that this method is very easy to upscale and very simple to control from the shell thickness to the core size, and core numbers. 


\subsection{Conclusion}

In summary, we exploited an unconventional assembly system in reverse microemulsion by controlling the solvent ratio in the aqueous phase. Adjusting solvent ratio can help to tune the interfacial energy between the nanoparticles and the silica shell, the silica precursor hydrolysis behavior, as well as silica shell expansion. Under this control, many different kinds of hydrophobic nanoparticles can be coated which gives concentric, eccentric, or Janus nanoparticles by tuning the solvent ratio in aqueous phase. This new method opens doors to Janus nanoparticle designs, the assembly of Janus nanoparticle to complex nanostructures, as well as Janus nanoparticles used for applications. 


\subsection{Bibliography}

(1) Hong, L.; Cacciuto, A.; Luijten, E.; Granick, S. Langmuir : the ACS journal of surfaces and colloids 2008, 24, 621.

(2) Hu, J.; Zhou, S.; Sun, Y.; Fang, X.; Wu, L. Chemical Society Reviews 2012, $41,4356$.

(3) Zhao, N.; Gao, M. Advanced Materials 2009, 21, 184.

(4) Leroueil, P. R.; Hong, S.; Mecke, A.; Baker, J. R.; Orr, B. G.; Banaszak Holl, M. M. Accounts of Chemical Research 2007, 40, 335.

(5) Chen, Q.; Bae, S. C.; Granick, S. Nature 2011, 469, 381.

(6) Yan, J.; Bloom, M.; Bae, S. C.; Luijten, E.; Granick, S. Nature 2012, 491, 578.

(7) Synytska, A.; Isa, L. ChemPlusChem 2014, 79, 656.

(8) Wu, B.; Tang, S.; Chen, M.; Zheng, N. Chem. Commun. 2013, 50, 174.

(9) Chen, T.; Chen, G.; Xing, S.; Wu, T.; Chen, H. Chemistry of Materials 2010, $22,3826$.

(10) Chen, T.; Yang, M.; Wang, X.; Tan, L. H.; Chen, H. J. Am. Chem. Soc. 2008, $130,11858$.

(11) Ohnuma, A.; Cho, E. C.; Camargo, P. H. C.; Au, L.; Ohtani, B.; Xia, Y. J. Am. Chem. Soc. 2009, 131, 1352.

(12) Gu, H.; Yang, Z.; Gao, J.; Chang, C. K.; Xu, B. J. Am. Chem. Soc. 2005, 127, 34. 
(13) Haruta, M. CATTECH 2002, 6, 102.

(14) Xu, Z.; Hou, Y.; Sun, S. J. Am. Chem. Soc. 2007, 129, 8698.

(15) Chen, Y.; Washburn, J. Physical review letters 1996, 77, 4046.

(16) Carbone, L.; Cozzoli, P. D. Nano Today 2010, 5, 449.

(17) Seo, D.; Yoo, C. I.; Jung, J.; Song, H. J. Am. Chem. Soc. 2008, 130, 2940.

(18) Lin, Z.-H.; Lin, Y.-W.; Lee, K.-H.; Chang, H.-T. Journal of materials chemistry 2008, 18, 2569.

(19) Song, L.; Qiao, Y.; Liu, Z.; Li, Y. Polymer Chemistry 2015, 6, 896.

(20) Santra, S.; Tapec, R.; Theodoropoulou, N.; Dobson, J.; Hebard, A.; Tan, W. Langmuir : the ACS journal of surfaces and colloids 2001, 17, 2900.

(21) Laurent, S.; Forge, D.; Port, M.; Roch, A.; Robic, C.; Vander Elst, L.; Muller, R. N. Chem. Rev. 2008, 108, 2064.

(22) Wang, Y.; He, J.; Liu, C.; Chong, W. H.; Chen, H. Angewandte Chemie International Edition 2015, 54, 2022.

(23) Torza, S.; Mason, S. G. J. Colloid Interface Sci. 1970, 33, 67.

(24) Wang, H.; Chen, L.; Feng, Y.; Chen, H. Accounts of Chemical Research 2013, 46, 1636.

(25) Sun, H.; He, J.; Wang, J.; Zhang, S.-Y.; Liu, C.; Sritharan, T.; Mhaisalkar, S.; Han, M.-Y.; Wang, D.; Chen, H. J. Am. Chem. Soc. 2013, 135, 9099.

(26) Ding, Y.; Gao, P. X.; Wang, Z. L. J. Am. Chem. Soc. 2004, 126, 2066.

(27) Peng, Z.; Yang, H. Nano Today 2009, 4, 143. 
(28) Li, X.; Zhou, L.; Wei, Y.; El-Toni, A. M.; Zhang, F.; Zhao, D. J. Am. Chem. Soc. 2015.

(29) Li, X.; Zhou, L.; Wei, Y.; El-Toni, A. M.; Zhang, F.; Zhao, D. J. Am. Chem. Soc. 2014, 136, 15086.

(30) Feng, Y.; He, J.; Wang, H.; Tay, Y. Y.; Sun, H.; Zhu, L.; Chen, H. J. Am. Chem. Soc. 2012, 134, 2004.

(31) Mishin, Y.; Mehl, M.; Papaconstantopoulos, D.; Voter, A.; Kress, J. Physical Review B 2001, 63, 224106.

(32) Song, X.; Ding, T.; Yao, L.; Lin, M.; Tan, L. S. R.; liu, C.; Yu, L.; Lou, X. W. D.; Chen, H. small 2015, In revision.

(33) Muhr, V.; Wilhelm, S.; Hirsch, T.; Wolfbeis, O. S. Accounts of Chemical Research 2014, 47, 3481.

(34) Selvan, S. T. Biointerphases 2010, 5, FA110.

(35) Teruoki Tago, V.; Hatsuta, T.; Miyajima, K.; Kishida, M.; Tashiro, S.; Wakabayashi, K. J. Am. Ceram. Soc 2002, 85, 2188.

(36) Santra, S.; Tapec, R.; Theodoropoulou, N.; Dobson, J.; Hebard, A.; Tan, W. Langmuir : the ACS journal of surfaces and colloids 2001, 17, 2900.

(37) Deng, Y.; Qi, D.; Deng, C.; Zhang, X.; Zhao, D. J. Am. Chem. Soc. 2008, $130,28$.

(38) Wang, Y.; Xu, J.; Wang, Y. W.; Chen, H. Y. Chem. Soc. Rev. 2013, 42, 2930.

(39) Bonn, D.; Eggers, J.; Indekeu, J.; Meunier, J.; Rolley, E. Reviews of modern 
physics 2009, 81, 739.

(40) Li, Z.; Wang, Y.; Kozbial, A.; Shenoy, G.; Zhou, F.; McGinley, R.; Ireland, P.; Morganstein, B.; Kunkel, A.; Surwade, S. P. Nature materials 2013, 12, 925.

(41) Yuan, Y.; Lee, T. R. In Surface science techniques; Springer: 2013, p 3.

(42) Narita, A.; Naka, K.; Chujo, Y. Colloids and Surfaces A: Physicochemical and Engineering Aspects 2009, 336, 46. 


\section{Chapter 5 Synthesis and Mechanism Investigation of the Metal@PSPAA Nanoparticles}

\subsection{Introduction}

It is well known that coating NPs with a shell has several advantages. The encapsulated NPs with their surface ligands are protected so that their chemical and colloidal stability do not depend on the excess ligands in the media. Preventing ligands from dissociation and exchange is of importance for applications such as surface enhanced Raman scattering (SERS), ${ }^{1}$ where the Raman signals are derived from the surface ligands. To render the encapsulated NPs stable in aqueous solution, typically polymers, proteins, or inorganic materials (e.g., silica) have been attached to the NP surface by either chemical reaction or deposition. ${ }^{2}$ While the water-facing surface of these shell materials must has charge, polar, or H-bonding functionalities for water solubility, their interface with the embedded NPs is also important in order for the encapsulation to work in the first place. For example, it is difficult to chemically link proteins to non-polar nanoparticle surface; surface initiators are necessary for grafting polymers; for silica encapsulation, NPs need to be functionalized with vitrophilic compounds in order for silica adsorption. ${ }^{3-7}$ Typically, if the NP-shell interface has a high surface tension, incomplete encapsulation will result. Since the core-shell interactions are often highly specific to both core and shell materials, 
most encapsulation methods have to be specifically developed and fine-tuned.

Photon upconversion is promising for applications such as biological imaging, data storage or solar cells. Here, we have investigated upconversion processes in a broad range of gadolinium-based nanoparticles of varying composition. We show that by rational design of a core-shell structure with a set of lanthanide ions incorporated into separated layers at precisely defined concentrations, efficient upconversion emission can be realized through gadolinium sublattice-mediated energy migration for a wide range of lanthanide activators without long-lived intermediary energy states. Furthermore, the use of the core-shell structure allows the elimination of deleterious cross-relaxation. This effect enables fine-tuning of upconversion emission through trapping of the migrating energy by the activators. Indeed, the findings described here suggest a general approach to constructing a new class of luminescent materials with tunable upconversion emissions by controlled manipulation of energy transfer within a nanoscopic region.

The vision to engineer nanodevices from building blocks with controllable self-assembly and respond to give designated functions upon stimuli is no doubt challenging and difficult. ${ }^{1}$ Colloidal self-assembly method holds great potential for large-scale synthesis at low expense of energy as compared to methods that assemble molecular by manipulating building blocks one after another. The development of the colloidal method, however, requires careful and intelligent 
design of the building blocks as numerous degrees of freedom like isotropic nanoparticles generally form highly repetitive, lattice like structures or random aggregates upon self-assembly due to their identical surfaces throughout. It has been demonstrated if one can precisely control the localisation of surface functionalities (i.e. ligands, or polymer shell) on the nanoparticles (NPs), a plethora of self-assembled structures (e.g., chains, sheets, rings, twisted and even staircase structures) would be possible..$^{2-4}$ Despite numerous simulations and modelling for this type of NPs, just a handful reported the synthesis of patchy NPs through colloidal methods. ${ }^{5}$ The progress is largely disabled by the current characterization methods which do not have the detection limit and resolution sufficient at the nanoscale. With the absence of such technologies, the forces and interaction between ligands that govern the assembly on nanostructures are still obscure.

Our previous report on the synthesis of anisotropic NPs utilises the binding competition between hydrophobic ( $\mathrm{L}_{\mathrm{A}}$, e.g. 1) and hydrophilic ligands $\left(\mathrm{L}_{\mathrm{B}}\right.$, e.g. 3) on NPs to give selective attachment of amphiphilic diblock copolymer polystyrene-b-poly(acrylic acid) $\left(\mathrm{PS}_{144} \mathrm{PAA}_{28}\right)$ (aniso-(1+3-AuNP)@PSPAA). ${ }^{6}$ The generality of this method has been demonstrated for a variety of cores, polymers, and ligand combinations. ${ }^{7}$ The polymer which have a general thickness of about $14 \mathrm{~nm}$ can be distinctly observed under TEM. The advantage of this observable shell permits a simple qualitative measurement of the 
wettability of the NP surface.

Here we report a detailed mechanistic study on the anisotropic encapsulation of metal NPs that we have previously reported. We address the conformation of ligand shell on the metal surface and the thermodynamically avoured polymer assembly on the ligand coated metal core. The partial polymer attachment on NPs allows selective detection of the underlying ligand system on the metal surface using SERS and TEM studies. By understanding the underlying mechanisms, a new platform for the synthesis of anisotropic NPs with the ability to control the patches in precise manner would be created.

\subsection{Materials and methods}

\subsubsection{Materials}

All chemical reagents were used without further purification. Hydrogen tetrachloroaurate (III) hydrate $99.9 \%$ (metal basis $\mathrm{Au} 49 \%$ ) and 2-dipalmitoyl-sn-glycero-3-phosphothioethanol (sodium salt) (1) were purchased from Avanti Polar Lipids; 4 -mercaptobenzoic acid 90\% (tech) (2) was purchased from Sigma Aldrich; dimthylamine (3) were purchased from Alfa Aesar; amphiphilic diblock copolymers polystyrene-block-poly(acrylic acid) $\mathrm{PS}_{144}$-b-PAA $28, \mathrm{Mn}=15000$ for the PS block and $\mathrm{Mn}=1600$ for the PAA block, Mw / Mn = 1.11 was purchased from Polymer Source Inc.; 200 mesh copper specimen grids with formvar/carbon support film (referred to as TEM grids in text) was purchased from Electron Microscopy Sciences. Ultrapure water with 
resistivity $>18 \mathrm{M} \Omega \cdot \mathrm{cm}-1$ was used for all experiments. TEM grids were treated with oxygen plasma in a Harrick@ plasma cleaner/sterilizer for 1 min to improve the surface hydrophilicity. The product solution was diluted $(300 \mu \mathrm{L}$ solution added to $1200 \mu \mathrm{L}$ DI water) and then centrifuged at designed speed (depend on size of NPs) for 30 min to remove the empty micelles. Then a sample solution was carefully mixed with stain solution $\left(\left[\left(\mathrm{NH}_{4}\right) \mathrm{Mo}_{7} \mathrm{O}_{24}\right]=6.8 \mathrm{mM}\right)$ on the surface of a plastic Petri dish, the hydrophilic face of the TEM grid was then placed in contact with the sample solution. A filter paper was used to wick off the excess solution on the TEM grid, which was then dried in air for $5 \mathrm{~min}$. TEM images were collected from a JEM-1400 Transmission Electron Microscopy (JEOL) operated at $120 \mathrm{kV}$. UV-vis spectra were collected on a Cary $100 \mathrm{UV}$-vis spectrophotometer.

\subsubsection{Methods}

Preparation of TEM samples. TEM grids were treated by oxygen plasma in a Harrick $^{\circledR}$ plasma cleaner/sterilizer for $1 \mathrm{~min}$ to improve the surface hydrophilicity. A sample solution was dropped on the hydrophilic surface of the TEM grid. Finally, a filter paper was used to wick off the excess solution on the TEM grid, and the grid was dried in air for $30 \mathrm{~min}$.

Characterization. TEM images were collected on a JEM-1400 (JEOL) Transmission Electron Microscope operated at $100 \mathrm{kV}$. UV-Vis spectra were collected from a Cary 100 UV-vis spectrophotometer. Surface-enhanced Raman 
scattering (SERS) spectra were collected from sample solutions using $8 \mathrm{~mL}$ glass vials and recorded using a PeakSeeker Pro Spectrometer (Raman System Inc.) using a red laser ( $\lambda=785 \mathrm{~nm}$ ) at $290 \mathrm{~mW}$. Integration time was set at 10 frames per second. Collected data was saved on (.csv) files and processed using Microsoft Excel. Graphs of intensity (a.u.) against Raman shift $\left(\mathrm{cm}^{-1}\right)$ were plotted for all experiments. The decrease in SERS intensity was monitored at $1067 \mathrm{~cm}^{-1}$ corresponding to the peak intensity of the 2-napthalenethiol ligand.

Preparation of Au nanoparticles. Citrate-stabilized gold nanoparticles ( $\mathrm{d}=$ $50 \pm 5 \mathrm{~nm}$ ) was synthesized using previously reported methods. ${ }^{1,2}$ In a typical procedure, $50 \mathrm{~mL}$ of deionized water and $500 \mu \mathrm{L}$ of $\mathrm{HAuCl}_{4}(10 \mathrm{mg} / \mathrm{mL})$ was heated with reflux for $30 \mathrm{~min}$. Subsequently, $750 \mu \mathrm{L}$ of sodium citrate tribasic ( $1 \%$ weight) was added. At this point, $40 \mathrm{~nm}$ of colloidal gold seeds were formed. Next, $100 \mu \mathrm{L}$ of $\mathrm{NaOH}(6.6 \mathrm{mg} / \mathrm{mL})$ was added dropwise to the solution followed by $50 \mathrm{~mL}$ of hot boiling deionized water. Next, $500 \mu \mathrm{L}$ of citrate was quickly added, followed by $500 \mu \mathrm{L}$ of $\mathrm{HAuCl}_{4}$ and allowed to be heated at reflux. The addition of $\mathrm{NaOH}$, citrate and $\mathrm{HAuCl}_{4}$ were repeated three times. Later, $1 \mathrm{~mL}$ of citrate and $\mathrm{HAuCl}_{4}$ were added. Each cycle involved an incubation step to allow the refluxing of the solution to take place. The whole procedure involved a total of 9 cycles ( 3 addition of water, 6 non-addition of water) to form the $50 \pm 5 \mathrm{~nm}$ citrate stabilized gold. The resultant solution was cooled to room temperature and stored for further use. Note: More than one batch of AuNPs were synthesized and 
used in this study.

Preparation of the Au NPs@ PSPAA. 3 mL of citrate stabilized AuNP (50 \pm $5 \mathrm{~nm}$ ) was concentrated to $\sim 10 \mu \mathrm{L}$ via centrifugation at $3000 \mathrm{~g}$ for $10 \mathrm{~min}$. The deep red solution collected at the bottom of the eppendorf tubes was transferred into a $4 \mathrm{~mL}$ vial containing $718 \mu \mathrm{L}$ of DMF. Then, 2-napthalenethiol $(20 \mu \mathrm{L}, 3$ $\mathrm{mg} / \mathrm{mL}$ in DMF) was added into the mixture, vortexed, and incubated at $60{ }^{\circ} \mathrm{C}$ for 2 h. Subsequently, $80 \mu \mathrm{L}$ of $\mathrm{PS}_{154} \mathrm{PAA}_{49}(8 \mathrm{mg} / \mathrm{mL}$ in $\mathrm{DMF})$ and $172 \mu \mathrm{L}$ of deionised water were added into the solution. The final solution was then heated at $110{ }^{\circ} \mathrm{C}$ for $2 \mathrm{~h}$ and cooled slowly to room temperature. Here we can tune the solvent ratio (DMF/water, THF/water) while control the total volume is not changed. For some case, we can also change solvent to instead of DMF by THF, ethanol, and so on. All the other conditions remain the same while we tune the solvent here, including the total volume, the temperature, the concentration, and then preparation procedure.

Etching experiment. The as-synthesized of core-shell NPs were diluted (500 $\mu \mathrm{L}$ by $2.4 \mathrm{~mL}$ water), separated into individual eppendorf tubes, and centrifuged at $3000 \mathrm{~g}$ for $10 \mathrm{~min}$ to remove the supernatant. The red solution at the bottom of eppendorf tubes was dispersed into $1 \mathrm{~mL}$ solvent medium in an $8 \mathrm{~mL}$ vial. Subsequently, $200 \mu \mathrm{L}$ of $\mathrm{KCN}(40 \mathrm{mM})$ was added and allowed to be etched at room temperature. Etching was monitored for $90 \mathrm{~h}$. 


\subsection{Results and discussion}

The partial wettability, which is closely related to the interfacial tension or energy, could influence the interaction parameter or lattice mismatch between two solids and dictate the growth of a second solid material (e.g. hybrid inorganic-inorganic structures) where epitaxial growth only occurs when there is low lattice mismatch. ${ }^{8,9}$ The interfacial tension between two liquids in liquid-liquid hybrid systems has also been demonstrated to govern the spreading of one liquid on another immiscible liquid, resulting in the formation of hollow polymeric structures with open cavity (organic-organic systems). ${ }^{10-12}$ Inorganic-organic hybrid materials (solid-liquid) are potentially useful due to the synergy between two distinctly different materials. Owing to the large interfacial tension between the two materials, the controllable combination of inorganic and organic materials together to form anisotropic structures proved to be challenging. In our system, the attachment of polystyrene from PSPAA to AuNP was facilitated by the use of ligand shell. By changing the hydrophobicity or hydrophilicity of the ligand shell using different molecules, we could tune the

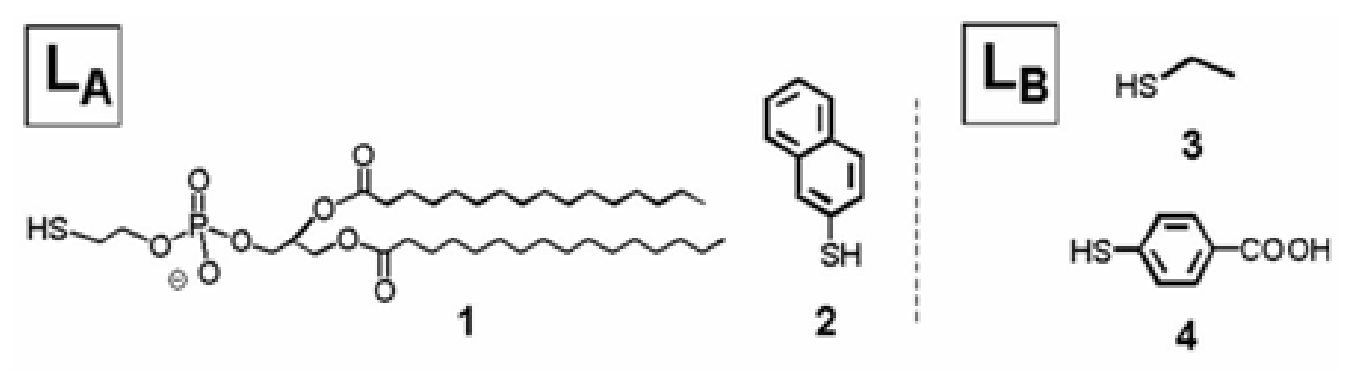

Figure 5.1. ligands used in the Au nanoparticles encapsulation. 
surface energy of the AuNP, making the interfacial tension between the metal and PS smaller or larger. As a result, tunable coverage of the polymer on the metal could be achieved (Figure. 5.1). A model which fittingly explains the tunability of these structures is based on the minimization of overall free energy of the structure, where complete wetting of the solid (1) by the liquid (2) in a solvent (3) would fulfil Equation I, where $\gamma$ is the interfacial energy term. ${ }^{13}$ Based on the expression in Equation (I), if the interfacial energy between the solid and the solvent $\left(\gamma_{13}\right)$ is higher as compared to that between the liquid and solvent $\left(\gamma_{23}\right)$ and between the solid and liquid $\left(\gamma_{12}\right)$, the liquid phase would favourably surround the solid to give a lower overall surface energy. In our system, (1) is defined as the AuNP, (2) the diblock polymer PSPAA, and (3) DMF/water mixture. Previously, Semal et al. have demonstrated the change in critical surface tension of gold surface when different amount of hydrophilic and hydrophobic alkanethiols were used. ${ }^{14}$ On the same note, we expect the interfacial energy of the AuNP with the

$$
\gamma_{13}>\gamma_{12}+\gamma_{23} \quad \text { Equation I }
$$




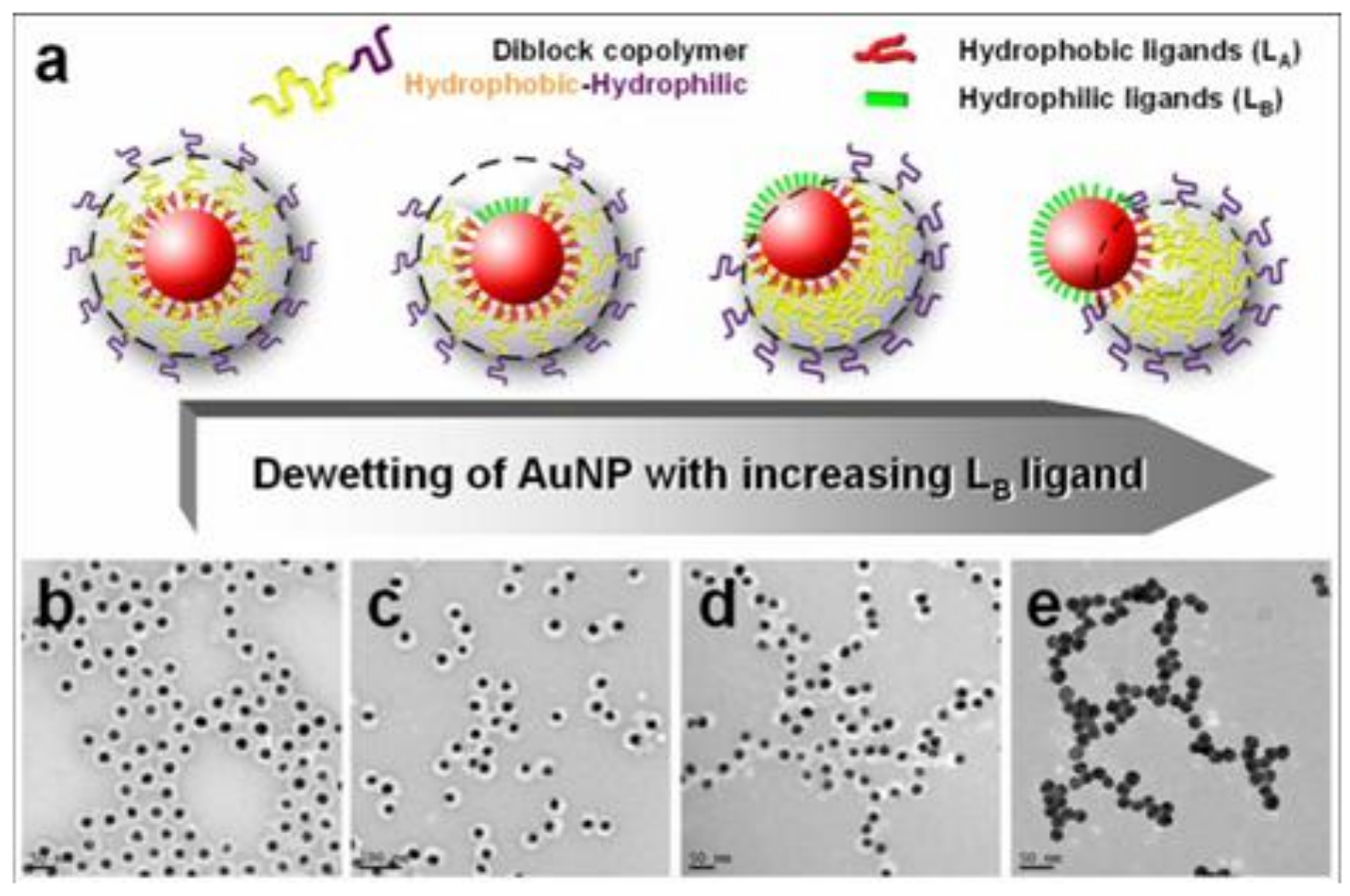

Figure 5.2. a) Schematcs demonstrating the structure of (aniso-( $\left.\left.\mathrm{L}_{\mathrm{A}}+\mathrm{L}_{\mathrm{B}}-\mathrm{AuNP}\right) @ \mathrm{PSPAA}\right)$ with decreasing PSPAA coverage with the increase of $\mathrm{L}_{\mathrm{B}}$ ligands; $\mathrm{b}$-e) Corresponding TEM images of the structures at decreasing $\mathrm{L}_{\mathrm{A}} / \mathrm{L}_{\mathrm{B}}$ ratios.

solvent or the polymer, $\gamma_{13}$ and $\gamma_{12}$, respectively, would be largely determined by the ligand shell covered AuNPs instead of the naked AuNP surface. Evidently when hydrophobic $\mathrm{L}_{\mathrm{A}}$ was the only ligand used, the unstable $\mathrm{L}_{\mathrm{A}}$-ligand shell in polar solvent caused a higher $\gamma_{13}$ and lower $\gamma_{12}$ for AuNPs, resulting in complete wetting of AuNP by the polymer (Figure. 5.2b). On the other hand, control experiments using only $\mathrm{L}_{\mathrm{B}}$ typically gave AuNPs without polymer attachment (Figure 5.2e), indicating that $\mathrm{L}_{\mathrm{B}}$-AuNP favours the solvent (lower $\gamma_{13}$ ) more than PS of the polymer (higher $\gamma_{12}$ ). The change in $\gamma_{13}$ and $\gamma_{12}$ cooperatively revert the inequality of equation (I) in agreement with dewetting of the AuNP. Both the one 
ligand systems clearly demonstrate the role of the ligand shell on the interfacial energy of AuNP which dictates complete wetting or dewetting of the metal NP.

\subsubsection{Ligand effect}

When both $\mathrm{L}_{\mathrm{A}}$ and $\mathrm{L}_{\mathrm{B}}$ mixture were used, partial wetting of the AuNPs were obtained (Figure. 5.2c,d). The Au-PS interfacial area was found to be largely dependent on the $L_{A} / L_{B}$ competition with lesser polymer coverage at higher $L_{B}$ concentration (Figure. 5.2a). During the self-assembly, the swelled PS core is capable of adjusting to equilibrium conformation that minimizes its interfacial energy $\left(\gamma_{12}+\gamma_{23}\right)$. This results in the partial encapsulation of AuNPs while the PS-water interface (outlined in Figure. 5.2) is always spheroidal, regardless of the relative position of AuNPs. In this system, the wetting or interfacial tension is controlled by the competition between the two ligands that form the ligand shell.

There are two possible arrangements of the two ligands on AuNPs that could both lead to incomplete wetting of the AuNP: (a) a phase segregated system with discrete hydrophobic LA and hydrophilic $\mathrm{L}_{\mathrm{B}}$ regions (Figure. 5.3a); or (b) a homogeneous system with $\mathrm{L}_{\mathrm{A}}$ and $\mathrm{L}_{\mathrm{B}}$ interdigitated on the surface of AuNP (Figure. 5.3b). Case (a) suggests a localised change in interfacial energy with $\mathrm{L}_{\mathrm{A}}$ regions wetted and $L_{B}$ regions dewetted, while case (b) suggests a global change in interfacial energy which is dependent on the $\mathrm{L}_{\mathrm{A}} / \mathrm{L}_{\mathrm{B}}$ ratio of the ligand shell.

The ligand organization on metal nanoparticles has long been a puzzled issue. 

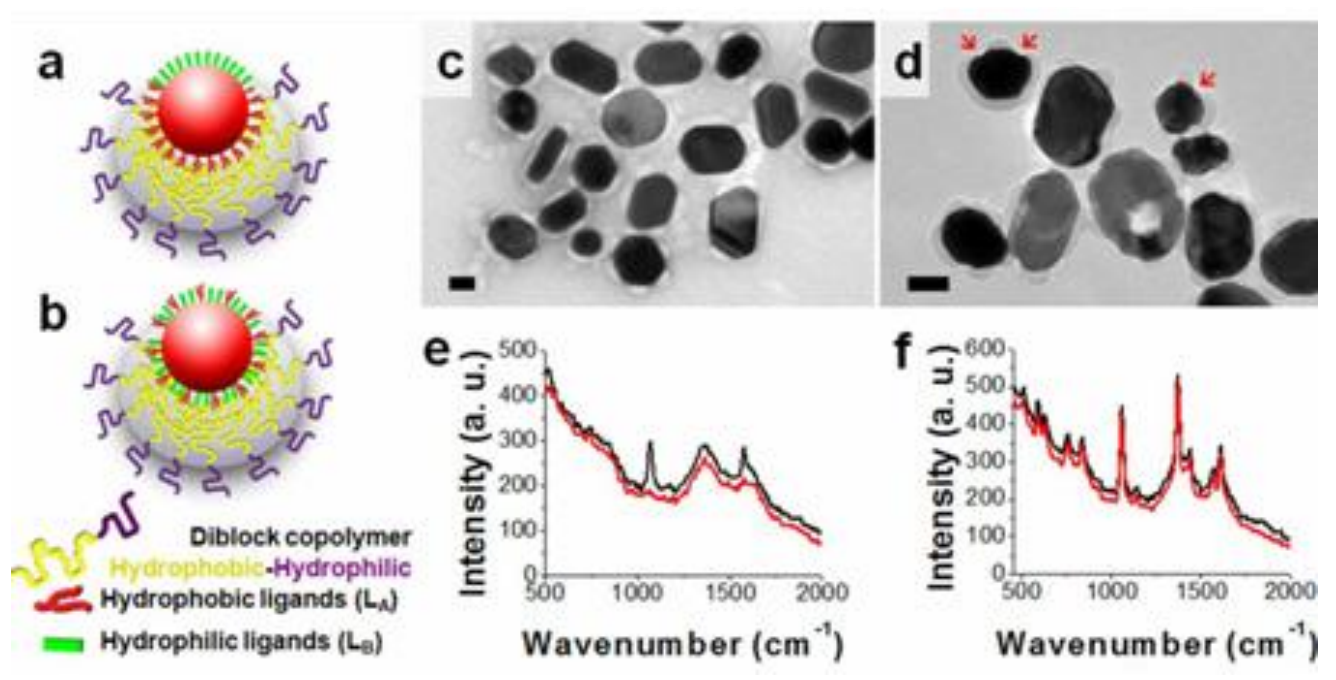

Figure 5.3. Schematics of the two possible arrangement of ligand shell on AuNP: a) phase segregated, and b) interdigitated; TEM images of ecc-(1+4-AuNP( $80 \mathrm{~nm})) @$ PSPAA c) before and d) after addition of small amount of KCN; e) Raman spectra of sample c) before (black) and d) after etching (red); f) no changes in intensity for fully encapsulated AuNP by ligand 2 before and after addition of KCN. Scale bars: $50 \mathrm{~nm}$.

Studies of ligand packing on NPs have been very limited mainly due to the size of the NPs. The highly curved surface structure of the NPs further complicates this study. ${ }^{15}$ Investigations on ligand packing on flat Au surfaces mixing hydrophobic and hydrophilic ligands using STM have found both random distribution and also disordered phase separated domains for different cases. ${ }^{16,17}$ Packing of ligands on NPs was first reported by Stellecci form of interdigitated stripped domains that ranges from 2.5 to $8.0 \mathrm{~nm}$ using STM. ${ }^{18,19}$ This nanoscale packing of mixed ligands to form segregated stripes of ligands on Au surfaces were also found to be energetically favourable based on theoretical computation and simulation. 
Electron spin resonance results from Lucarini and Pasquato also supported ligand segregation of mixed ligands with spin labels. ${ }^{22}$ (discuss UV and FT-IR result here).

In our mix ligand system, we resolved the ambiguity of ligand organisation on AuNPs by covering AuNP with Raman active ligand and using SERS to identify its presence in close proximity to the Au surface. The intensity of SERS spectra was directly correlated to the concentration of the ligand. Using as-synthesised aniso-(1+4-AuNP)@PSPAA $\left(d_{\mathrm{AuNP}}=80 \mathrm{~nm}\right.$, Figure. 5.3c $)$ with Raman active 4 as $\mathrm{L}_{\mathrm{B}}$, we detect characteristic SERS peaks of 4 at 1069 and $1580 \mathrm{~cm}^{-1}$. Previously, we have confirmed that the ligands on the exposed surface of AuNP could be exchanged by a Raman active ligand by observing the SERS of the Raman active ligand after replacement.6 The SERS signal suggests the presence of ligand closely attached to (1+4-AuNP)@PSPAA. The addition of a small amount of $\mathrm{KCN}$ was found to cause a gradual decrease in the Raman signals of $\mathbf{4}$ (Figure. 5.3e). TEM characterization of the sample immediately after the disappearance of the peaks revealed etching of Au only at areas not covered by the polymer (depressions on Au surface indicated by arrows in Figure. 5.3d). Control experiments using iso-(2-AuNP)@PSPAA showed no observable decrease in Raman intensity even after 2 days (Figure. 5.3f). These results proved that the majority of ligand 4 on AuNPs are exposed to the aqueous reactants. The evidence suggested significant ligand segregation in our system, though 
characterization at molecular details is not available to study the low-percentage mixing of the ligands. To our knowledge, this is one of few indirect evidences for ligand segregation on metal surfaces. ${ }^{23,24}$ Although we cannot rule out the possibility that the ligand segregation was a result of polymer attachment, we could identify the polymer attached regions as ligand shell regions of $\mathrm{L}_{\mathrm{A}}$ and vice versa

\subsubsection{PSPAA assembly mechanism onto Au nanoparticle}

To achieve greater extent of structural control, we have to understand the properties of the amphiphilic diblock polymer PSPAA and its assembly onto AuNP. The shell formed by PSPAA surrounding a NP symmetrically or anisotropically has not been well studied due to the dual properties of the block polymer with a defined length which cannot be assumed as a uniform material. PSPAA aggregates into micelles above its critical micelle concentration with the micelle size and morphology known to be controlled by three factors: (a) the PS interfacial energy seeks minimum surface/volume ratio and thus enlarges micelle, (b) the repulsion among crowded PAA chains favors small micelle, and (c) the thickness of PS core is limited by the physical length of PS chains and the entropic penalty for stretching them. ${ }^{25}$ The balance of the three forces led to the prevailing minimum free-energy states, where the radius $\left(r_{0}\right)$ of empty micelles and the shell thickness $\left(t_{0}\right)$ of iso-( $\left.\mathrm{L}_{\mathrm{A}}-\mathrm{AuNP}\right) @$ PSPAA reached optimal values and were thus highly uniform. ${ }^{26}$ In the unperturbed state, the polystyrene 
segment is expected to be only approximately $8.5 \mathrm{~nm}$ calculated from Equation (II) where $R_{0}$ is the unperturbed length of PSPAA, $M(\mathrm{~g} / \mathrm{mol})$ is the polystyrene block molecular weight and the value 0.067 applies to various solvents. ${ }^{27}$

Comparing this unperturbed PS length to the thickness of the PS chain encapsulating a core NP of $20 \mathrm{~nm}$ obtained from TEM, the degree of stretching was calculated to be 1.6 for the PS chain. This amount is slightly higher than what was reported previously $(0.72 \sim 1.47)$ which studied on the formation of PSPAA micelles. The additional degree of stretching is expected to be ascribed to

$$
R_{0}(\mathrm{~nm})=0.067 \times M^{1 / 2} \quad \text { Equation II }
$$

the presence of a solid core as the surface area per corona chain would be smaller due to constrain of surface curvature dictated by the metal core. This smaller surface area per corona chain results in larger repulsion between the PAA corona chains. Immediate response to counter the repulsion force is through stretching of the PS chain. In principle, a polymer shell thicker than $t_{0}$ would induce more stretching of PS chains and closer packing of the polymers, i.e., stronger PAA repulsion (thick-shell penalty), while a shell thinner than $t_{0}$ would mean high surface/volume ratio and thus high interfacial energy (thin-shell penalty)

When only $\mathrm{L}_{\mathrm{A}}$ was used in the synthesis, the $\mathrm{L}_{\mathrm{A}}$-AuNPs are always found to be in the center of PSPAA micelles with a uniform PSPAA $t_{0}$ of $14 \mathrm{~nm}$. This concentric preference can be explained by the above mentioned three forces where eccentrically positioned AuNP within the polymer shell would bring about 


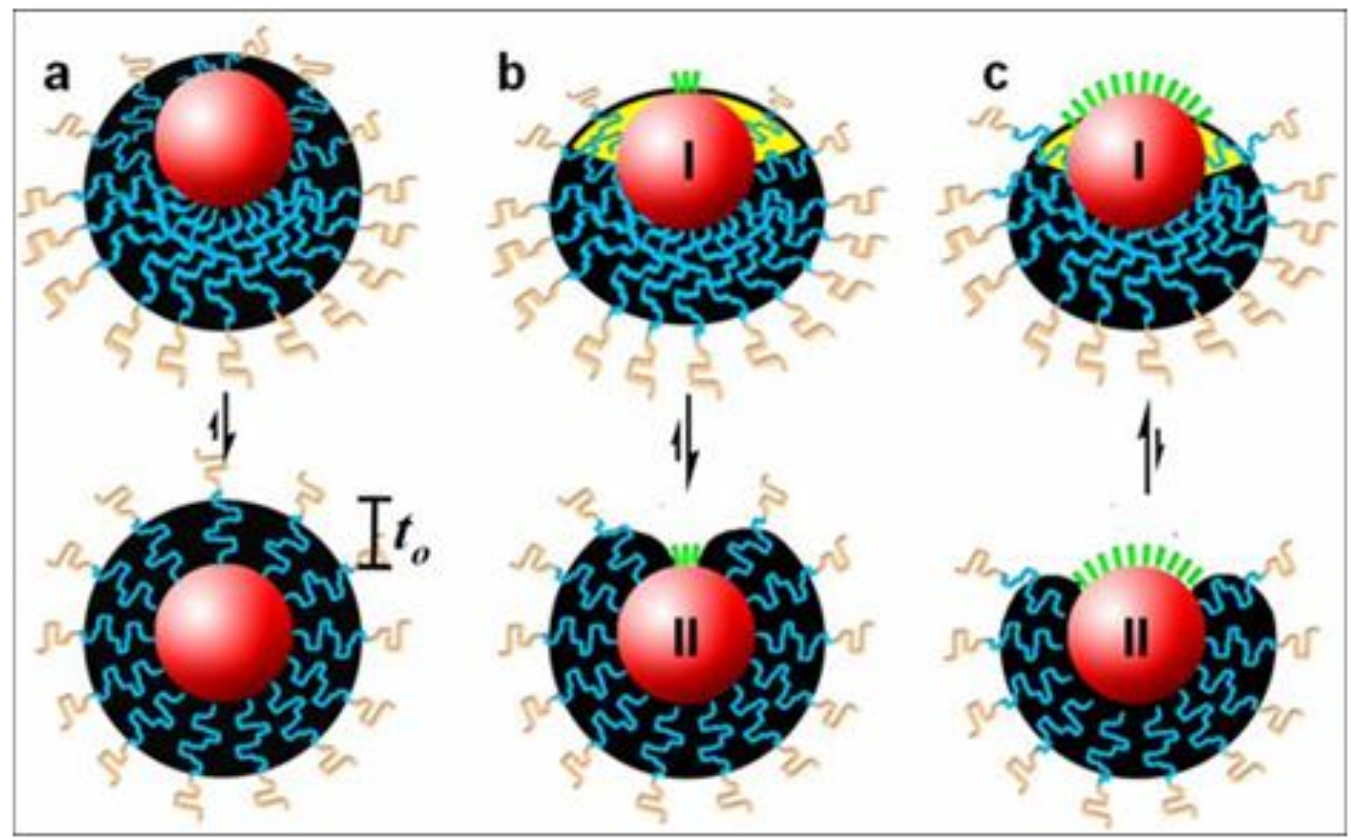

Figure 5.4. Scheme of preferred relative position of polymer and AuNP when a)

no opening, b) small opening and c) larger opening.

localized energy penalties as one side of the polymer would be thinner and the other side thicker than $t_{0}$ (Figure. 5.4a). When mixed ligands were used, however, close examination of the PSPAA shell on $\left(\mathrm{L}_{\mathrm{A}}+\mathrm{L}_{\mathrm{B}}-\mathrm{AuNP}\right)$ had both thinner and thicker regions than $t_{0}$. Using the controllable anisotropic attachment polymer micelles on $\left(\mathrm{L}_{\mathrm{A}}+\mathrm{L}_{\mathrm{B}}-\mathrm{AuNP}\right)$, we could further investigate the forces acting on PSPAA for this anisotropic system. The position of the AuNP is trapped anisotropically in the micelle due to the presence of $\mathrm{L}_{\mathrm{B}}$ in which the interfacial tension between the PS and $L_{B}$ is high and unfavourable for attachment of PSPAA.

Scrutiny of the PSPAA polymer shell shape when the extent of anisotropicity was varied revealed a surprising transition from Figure. 5.2c and 5.2d. The two 


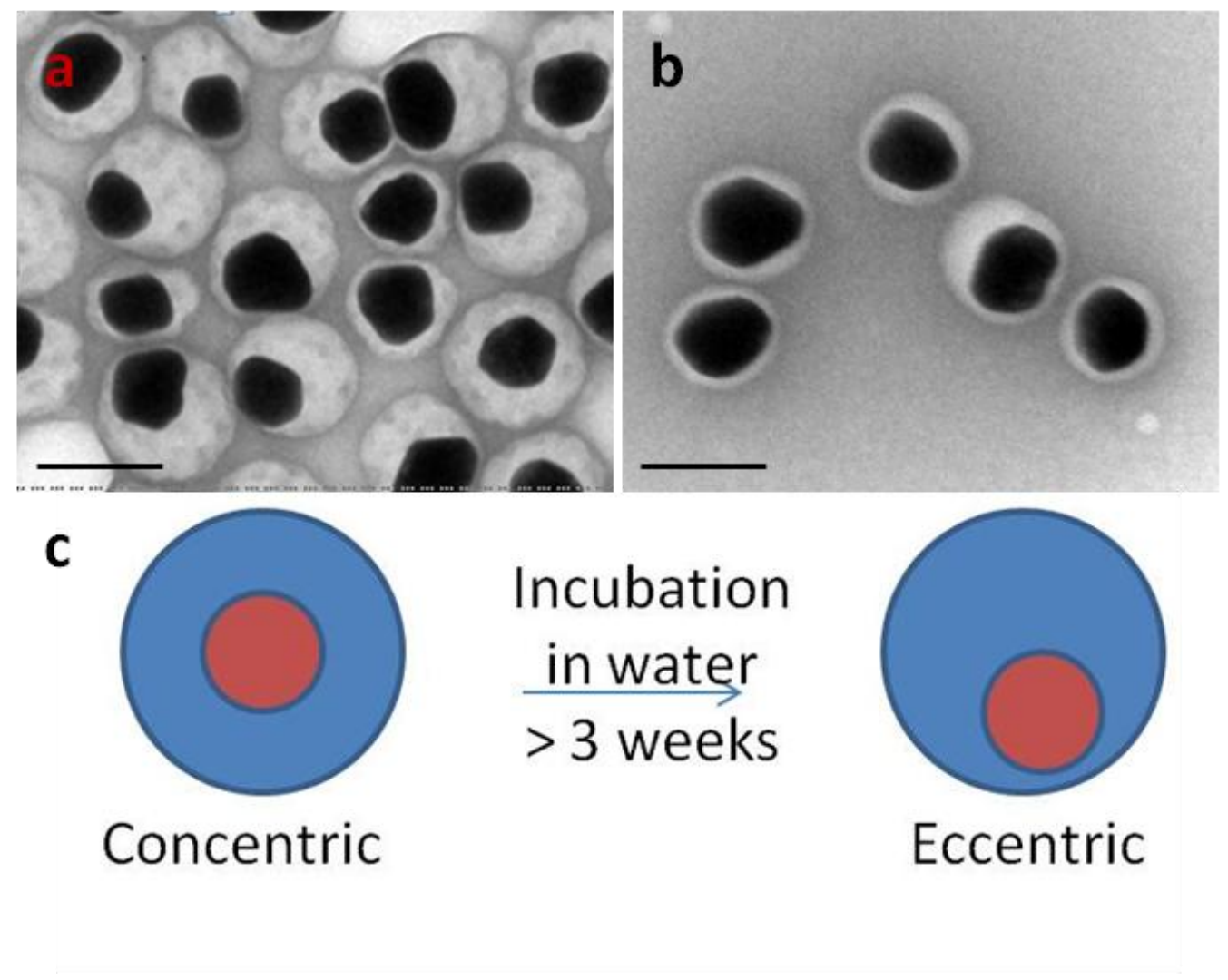

Figure 5.5. TEM images showing purified AuNP@PSPAA (a) before incubation;

(b) incubated in water for more than three weeks; (c) Core-shell NP turned eccentric.

polymer shell morphologies observed and redrawn schematically in Figure. 5.4 are indicative of distinct balances in the internal forces. The polymer morphologies of type I in Figure 5.4, corresponding to the structure for $\left(\mathrm{L}_{\mathrm{A}}+\mathrm{L}_{\mathrm{B}}-\mathrm{AuNP}\right) @$ PSPAA for Figure 5.2d, maintains an overall spheriodal shape leaving a thick lobe around the AuNP. On the other hand, type II polymer in Figure 5.4, characteristic of $\left(\mathrm{L}_{\mathrm{A}}+\mathrm{L}_{\mathrm{B}}-\mathrm{AuNP}\right) @$ PSPAA for Figure 5.1c, have uniform shell thickness on the $\mathrm{L}_{\mathrm{A}}$ coated areas of the AuNP. It was noted that when the opening is small, the polymer morphology type II is preferred, while a 
larger opening favored type I. A possible explanation for this phenomenon is by considering the internal forces of the structure. The very thin shell on AuNP surface in type I (highlighted) micelles are highly unstable due to the localized high surface/volume ratio, weak charge repulsion, and entropic penalty as the polymer has to be restricted or stretch in a thin layer. Despite the unfavorable forces at the thin front, the forces are countered by the low overall surface/volume ratio. Using $\mathrm{PS}_{404} \mathrm{PAA}_{62}$ which has a longer PS chain for the anisotropic encapsulation, the polymer morphology was found to be most often type I. This result supports the proposed forces of action as the longer PS chain permits more room for the polymer to be stretched or fold with lesser entropic penalty and conform to a state that lowest overall surface/volume ratio. For larger $\mathrm{L}_{\mathrm{B}^{-}}$covered area (Figure 5.4c), type I would be more favourable as the incurred thin-layer penalty is less significant (smaller highlighted area). Smaller $\mathrm{L}_{\mathrm{B}}$-covered area (Figure 5.4b) would involve a higher thin layer penalty (larger highlighted area) if it maintains type I morphology. For the smaller $\mathrm{L}_{\mathrm{B}}$-covered area, type II morphology was observed. This structure is less stable in terms of having higher surface/volume ratio as the shell is not perfectly spherical but this structure has low entropic penalty in stretching or PS chain to be shorter or longer than its unperturbed length. Figure 5.5 can show the TEM images of Au@PSPAA core-shell structures under different conditions there, which is consistent with the scheme in Figure5.4. 


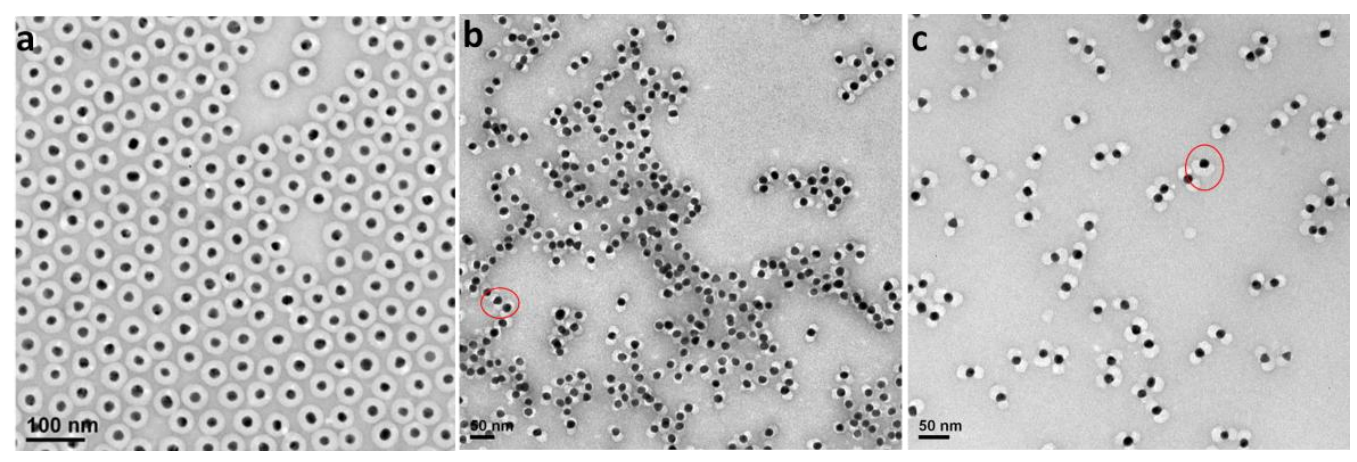

Figure 5.6. TEM images of the exposed AuNPs and empty PSPAA micelles obtained under the conditions with different $\mathrm{HCl}$ concentration: (a) 0, (b) $1 \mathrm{mM}$, (c) $10 \mathrm{mM}$ respectively. It is clear to see that the PSPAA shell formation from full encapsulation to partial encapsulation while $\mathrm{HCl}$ present there.

Besides, when introduce $\mathrm{HCl}$ into the PSPAA coating process we can control the Au NP@PSPAA core-shell morphology form core-shell to Janus, dumbbell, and no coating nanostructures. This is similar like the process in figure 5.6, where we can check the shell of PSPAA is from full encapsulation to partialencapsulation. Here it is possible that $\mathrm{Cl}^{-}$ions can change the Au NPs surface from positive charges to neutral and then will decrease the interfacial energy between Au NPs and PSPAA shell. So According to ions concentration control we can control the PSPAA shell encapsulation process from full encapsulation to partial encapsulation as shown in figure 5.6.

\subsubsection{Solvent ratio effect}

It is clear and interesting to see that different morphology of Au NP@PSPAA nanostructures can be collected while we tune the solvent ratio of DMF/water for 
the encapsulation process.

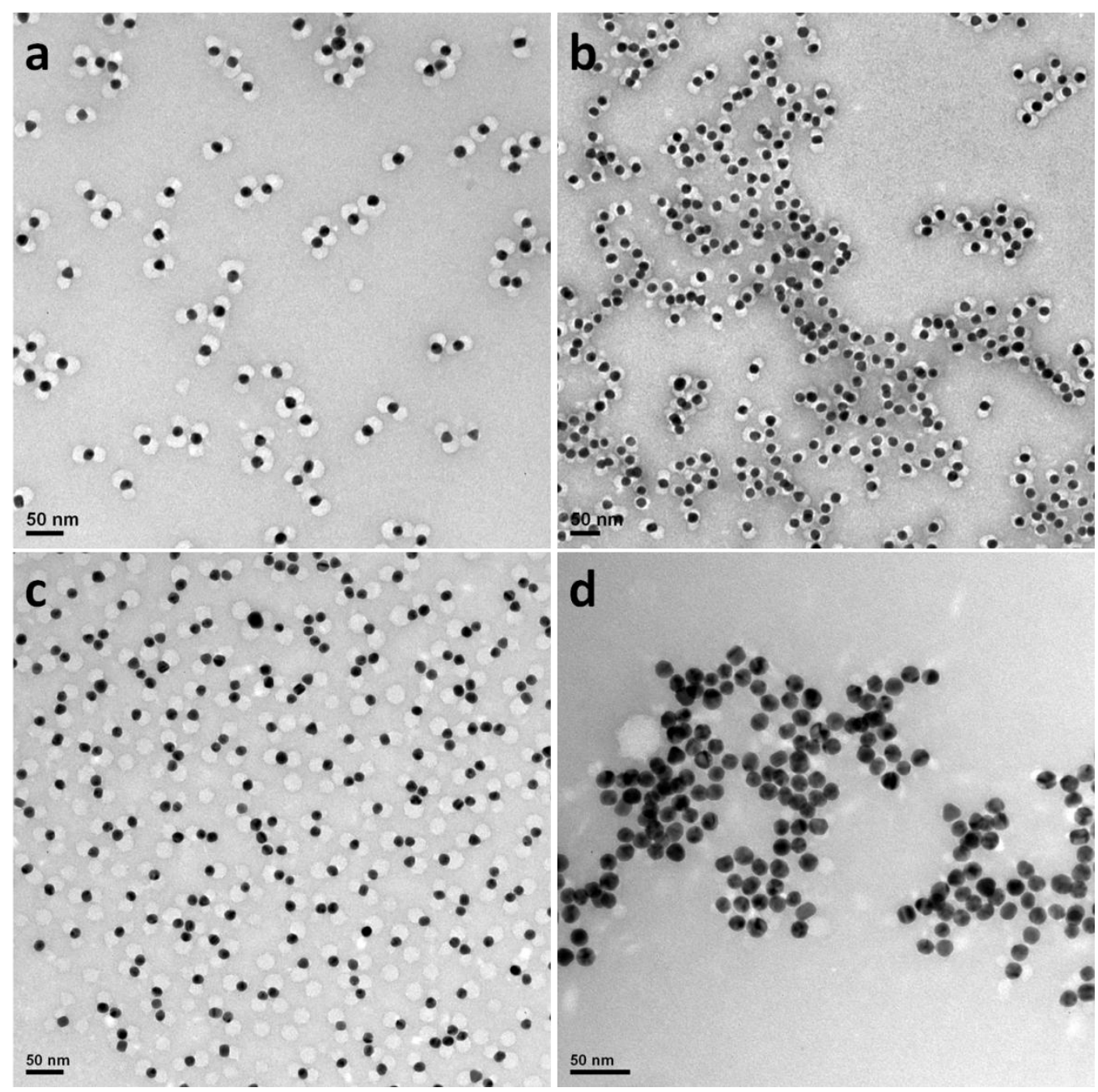

Figure 5.7. TEM images of dumbbell-like AuNP@PSPAA with spherical ends

obtained when solvent ratio (DMF/water) was tuned (a) $10 / 1$; (b) $7.8 / 1$; (c) $4.5 / 1$;

(d) $2.7 / 1$. It is clear to see the PSPAA shell morphology is changed with the

solvent ratio difference.

It is clear that the solvent ratio can affect the polymer micelle assembly and the nanoparticle aggregation during the encapsulation. More water content can lead to partial encapsulation during the preparation procedure, and the reason is because with the water content decreases, the solution became of lower 


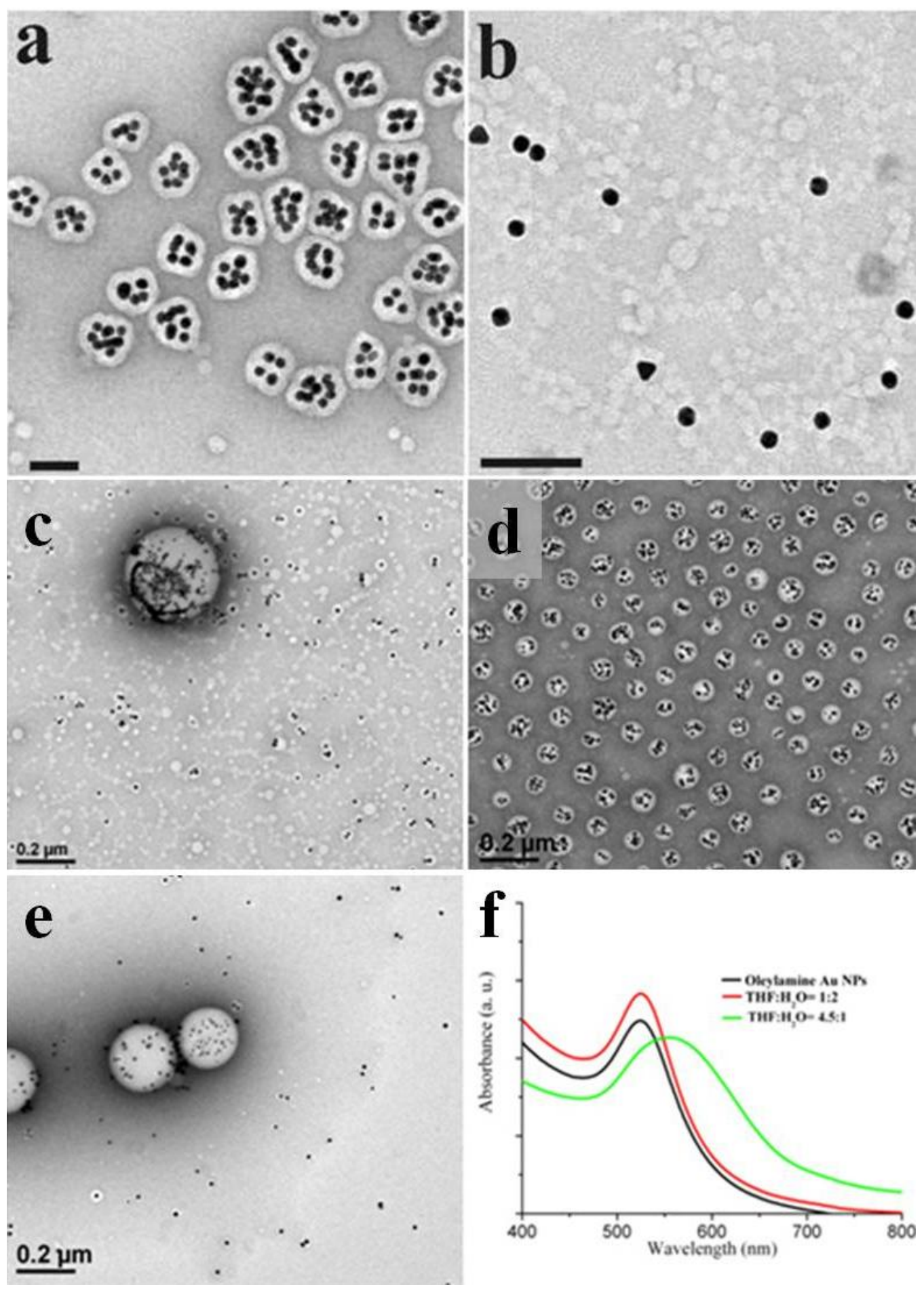

Figure 5.8. TEM images of citrate-Au NPs encapsulated by $\mathrm{PS}_{154}-b-\mathrm{PAA}_{60}$,

which prepared when (a) $\mathrm{DMF} / \mathrm{H}_{2} \mathrm{O}=8 / 1$, (b) $\mathrm{DMF} / \mathrm{H}_{2} \mathrm{O}=1 / 1$, and TEM images of encapsulated oleylamine-Au NPs prepared in (c) $\mathrm{THF} / \mathrm{H}_{2} \mathrm{O}=8 / 1$, (d) $\mathrm{THF} / \mathrm{H}_{2} \mathrm{O}=4.5 / 1$, (e) $\mathrm{THF} / \mathrm{H}_{2} \mathrm{O}=1 / 2$. (f) $\mathrm{Uv}$-vis spectra of oleylamine-Au NPs 
before (black), after encapsulation in $\mathrm{THF} / \mathrm{H}_{2} \mathrm{O}=4.5 / 1$ (green) and $\mathrm{THF} / \mathrm{H}_{2} \mathrm{O}=$ $1 / 2$ (red). Scale bars are $100 \mathrm{~nm}$.

dielectric constant. Consequently, the electrostatic repulsion will be decreased among NPs leading to the nanoparticle aggregation at lower water content. To the solvent ratio effect, especially the water content on the coating morphology. Regarding a bad solvent for the hydrophobic block of the copolymer, water works as precipitant while mixing with the solution of block copolymers in the good solvent. Previous report showed that addition of water was necessary to induce self-assembly of block copolymer. In our system, the volume ratios of common solvent to $\mathrm{H}_{2} \mathrm{O}$ determined the aggregation level of NPs, as well as the morphology of NP-block copolymer assemblies.

In the case of hydrophilic AuNPs, aggregation was obtained with 8/1 of $\mathrm{DMF} / \mathrm{H}_{2} \mathrm{O}$ (Figure 5.8a), while poor encapsulation was occurred when $1 / 1$ of $\mathrm{DMF} / \mathrm{H}_{2} \mathrm{O}$. (Figure 5.8b). According to the Derjaguin Landau Verwey and Overbeek theory (DLVO theory), the stability of NPs in solution is controlled by two factors, namely electrostatic repulsion $\left(\mathrm{V}_{\text {elec }}\right)$ and Van de Waals attraction $\left(\mathrm{V}_{\mathrm{vdw}}\right)$. Weakening $\mathrm{V}_{\text {elec }}$ between NPs usually causes aggregation, and a decrease in the dielectric constant of the medium surrounding NPs can cause a decrease of $\mathrm{V}_{\text {elec. }}$ In our system, it has been known that the dielectric constant of water $(\varepsilon=80)$ is higher than that of DMF $(\varepsilon=38.2)$ and THF $(\varepsilon=7.5)$. As the water content decreases, dielectric constant of the solution becomes lower. Consequently, the 
electrostatic repulsion among NPs decreases, thus aggregation of NPs at lower water content is observed. On the other hand, when the water content increased within a certain range, the high dielectric constant may contribute to the non-aggregation of NPs in solution. However, if water content continues increasing, the solvent obtains poorer quality for hydrophobic PS block. As a response, the stretching degree of PS chain decreases, resulting in a negative influence on the interaction between PS chain and surface hydrophobic ligand. PSPAA assemblies surrounding NPs are limited and partial encapsulation was observed.

Compared to hydrophilic NPs, nanostructure of hydrophobic NP-block copolymer assemblies were different. When the ratio of $\mathrm{THF} / \mathrm{H}_{2} \mathrm{O}$ increased to 8/1, as shown in Figure 5.8c, some large spherical micelles containing a large amount of hydrophobic AuNPs were obtained due to the decreasing dielectric constant of mixed solvent. However, when the ratio of $\mathrm{THF} / \mathrm{H}_{2} \mathrm{O}$ decreased to $1 / 2$, some partial encapsulated structure as well as large micelles contained mutli-core could be observed (Figure 5.8d-f), as hydrophobic NPs are not tended to aggregated in higher water content mixture. 


\subsubsection{Opening end control}

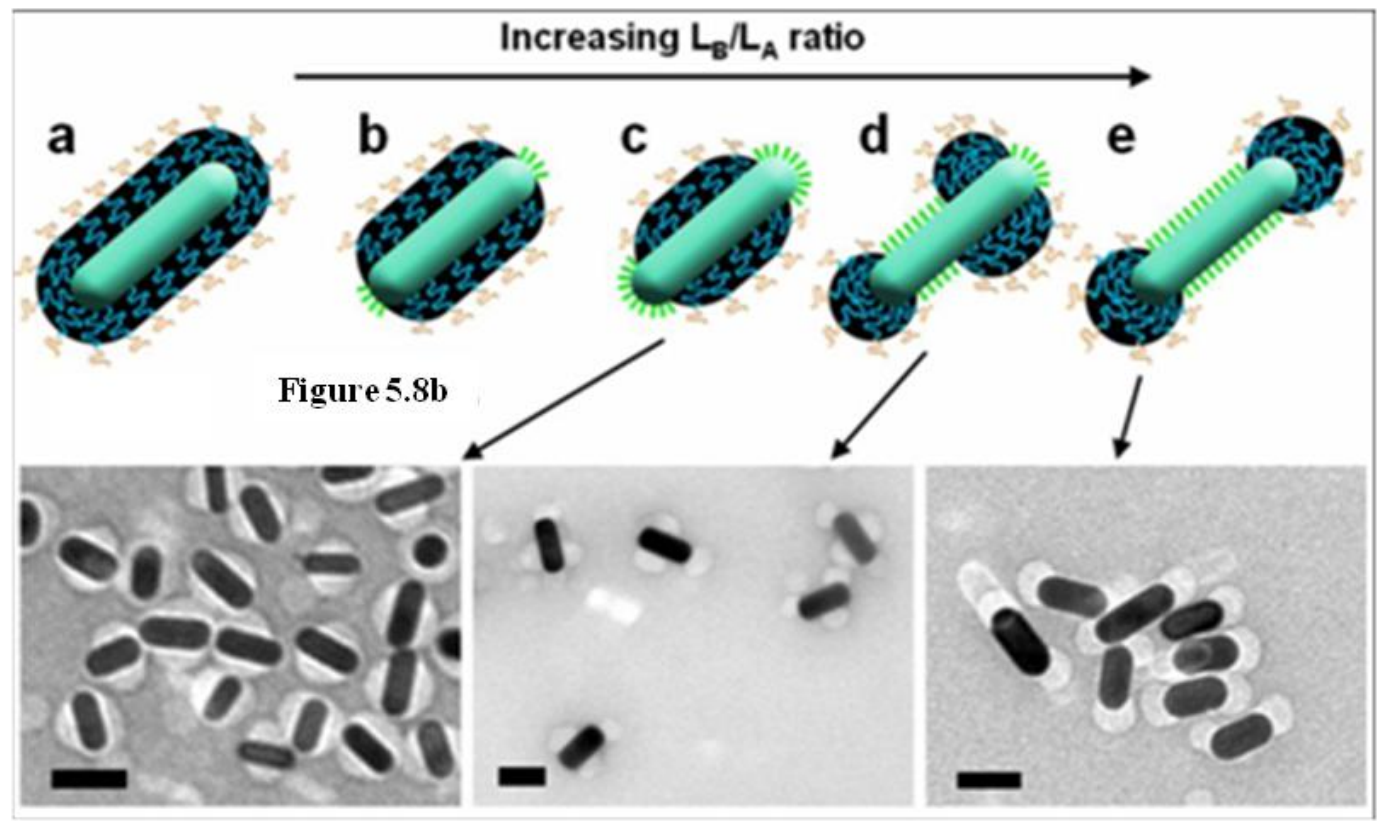

Figure 5.9. Scheme of AuNR@PSPAA structures with increasing $L_{B} / L_{A}$ ratio and the corresponding TEM images. Scale bars: $50 \mathrm{~nm}$.

Ultimately by understanding the structure of the mixed ligand shell and the nature of assembly of PSPAA position, we hope to be able to predict where the openings on the polymer shell would form. In our previous paper, the openings in the polymer shells most often occur at high-curvature positions on the AuNP chain ruling out the effect of facet preference for the binding of $L_{B}$ or specific packing patterns for $\mathrm{L}_{\mathrm{A}} \cdot{ }^{7}$ Instead, the strain of polymer micelles at high-curvature points seems to be the dominant factor in determining polymer distribution. Based on the previous observation, we further use an anisotropic metal, AuNRs to investigate the selective positioning of the opening.

Interestingly, increasing $\mathrm{L}_{\mathrm{B}} / \mathrm{L}_{\mathrm{A}}$ ratio resulted in the transition of several 
polymer morphologies on AuNRs with the transition from Figure. 5.8c to e being most intriguing. The extent of $\mathrm{L}_{\mathrm{A}}$-covered surface which continuously decreases at increasing $\mathrm{L}_{\mathrm{B}} / \mathrm{L}_{\mathrm{A}}$ ratio remains consistent with the AuNP systems (Figure. 5.9). At low $\mathrm{L}_{\mathrm{B}}$ concentrations, the polymer demonstrates preferential formation of openings at high curvature regions as observed previously. However as the $L_{B}$ concentration increases, the strain from overall polymer attachment become less a factor as patched attachment of micelles dominates (Figure. 5.9d) and finally, PSPAA attaches at the ends of AuNRs to achieve minimal repulsion between PAA chains and PS-water surface tension (Figure 5.9e). This observation further substantiates the argument against facet preference of the attached ligand shell but also suggests that the competition of $\mathrm{L}_{\mathrm{A}} / \mathrm{L}_{\mathrm{B}}$ in the presence of polymer is a dynamic process which leads to the minimization of total free energy in the system. If the exchange is not dynamic we would not have observed the shift of polymer from the sides to the ends of the NRs.

\subsubsection{PSPAA shell mobility control}

When we coat Te NWs by PSPAA shell in the similar system, an interesting phenomenon was checked there: with the temperature and solvent ratio difference it gives different kinds of PSPAA shells.

Te nanowires were synthesized by $\mathrm{TeO}_{2}$ reduced reaction by hydrazine hydrate under the room temperature, and then the nanowires were stabilized by PVP 40 $\mathrm{mM}$ aqueous solution before used as the seed for encapsulation. 


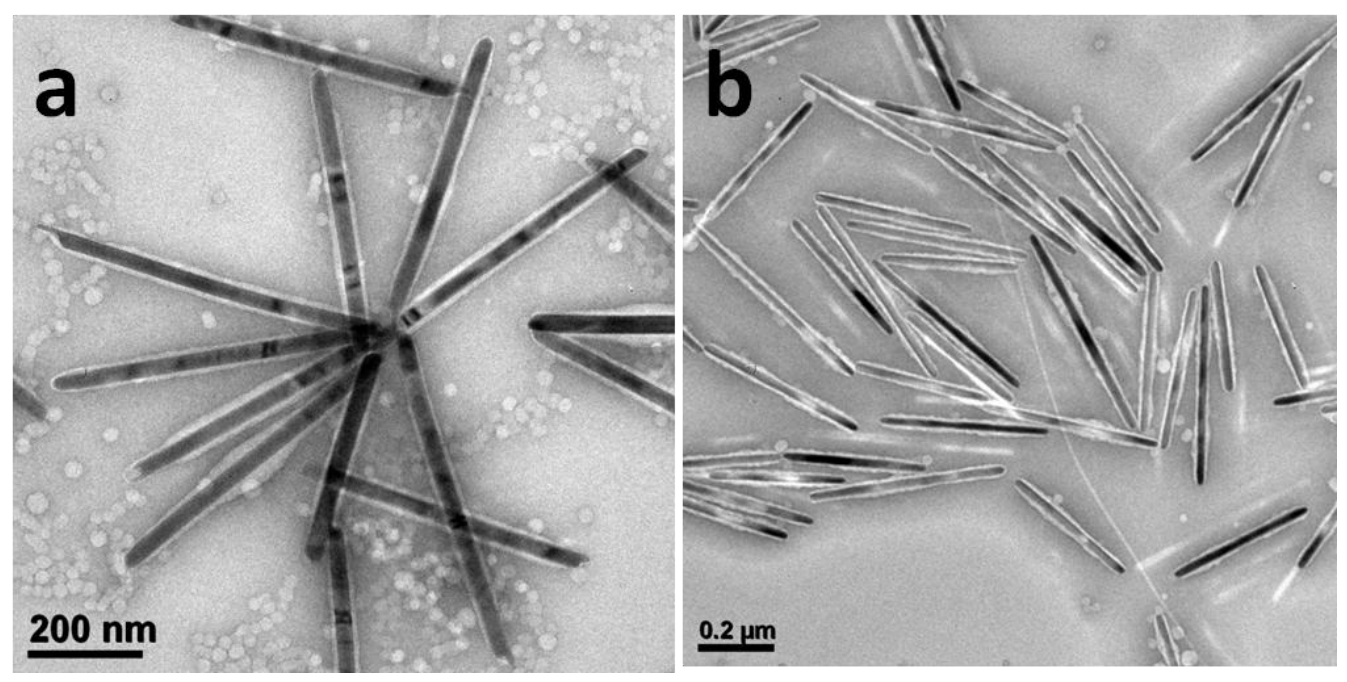

Figure 5.10. TEM images of (a) $50 \mathrm{~nm}$ Te@PSPAA, and (b) $20 \mathrm{~nm}$ Te@PSPAA under the solvent ratio of DMF/water $4.5 / 1$, at $110{ }^{\circ} \mathrm{C}, 2$ hours.

Te NW was centrifuged and then purified 3 times to remove the over excess PVP inside the solution before used as full encapsulation. Then the Te NW was dispersed in $\mathrm{DMF} /$ water solution under different solvent ratio, followed by PSPAA aqueous solution addition $(8 \mathrm{mg} / \mathrm{mL})$, then the mixture was heated at 110 ${ }^{\circ} \mathrm{C}, 2$ hours. According to the encapsulation process, we control the Te NW concentration and PSPAA concentration similar at all case to compare. Besides, we also tune the order of different material addition and found that there is no great difference there, which means that the chemical addition order, the concentration, as well as the reaction time is not the key factor to encapsulation control.

Firstly, we tune the Te NW diameter to check the shell morphology, and it is clear to see from figure 5.10, which can show both Te NW sample give core-shell nanostructures there. So it is rule out the Te NW diameter can affect the shell 
deposition process during the PSPAA encapsulation.

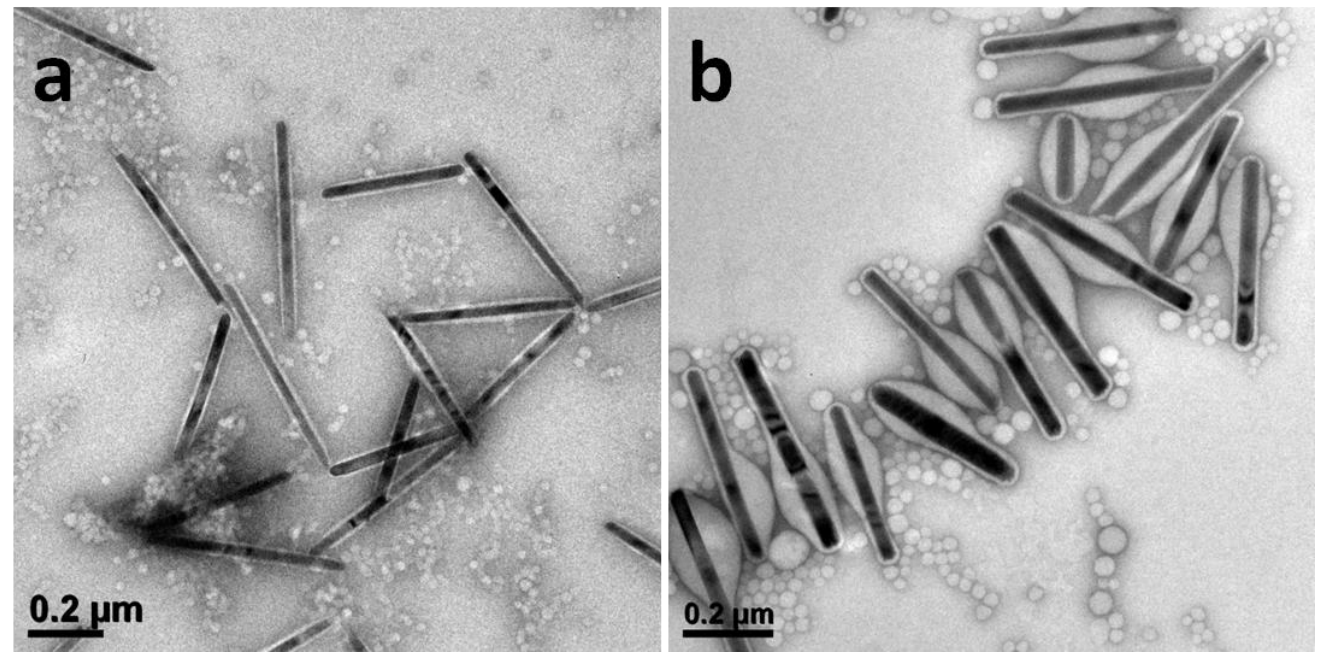

Figure 5.11. TEM images of Te@PSPAA synthesized under different solvent ratio (a) $\mathrm{DMF} /$ water $=4.5 / 1$, and (b) $\mathrm{DMF} /$ water $=9 / 1$, at $110{ }^{\circ} \mathrm{C}, 2$ hours.

Secondly, we tune the solvent ratio during the Te NW encapsulation procedure.

The DMF/water ratio can affect the polymer swelling, the micelle formation, and the nanowire aggregation in the whole encapsulation process. So we hope to trap any PSPAA shell morphology change under different solvent ratios. From figure 5.11 we can find that the PSPAA shell thickness in the middle will be thicker than that of the end side while the solvent ratio was increased from $4.5: 1$ to $9: 1$. So it is possible that the higher solvent ratio can lead to higher mobility of the polymer shell, which will move towards to the Te NW middle and giving gourd like structure.

In order to test the polymer shell mobility effects on the shell morphology we do other control experiments: keep all other parameters the same while the heating temperature was different, which will affect the polymer deposition, the 
micelle formation and then in return changing the PSPAA shell mobility. The PSPAA shell mobility is a case of thermodynamic control, which means that different temperature changes the stability of the polymer shell, and then can lead to different level of the PSPAA shell mobility during the encapsulation.

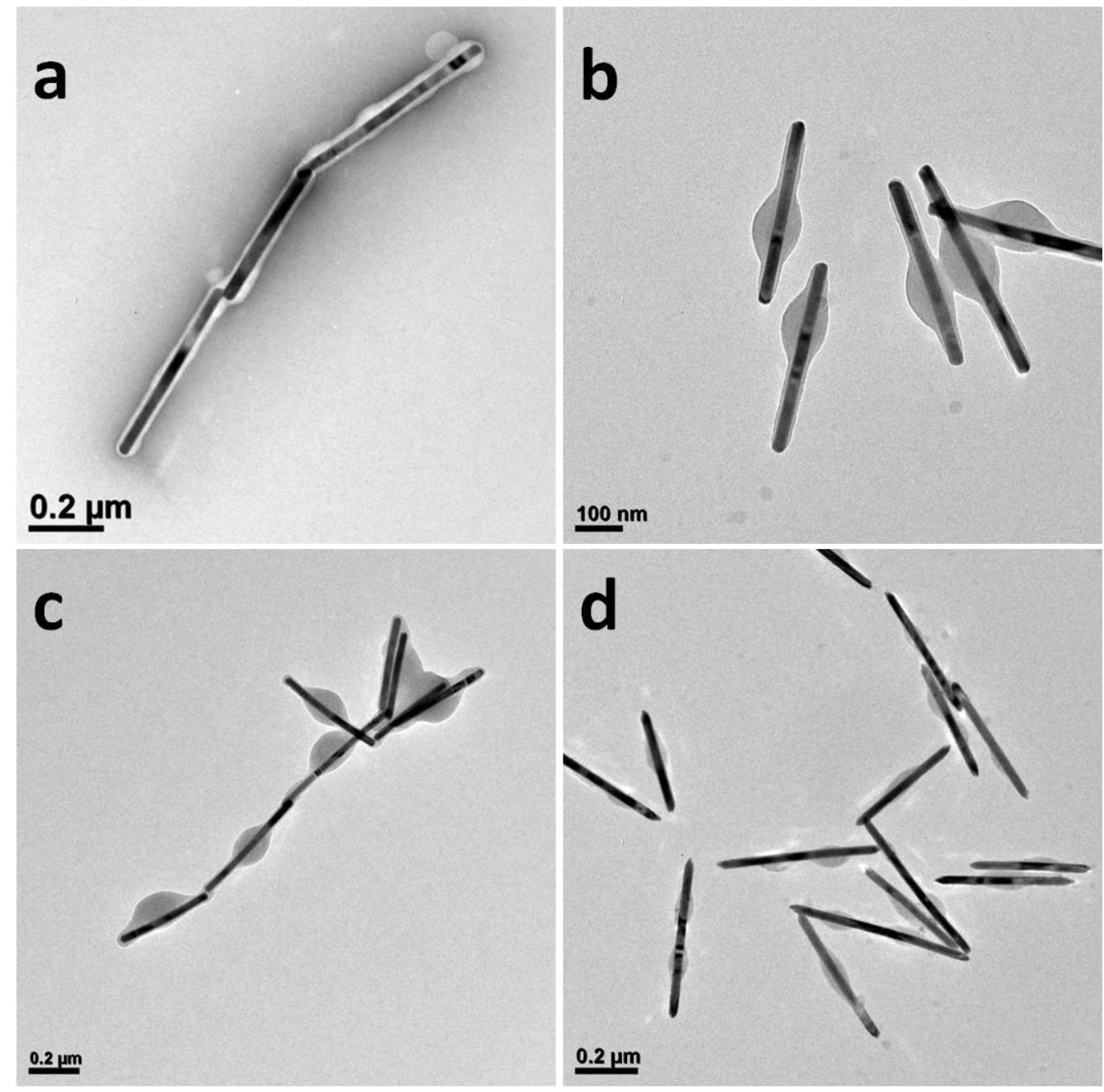

Figure 5.12. TEM images of Te@PSPAA synthesized under different solvent ratio $\mathrm{DMF} /$ water $=4.5 / 1$, at different temperature (a) $110{ }^{\circ} \mathrm{C}$, (b) $100{ }^{\circ} \mathrm{C}$, (c) $90{ }^{\circ} \mathrm{C}$, (d) $80{ }^{\circ} \mathrm{C}, 2$ hour encapsulation.

It is obviously that the temperature can also affect the polymer swelling process, and the swelling can also affect the final shell morphology there. According to 
figure 5.12 we can find that the polymer mobility is better at $90-100{ }^{\circ} \mathrm{C}$ which gives gourd like structures. The higher $\left(110^{\circ} \mathrm{C}\right.$ or higher $)$ or lower $\left(80{ }^{\circ} \mathrm{C}\right)$ is not good for polymer mobility. Here the temperature data support our hypothesis about polymer mobility control. We have tried the encapsulation at higher

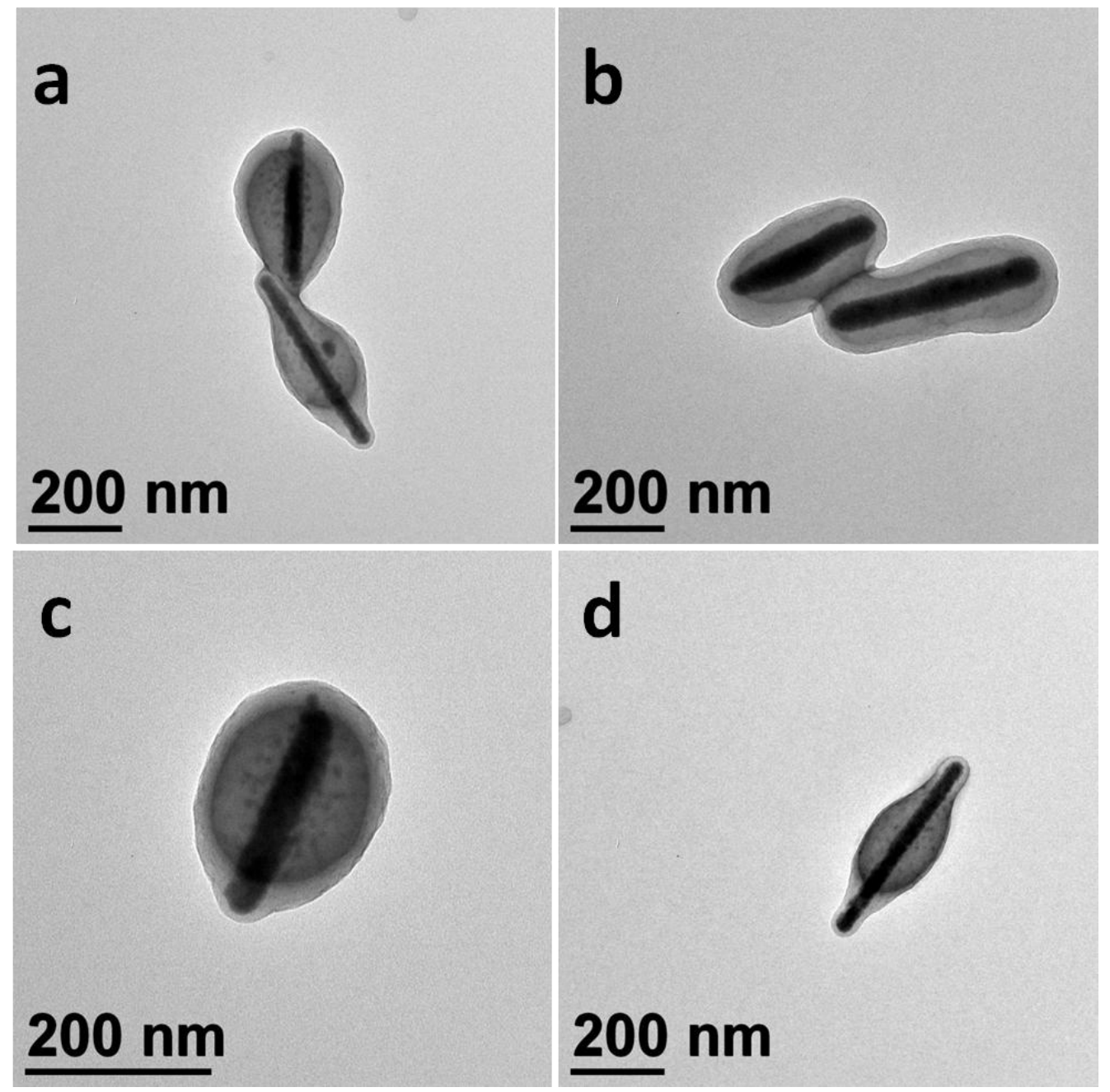

Figure 5.12. TEM images of Te@PSPAA synthesized under different solvent ratio $\mathrm{DMF} /$ water $=9 / 1$, at different temperature (a) $100{ }^{\circ} \mathrm{C}$, (b) $90{ }^{\circ} \mathrm{C}$, ratio $\mathrm{DMF} /$ water $=7.8 / 1$ (c) $100{ }^{\circ} \mathrm{C}$, (d) $90{ }^{\circ} \mathrm{C}$, heating 2 hours to encapsulate the $\mathrm{Te}$ NW.

(d) $80{ }^{\circ} \mathrm{C}, 2$ hour encapsulation temperature $\left(130{ }^{\circ} \mathrm{C}\right)$ and it is similar with figure 
5.11d, most of the Te NW cannot be coated by PSPAA shell. The possible reason is that the temperature scale is critical to the polymer shell formation.

In order to get novel structure we tried to combine the above condition: tuning the solvent ratio linked to tuning the temperature during the $\mathrm{Te} \mathrm{NW}$ encapsulation process, and hope to get a window for new control and future application in metal@PSPAA nanostructures. It is interested to get the new type of nanostructure as shown in figure 5.11, which show the double layer of PSPAA shell and gourd like structure.

\subsection{Conclusion}

We have investigated in detail the driving forces in forming the anisotopic structures by specifically identifying the ligand distribution on the AuNPs surface. The ligand shell was found to have significant phase segregation with $\mathrm{L}_{\mathrm{A}}$ attached to the polymer and $\mathrm{L}_{\mathrm{B}}$ exposed to the solution. The PSPAA shell preferentially attaches on the $\mathrm{L}_{\mathrm{A}}$ regions on the metal core and adapt to the morphology with the lowest overall surface energy. The phase segregation of the mixed ligands may have been promoted in the presence of PSPAA, dynamic exchange of the ligand and PSPAA was observed with anisotropic cores. This dynamic exchange strives to achieve the state with minimal surface energy depending on the ration of $\mathrm{L}_{\mathrm{A}} / \mathrm{L}_{\mathrm{B}}$. This opens up the opportunity to specifically position the patches of ligands by manipulating the attachment of PSPAA. This work has been largely qualitative based on TEM observation which bypasses 
other more quantitative characterization methods that work for large or flat surfaces. We hope it is helpful to bottom up fabrication in future 


\subsection{Bibliography}

(1). Z. Mao, H. Xu and D. Wang, Adv. Funct. Mater., 2010, 20, 1053-1051-1074.

(2). S. C. Glotzer and M. J. Solomon, Nat. Mater., 2007, 6, 557-562.

(3). T. M. Hermans, M. A. C. Broeren, N. Gomopoulos, P. v. d. Schoot, M. H. P. v.

Genderen, N. A. J. M. Sommerdijk, Nat. Nanotech., 2009, 4, 721-726.

(4). Z. Zhang and S. C. Glotzer, Nano Lett., 2004, 4, 1407-1413.

(5). A. B. Pawar and I. Kretzschmar, Macromol. Rapid Commun., 2010, 31, $150-168$.

(6). T. Chen, M. Yang, X. Wang, L. H. Tan and H. Chen, J. Am. Chem. Soc., 2008, $130,11858-11859$.

(7). L. H. Tan, S. Xing, T. Chen, G. Chen, X. Huang, H. Zhang and H. Chen, ACS Nano, 2009, 3, 3469-3474.

(8). M. Casavola, R. Buonsanti, G. Caputo and P. D. Cozzoli, Eur. J. Inorg. Chem., 2008, 837-854.

(9). P. D. Cozzoli, T. Pellegrino and L. Manna, Chem. Soc. Rev., 2006, 35, $1195-1208$.

(10). G. H. Ma, Z. G. Su, S. Omi, D. Sundberg and J. Stubbs, J. Colloid Interface Sci., 2003, 266, 282-294.

(11). S. H. Im, U. Jeong and Y. Xia, Nat. Mater., 2005, 4, 671-675.

(12). W. Yin and M. Z. Yates, Langmuir, 2008, 24, 701-708.

(13). S. Torza and S. G. Mason, J. Colloid Interface Sci., 1970, 33, 67-83. 
(14). S. Semal, C. Bauthier, M. Voué, J. J. V. Eynde, R. Gouttebaron and J. D. Coninck, J. Phys. Chem. B, 2000, 104, 6225-6232.

(15). C. Gentilini and L. Pasquato, J. Mater. Chem., 2010, 20, 1403-1412.

(16). L. Floreano, S. Scandolo, A. Morgante, M. L. Klein and G. Scoles, Science, 2008, 321, 943-946.

(17). G. E. Poirier, Chem. Rev., 1997, 97, 1117-1127.

(18). A. M. Jackson, Y. Hu, P. J. Silva and F. Stellacci, J. Am. Chem. Soc., 2006, $128,11135-1114911139$.

(19). A. M. Jacson, J. W. Myerson and F. Stellacci, Nat. Mater., 2004, 3, 330-336.

(20). A. Santos, C. Singh and S. C. Glotzer, Phys. Rev. E, 2010, 81, 011113.

(21). C. Singh, P. K. Ghorai, M. A. Horsch, A. M. Jackson, R. G. Larson, F.

Stellacci and S. C. Glotzer, Phys. Rev. Lett., 2007, 99, 226106.

(22). M. Lucarini and L. Pasquato, Nanoscale, 2010, 2, 668-676.

(23). J. C. Love, L. A. Estroff, J. K. Kriebel, R. G. Nuzzo and G. M. Whitesides, Chem. Rev., 2005, 105, 1103-1169.

(24). C. Vilain, F. Goettmann, A. Moores, P. Le Floch and C. Sanchez, Journal of Materials Chemistry, 2007, 17, 3509-3514.

(25). L. Zhang and A. Eisenberg, Science, 1995, 268, 1728-1731.

(26). H. Y. Chen, S. Abraham, J. Mendenhall, S. C. Delamarre, I. K. Kahli Smith and C. A. Batt, Chem. Phys. Chem., 2008, 9, 388-392.

(27). Y. Yu, L. Zhang and A. Eisenberg, Macromolecules, 1998, 31, 1144-1154. 


\section{Chapter 6 Homo- and Co-Polymerization of Polysytrene-Block-Poly(Acrylic Acid)-Coated Nanoparticles \\ Metal}

\subsection{Introduction}

Nanoparticles (NPs) are the basic building blocks in nanoscience. They can be viewed as "atoms" for constructing more complex superstructures. While such a vision has a long history, we are far from materializing it. The field of nano-assembly is still at its infancy, where synthetic advance is the main thrust for new properties and new applications.

In assembly, the orderliness by which the components are put together is of critical importance for achieving functionality. Three-dimensional (3D) assembly can be likened to the construction of an engine from nuts and bolts, whereas 1D assembly of NPs can be compared to the polymerization of organic monomers. In terms of achieving spatial precision for building sophisticated and functional structures, the former is significantly more challenging than the latter, but even the latter has yet to be fully realized.

In comparison to the 1D assembly of homologous NPs, the assembly of 


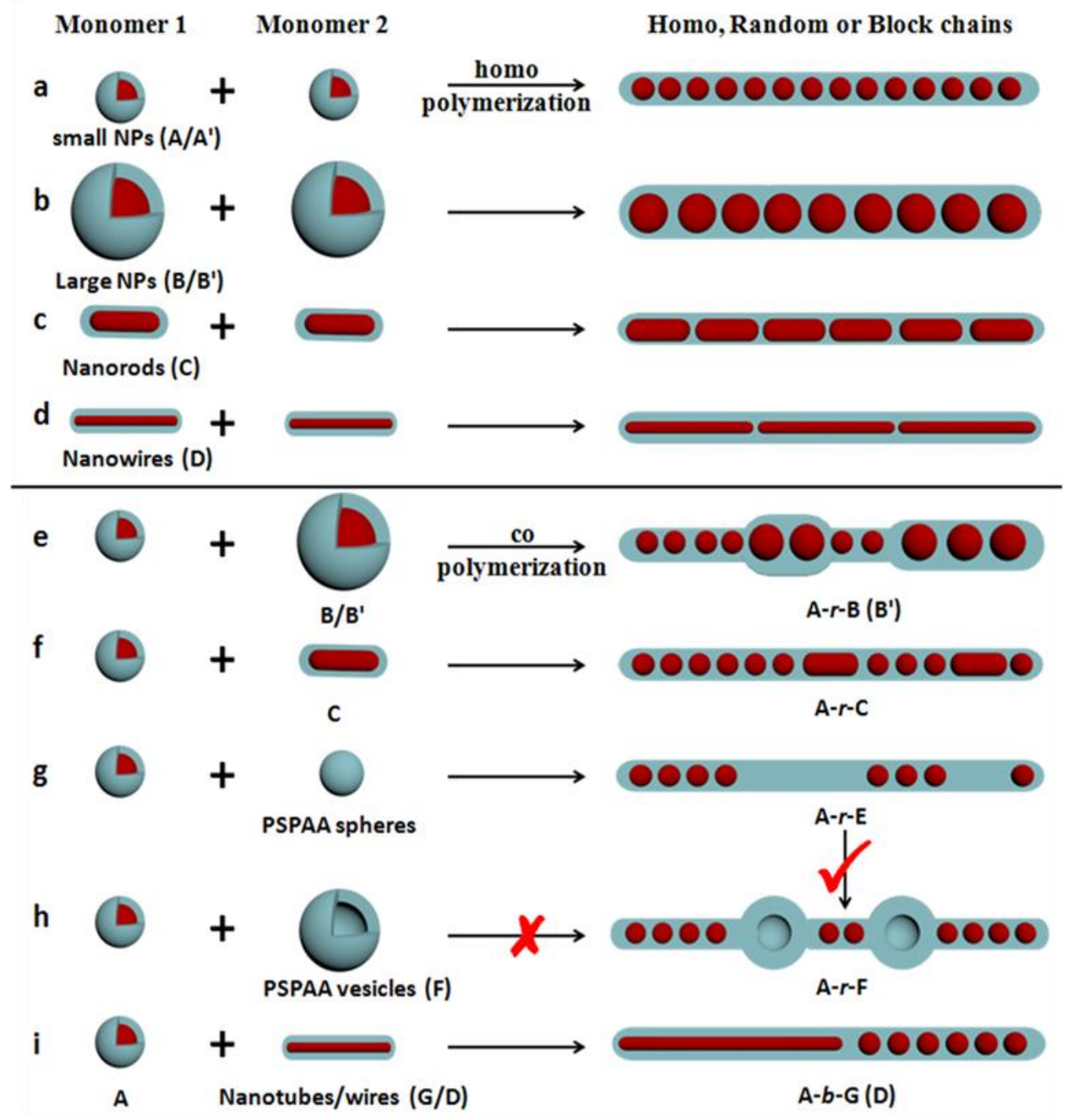

Figure 6.1. Schematics illustrating (a-d) the "polymerization" of core-shell NPs into "homopolymers"; (e-i) the "copolymerization" of core-shell NPs into random or block "copolymers". Reprinted with permission from ref. [59], copyright 2014 American Chemical Society.

heterogonous NPs provides a much richer structural variety, which can be explored for multifunctional and collective properties. Such hetero-assembly is in a way similar to the copolymerization of organic monomers, where an 
enormous variety of copolymers have been synthesized for many applications. Bio-polymers such as proteins and DNAs are the "crown jewels" of hetero-assembly, as they are essentially precisely controlled copolymers where the structural variety leads to functional dexterity. From this perspective, new capabilities in the hetero-assembly of NPs will offers novel means for exploring complex nanostructures and new properties.

In organic polymerization, the term "oligomer" typically refers to a polymer consisted of a few monomer units $(<20)$. In this context, most of the 1D assembly of NPs in the literature are more like oligomers 1 than polymers. ${ }^{2}$ The difficulty in obtaining long chains of NPs lies in the fact that colloidal NPs often have very similar "reactivity" (probability of successful collision) during their aggregation. As a result, their "polymerization" tends to follow the step-growth mode. ${ }^{1 b, 3}$ Basically, the monomers are quickly depleted and the later stage of assembly is dominated by the collision of large clusters, which is usually difficult and nonspecific. It would be thus impractical to give orderly long chains, unless the self-assembly process is guided by strong magnetic dipole. ${ }^{4}$

Recently, we reported an unconventional chain-growth "polymerization" mode in the assembly of core-shell NPs into ultralong chains. ${ }^{2 b}$ The shape of polystyrene-block-poly (acrylic acid) (PSPAA) micelles are known to transform from sphere to cylinder upon acid treatment. ${ }^{5}$ When AuNPs encapsulated in PSPAA shells (AuNP@PSPAA) were used as the monomers and subjected to 
acidic conditions, the PSPAA domains merged and transformed to cylindrical micelles, taking the embedded AuNPs along the way and "polymerizing" them into long chains (Figure 6.1). In this system, a few activated monomers were able to grow extensively into ultralong chains, whereas many monomers remained in the sample without experiencing any aggregation, indicating a chain-growth "polymerization" mode. ${ }^{2 b, 6}$ The presence of these monomers was essential in allowing the growth of the ultralong chains via sequential monomer addition.

Herein, we show that several other types of monomers can be integrated into the "polymerization" process, giving not only "homopolymers", but also random and block "copolymers" (Figure 6.1b-i). The viable monomers include AuNPs (d $=16$ and $32 \mathrm{~nm}$ ), Au nanorods (AuNRs), Te nanowires (TeNWs), and carbon nanotubes (CNTs), all of which are encapsulated in PSPAA shells before being used in the assembly. In addition, PSPAA cylinders and vesicles can also be inserted in between the above NPs in the chains. Our new method provides a facile means to construct hetero-assemblies of NPs in orderly chain configuration, where simple combination can lead to structural variety.

\subsection{Materials and methods}

\subsubsection{Materials}

All chemical reagents were used without further purification. Hydrogen tetrachloroaurate(III) hydrate, $99.9 \%$ (metal basis Au 49\%) was purchased from Alfa Aesar; 1,2-dipalmitoyl-sn-glycero-3-phosphothioethanol was purchased 
from Avanti Polar Lipids; amphiphilic diblock copolymer polystyrene-block-poly(acrylic acid) $\left(\mathrm{PS}_{154}\right.$-b-PAA $49, \mathrm{Mn}=16000$ for the PS block and $\mathrm{Mn}=3500$ for the PAA block, $\mathrm{Mw} / \mathrm{Mn}=1.15 ; \mathrm{PS}_{144}-\mathrm{b}-\mathrm{PAA}_{22}, \mathrm{Mn}=$ 15000 for the PS block and $\mathrm{Mn}=1600$ for the PAA block, Mw/Mn =1.11) were purchased from Polymer Source, Inc. Single-wall CNTs (carbonaceous purity 99\%) were purchased from NanoIntegris. Hydrochloric acid was purchased from P. P. Chemicals Sdn Bhd. Deionized water (resistance $>18.2 \mathrm{M} \Omega / \mathrm{cm}$ ) was used in all reactions. Copper specimen grids (300 mesh) with fervor/carbon support film were purchased from Beijing XXBR Technology Co.

The citrate-stabilized AuNPs (16, and $32 \mathrm{~nm}),{ }^{24}$ the CTAB-stabilized AuNRs ${ }^{25}$ and SDS-stabilized TeNWs ${ }^{18}$ were prepared by following the literature produces.

\subsubsection{Methods}

Characterization. TEM images were collected on a JEM-1400 (JEOL) transmission electron microscope operated at $100-120 \mathrm{kV}$. $\left(\mathrm{NH}_{4}\right)_{6} \mathrm{Mo}_{7} \mathrm{O}_{24}$ was used as stain $(3.4 \mathrm{mM})$ in all TEM images reported in this study.

General Synthesis of NP@PSPAA Monomers. The me-thod used here was modified from our previous report on the encapsulation of AuNPs.7a A solution of citrate-stabilized AuNPs $(\mathrm{d}=16 \mathrm{~nm}, 3 \mathrm{~mL})$ was concentrated to $20 \mu \mathrm{L}$ by centrifugation at $16000 \mathrm{~g}$ for $15 \mathrm{~min}$. The deep red solution collected at the bottom of the eppendorf tubes was diluted with $\mathrm{H}_{2} \mathrm{O}(162 \mu \mathrm{L})$. Then, the mixture was added to $818 \mu \mathrm{L}$ of PSPAA solution which was 
prepared by mixing $738 \mu \mathrm{L}$ DMF with PS $_{154}$-b-PAA 49 in DMF $(80 \mu \mathrm{L}, 8$ $\mathrm{mg} / \mathrm{mL}$ ). Finally, 1,2-dipalmitoyl-sn-glycero-3-phosphothioethanol in EtOH (40 $\mu \mathrm{L}, 2 \mathrm{mg} / \mathrm{mL}$ ) was added to the mixture. The final mixture has a volume of $1.04 \mathrm{~mL}$ with $\mathrm{VDMF} / \mathrm{VH}_{2} \mathrm{O}=4.5: 1$. It was then heated at $110{ }^{\circ} \mathrm{C}$ for $2 \mathrm{~h}$ and slowly cooled down till room temperature.

The same procedure was used to encapsulate AuNPs $(\mathrm{d}=32 \mathrm{~nm})$, and AuNRs with PSPAA. For the encapsulation of TeNWs, the procedure is similar to the that of AuNPs, except that ligand 2 was not added.

The single-wall CNTs was dispersed into $810 \mu \mathrm{L}$ of PSPAA solution which was prepared by mixing $730 \mu \mathrm{L}$ DMF with $\mathrm{PS}_{154}$-b-PAA 49 in DMF $(80 \mu \mathrm{L}, 8$ $\mathrm{mg} / \mathrm{mL}$ ), and then sonicated in an ice-water bath till to form transparent black solution. Finally, $\mathrm{H}_{2} \mathrm{O}(180 \mu \mathrm{L})$ was added drop wise to the above solution. The final mixture has a volume of $990 \mu \mathrm{L}$ with $\mathrm{VDMF} / \mathrm{VH}_{2} \mathrm{O}=4.5: 1$. It was then sonicated at $50{ }^{\circ} \mathrm{C}$ for $2 \mathrm{~h}$ and slowly cooled down till room temperature. $^{21}$

\section{Homo-assembly of NP@PSPAA, NR@PSPAA, and NW@PSPAA into}

chains. The "homo-polymerization" method used here was adapted from our previous report. ${ }^{2 \mathrm{~b}}$ The purified monomers AuNP@PSPAA $(\mathrm{d}=32 \mathrm{~nm}$, type B and B'), AuNR@PSPAA (type C), and TeNW@PSPAA (type D) were dispersed into a $\mathrm{DMF} / \mathrm{H}_{2} \mathrm{O}$ solution $\left(1 \mathrm{~mL}, \mathrm{VDMF} / \mathrm{VH}_{2} \mathrm{O}=6: 1\right) . \mathrm{HCl}(5 \mu \mathrm{L}$, $1 \mathrm{M})$ was then added into the solution, and the final concentration is $[\mathrm{HCl}]_{\text {final }}$ 
$=5 \mathrm{mM}$. The mixture was incubated at $60^{\circ} \mathrm{C}$ for $2 \mathrm{~h}$ to facilitate the shape transformation of PSPAA polymer shell.

Co-assembly of NP@PSPAA into Random Chains. The as-synthesized monomers were diluted ( $800 \mu \mathrm{L}$ diluted by $11.2 \mathrm{~mL}$ water), and then centrifuged at $16000 \mathrm{~g}$ for $30 \mathrm{~min}$ to remove the supernatant. The collected solution at the bottom of eppendorf tubes was diluted with $\mathrm{NaOH}(0.1 \mathrm{mM})$ and purified again. The final concentrated solution $(\sim 20 \mu \mathrm{L})$ contained the desired NP@PSPAA monomers free of DMF and empty PSPAA micelles. The concentrated AuNP@PSPAA (monomer A, d = $16 \mathrm{~nm}$ ) and monomer B (AuNP@PSPAA, $d=32 \mathrm{~nm}$ ) were dispersed into a $\mathrm{DMF} / \mathrm{H}_{2} \mathrm{O}$ solution $(1 \mathrm{~mL}$, $\left.\mathrm{VDMF} / \mathrm{VH}_{2} \mathrm{O}=6: 1\right) . \mathrm{HCl}(5 \mu \mathrm{L}, 1 \mathrm{M})$ was then added into the solution, and the final concentration is $[\mathrm{HCl}]$ final $=5 \mathrm{mM}$. The mixture was incubated at $60{ }^{\circ} \mathrm{C}$ for $2 \mathrm{~h}$ to facilitate the shape transformation of PSPAA polymer shell. The same procedure was used when monomer B' and C were employed in the place of B.

The random chains consisted of monomer A and PSPAA vesicles were prepared using a different method. Spherical micelles of PSPAA were used in place of PSPAA vesicles. First, $\mathrm{PS}_{154}$-b-PAA $49(80 \mu \mathrm{L}, 8 \mathrm{mg} / \mathrm{mL})$ in DMF was added $738 \mu \mathrm{L}$ DMF, and then water was added $(182 \mu \mathrm{L})$, making a solution of $\mathrm{VDMF} / \mathrm{VH}_{2} \mathrm{O}=4.5: 1$. The polymer solution was incubated at 110 ${ }^{\circ} \mathrm{C}$ for $2 \mathrm{~h}$ and slowly cooled down till room temperature. Second, the 
spherical PSPAA micelles $(62 \mu \mathrm{L})$ and purified monomer A were simultaneously dispersed into a $\mathrm{DMF} / \mathrm{H}_{2} \mathrm{O}$ solution $(938 \mu \mathrm{L}) . \mathrm{HCl}(5 \mu \mathrm{L}, 1 \mathrm{M})$ was then added into the solution, and the final concentration is $[\mathrm{HCl}]_{\text {final }}=5$ $\mathrm{mM}$ and the final $\mathrm{VDMF} / \mathrm{VH}_{2} \mathrm{O}=6: 1$. The mixture was incubated at $60{ }^{\circ} \mathrm{C}$ for a given period of time $(t=1.5-6 \mathrm{~h})$ to facilitate the shape transformation of PSPAA polymer micelles.

Co-assembly of NP@PSPAA into Block Chains. The purified CNT@PSPAA or TeNW@PSPAA was dispersed into a DMF/ $\mathrm{H}_{2} \mathrm{O}$ solution (1 $\left.\mathrm{mL}, \mathrm{VDMF} / \mathrm{VH}_{2} \mathrm{O}=6: 1\right)$ containing $2 \mu \mathrm{L}$ of $\mathrm{HCl}(1 \mathrm{M})$. The solution was heated at $60{ }^{\circ} \mathrm{C}$ for $20 \mathrm{~min}$, and then the monomer A (AuNP@PSPAA, d = 16 $\mathrm{nm})$ and $\mathrm{HCl}(1 \mathrm{M}, 3 \mu \mathrm{L})$ were added. The mixture was incubated at $60{ }^{\circ} \mathrm{C}$ for $2 \mathrm{~h}$ to facilitate the chain growth.

\subsection{Results and discussion}

To understand the mechanism in great detail will definitely help to control the system better. We suppose, combining with previous studies, that there are some factors that have influences on the formation of AuNP chains: the ratio of the solvent, temperature, ligands, polymer and acid. Under high ratio of $\mathrm{DMF} / \mathrm{H}_{2} \mathrm{O}$, it is believe that the micelle could dissociate or partially dissociated from the AuNPs surface. This dissociation is a result of the repulsion among PAA becoming weak, thus leading to the micelle difficult to maintaining their spherical structures. Further, in the presence of acid, the morphologies of the 
micelle should prone to be rod-like, which thus serve as template for the AuNP aggregation and as a protecting layer in solvent. Such a template formation of linear chains resemble to the seed-mediated growth of Au nanorods. The existing of the monomer may be the critical factor for self-promotion of long chain. Moreover, the swelling abilities of different ligands are different, it would affect the osmotic pressure between the gap as two micelles approach one another, which reduces the energy barrier for the adhesive collisions of micelles and enhances the efficient adhesive collisions among micelles. Therefore, the different polymers used and solvent ratios and the concentration of monomers should be investigated at the same time. The future work need to concentrate on studying the detail of this mechanism.

The monomers were prepared by PSPAA encapsulation as previously reported. $^{7}$ Taking the AuNP@PSPAA as an example, citrate-stabilized AuNPs were first functionalized with a layer of hydrophobic ligand $(\mathbf{1}, \mathbf{2}$, or $\mathbf{3}$, Figure 6.2a), upon which the amphiphilic PSPAA self-assembled into a uniform shell. The encapsulation was carried out under high DMF content and elevated temperature, which conditions were selected to promote the mobility of the PSPAA molecules. ${ }^{5 \mathrm{c}, 8}$ The hydrophobic PS blocks of the PSPAA have affinity to the hydrophobically functionalized NPs, whereas the hydrophilic PAA blocks dissolve in the solvent facing outwards. ${ }^{7}$ The resulting core-shell NPs were 


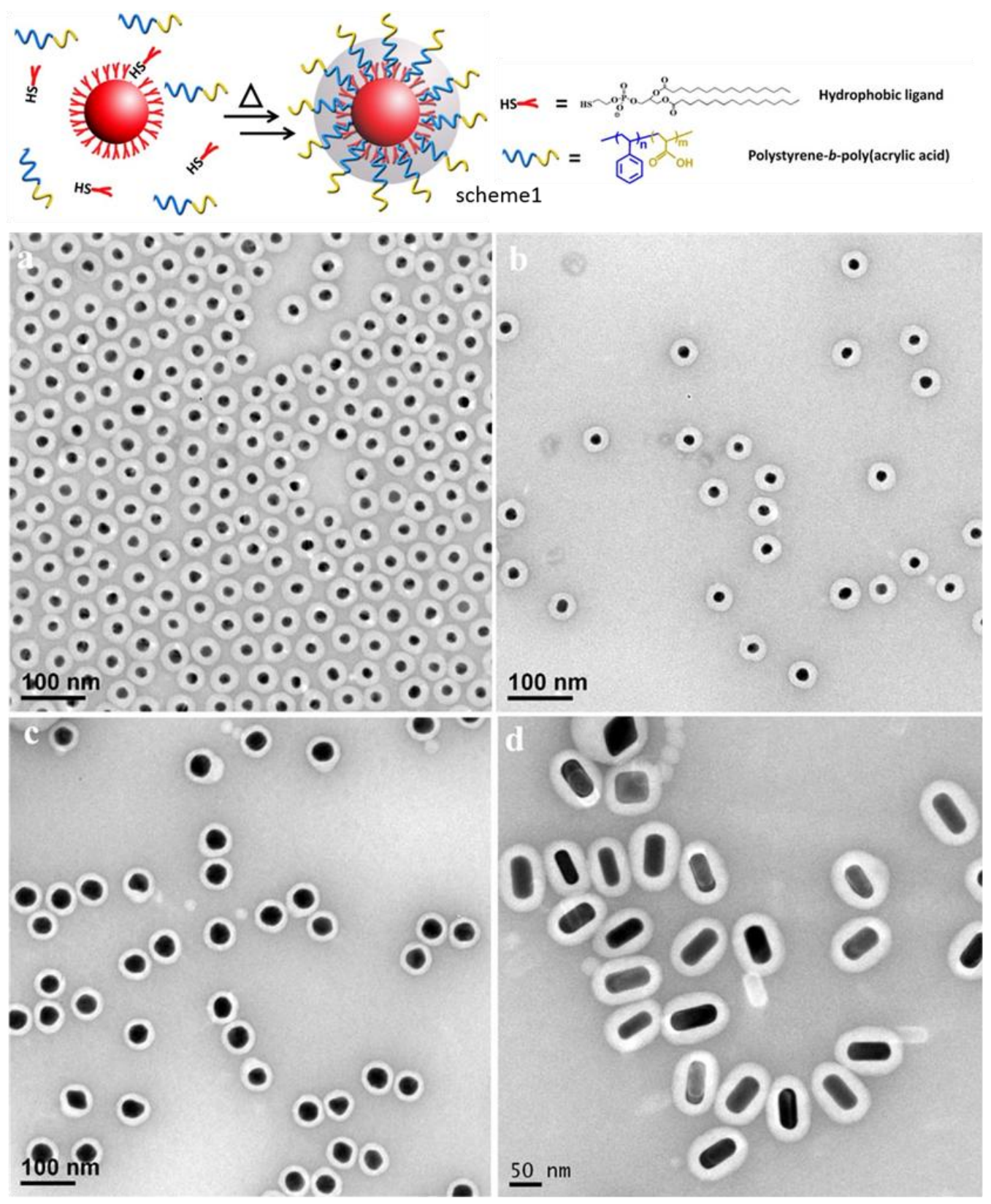

Figure 6.2. Scheme 1 shows the encapsulation of AuNPs with amphiphilic polymers, and TEM images of the different kinds of monomers: (a) $\operatorname{AuNP} @ \operatorname{PSPAA}\left(d_{\mathrm{Au}}=16 \mathrm{~nm}\right.$, mononer type A); (b) AuNP@ $\mathrm{PS}_{144}-b-\mathrm{PAA}_{22}\left(d_{\mathrm{Au}}\right.$ $=16 \mathrm{~nm}$, mononer type $\left.\mathbf{A}^{\prime}\right)(\mathrm{c})$ AuNP@PSPAA $\left(d_{\mathrm{Au}}=32 \mathrm{~nm}\right.$, type $\left.\mathbf{B}\right) ;(\mathrm{d})$ AuNR@PSPAA (type C).

purified by centrifugation, so that the empty PSPAA micelles (without the $\mathrm{Au}$ 
core) formed in the self-assembly can be removed. In a typical "polymerization" process, $16 \mathrm{~nm}$ AuNPs encapsulated in $\mathrm{PS}_{154}-\mathrm{b}-\mathrm{PAA}_{49}$ shells (monomer type A) were used, whereby the monomers dispersed in a DMF-water mixture were treated with acid $(5 \mathrm{mM} \mathrm{HCl})$. After $2 \mathrm{~h}$, the sample was diluted with a large amount of aq. $\mathrm{NaOH}$ to neutralize the acid and remove DMF from the polymer shells, solidifying the polymer shells and locking the assembled chains. The final products were isolated by centrifugation and characterized by transmission electron microscopy (TEM). Typical for the chain growth "polymerization", a large number of monomers remained in the sample (Figure 6.2e), making it difficult to include multiple chains in each image (Figure 6.2e). The monomers can be readily removed in the centrifugation step, where only the ultralong chains were precipitated at a low centrifugation speed. In the following, typically the purified samples are shown in the Figures.

In this system, the $\mathrm{DMF} / \mathrm{H}_{2} \mathrm{O}$ solvent ratio played an important role in controlling the width of the NPs chains. For example, single-line chains of AuNPs were synthesized in $\mathrm{DMF} / \mathrm{H}_{2} \mathrm{O}=6: 1$ mixture; double-line chains were obtained in $\mathrm{DMF} / \mathrm{H}_{2} \mathrm{O}=7: 3$ mixture (Figure 6.2c); and multiple-line chains can be indirectly synthesized by treating the single-line chains in $\mathrm{DMF} / \mathrm{H}_{2} \mathrm{O}=3: 2$ mixture. Here, a higher water content leads to a lesser degree of swelling in the PS domains, which in turn gives a higher PS-solvent interfacial energy (a de-swollen polymer is more dissimilar to the solvent). This leads to a larger 
driving force for the reduction of the surface-to-volume $(\mathrm{S} / \mathrm{V})$ ratio of the polymer cylinder, ${ }^{5 \mathrm{c}}$ favoring thicker chains.

\subsubsection{Effect of surface ligands}

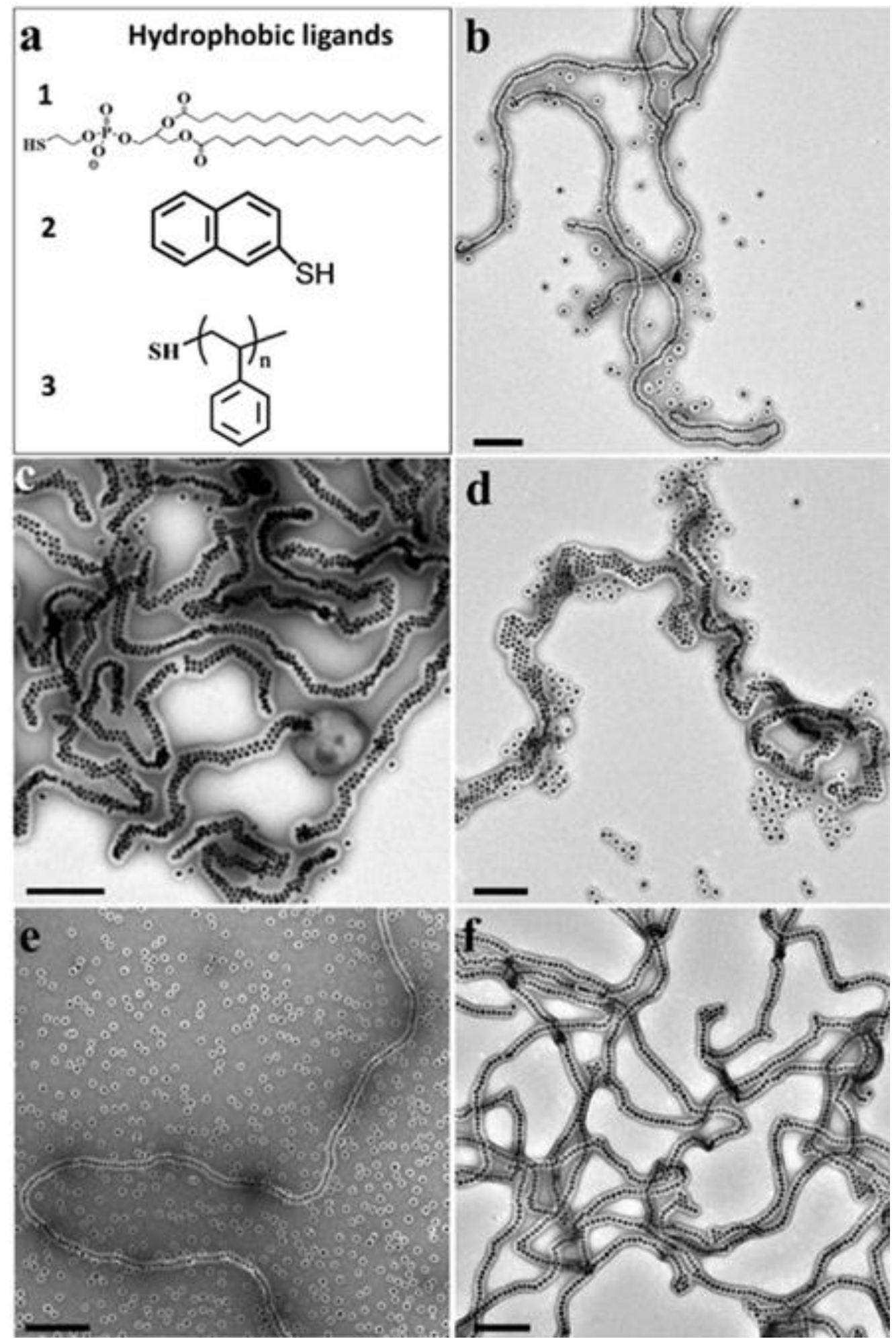


Figure 6.3. (a) Chemical structures of three hydrophobic ligands 1, 2, and 3. (b-d) TEM images of the single-, double-, and multiple-line chains obtained in $\mathrm{DMF} / \mathrm{H}_{2} \mathrm{O}=7: 3$ solution and after purification, where (b) 1-stabilized AuNPs, (c) 2-AuNPs, (d) 3-AuNPs coated in $\mathrm{PS}_{154}-b$-PAA 49 shells were used as the monomers. (e, f) TEM images before and after purification, when (3-AuNP)@ $\mathrm{PS}_{154}-b-\mathrm{PAA}_{49}$ were used as the monomers in $\mathrm{DMF} / \mathrm{H}_{2} \mathrm{O}=6: 1$ solution. Scale bars: $200 \mathrm{~nm}$.

To optimize the conditions for "polymerizing" different types of NPs, we investigated the effects of surface ligands. In $\mathrm{DMF} / \mathrm{H}_{2} \mathrm{O}=7: 3$ mixture, the nature of ligand was found to be of importance for controlling the width of AuNP chains. Previously, double-line AuNP chains were obtained when 1,2-dipalmitoyl-sn-glycero-3-phosphothioethanol (a thiol-ended phospholipid, 2 , Figure 6.3a) was used as the ligand (Figure 6.2c). Under otherwise the identical conditions, when 2-naphtalenethiol (1) was used, single-line AuNP chains were obtained (Figure 6.3b); when thiol-ended polystyrene (3) was used, multi-line AuNP chains were obtained (Figure 6.3d). With the same PSPAA and DMF/ $\mathrm{H}_{2} \mathrm{O}$ ratio, the polymer behavior should be quite similar in these experiments. We speculate that the key factor is perhaps the friction among the ligand-coated AuNPs or the swelling caused by the excess ligand in the reaction mixture. More specifically, in Figure $6.3 \mathrm{~b}$, the polymer had a tendency to contract but the friction among the 1-coated AuNPs might have prevented their reorganization. 


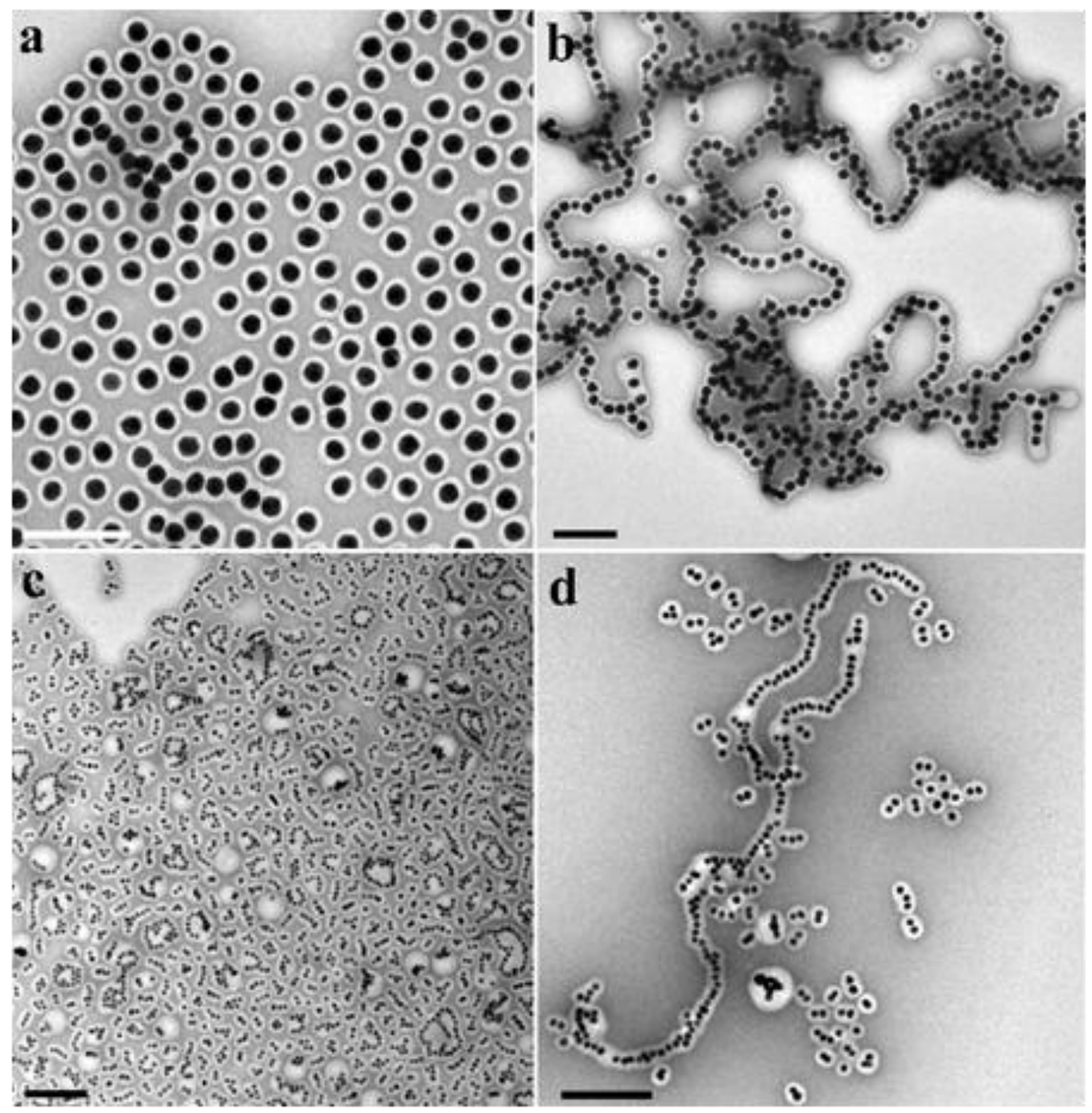

Figure 6.4. TEM images showing the purified products of the "homo-polymerization" when (a) AuNP@ $\mathrm{PS}_{154}-b-\mathrm{PAA}_{49}(d=32 \mathrm{~nm}$, type B) and (b) AuNP@PS $\mathrm{PS}_{144}-b-\mathrm{PAA}_{22}\left(d=32 \mathrm{~nm}\right.$, type $\left.\mathrm{B}^{\prime}\right)$ were used as the monomers in $\mathrm{DMF} / \mathrm{H}_{2} \mathrm{O}=6: 1$ solution; and TEM images showing the products of the "homo-polymerization" of AuNP@ $\mathrm{PS}_{144}-b-\mathrm{PAA}_{22}\left(d=16 \mathrm{~nm}\right.$, type $\left.\mathrm{A}^{\prime}\right)$ in $\mathrm{DMF} / \mathrm{H}_{2} \mathrm{O}=6: 1$ solution: $(\mathrm{c})[\mathrm{HCl}]=5 \mathrm{mM}, 2 \mathrm{~h}$; (d) $[\mathrm{HCl}]=0.25 \mathrm{mM}, 25 \mathrm{~min}$. Scale bars: $200 \mathrm{~nm}$.

Considering the effective stacking among the rigid molecules of 1 , it is 
conceivable that the weaker interactions among $\mathbf{2}$ and the highly flexible PS chains in $\mathbf{3}$ might have facilitated the reorganization of the AuNPs. It is also known that homo-PS can swell the hydrophobic domain of the micelles, reducing the surface density of the PAA blocks (reducing their repulsion) and relaxing the stretching of the PS blocks of PSPAA. ${ }^{7 b, 9}$

To focus on the assembly of NPs, in particular their "copolymerization", we tried to minimize the complexity of the system by avoiding the use of different surface ligands. Fortunately, stable preparative conditions can be established in $\mathrm{DMF} / \mathrm{H}_{2} \mathrm{O}=6: 1$ mixture, where single-line AuNP chains were obtained when any of the three ligands was used (Figure $6.3 \mathrm{f}$ for ligand 3 ). It is likely that the higher degree of swelling by DMF leads to a lower polymer-solvent interfacial energy and thus less driving force for the polymer contraction, reducing the difference in the ligand effects. Hence, we used ligand 2 and $\mathrm{DMF} / \mathrm{H}_{2} \mathrm{O}=6: 1$ mixture in most of the following experiments.

\subsubsection{Effect of PAA length}

The above studies involved only one type of monomers, namely the $16 \mathrm{~nm}$ AuNPs encapsulated in PSPAA shells (monomer type A). If larger AuNPs ( $d=$ $32 \mathrm{~nm}$ ) coated in PSPAA shells (type B) can be adapted for our assembly method, we will have two different types of monomers for their hetero-assembly. However, the larger monomers were not amenable to the same assembly process, giving only short chains (Figure 6.4a). Most of the NPs did not participate in the 


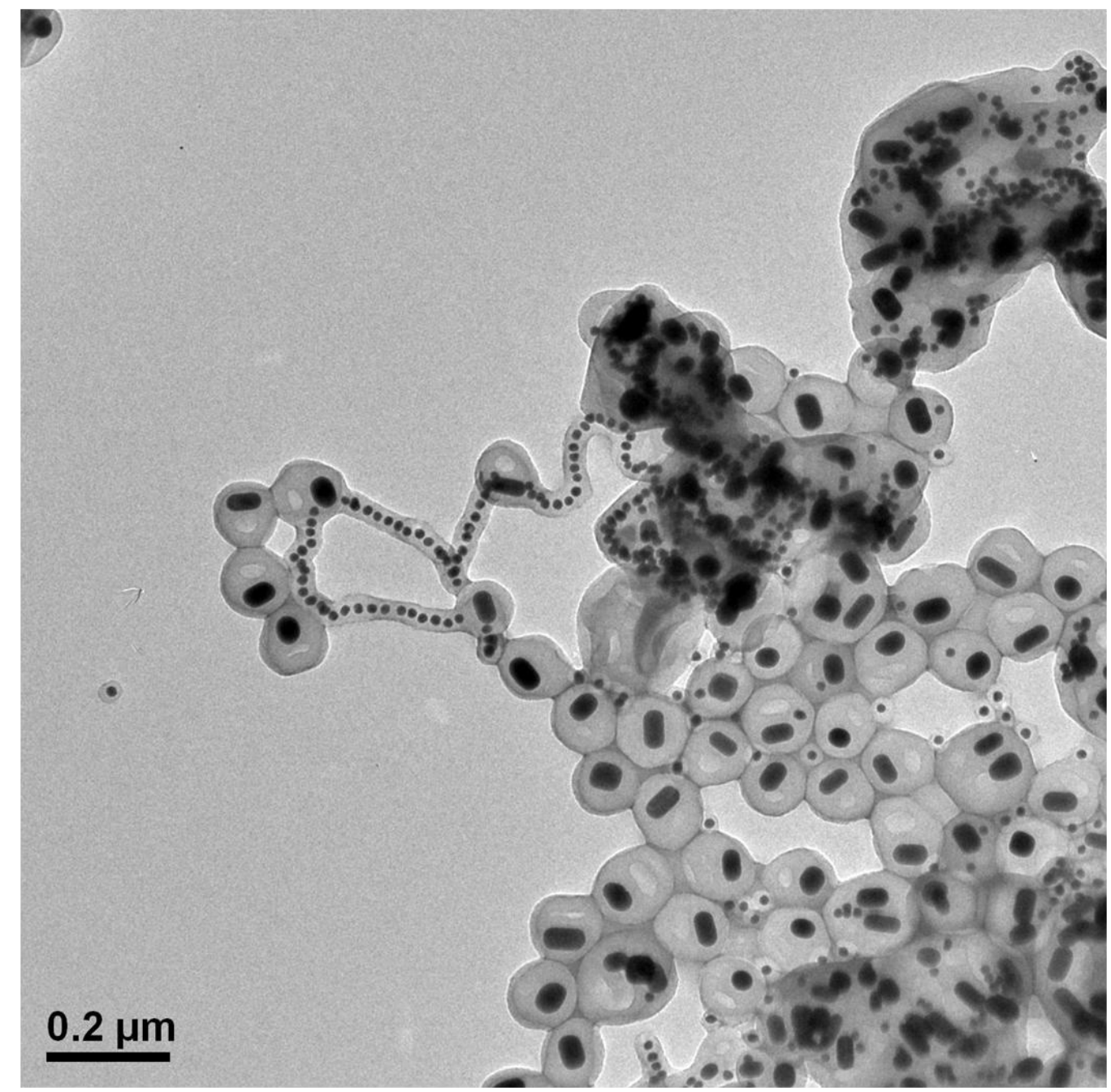

Figure 6.5. TEM images showing the purified products obtained by the "copolymerization" of AuNP@ $\mathrm{PS}_{154}-b-\mathrm{PAA}_{49} \quad$ (type A) and AuNR@PS ${ }_{144}-b-\mathrm{PAA}_{22}$ (type $\left.\mathbf{C}^{\prime}\right)$.

aggregation, indicating a lack of process. Prolonging the assembly time and increasing the NP concentration did not solve the problem. This is likely because that the stronger charge repulsion among the larger monomers greatly reduced the probability of effective collision. Given a same surface charge density, larger particles always have stronger charge repulsion among themselves. ${ }^{1 \mathrm{~d}, 10}$ While using more acid can promote the aggregation, the selectivity of 1D assembly was 
largely compromised, giving vesicles and irregular aggregates (similar to Figure $6.4 c)$.

This problem was eventually solved by using PSPAA with shorter PAA blocks, which under similar conditions would lead to lesser surface charge density. Using $32 \mathrm{~nm}$ AuNPs encapsulated in $\mathrm{PS}_{144}-\mathrm{b}-\mathrm{PAA}_{22}$ as monomers (type $\mathrm{B}^{\prime}$ ), long chains of the AuNPs were obtained under the same preparative conditions (Figure 6.4b). Obviously, the aggregation was more extensive than that in Figure 3a. Because the NP concentration was the same, the probability of collision should be similar. Thus, the new polymer likely improved the probability of effective collision.

For $16 \mathrm{~nm}$ AuNPs in $\mathrm{PS}_{144}$-b-PAA 22 shells (type $\mathrm{A}^{\prime}$ ), the same treatment led to rapid aggregation of the monomers, giving rise to small clusters that were typical of step-growth mode (Figure 6.4c). As discussed above, the depletion of monomers indicates that all monomers can participate in the aggregation, as opposed to the chain-growth mode where most of them cannot. Hence, the reduction in the barrier for collision likely also reduced the selectivity of the chain growth mode, compromising the synthetic control. Further control experiments showed that, by lowering the acid concentration and shortening the assembly time, chains of A' can still be prepared (Figure 6.4d). The large number of dimers and trimers in this sample indicated that the aggregation was still less selective than those in Figure 6.4. Nevertheless, this understanding may provide a new pathway for synthesizing dimers and trimers ${ }^{11}$ for applications in 
surface-enhanced Raman scattering (SERS). ${ }^{12}$

Given the faster assembly of $\mathrm{A}^{\prime}$ and $\mathrm{B}^{\prime}$ than that of $\mathrm{A}$ and $\mathrm{B}$, the shorter PAA chains were of critical importance in promoting the effective collision, supporting our previous hypothesis in micelle aggregation. ${ }^{5 \mathrm{c}}$

\subsubsection{Selection of the preparative conditions}

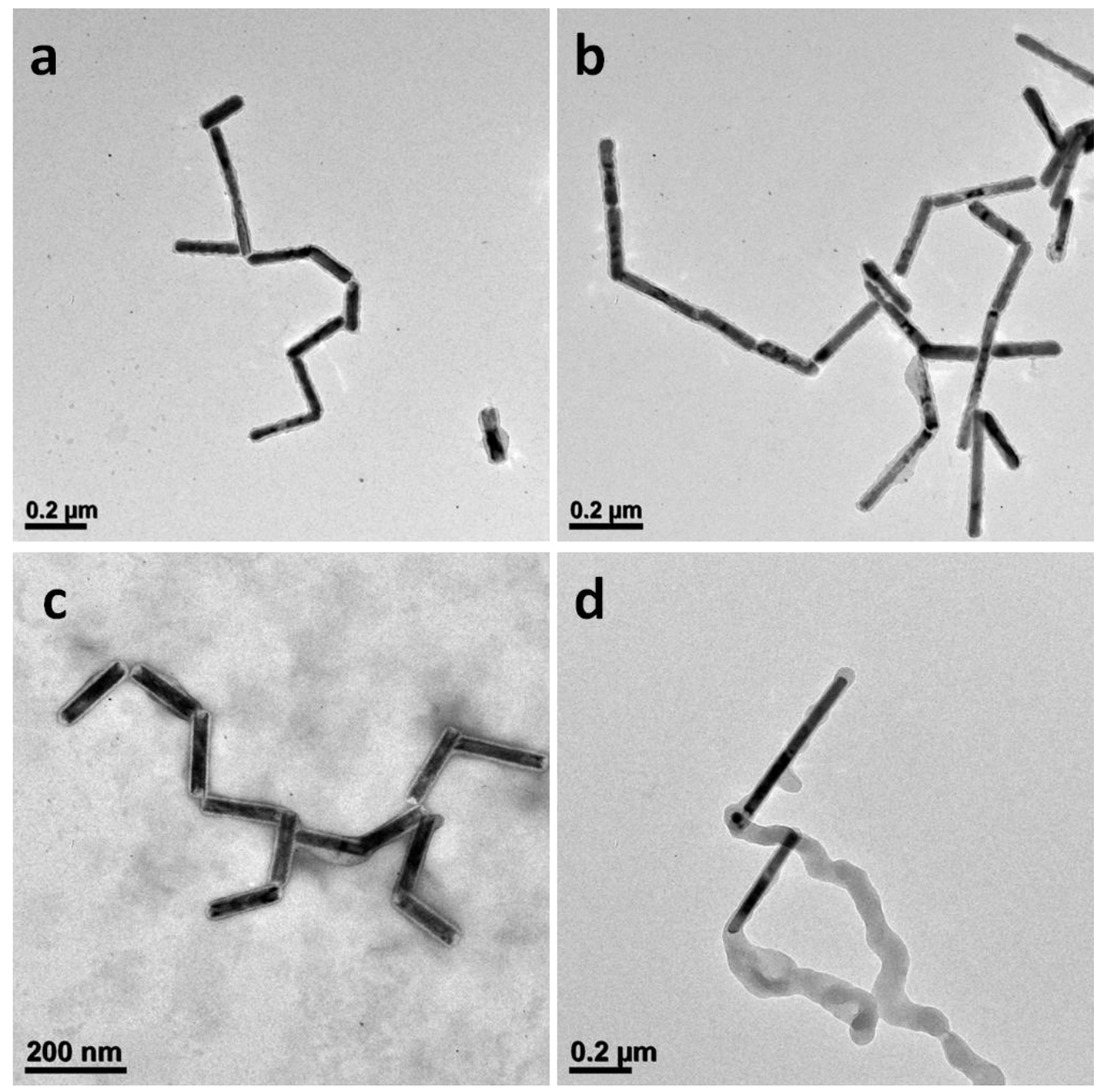

Figure 6.6 TEM images showing the purified products obtained by the

"homopolymerization” of (a-c) Te NWs@PSPAA (type D) linear chain; and(d)

the "copolymerization" of Te NWs@PSPAA and PSPAA micelles (D- $b$-E).

In summary, in this system there are quite a few controlling factors, including 
the solvent ratio, surface ligand, polymer chain length, acidity, temperature, and assembly time. Obviously, search by trial and error within the 6-dimension parameter space would be very difficult, if not totally impossible. Rational design is thus of crucial importance.

The solvent ratio affects the swelling of the polymer domain, whereby a higher DMF ratio leads to a higher degree of swelling, and thus a higher mobility of the polymer in their reorganization. ${ }^{8}$ Regarding to the surface ligands, their roles are complex in affecting the interplay between the NP and the polymer shell, as well as the interplay among the NPs in the aggregated clusters. Such a topic is of interest but beyond the scope of this work. Regarding to the polymer chain length, the complex effects have been previously discussed. ${ }^{13}$ In this work, only the PAA length was modulated (shortened) to facilitate NP aggregation. Regarding to acidity, a higher acidity leads to a higher degree of protonation of the PAA chains, reducing charge repulsion. This affects both the kinetic barrier of aggregation (i.e., charge repulsion, vide supra) and the thermodynamic end point of polymer morphology. ${ }^{5 a, 14}$ In this work, a same amount of acid was used to reduce the complexity of the system. Regarding to the temperature, a higher temperature leads to a higher kinetic energy, facilitating the reorganization of both the polymer chains in the micelles and the NPs in the clusters. At high temperature (> $90{ }^{\circ} \mathrm{C}$ ), the polymer micelles quickly evolve towards vesicles, complicating the assembly and purification of NPs. Hence, the temperature was optimized at $60{ }^{\circ} \mathrm{C}$. 


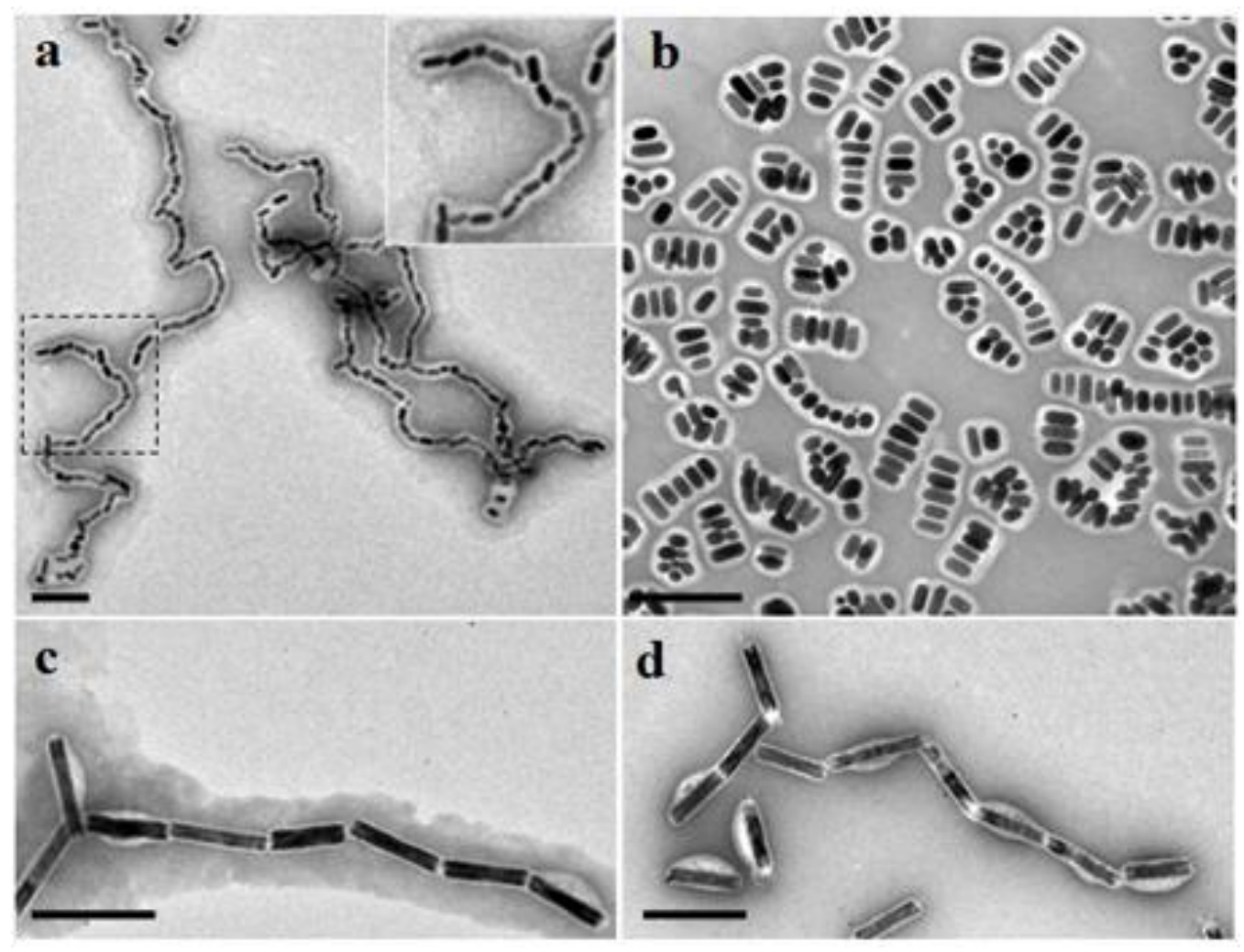

Figure 6.7. (a, b) TEM images showing the purified products obtained by the "homo-polymerization" of AuNR@PSPAA, where 2 (a) and 3 (b) were used as the ligands for the AuNRs. (c, d) TEM images of the purified products of the "homo-polymerization" when the TeNW@PSPAA (type D) were used as the monomers. Scale bars: $200 \mathrm{~nm}$. Reprinted with permission from ref. [59], copyright 2014 American Chemical Society.

Regarding to the assembly time, it affects not only the aggregation of the NPs1d and the structural reorganization of the resulting clusters, ${ }^{15}$ but also the transformation of the micelles from spheres to cylinders and then to vesicles. ${ }^{16}$

On the basis of these understandings, we optimized the conditions as follows. The solvent ratio $\left(\mathrm{DMF} / \mathrm{H}_{2} \mathrm{O}=6: 1\right)$ and the temperature $\left(60{ }^{\circ} \mathrm{C}\right)$ were kept at constant in our experiments. Ligand 2 was typically used for consistency. 
$\mathrm{PS}_{144}$-b-PAA 22 was used to promote the monomer aggregation when needed. Acidity (usually $5 \mathrm{mM} \mathrm{HCl}$ ) and assembly time (typically $2 \mathrm{~h}$ ) were used as the variables.

\subsection{4 "Homo-polymers" of nanorods and nanowires}

Among the various types of nanostructures known in the literature, $\mathrm{Au}$ nanorods (NRs) have a unique shape that can be easily distinguished from the AuNPs. AuNRs were typically synthesized using hexadecyltrimethylammonium bromide (CTAB) as the surfactant. Being a positively charged amphiphilic molecule, it can interfere with the self-assembly of amphiphilic PSPAA. Thus, the as-synthesized AuNRs with high CTAB concentration was purified twice using centrifugation, before they were used for the PSPAA encapsulation. The sample was then further purified by centrifugation to remove the empty micelles.

The resulting core-shell NPs $\left(A u N R @ P_{154}-b-P A A_{49}\right.$, with ligand 2 as the surface ligand) were used as the monomer C. "Homo-polymerization" of C gave chains of AuNRs as shown in Figure 6.4a, where most of the AuNRs were aligned end-to-end along the longitudinal direction. Only in a few cases the neighboring AuNRs were arranged in end-to-side configuration. This end-to-end selectivity is likely due to (a) the higher curvature of the polymer domain at the ends, which leads to higher activity of the polymer, ${ }^{17}$ and (b) the lesser charge repulsion of the end-to-end aggregation than that of end-to-side aggregation.1d Interestingly, when ligand $\mathbf{3}$ was used for making the monomer $\mathrm{C}$, 
"homo-polymerization" led to the step-growth mode, giving small clusters with

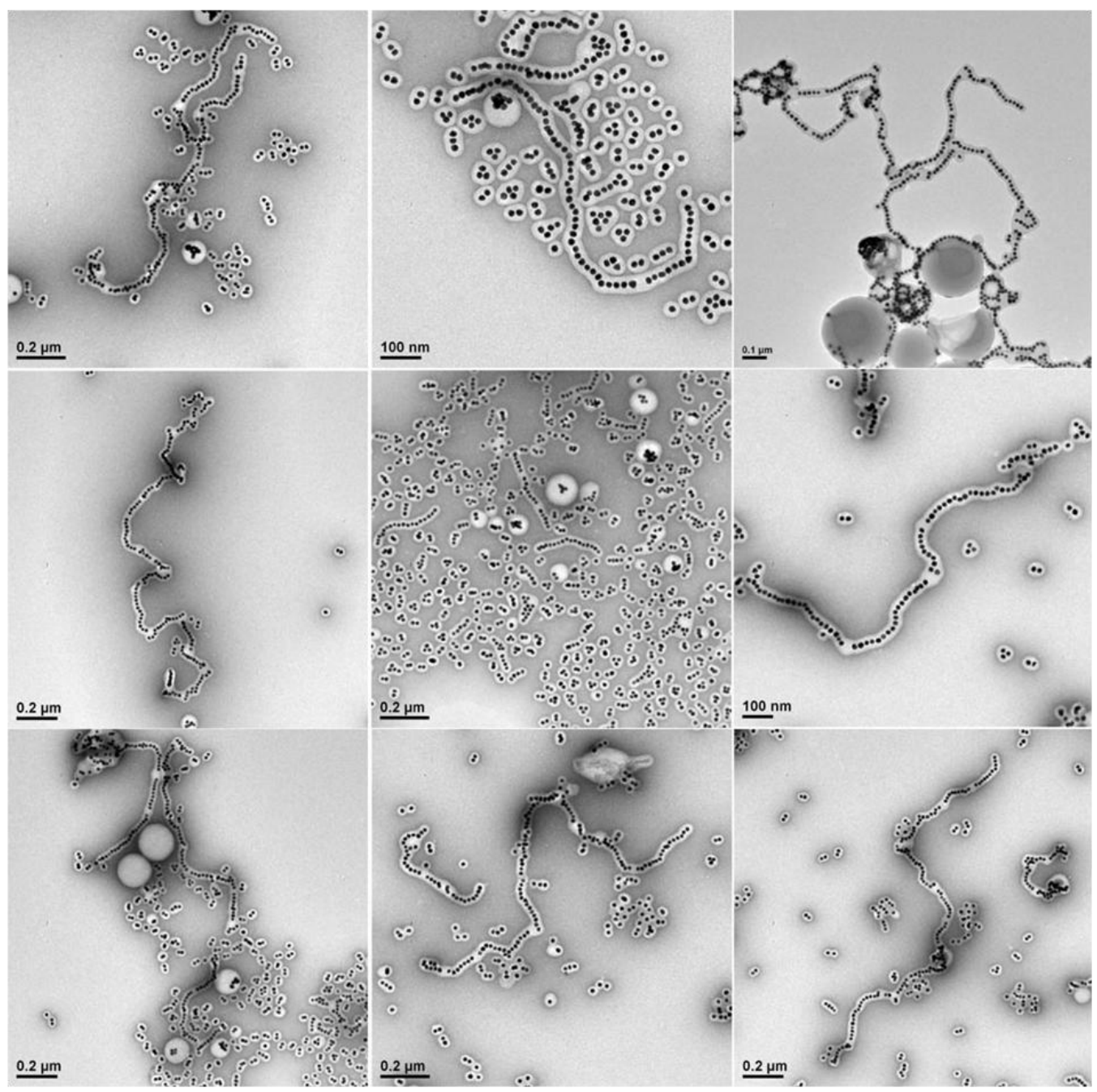

Figure 6.8. TEM images showing the purified products when

AuNP@PS ${ }_{144}-\mathrm{PAA}_{22}$ (type $\mathbf{A}^{\prime}$ ) were used in "home-polymerization", where the monomers were dispersed in $\mathrm{V}_{\mathrm{DMF}}: \mathrm{V}_{\mathrm{H} 2 \mathrm{O}}=6: 1$ and $\mathrm{HCl}(0.25 \mathrm{mM})$, and heated at $60{ }^{\circ} \mathrm{C}$ for $25 \mathrm{~min}$.

mostly side-to-side configuration (Figure 6.4b). Comparing to the results in Figure 6.2d, in the presence of $\mathbf{3}$ the PSPAA micelles probably preferred a larger width, allowing the embedded AuNRs to adopt the perpendicular configuration.

We selected TeNWs as a longer alternative to the AuNRs. They were about 50 
$\mathrm{nm}$ in diameter and $180 \mathrm{~nm}$ in length (Figure 6.4c). The as-synthesized TeNWs were purified by centrifugation to remove the surfactant sodium dodecylsulfate (SDS) used in the synthesis. ${ }^{18}$ They were then encapsulated in PSPAA shells by incubating them in $\mathrm{DMF} / \mathrm{H}_{2} \mathrm{O}=6: 1$ mixture, where PSPAA can directly self-assembly on their hydrophobic surface without any additional ligand. After purification, the resulting TeNW@ $\mathrm{PS}_{154}-\mathrm{b}-\mathrm{PAA}_{49}$ was used as the monomer D. Upon acid treatment, the TeNWs assembled into chains with selective end-to-end configuration (Figure 6.4c-d).

\subsubsection{Random "copolymers" of NPs}

With two types of monomers $\mathrm{A}$ and $\mathrm{B}$, we now investigate their "copolymerization". Directly mixing the monomers in the process (the concentration ratio $[\mathrm{A}]:[\mathrm{B}]$ was roughly $1: 1$, in the number of the NPs) gave a random "copolymer" (A-r-B, Figure 6.5a). Only a few B NPs were incorporated in the chains (the incorporated ratio $\mathrm{A}: \mathrm{B}=61: 1$ ), and the remaining monomers contained mostly B NPs. Given the roughly equal concentration of A and B, this result further indicates that the larger B NPs have a lower tendency towards aggregation. After differential centrifugation to remove the monomers, the low content of B in the resulting "copolymer" is more evident as shown in Figure 5b.

To increase the $\mathrm{B}$ content, $\mathrm{B}^{\prime}$ was used in the place of $\mathrm{B}$ for the “copolymerization". That is, A and B' were used in a same solution, even though the monomers have different kinds of polymer shells. It was previously known 

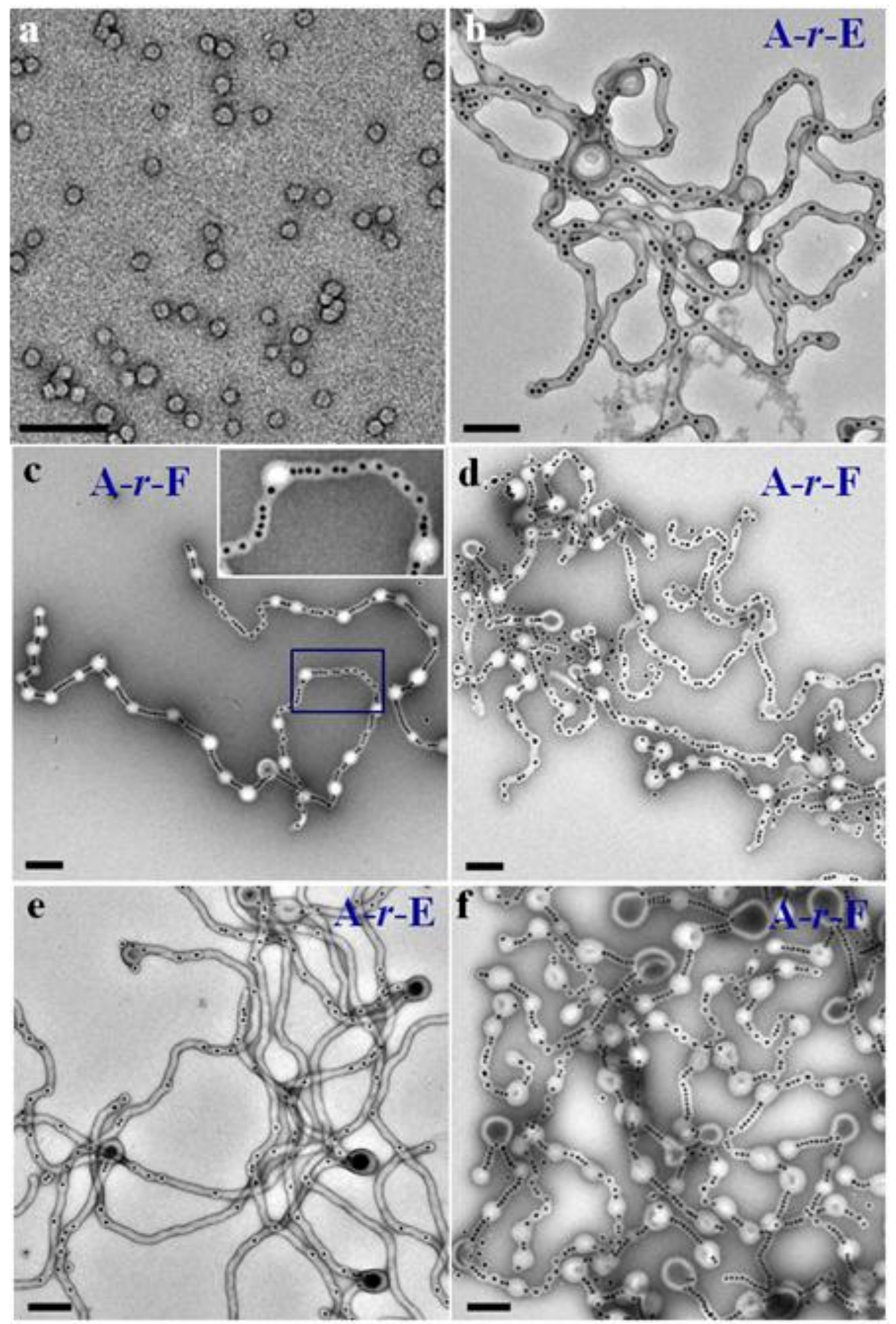

Figure 6.9. TEM images showing the resulting random "copolymers" synthesized from $(\mathrm{a}, \mathrm{b})$ monomer $\mathrm{A}$ and $\mathrm{B}([\mathrm{A}] /[\mathrm{B}]=1: 1)$, before and after purification, respectively; (c, d) monomer $A$ and $\mathrm{B}^{\prime}\left([\mathrm{A}] /\left[\mathrm{B}^{\prime}\right]=1: 1\right)$, before and 
after purification, respectively; (e) monomer $\mathrm{A}$ and $\mathrm{B}^{\prime},[\mathrm{A}] /\left[\mathrm{B}^{\prime}\right]=1: 2$; (f) monomer A and B', $[\mathrm{A}] /\left[\mathrm{B}^{\prime}\right]=2: 1$. Scale bars: $200 \mathrm{~nm}$. Reprinted with permission from ref. [59], copyright 2014 American Chemical Society.

that these PSPAA molecules do not readily exchange among the micelles (i.e., the dissolution-remicellization process is negligible ${ }^{17 b, 19}$ ). As shown in Figure $6.5 \mathrm{c}, \mathrm{d}$, when the ratio of the initial concentration was $[\mathrm{A}]:\left[\mathrm{B}^{\prime}\right]=1: 1$, the "copolymerization" went well. After chain formation, a significant amount of monomers remained in the sample, indicating that the chain growth mode was still followed (Figure 6.5c). In contrast to Figure 6.5b, the higher reactivity of B' led to its higher content in the resulting A-r-B' chains (Figure 6.5d, A:B' $=3.5: 1$ ).

The A/B' ratio can be readily tuned by varying the concentration ratio of the monomers. As expected for a random "copolymer", when the reactivity of the monomers is kept as constant, their probability of being incorporated in the "copolymer" should be dependent on their concentration. To test this trend, the following control experiments were carried out. When the $[\mathrm{A}]$ : $\left[\mathrm{B}^{\prime}\right]$ concentration ratio was decreased from 1:1 to $1: 2$, the resulting "copolymer" had a higher B' content (Figure $6.5 \mathrm{e}, \mathrm{A}: \mathrm{B}^{\prime}=1.4: 1$ ). On the other hand, when the $[\mathrm{A}]:\left[\mathrm{B}^{\prime}\right]$ ratio was increased to $2: 1$, the content of $\mathrm{B}$ ' in the "copolymer" chains was dramatically decreased (Figure 6.5f, A:B' = 20:1).

Figure 6.6a shows the result after "co-polymerizing" $\mathrm{A}$ and $\mathrm{C}$ (random "copolymer" A-r-C, A:C = 42:1). Only a few AuNRs were incorporated in the 
resulting "copolymer" and they often align end-to-end with the neighboring

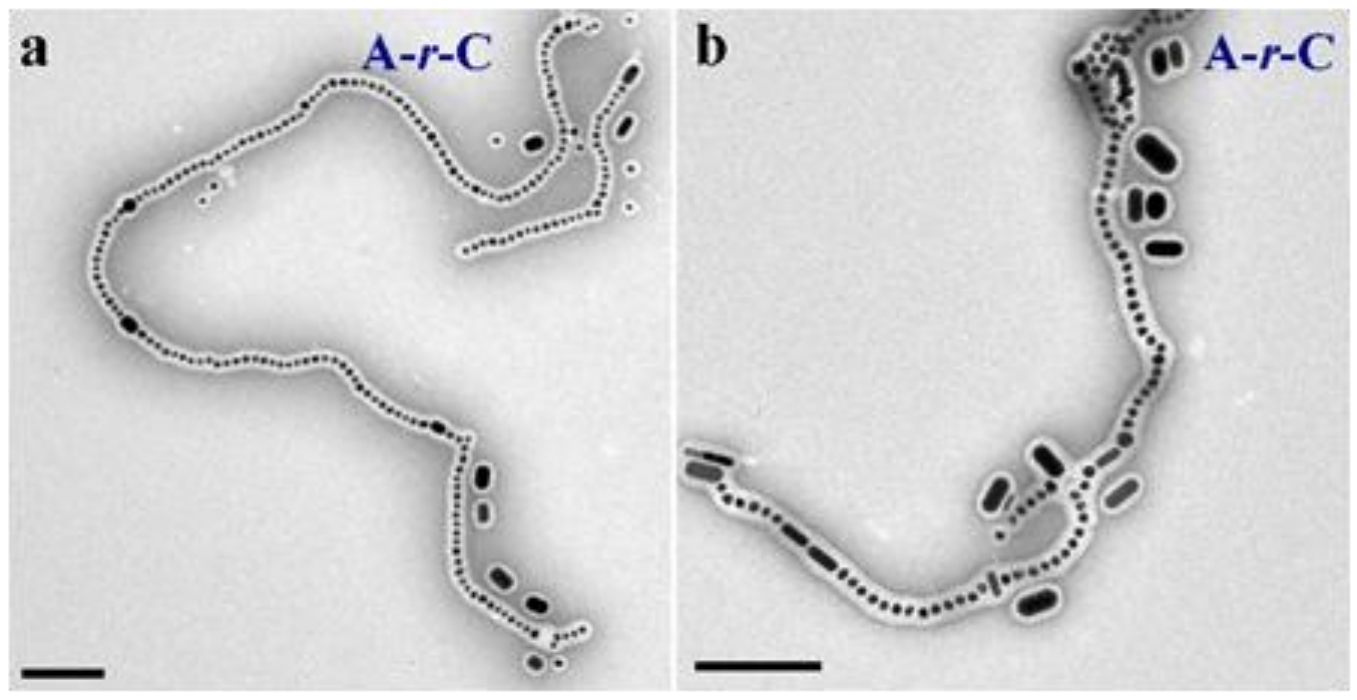

Figure 6.10. TEM images showing the random chains $(\mathrm{A}-r-\mathrm{C})$ synthesized via the "copolymerization" of monomer A and C: (a) ligand 2 and (b) ligand $\mathbf{3}$, respectively, were used as the surface ligands for C. Scale bars: $200 \mathrm{~nm}$.

AuNPs. However, our efforts to reduce the A:C ratio was not successful. While the AuNRs can be encapsulated in $\mathrm{PS}_{144}-\mathrm{b}-\mathrm{PAA}_{22}$ (monomer $\mathrm{C}^{\prime}$ ), we were not able to use these more reactive monomers for our method. ${ }^{20}$ Upon acid treatment, most of the monomers turned into vesicles and few participated in the "polymerization", likely due to the interference by the residue CTAB.

An initial attempt was carried out to study the self-assembly in a more complex system using different ligands. The monomers $\mathrm{C}$ were prepared using $\mathbf{3}$ as the ligand; whereas monomers A contained $\mathbf{2}$ as the ligand (both used $\mathrm{PS}_{154}$-b-PAA 49 ). Mixing them in the synthesis gave long chains where most of the AuNRs adopted end-to-end configuration with the neighboring NPs (Figure 
6b), ${ }^{21}$ although a few cases of end-to-side attachment can also be observed.

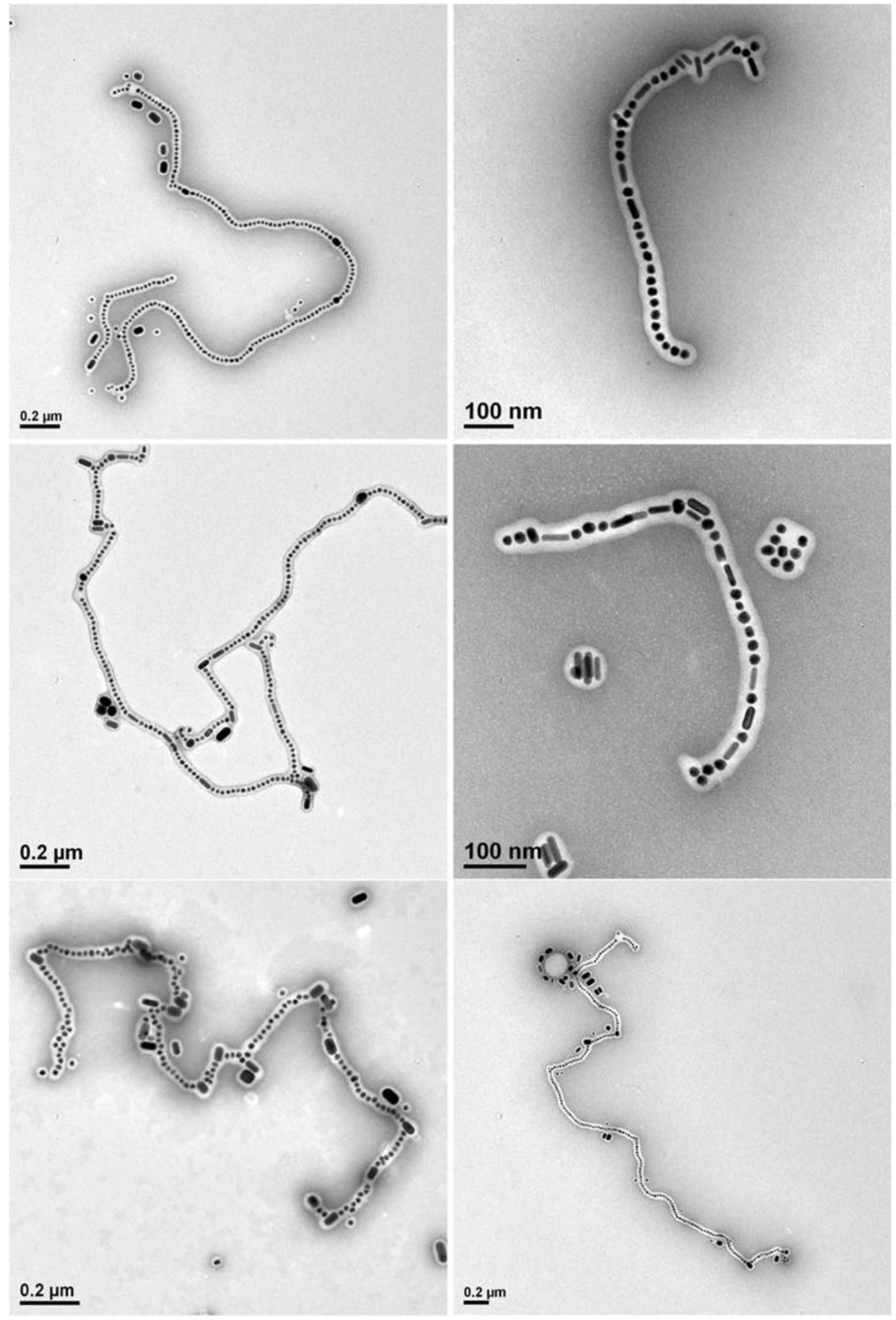

Figure 6.11. TEM images showing the purified products obtained by the “copolymerization” of AuNP@PSPAA (type A) and AuNR@PSPAA (type C). 
Given the slow dissolution-remicellization process of PSPAA, there was probably negligible ligand exchange among the AuNPs and AuNRs. This result is of interest because side-to-side aggregation dominated when only monomers $\mathrm{C}$ were used for the polymerization (Figure 6.4b). Indeed, there were two modes even in the same sample: the AuNRs were side-to-side when aggregating with each other, but end-to-end when aggregating with the AuNPs (Figure 6.6b). ${ }^{21}$

\subsection{6 "Copolymers" of NPs with cylinders/vesicles}

In comparison to the AuNPs and AuNRs, PSPAA nano-structures such as cylinders and vesicles can be more easily incorporated into the "copolymers". A simplest method is to add extra $\mathrm{PS}_{154}$-b-PAA $49\left(2.0 \times 10^{-6} \mathrm{mmol}\right)$ during the "polymerization" of monomer A. The extra PSPAA are usually in the form of spherical micelles (Figure 6.7a); upon acid treatment they can aggregate and transform to cylindrical micelles, ${ }^{5 a, 5 \mathrm{c}, 6}$ which can be considered as another kind of monomers (type E). Thus, "copolymers" of AuNPs with PSPAA cylinders (A-r-E) can be obtained ( $5 \mathrm{mM} \mathrm{HCl}, 1.5 \mathrm{~h}$ ), where both A and $\mathrm{E}$ are randomly incorporated in the resulting chain (Figure 6.7b).

It is well known that PSPAA cylinders can transform to vesicles, where the reduction of the surface to volume ratio $(\mathrm{S} / \mathrm{V})$ of the micellar structures provides the thermodynamic driving force. ${ }^{5 c, 8}$ Higher temperature, longer incubation time and more acid are known to promote the structural transformation of the micelles. Two control experiments similar to Figure $6.7 \mathrm{~b}$ were carried out. In the first one, 
the reaction mixture was incubated for a longer period $(5 \mathrm{mM} \mathrm{HCl}, 6 \mathrm{~h}$, Figure

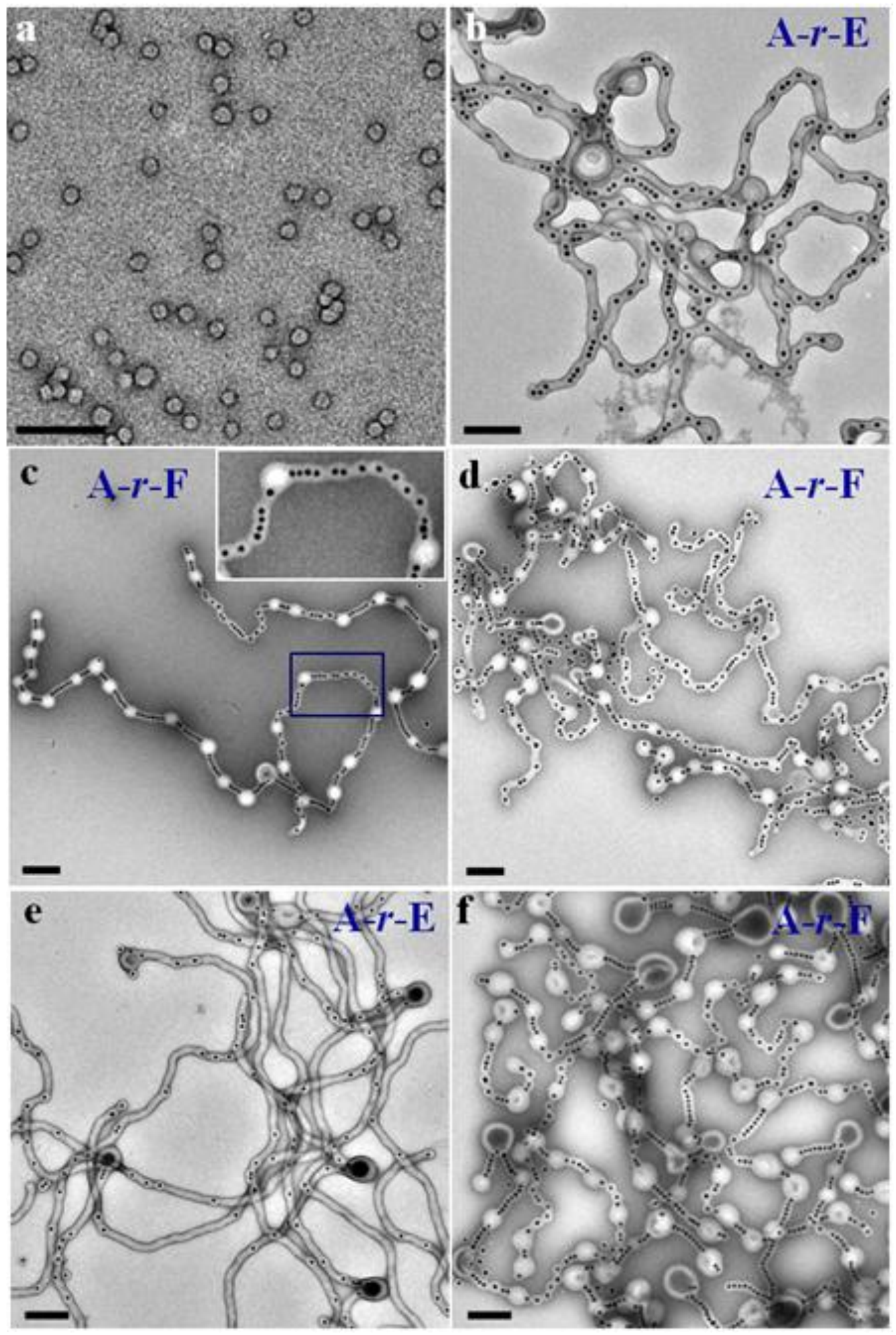

Figure 6.12. (a) TEM images of PSPAA spherical micelles. TEM images showing the purified random "copolymer" chains consisted of AuNP chains and 
PSPAA domains, which were obtained at: $(\mathrm{b}-\mathrm{c}) 2.0 \times 10^{-6}$ mmol PSPAA, $[\mathrm{HCl}]=$ $5 \mathrm{mM}, t=1.5$, and $6 \mathrm{~h}$, respectively; (d) $2.0 \times 10^{-6} \mathrm{mmol}$ PSPAA, $[\mathrm{HCl}]=9 \mathrm{mM}$, $t=1.5 \mathrm{~h} ;(\mathrm{e}-\mathrm{f}) 4.0 \times 10^{-6} \mathrm{mmol}$ PSPAA micelles, $[\mathrm{HCl}]=5 \mathrm{mM}, t=3$ and $6 \mathrm{~h}$, respectively. Scale bars: $200 \mathrm{~nm}$.

6.7c), whereas in the second experiment, more acid was added in the reaction mixture $(9 \mathrm{mM} \mathrm{HCl}, 1.5 \mathrm{~h}$, Figure $6.7 \mathrm{~d})$. In the resulting samples, most of the cylindrical PSPAA domains turned into vesicles. Only very short cylindrical sections remained (inset of Figure 6.7c), probably due to insufficient material to make vesicles. The vesicular moieties can be viewed as yet another type of monomer units (type F), making the composite chains "copolymers" of AuNPs and vesicles (A-r-F).

However, prefabricated PSPAA vesicles cannot be directly used as the monomers for the "copolymerization". We synthesized PSPAA vesicles of about $200 \mathrm{~nm}$ in diameter using a previously reported method. ${ }^{5 \mathrm{c}}$ After purification, these vesicles were mixed with $\mathrm{A}$ and they were subjected to the "copolymerization" conditions. Only "homopolymers" of A were obtained and very few vesicles participated in the assembly. ${ }^{20}$ We speculate that the vesicles are less prone to the aggregation process because they are large and thermodynamically stable.

The random copolymer of A-r-E can be easily tuned by varying the ratio of A to the extra PSPAA micelles. When the extra PSPAA amount was doubled $(4.0 \times$ 
$10^{-6} \mathrm{mmol}$ ), the average cylinder length was obviously increased in the resulting chain (Figure 6.7e). After prolonged incubation $(6 \mathrm{~h})$, this sample turned into random "copolymer" A-r-F, where most of the cylindrical sections were converted to vesicles (Figure 6.7f). The resulting vesicular moieties were polydispersed in size, but they were in general larger than those in Figure 6.7c. The wide size distribution of the vesicles is probably a result of the wide length distribution of the cylindrical sections in the initial step.

The conversion of cylindrical to vesicular micelles has been previously studied by our group. ${ }^{8}$ With the inserted AuNPs serving as markers here, the selective conversion of the cylindrical sections provides additional evidence that the process can proceed without external input of materials, or via dramatic restructuring of the micelles. The results thus support our previously proposed kinetic pathway for the cylinder-to-vesicle transformation.

\subsection{7 "Block copolymer" of NWs with NPs}

To fabricated longer building blocks for the "copolymerization", we used CNTs and TeNWs with large aspect ratio $(1=500 \mathrm{~nm} ; \mathrm{d}=50 \mathrm{~nm})$. CNT bundles of about $20 \mathrm{~nm}$ in width and 0.3-5 $\mathrm{nm}$ in length were dispersed in DMF and then encapsulated in $\mathrm{PS}_{154}$-b-PAA 49 shells $(\mathrm{CNT} @ \mathrm{PSPAA}$, monomer G, Figure 6.8a). ${ }^{21}$ After mixing with A, acid was added to induce "copolymerization". "Block copolymers" of A-b-G were obtained (Figure 6.8b), though with a low yield. Among the products, there was a significant amount of "homo-polymers" 
of A. During the process, the collision between $\mathrm{G}$ and $\mathrm{A}$ is much less probable
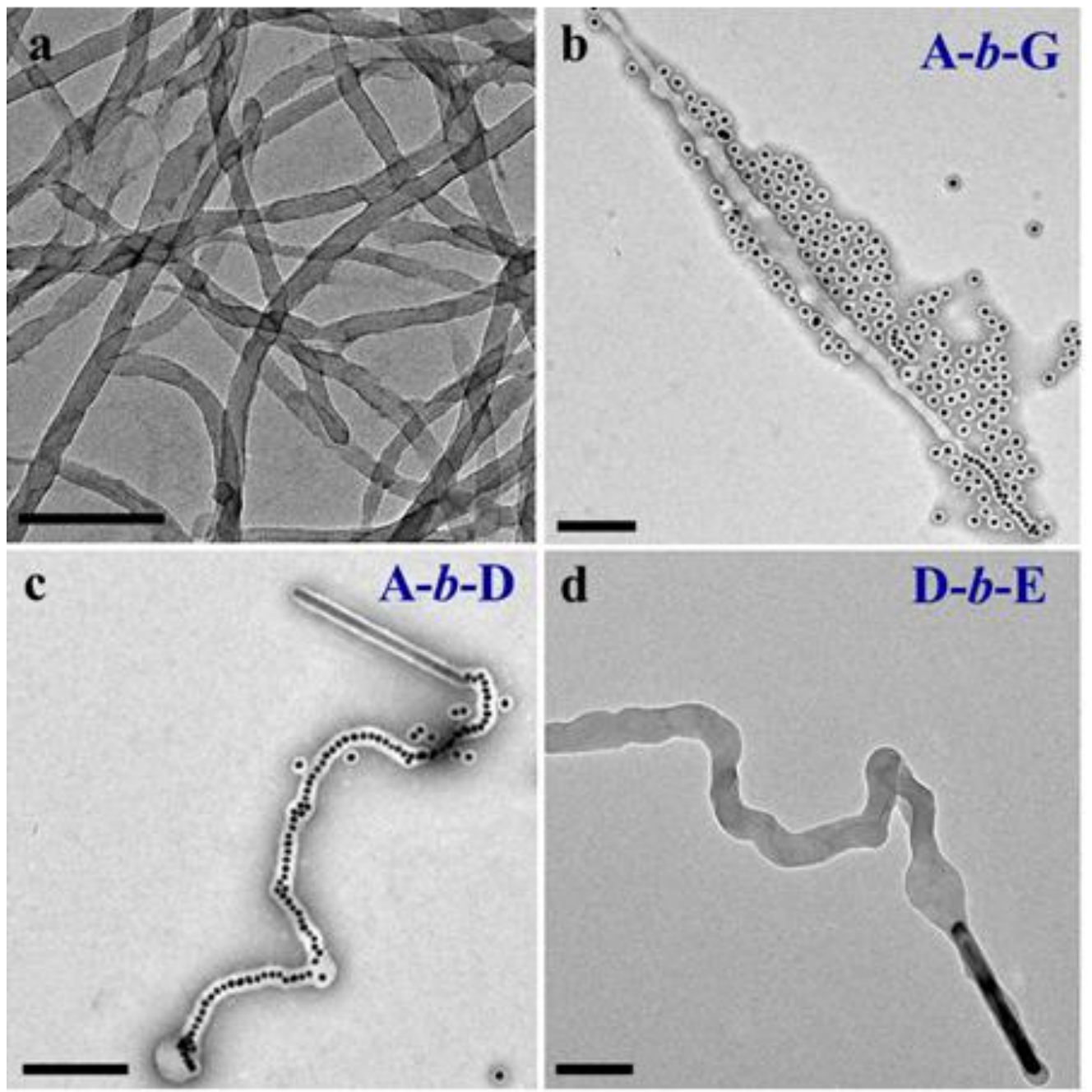

Figure 6.13. TEM images showing (a) CNT@PSPAA monomers (type G); (b)

the block copolymer chains obtained via the "copolymerization" of A and G; (c, d) the block "copolymer" chains obtained via the "copolymerization" of (c) A with D; and (d) D with E. Scale bars: $200 \mathrm{~nm}$.

than the collision between $\mathrm{A}$ and $\mathrm{A}$, due to the difference in their concentration. Though the tip of G appears to be sharp, with a much higher curvature than its side, it is not comparable to the curvature of the PSPAA shells on the small AuNPs. 
Similarly, TeNW@PSPAA (monomer D) was also used for the “copolymerization", giving block “copolymers" A-b-D (Figure 6.8c). “Copolymerizing” D with extra spherical micelles (E) gave block "copolymers" D-b-E (Figure 6.8d).

In all these examples, the chain growth always occurred on only one end of the existing block (CNT or TeNW). Typically, in the chain growth polymerization mode, most of the monomers are inactive, whereas a few are active enough to undergo extensive polymerization into long chains. ${ }^{2 \mathrm{~b}}$ In other words, only a small percentage of the monomers were activated. Considering this low probability of activation, simultaneously activate both ends of the CNT@PSPAA or TeNW@PSPAA is highly improbable.

Thus, there is a dilemma in the synthetic design. Making the chain growth less selective (such as in Figure 6.13d) would lead to the step growth mode. The resulting "homopolymers" of A will compete with the addition of NPs to the CNT@PSPAA, limiting the length of the block "copolymers". On the other hand, making the chain growth more selective would reduce the probability of activating the ends of the CNT@PSPAA, leading to a larger percentage of unreacted CNT@PSPAA and a lower yield of the block "copolymers".

\subsubsection{Random versus block "copolymers"}

Our attempts to synthesize "block copolymers" directly from the smaller monomers (AuNPs and AuNRs) were largely unsuccessful. In one of our 
attempts, AuNRs were first "polymerized" into chains and purified to remove most of the remaining monomers. The products were then mixed with monomers A to grow a second block. However, "homo-polymerization" of A dominated the reaction and only short block of A was found in a few cases of A-b-C. ${ }^{20}$ Attempts to use the side-to-side aggregated AuNRs were also unsuccessful.

Initially, we spent great efforts optimizing the polymerization conditions, hoping to fine-tune the reactivity of the monomers via changes in ligand, PSPAA, or solvent conditions. However, systematic analysis of the system showed that there is no simple solution. There are major differences between the syntheses of random and block "copolymers" as shown in figure 6.10 and figure 6.11. The former is a one-step process where the growing end remains active after each monomer addition, regardless of the type of the monomer. After reaction, the remaining monomers and short chains can be easily removed by differential centrifugation. In contrast, the synthesis of block "copolymers" requires a two-step process, where the purification after the first step is essential for removing the excess monomers as shown in figure 6.12 and figure 6.13. To completely exhaust the monomers in the first step reaction is not viable because the monomer concentration would be too low to complete the reaction in meaningful time frames. After the purification, however, there is a major problem in selectively activating the ends of the "polymer" for the second step growth, as discussed in the above section. 
In the conventional polymerization methods, both free radicals and initiating functional groups can induce the chain growth polymerization mode. While free

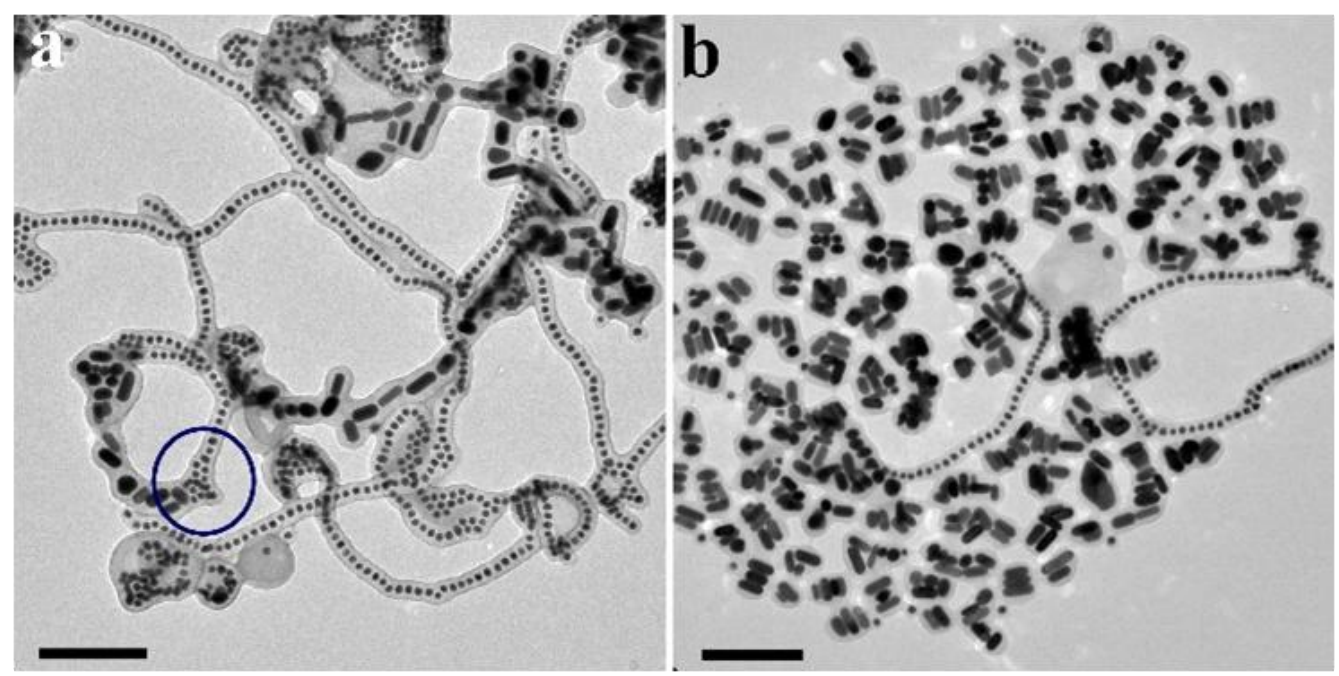

Figure 6.14. TEM images showing the purified products when the prefabricated

AuNR chains were used for growing monomers $\mathbf{A}$ and then random with block polymer: (a) end-to-end AuNR aggregates using 2 as the ligand; (b) side-to-side AuNR aggregates using $\mathbf{3}$ as the ligand. Scale bar: $200 \mathrm{~nm}$.

radicals can be used for making random copolymers, they are not the best choice for making block copolymers, which are typically synthesized by living polymerization. ${ }^{22}$ Basically, the free radicals are not stable enough to survive the multiple steps of polymerizing the different monomers and the purification steps in between. Without chain termination, the living polymerization methods are more versatile in the synthetic design. In this sense, our method is more like free radical polymerization than living polymerization.

In comparison, an ideal method for "polymerizing" NPs has at least two requirements: (a) the end of the "polymer" chain should remain active during the 
"polymerization" and purification steps; and (b) the monomers should not "polymerize" on their own. An alternative approach is to somehow selectively re-activate the "polymers" after purification, in order to allow the subsequent growth. These requirements are difficult to meet, because NPs typically have very similar reactivity in their aggregation. In our system, because the PSPAA shells play a critical role in controlling the NP aggregation, improving the selectivity is still possible by designing or pre-treating the PSPAA shells.

In "polymerizing" magnetic NPs, a special situation arises because the magnetic coupling is always active. One can first make magnetic chains, purify them, and then use them for growing a different kind of monomers. The main challenge here is to selectively separate the block "copolymers" as shown in figure 6.14 from the "homopolymers". Alternatively, it is also possible to first make homopolymers of A and B separately, and then couple them together into block "polymers",23. Due to the difficulty in the specific A-B coupling, random coupling of the chains would give many combinations, including $\mathrm{AB}, \mathrm{AA}, \mathrm{BB}$, $\mathrm{AAB}, \mathrm{ABB}, \mathrm{BAB}, \mathrm{ABA}$, etc. Also because of this problem, simply mixing monomers of $\mathrm{A}$ and $\mathrm{B}$ is not an option, as it would only give random “copolymers".

\subsection{Conclusion}

In summary, we exploited an unconventional assembly system for the "copolymerization" of nanoparticles into chain structures. The unique controls 
by the polymer shells and the chain growth "polymerization" mode are of critical importance for achieving the orderliness of the assembly, namely the ultralong chains with uniform width and few branches. Our understandings of the reaction parameters allowed the generic use of different nanoparticles, nanorods, and nanowires in the assembly. Further successes have been achieved in the hetero-assembly of these different types of nanomaterials. Despite the orderly chain morphology, the arrangement of the nanoparticles in the chains is still far from perfect. Specifically, homo- and random- "copolymers" have been synthesized successfully, but the block-“copolymers" were only partially successful, using a single nanowire as a "block". The key problem was identified as the lack of selectivity in activating the first step "polymers" for growing the second block chains.

Here, we developed a new method to synthesize 1D nanoparticle chains and 3D nanoclusters under the ultrasound. The length of the single-line chains fabricated via the sphere-to-cylinder transformation of PSPAA shells was not uniform (10-300 NPs in length). Under ultrasonic irradiation, the cavitations can caused the scission of PSPAA shells, thus, the ultralong AuNP chains were sectioned into short ones. The average length of the chains and the breakage rate were dependent on the ultrasonic energy. It is found the single-line chains could transform into spherical nanoclusters under a higher ultrasonic energy. The formation of 3D nanoclusters involved the scission and the cylinder-to-sphere 
transformation of PSPAA shells. We hypothesized the mobility of surface ligands on the AuNPs play a critical role in realizing the transformation from chains to clusters. Exploiting the structural transformation of polymer shells under the ultrasound provides a tool to design advanced nanostructures.

Nanoparticle assembly is an emerging field, where the synthetic capability is the main thrust for exploring new properties and new applications. Given the mature field of polymer chemistry and physics, borrowing its concepts and methodology would be a convenient route for enriching the structural variety of nanoparticle assemblies, which would be essential for the future exploration. 


\subsection{Bibliography}

(1). Sau, T. K.; Murphy, C. J., Langmuir 2005, 21, 2923-2929.

(2). Lo, P. K.; Altvater, F.; Sleiman, H. F., J. Am. Chem. Soc. 2010, 132, 10212-10214.

(3). Buck, M. R.; Schaak, R. E., Angew. Chem. Int. Ed. 2013, 52, 6154-6178.

(4). Liu, K.; Lukach, A.; Sugikawa, K.; Chung, S.; Vickery, J.; Therien-Aubin, H.;

Yang, B.; Rubinstein, M.; Kumacheva, E., Angew. Chem. 2014, 126, 2686-2691.

(5). Buck, M. R.; Bondi, J. F.; Schaak, R. E., Nat. Chem. 2012, 4, 37-44.

(6). Sra, A. K.; Ewers, T. D.; Xu, Q.; Zandbergen, H.; Schaak, R. E., Chem. Commun. 2006, 750-752.

(7). Chen, G.; Wang, Y.; Tan, L. H.; Yang, M.; Tan, L. S.; Chen, Y.; Chen, H., J. Am. Chem. Soc. 2009, 131, 4218-4219.

(8). Wang, X. J.; Li, G. P.; Chen, T.; Yang, M. X.; Zhang, Z.; Wu, T.; Chen, H. Y., Nano Lett. 2008, 8, 2643-2647.

(9). Xing, S.; Tan, L. H.; Yang, M.; Pan, M.; Lv, Y.; Tang, Q.; Yang, Y.; Chen, H., J. Mater. Chem. 2009, 19, 3286-3291.

(10). Yang, M. X.; Chen, G.; Zhao, Y. F.; Silber, G.; Wang, Y.; Xing, S. X.; Han, Y.; Chen, H. Y., Phys. Chem. Chem. Phys. 2010, 12, 11850-11860.

(11). DeVries, G. A.; Brunnbauer, M.; Hu, Y.; Jackson, A. M.; Long, B.; Neltner, B. T.; Uzun, O.; Wunsch, B. H.; Stellacci, F., Science 2007, 315, 358-361.

(12). DeVries, G. A.; Talley, F. R.; Carney, R. P.; Stellacci, F., Adv. Mater. 2008, 
$20,4243-4247$.

(13). Zhang, S. Z.; Kou, X. S.; Yang, Z.; Shi, Q. H.; Stucky, G. D.; Sun, L. D.; Wang, J. F.; Yan, C. H., Chem. Commun. 2007, 1816-1818.

(14). Tang, Z. Y.; Kotov, N. A.; Giersig, M., Science 2002, 297, 237-240.

(15). Butter, K.; Bomans, P. H. H.; Frederik, P. M.; Vroege, G. J.; Philipse, A. P., Nat. Mater. 2003, 2, 88-91.

(16). Xia, H.; Su, G.; Wang, D., Angew. Chem. Int. Ed. 2013, 52, 3726-3730.

(17). Zhao, N.; Liu, K.; Greener, J.; Nie, Z.; Kumacheva, E., Nano Lett. 2009, 9, 3077-3081.

(18). Sun, Z.; Ni, W.; Yang, Z.; Kou, X.; Li, L.; Wang, J., Small 2008, 4, $1287-1292$.

(19). Shen, X.; Chen, L.; Li, D.; Zhu, L.; Wang, H.; Liu, C.; Wang, Y.; Xiong, Q.; Chen, H., ACS Nano 2011, 5, 8426-8433.

(20). Wang, H.; Chen, L. Y.; Shen, X. S.; Zhu, L. F.; He, J. T.; Chen, H. Y., Angew. Chem. Int. Ed. 2012, 51, 8021-8025.

(21). Kim, B. Y.; Shim, I.-B.; Monti, O. L. A.; Pyun, J., Chem. Commun. 2011, 47, 890-892.

(22). Liu, K.; Nie, Z. H.; Zhao, N. N.; Li, W.; Rubinstein, M.; Kumacheva, E., Science 2010, 329, 197-200.

(23). Korth, B. D.; Keng, P.; Shim, I.; Bowles, S. E.; Tang, C.; Kowalewski, T.; Nebesny, K. W.; Pyun, J., J. Am. Chem. Soc. 2006, 128, 6562-6563. 
(24). Keng, P. Y.; Shim, I.; Korth, B. D.; Douglas, J. F.; Pyun, J., ACS Nano 2007, $1,279-292$.

(25). Chong, W. H.; Chin, L. K.; Tan, R. L. S.; Wang, H.; Liu, A. Q.; Chen, H., Angew. Chem. Int. Ed. 2013, 8570-8573.

(26). Zhang, L. F.; Yu, K.; Eisenberg, A., Science 1996, 272, 1777-1779.

(27). Zhang, L.; Eisenberg, A., Macromolecules 1996, 29, 8805-8815.

(28). Liu, C. C.; Chen, G.; Sun, H.; Xu, J.; Feng, Y. H.; Zhang, Z.; Wu, T.; Chen, H. Y., Small 2011, 7, 2721-2726.

(29). Zhang, L.; Eisenberg, A., Macromolecules 1999, 32, 2239-2249.

(30). Chen, H. Y.; Abraham, S.; Mendenhall, J.; Delamarre, S. C.; Smith, K.; Kim, I.; Batt, C. A., ChemPhysChem 2008, 9, 388-392.

(31). Wang, H.; Chen, L.; Feng, Y.; Chen, H., Acc. Chem. Res. 2013, 46, 1636-1646.

(32). Liu, C.; Yao, L.; Wang, H.; Phua, Z. R.; Song, X.; Chen, H., Small 2014, 10, 1332-1340.

(33). Chen, L.; Shen, H. W.; Eisenberg, A., J. Phys. Chem. B 1999, 103, 9488-9497.

(34). Zhang, L. F.; Eisenberg, A., J. Am. Chem. Soc. 1996, 118, 3168-3181.

(35). Wang, Y.; Chen, G.; Yang, M. X.; Silber, G.; Xing, S. X.; Tan, L. H.; Wang, F.; Feng, Y. H.; Liu, X. G.; Li, S. Z., et al., Nat. Commun. 2010, 1, 87.

(36). Chen, G.; Wang, Y.; Yang, M. X.; Xu, J.; Goh, S. J.; Pan, M.; Chen, H. Y., J. 
Am. Chem. Soc. 2010, 132, 3644-3645.

(37). Yang, M. X.; Chen, T.; Lau, W. S.; Wang, Y.; Tang, Q. H.; Yang, Y. H.; Chen, H. Y., Small 2009, 5, 198-202.

(38). Chen, T.; Wang, H.; Chen, G.; Wang, Y.; Feng, Y.; Teo, W. S.; Wu, T.; Chen, H., ACS Nano 2010, 4, 3087-3094.

(39). Feng, Y. H.; Wang, Y.; Wang, H.; Chen, T.; Tay, Y. Y.; Yao, L.; Yan, Q. Y.; Li, S. Z.; Chen, H. Y., Small 2012, 8, 246-251.

(40). Azzam, T.; Eisenberg, A., Angew. Chem. In. Ed. 2006, 45, 7443-7447.

(41). Terreau, O.; Luo, L. B.; Eisenberg, A., Langmuir 2003, 19, 5601-5607.

(42). Zhang, L. F.; Eisenberg, A., Macromolecules 1996, 29, 8805-8815.

(43). Urban, A. S.; Shen, X.; Wang, Y.; Large, N.; Wang, H.; Knight, M. W.;

Nordlander, P.; Chen, H.; Halas, N. J., Nano Lett. 2013, 13, 4399-4403.

(44). Wang, T.; LaMontagne, D.; Lynch, J.; Zhuang, J.; Cao, Y. C., Chem. Soc. Rev. 2013, 42, 2804-2823.

(45). Mai, Y. Y.; Eisenberg, A., Chem. Soc. Rev. 2012, 41, 5969-5985.

(46). Discher, D. E.; Eisenberg, A., Science 2002, 297, 967-973.

(47). Cui, H.; Chen, Z.; Wooley, K. L.; Pochan, D. J., Soft Matter 2009, 5, $1269-1278$.

(48). Lin, Z. H.; Yang, Z. S.; Chang, H. T., Crys. Growth Des. 2008, 8, 351-357.

(49). Tan, L. H.; Xing, S. X.; Chen, T.; Chen, G.; Huang, X.; Zhang, H.; Chen, H. Y., ACS Nano 2009, 3, 3469-3474. 
(50). Sau, T. K.; Rogach, A. L. Adv. Mater. 2010, 22, 1781

(51). Burke, S. E.; Eisenberg, Langmuir 2001, 17, 6705-6714.

(52). Wang, Y.; He, J.; Liu, C.; Chong, W. H.; Chen, H., Angew. Chem. Int. Ed., 2014. DOI:10.1002/anie.201402986.

(53). Chen, L. Y.; Wang, H.; Xu, J.; Shen, X. S.; Yao, L.; Zhu, L. F.; Zeng, Z. Y.; Zhang, H.; Chen, H. Y., J. Am. Chem. Soc. 2011, 133, 9654-9657.

(54). Braunecker, W. A.; Matyjaszewski, K., Prog. Polym. Sci. 2007, 32, 93-146.

(55). Hill, L. J.; Bull, M. M.; Sung, Y.; Simmonds, A. G.; Dirlam, P. T.; Richey, N. E.; DeRosa, S. E.; Shim, I.-B.; Guin, D.; Costanzo, P. J., et al., ACS Nano 2012, 6, 8632-8645.

(56). Hill, L. J.; Richey, N. E.; Sung, Y.; Dirlam, P. T.; Griebel, J. J.; Lavoie-Higgins, E.; Shim, I.-B.; Pinna, N.; Willinger, M.-G.; Vogel, W., et al., ACS Nano 2014, 8, 3272-3284.

(57). Frens, G, Nat. Phys. Sci. 1973, 241, 20-22.

(58). Gole, A.; Murphy, C. J, Langmuir 2008, 24, 266-272.

(59) X. H. Song, H. Wang, C. C. Liu, JT. He, W. H. Chong, H. Y. Chen, ACS Nano, 2014, 8 (8), 8063-8073. 


\section{Chapter 7 Summary and Outlook}

\subsection{Summary}

Silica is a hot topic from the day when it has been found for the broad application in many different areas, such as biosensor, drug delivery, catalyst, as well as battery. The properties which make silica so hot are its porous shell, large surface area, high stability, and good compatibility. However, the real control based on the silica synthesis procedure is not developed well, because people still do not realize what had happened during the silica formation, including the following issues which have not been solved: why ammonia is necessary for silica formation? Is there any other function for ammonia besides being used as catalyst to TEOS hydrolysis? Why organic solvent is necessary (methanol, ethanol, or isopropanol) during the Stöber silica formation? Where does the silica selective dissolving come from while as-synthesized silica was etched in neutral DI water? The understanding of these problems is important to study the Stöber silica formation mechanism.

For the polymer coating metal nanoparticles the polymerization process control is very important to give different kinds of nanostructures, such as core-shell nanoparticles, dumbbell nanoparticles, Janus nanoparticles, and so on. Under the interfacial energy control we give novel metal NP@PSPAA nanostructures which are critical to future design and application. By tuning 
different parameters we learn the mechanism of polymerization process there.

\subsubsection{Silica formation mechanism and application}

We argue for a paradigm shift in viewing silica nanomaterials. Silica is an old material with useful applications. It is a covalent network solid well known of its insolubility in water. In contrast to the silica materials underwent molten conditions (sand, glass, etc.), those synthesized by solution methods have drastically different properties, in terms of the microporosity, the incorporated impurities, and the solubility in neutral aqueous solutions. The phenomena are not well recognized, not to mention the underlying reasons. Here, we investigate the fundamental reasons for the hollowing of silica nanoparticles upon etching. We realize that, during the synthesis, silica precursors are essentially crosslinked polyelectrolytes, which can take in lots of counter ions and should be highly swollen by the polar solvents. Most importantly, their solubility should depend on the size/length of the poly(silicic acid), the concentration of the counter ions, and the solvent polarity. Thus, the "silica" that nucleates out at the different stages of synthesis should have different degrees of ion doping, which explains the unusual solubility of the Stöber silica, its microporosity, and the selective hollowing of the nanoparticles. We design a range of experiments to test this hypothesis. By strictly controlling the etching conditions, the extent of silica etching (i.e., silica solubility) is shown to correlate to the amount of residue base present in the solution, to the relative amounts of silica and solvent, and to the 
solvent quality (the water/isopropanol ratio). Hollowing does not occur when the silica nanoparticles are incubated in solutions pre-saturated with "silica", ruling out surface reposition and Ostwald ripening as the hollowing mechanism. The embedded ions in silica are confirmed by elemental analysis (CHNS) and ICP-MS. With this knowledge and the shift from the conventional views, we design different quality of silica by modulating the solvent ratio, acidity, and salt concentration, to control the porosity and solubility of selective silica layers. We also incorporate transition metal ions, cationic dyes and drug molecules inside silica, creating new toolboxes for future applications.

The paradigm shift in viewing silica nanomaterials has led to coherent explanations to the long-standing puzzles regarding to the silica formed by solution methods. We highlight them as questions in the following for easy understanding.

Why $\mathrm{NH}_{3}$ or $\mathrm{NaOH}$ is essential for the silica synthesis? The conventional view is that a base is needed to catalyze the hydrolysis of TEOS and/or condensation of Si-OH groups. However, long-term incubation of TEOS in isopropanol/water mixture does not give proper silica nanoparticles. The $\mathrm{NH}_{4}{ }^{+}$ and $\mathrm{Na}^{+}$ions play critical roles as counter ions, which greatly reduce the solubility of the poly(silicic acid) and thus, its nucleation and growth in solution. 
Why organic solvent (methanol, ethanol, or isopropanol) is essential for the Stöber synthesis? Organic solvent can help dissolve TEOS, which is immiscible with water, in the reaction mixture. It also plays a critical role in reducing the solvent polarity and promoting silica nucleation. As discussed above, the poly(silicic acid) is highly soluble in water; its nucleation requires ion pairing and low solvent polarity. The only exception is the reverse emulsion synthesis, ${ }^{1}$ where silica nanoparticles/shells are formed in the tiny emulsion droplets of water. Given the small volume of water, the concentration of $\mathrm{NH}_{3}$ and TEOS are much higher than those used in the Stöber method.

Why the Stöber silica can form in the presence of base and yet dissolves in $p H$ neutral water? The synthetic solution is less polar (water/isopropanol $=2: 5$ ), contains lots of counter ions, and is saturated with silica. When the resulting silica nanoparticles are dispersed in water, all three factors are utterly changed. The embedded ions are equivalent to a base (Figure 2.4b) when dissolving the silica in a $\mathrm{pH}$ neutral solution.

Why the silica prepared using $\mathrm{NaOH}$ is generally more soluble than those prepared using $\mathrm{NH}_{3}$ ? As shown in Figure 2.4b, with counter ions present, the condensation of $-\mathrm{Si}-\mathrm{O}^{-}$with $-\mathrm{Si}-\mathrm{OH}$ gives off one equivalent of $\mathrm{OH}^{-}$ions. Because $\mathrm{NaOH}$ is a stronger base than $\mathrm{NH}_{3} \cdot \mathrm{H}_{2} \mathrm{O}$, the silica embedded with $\mathrm{Na}^{+}$ ions is generally more soluble. Moreover, as shown in Figure 2.7a-f, the amount of trapped ions is usually higher for $\mathrm{Na}^{+}$than $\mathrm{NH}_{4}{ }^{+}$. 
Why it is hard to block the pores in silica using TEOS or other oxide precursors? A nice way to control the porosity of silica would be to block its pores, which would have great impacts in applications, for example, for retaining toxic $\mathrm{CdSe}$ within silica shells. However, the pores originate from the swelling of the poly(silicic acid) by the polar solvent. Just like a piece of rubber in gasoline, the swollen state is thermodynamically favourable. Blocking the pores would reduce the silica-solvent contact area and thus the solvation energy, making the process energetically uphill.

\subsubsection{Metal NPs@PSPAA design and synthesis}

Growing ultralong chains of nanoparticles is extremely difficult, largely due to the lack of selectivity in nanoparticle aggregation. If the aggregation follows the step growth polymerization mode, the monomers will be quickly consumed, giving large number of dimers, trimers, and then clusters. Unless the aggregation among the large clusters is highly selective (not true except for magnetic nanoparticles), the shape control will be easily compromised, giving globular or branched aggregates.

In the thesis we first study the PSPAA nucleation and deposition on the metal nanoparticle surface by tuning many different parameters during the polymer coating procedure, such as the solvent ratio, the temperature, the $\mathrm{pH}$ of the solvent, as well as the polymer chain length. All the adjustment can affect the polymer swelling, mobility as well as the nanoparticle aggregation. We have 
investigated in detail the driving forces in forming the anisotropic structures by specifically identifying the ligand distribution on the AuNPs surface. The ligand shell was found to have significant phase segregation with $\mathrm{L}_{\mathrm{A}}$ attached to the polymer and $\mathrm{L}_{\mathrm{B}}$ exposed to the solution. The PSPAA shell preferentially attaches on the $\mathrm{L}_{\mathrm{A}}$ regions on the metal core and adapt to the morphology with the lowest overall surface energy. The phase segregation of the mixed ligands may have been promoted in the presence of PSPAA, dynamic exchange of the ligand and PSPAA was observed with anisotropic cores. This dynamic exchange strives to achieve the state with minimal surface energy depending on the ration of $\mathrm{L}_{\mathrm{A}} / \mathrm{L}_{\mathrm{B}}$. This opens up the opportunity to specifically position the patches of ligands by manipulating the attachment of PSPAA. This work has been largely qualitative based on TEM observation which bypasses other more quantitative characterization methods that work for large or flat surfaces.

The second synthesis design is Homo- and Co-Polymerization of Polysytrene-Block-Poly(Acrylic Acid)-Coated Metal Nanoparticles based on the surface charge repulsion control.

In this work, we developed a general method for "polymerizing" nanostructures into ultralong chains, where the basic units include 16 and $32 \mathrm{~nm}$ Au nanoparticles, Au nanorods, Te nanowires, carbon nanotubes, as well as cylindrical and vesicular polymer sections. The trick was to exploit the polymer shells on these nanostructures for achieving the chain growth "polymerization" 
mode. We further explored the "co-polymerization" of these units, achieving orderly shape control, namely the ultralong chains with uniform width and few branches. Homo-, random-, and block- "copolymers" have been synthesized, greatly enriching the structural variety of nanoparticle assemblies.

\subsection{Outlook}

This thesis has provided a good platform for novel nanostructures synthesis based on the understanding of the formation mechanism in depth. The future work would be explored into broad areas for nanostructures design and application. What we should do is try to test the primary possibilities of the further application and design providing for the future study. So here we explore our system in two different areas: (1) we hope to explore ion pairing mechanism to other kinds of metal oxides. What we want to know is the mechanism of metal oxide nanostructures formation and hollowing mechanism. It is critical to the novel structure design and potential application in many different areas; (2) we tune the different experimental parameters during the Au NP@PSPAA to tune the polymer shell porosity which is critical to the polymer application in drug delivery and battery. The Surface-Enhanced Raman Spectroscopy (SERS) test can help to check the change of the polymer shell porosity during the control. According to the porosity control we can get different kinds of polymer shell, and 

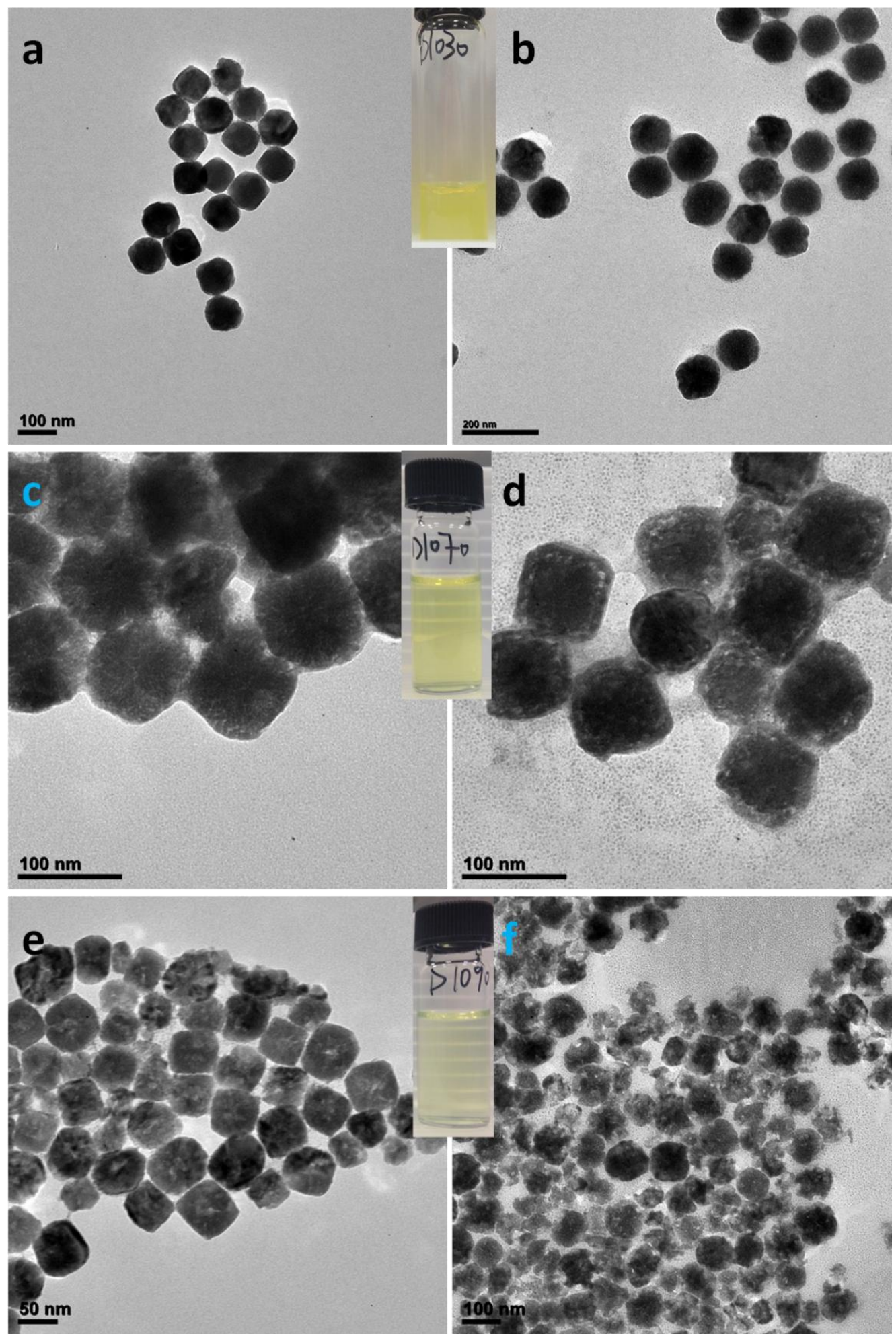

Figure 7.1. TEM images showing the purified products of $\mathrm{Cu}_{2} \mathrm{O}$ nano cubes synthesized at different surfactant PVP concentration of: (a) $2 \mathrm{mg} / \mathrm{mL}$; (b) 4 $\mathrm{mg} / \mathrm{mL}$; (c) $6 \mathrm{mg} / \mathrm{mL}$; (d) $8 \mathrm{mg} / \mathrm{mL}$; (e) $10 \mathrm{mg} / \mathrm{mL}$; (f) $12 \mathrm{mg} / \mathrm{mL}$. It is clear to see heavier hollowing at higher PVP concentration. 


\subsubsection{Ion doping behavior of $\mathrm{Cu}_{2} \mathrm{O}$ nanoparticle}

The experimental is that we synthesize $\mathrm{Cu}_{2} \mathrm{O}$ nanocube firstly, and then coat an amorphous layer of $\mathrm{Cu}_{2} \mathrm{O}$ on the nanocube surface to get the core-shell nanostructure. For the amorphous shell coating procedure we control the $\mathrm{NH}_{4} \mathrm{NO}_{3}$ concentration to see the shell morphologies difference. ${ }^{1}$ All the nanocrystal has been characterized by TEM, SEM, and XRD to confirm the structure here.

To the $\mathrm{Cu}_{2} \mathrm{O}$ nanocube synthesis, we find that the surfactant (PVP) concentration is critical to the final morphologies. PVP concentration increase could lead to the surfactant micelle and adsorption different on the primary nucleation seed surface, which might be embed inside the crystal. ${ }^{2}$ So it is clear to see from the Figure 7.1, which shows that hollow $\mathrm{Cu}_{2} \mathrm{O}$ nanocube was collected at the high concentration of PVP solution during the synthesis. The $\mathrm{Cu}_{2} \mathrm{O}$ nanocube synthesized at the lower PVP concentration of without any PVP addition could be more density. Similarly, the same trend can also be found while SDS was used as the surfactant.

Especially when the surfactant concentration is very high (larger than $8 \mathrm{mg} / \mathrm{mL}$ ) the nanocube would be higher hollow. The HRTEM data in Figure 7.2 show it is mesocrystal. Here this kind of control has never been reported for the $\mathrm{Cu}_{2} \mathrm{O}$ nanocube synthesis.

For the $\mathrm{Cu}_{2} \mathrm{O}$ nanocube coating another spherical layer of $\mathrm{Cu}_{2} \mathrm{O}$, we have try to 
introduce the salt $\left(\mathrm{NH}_{4} \mathrm{NO}_{3}\right)$ into the synthesis, which will help us to know

whether the ions can affect the oxide formation and hollowing just like it does in silica formation process.
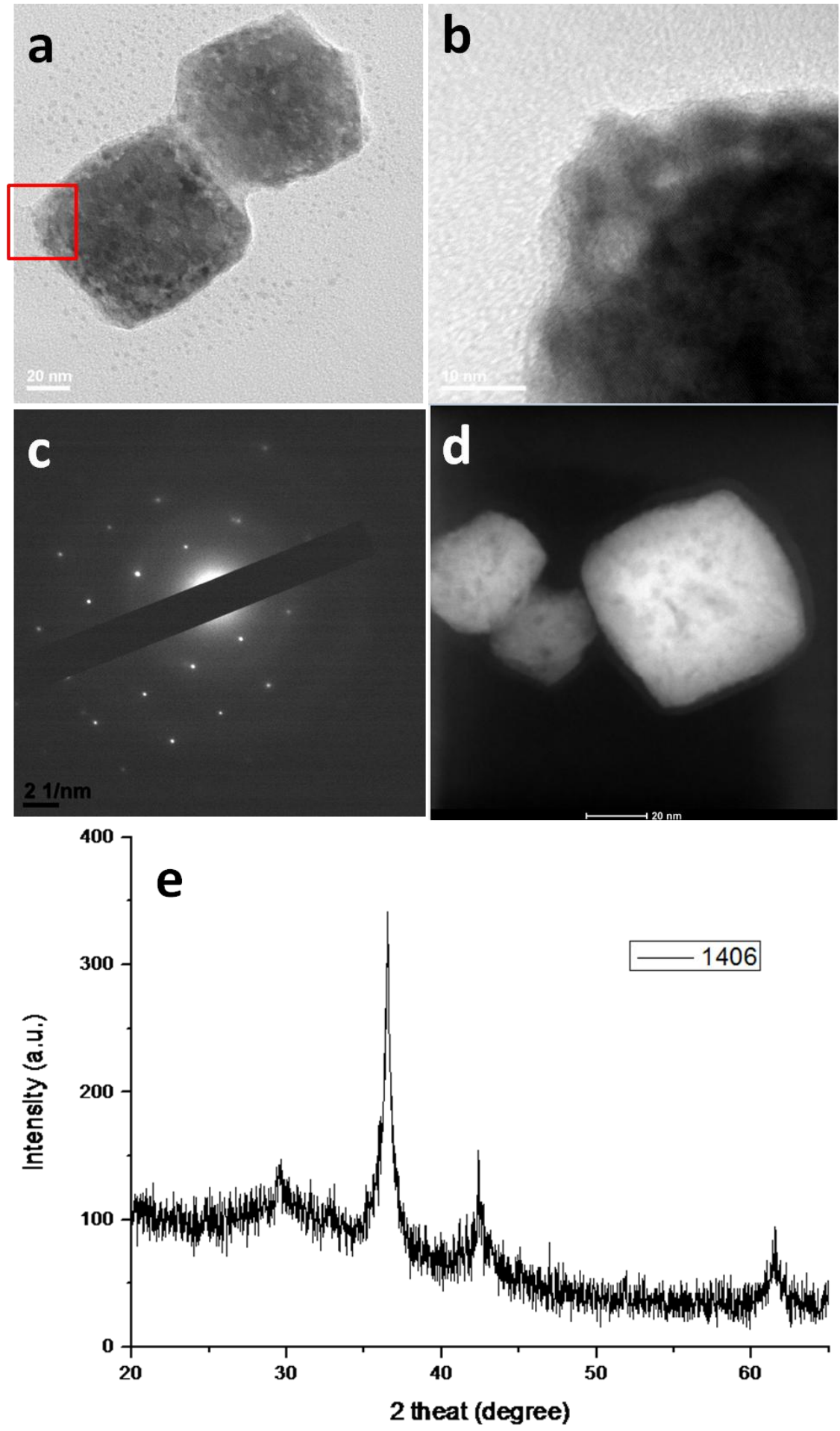

Figure 7.2. HRTEM images showing the purified products of $\mathrm{Cu}_{2} \mathrm{O}$ nano cubes 
synthesized at surfactant PVP concentration of $6 \mathrm{mg} / \mathrm{mL}$ : (a) TEM image of the nanocube; (b) the edge of sample a; (c) the lattice patterning of sample a; (d) the STEM of smaple a; (e) XRD of sample a.
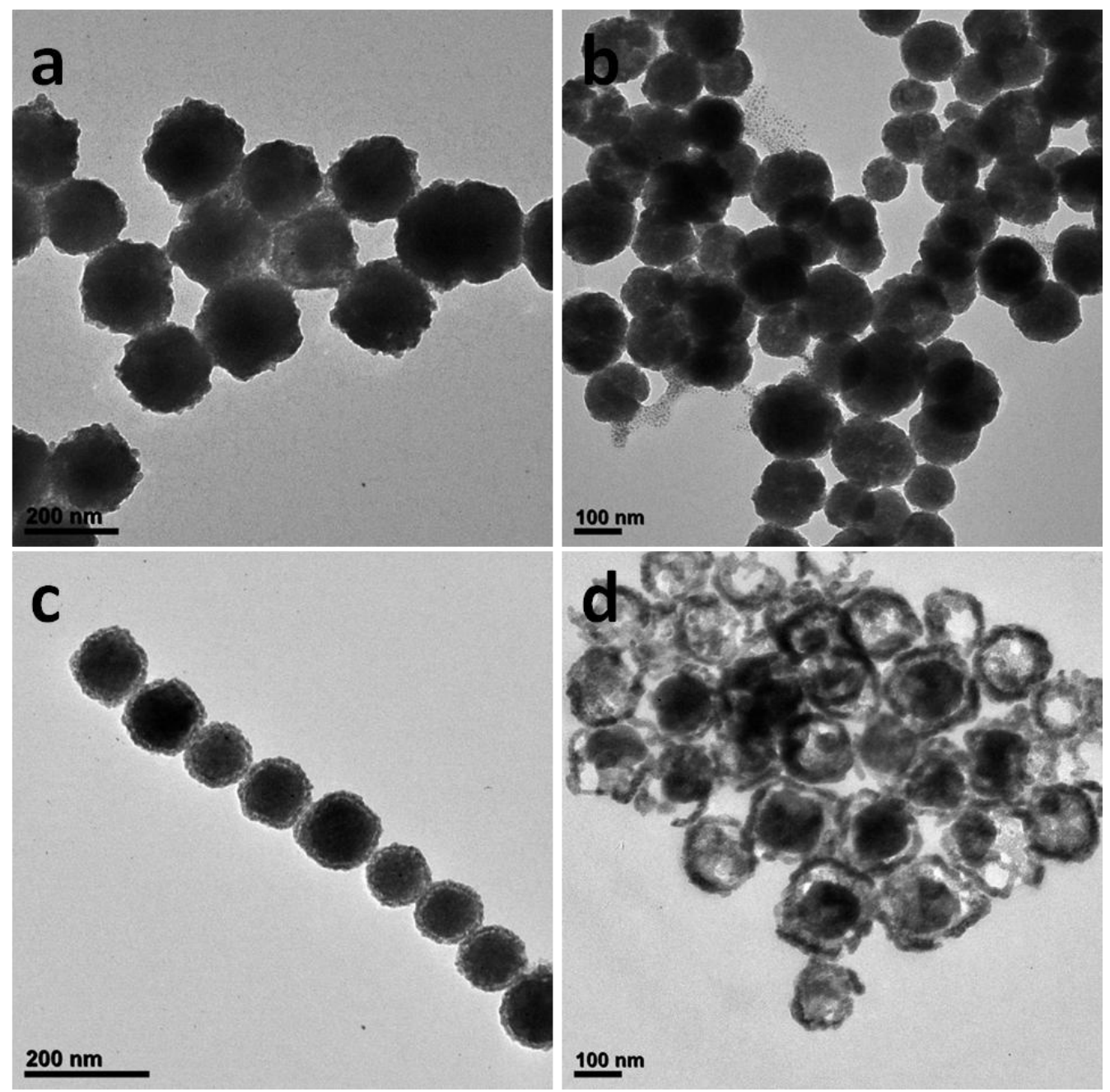

Figure 7.3. TEM images showing the purified products of $\mathrm{Cu}_{2} \mathrm{O}$ nano cubes@

$\mathrm{Cu}_{2} \mathrm{O}$ spherical shell at different salt $\left(\mathrm{NH}_{4} \mathrm{NO}_{3}\right)$ concentration of: (a) 0 ; (b) $2 \mathrm{mM}$;

(c) $4 \mathrm{mM}$; (d) $6 \mathrm{mg} / \mathrm{mL}$; it is clear to see the higher salt concentration gives heaviser hollowing core-shell nanoparticles.

According to Figure 7.3, it is clear to see that the spherical $\mathrm{Cu}_{2} \mathrm{O}$ shell 
morphologies are different at different salt $\left(\mathrm{NH}_{4} \mathrm{NO}_{3}\right)$ concentration. Typically,
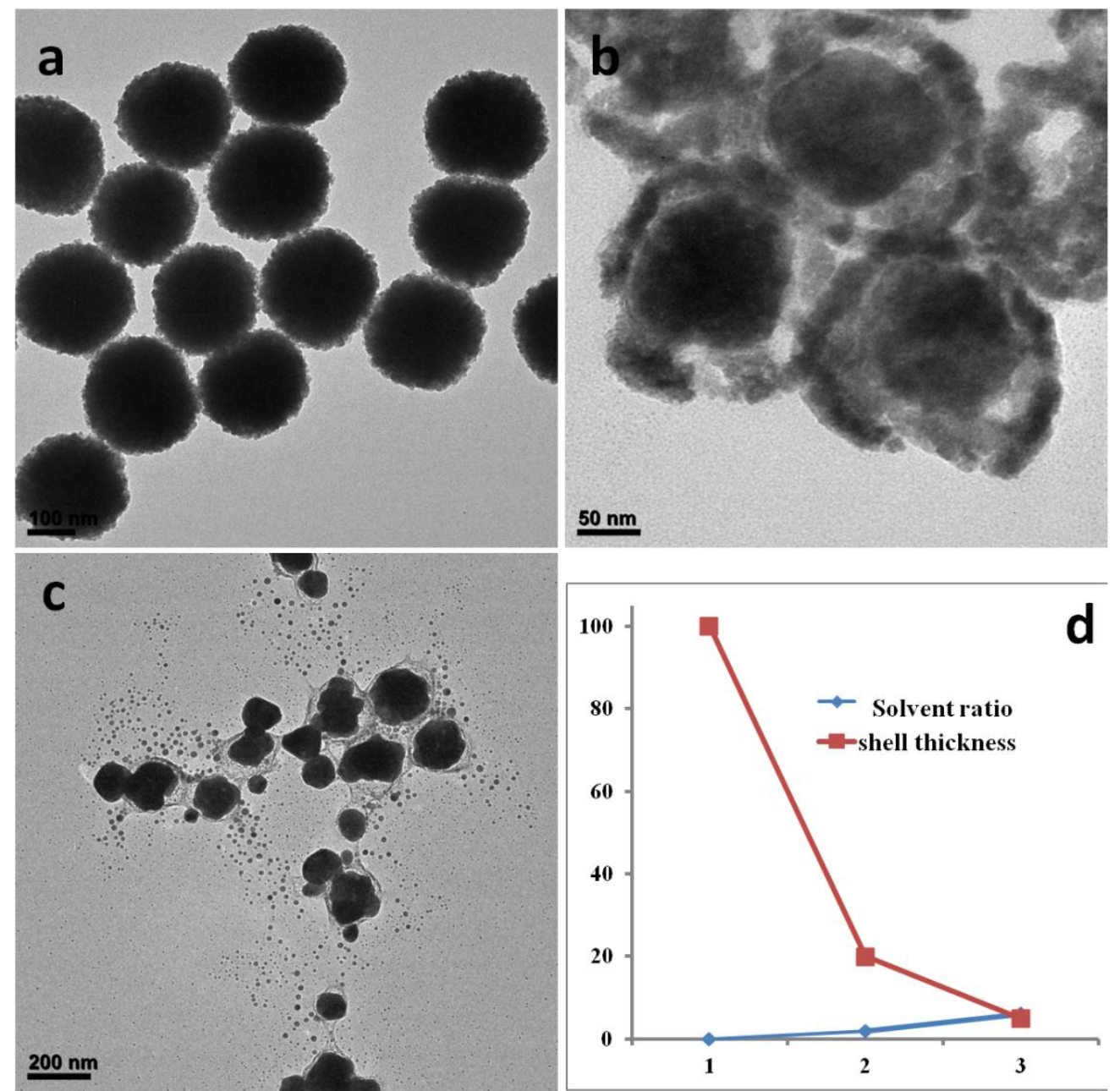

Figure 7.4. TEM images showing the purified products of $\mathrm{Cu}_{2} \mathrm{O}$ nano cubes@ $\mathrm{Cu}_{2} \mathrm{O}$ spherical shell at different solvent ratio of water/EtOH: (a) 1:4; (b) 1:1; (c)

4:1. (d) the shell thickness relationship with the solvent ratio. It is clear to see higher polarity of etching solvent lead to heavier hollowing while giving thinner shell for the $\mathrm{Cu}_{2} \mathrm{O}$ nano cubes@ $\mathrm{Cu}_{2} \mathrm{O}$ spherical nanoparticle.

the core-shell nanoparticle was the final product without any $\mathrm{NH}_{4} \mathrm{NO}_{3}$ addition; it will be lightly hollow at the interface of the nanocube core and the spherical shell while salt $\left(\mathrm{NH}_{4} \mathrm{NO}_{3}\right)$ concentration is $2 \mathrm{mM}$; the heavy hollowing shell 
would be the final product while the salt $\left(\mathrm{NH}_{4} \mathrm{NO}_{3}\right)$ concentration is increased to $6 \mathrm{mM}$. So it is clear to see the trend between the salt $\left(\mathrm{NH}_{4} \mathrm{NO}_{3}\right)$ concentration and the spherical shell morphology there. ${ }^{3}$ We think it is possible that the ion doping caused by the ion concentration difference might affect the shell morphology. ${ }^{4}$ However, we need more evidence to prove the hypothesis in future work.

Another two data support our hypothesis about the ion doping inside the $\mathrm{Cu}_{2} \mathrm{O}$ shell coming from the solvent ratio and BET. The solvent ratio control experiments (Figure 7.4) of the core-shell structure etching show: at lower solvent ratio (water/EtOH $=1: 4$ ) there would be heavier hollow shells after etching; at medium solvent ratio (water/EtOH = 1:1) there would be lightly hollow shell,

The BET data shown in figure 7.5 also help to support our hypothesis. For the $\mathrm{Cu}_{2} \mathrm{O}$ nanoparticles synthesized at the lower salt $\left(\mathrm{NH}_{4} \mathrm{NO}_{3}\right)$ concentration, the BET data show that it has smaller surface area $\left(68.74 \mathrm{~m}^{2} /\right)$; for the $\mathrm{Cu}_{2} \mathrm{O}$ nanoparticles synthesized at the higher salt $\left(\mathrm{NH}_{4} \mathrm{NO}_{3}\right)$ concentration, the BET data show that it has large surface area $\left(83.15 \mathrm{~m}^{2} /\right)$. The result means that at higher salt concentration the $\mathrm{Cu}_{2} \mathrm{O}$ nanoparticles porosity would be higher compared it at the lower salt concentration. It can be explain under the ion doping mechanism which means that the higher salt concentration might lead to heavier ion doping inside the $\mathrm{Cu}_{2} \mathrm{O}$ nanoparticles, which would trap more counter ions inside. Then there would be more defect inside the $\mathrm{Cu}_{2} \mathrm{O}$ nanoparticles leading to 
high porosity. But we still need to test more experiments such as the pore size change, the temperature effects, and so on.
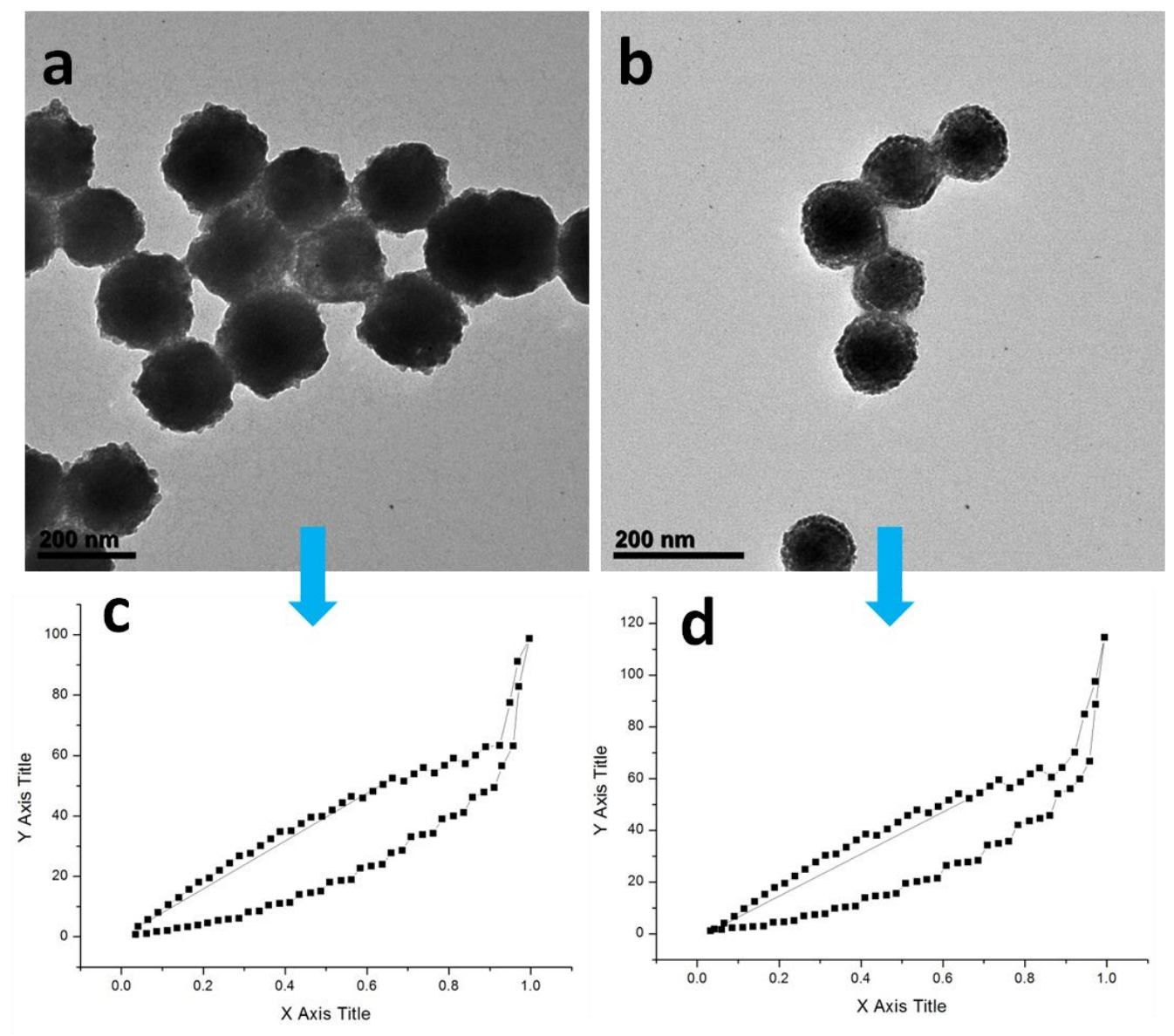

Figure 7.5. TEM images showing the purified products of $\mathrm{Cu}_{2} \mathrm{O}$ nano cubes@ $\mathrm{Cu}_{2} \mathrm{O}$ spherical nanoparticle at different different salt $\left(\mathrm{NH}_{4} \mathrm{NO}_{3}\right)$ concentration of:

(a) 0; (b) $6 \mathrm{mM}$; and the correspounding BET data to show the surface area of the two samples.

It is clear that more study needed to do to support and explore our present ion doping mechanism in other metal oxide nanostructures although the present data strongly consistent with our hypothesis. Besides $\mathrm{Cu}_{2} \mathrm{O}$ nanoparticles, we need to test $\mathrm{ZnO}, \mathrm{Ti}_{2} \mathrm{O}$ and so on. 
Here we indicate that to explore the ion doping mechanism to other oxides formation there is no solid evidence as silica nanoparticle etching study. One reason is that this is a new direction which we will focus on in future; another reason is that this different systems connection study is a long way and we hope this proposed mechanism is just a consideration even though there is no solid evidence. We believe that it is helpful to the hollow mechanism study although it might be incorrect, and there is a word "the success usually comes from many times mistakes by trying'.

\subsubsection{Control polymer shell porosity}

Just like we can control silica shell porosity we hope to control the polymer shell porosity which is critical to potential application (as shown in figure 7.6) in drug delivery, ${ }^{5}$ biosensor, ${ }^{6}$ as well as catalyst.

Based on our previous understanding of AuNP encapsulated by a polystyrene-block-poly(acrylic acid) (PSPAA), we hope to tune the polymer shell porosity by tuning the polymer shell swelling and mobility under different conditions. Some primary study has been done here. ${ }^{7,8}$ We try to use SERS to investigate the cyanide ions diffusion change during the PSPAA shell porosity control. Actually there are many parameters to tune if one wants to control the polymer shell porosity, such as modulating the PS blocks by organic solvent. ${ }^{10}$ 


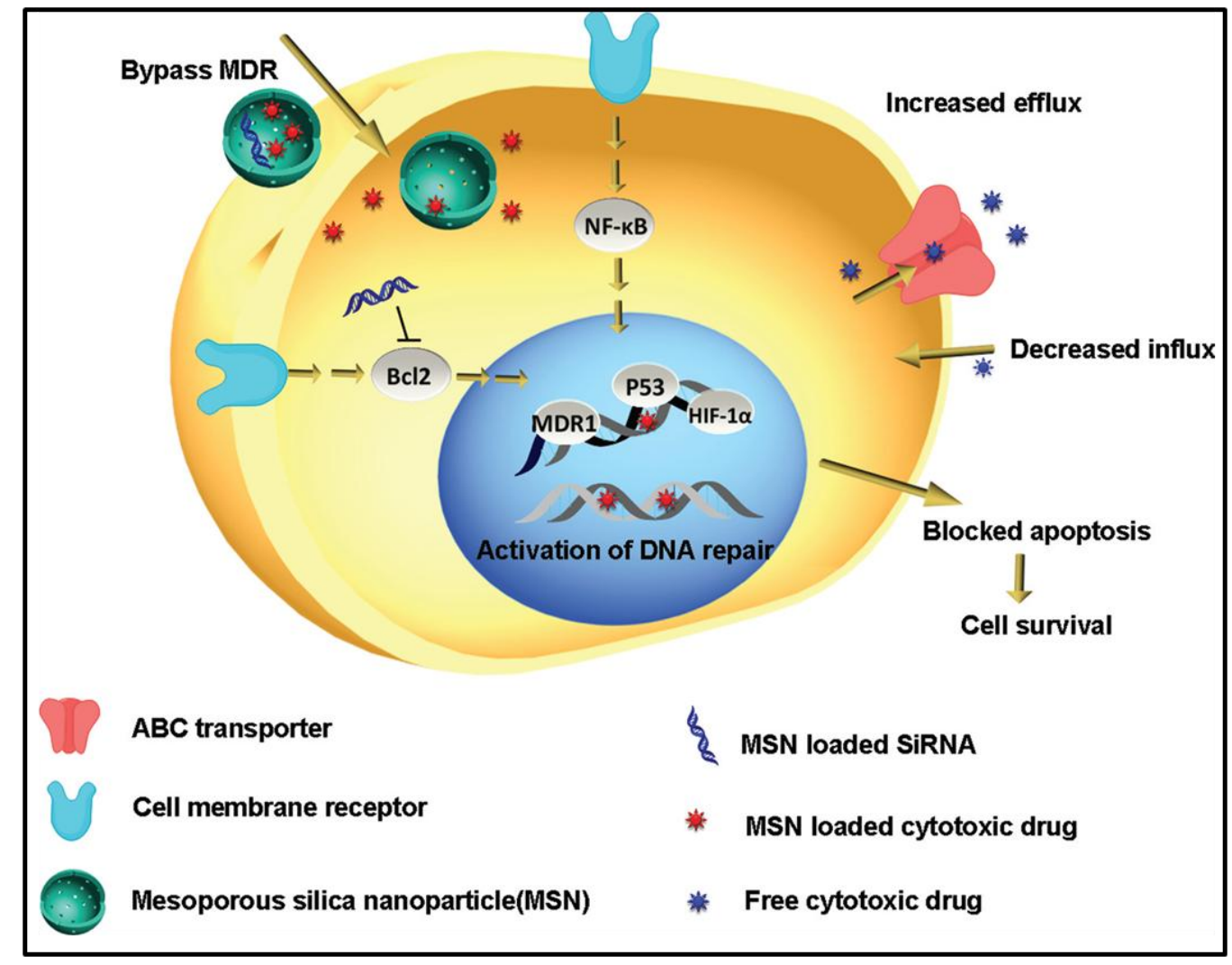

Figure 7.6. Porous nanoparticle used in drug delivery system. Reprinted with permission from ref. 8. Copyright 2008 from American Chemical Society.

Our primary test is just the organic solvent control. What we did is that swelling the PS domain of AuNP@PSPAA by organic solvent, which means that we change the solvent ratio of organic solvent to water while incubating the core-shell nanoparticle inside the mixture. ${ }^{11}$ Here we choose THF as the swelling solvent for three reasons: at first, $\mathrm{KCN}$ is no soluble or react with $\mathrm{THF}$ which means that this can not affect the Au core etching during the incubation process; then another reason is that THF and water can form a miscible liquid with the 


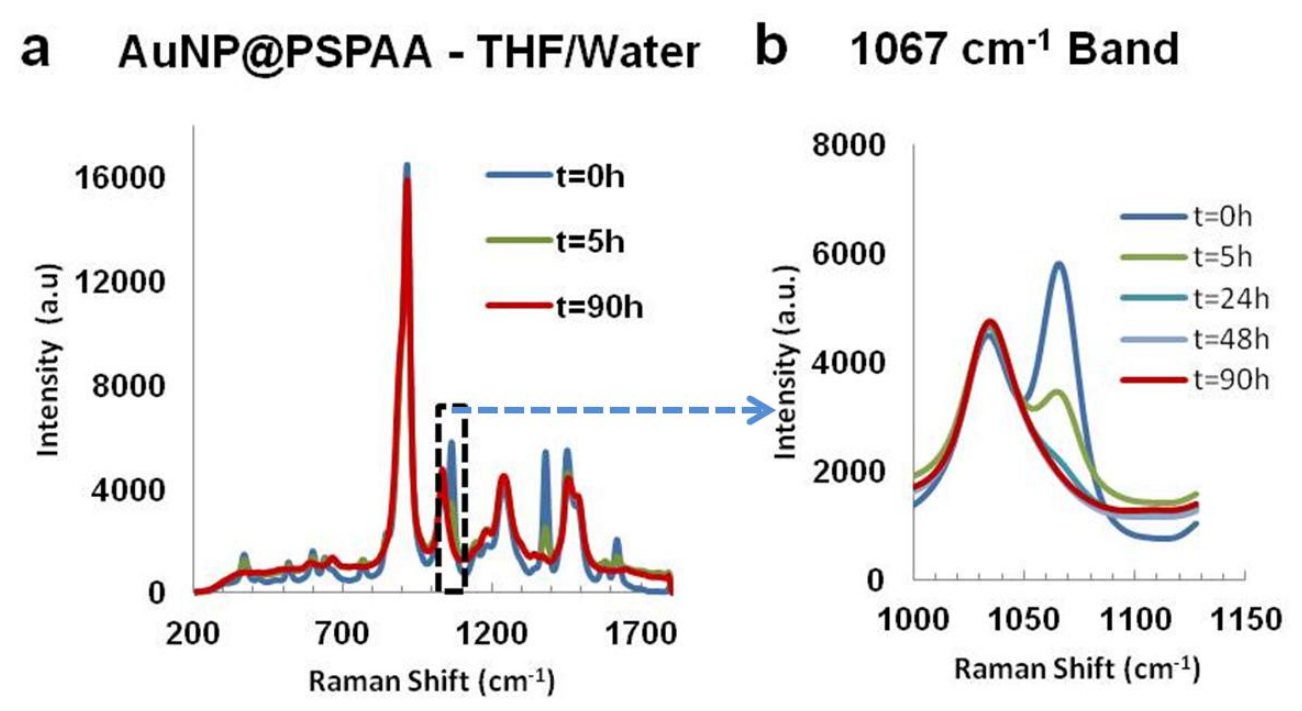

C Etching of AuNP@PSPAA@THF/Water

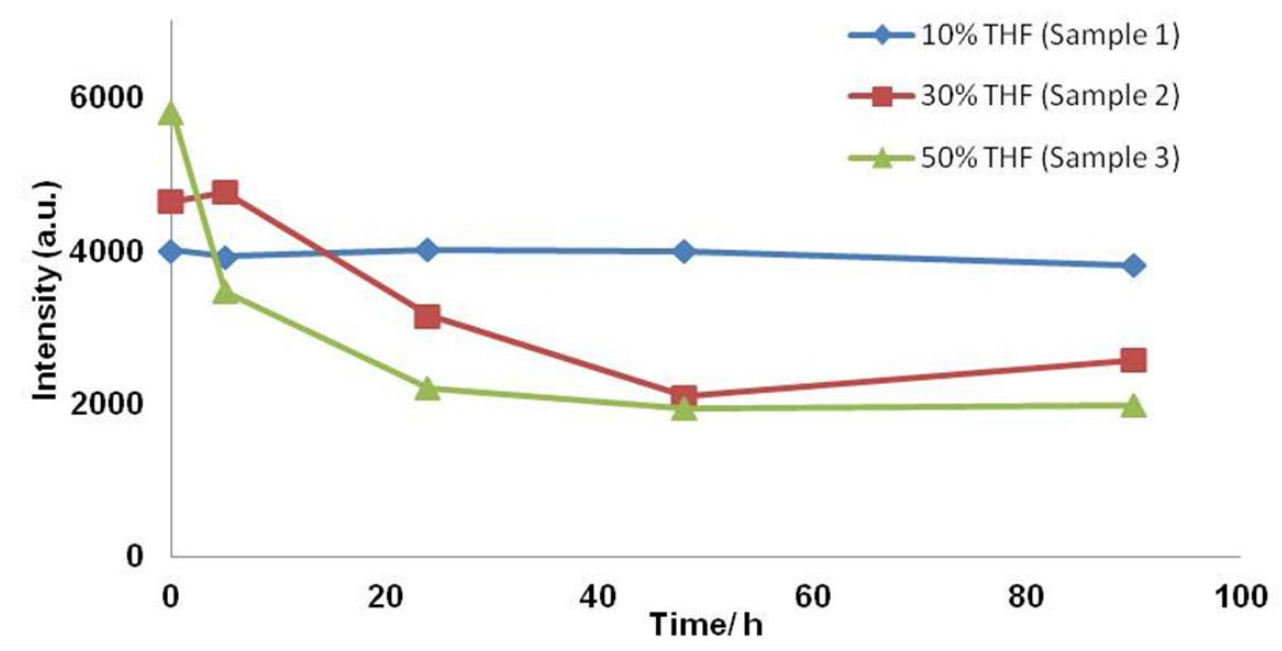

Figure 7.7. Raman spectra of AuNP@PSPAA incuated at the different solvent ratio of $\mathrm{THF} /$ water mixtures solution while etching the $\mathrm{Au}$ core by $\mathrm{KCN}$, the other condition is the same such as the NP concentration, the temperature, and so on.(a) core-shell nanoparticle was etched in $\mathrm{THF} /$ water=1:1 solution; (b) magnified SERS band of (a) at $1067 \mathrm{~cm}^{-1}$ : (c) with increasing amount of THF/water ratio (10,30 and 50\% - Sample 1, 2 and 3 respectively) monitored over $90 \mathrm{~h}$ to check the Raman intensity change during the etching. 
core-shell nanoparticles; finally, THF shows low dielectric constant which makes it distinct to us that during the process of etching, water served as the medium for cyanide ion diffusion. ${ }^{12}$ As shown in Figure 7.7, the solvent ratio change of THF/water (10, 30, $50 \%)$ could really change the SERS intensity, which means that higher THF concentration can make more THF solvent diffuse indie the polymer network to swell polymer. Then the PSPAA shell porosity would increase to let more water and cyanide diffuse inside the core-shell inner layers. The Au core surface etching would be heavier while the SERS intensity must decrease like shown in Figure 7.7.

More future work need to do for better understanding and control of PSPAA shell porosity. This future work is very important to tune a hydrophobic shell or membrane porosity in drug delivery, catalyst, as well as biosensors. ${ }^{13}$

\subsubsection{Design novel structures for catalyst-proposed application.}

Based on our previous study on silica formation mechanism, the silica synthesis control, the silica coating environment study, and polymer coating study, we can synthesis different kinds of hybrid nanostructures such as Janus nanoparticles, core-shell nanoparticles, polymer chains, as well as other kinds of hybrid nanostructures.

In order to design novel nanostructures with high potential application in catalysts such as photo catalysis, electro chemical catalysis. We proposed to coating ultrathin Au nanowire with silica shell, doping many different kinds of 
transition metals, such as $\mathrm{Co}, \mathrm{Ni}, \mathrm{Pb}$, and so on. All these transition metals are good catalysts for electrochemical catalyst.

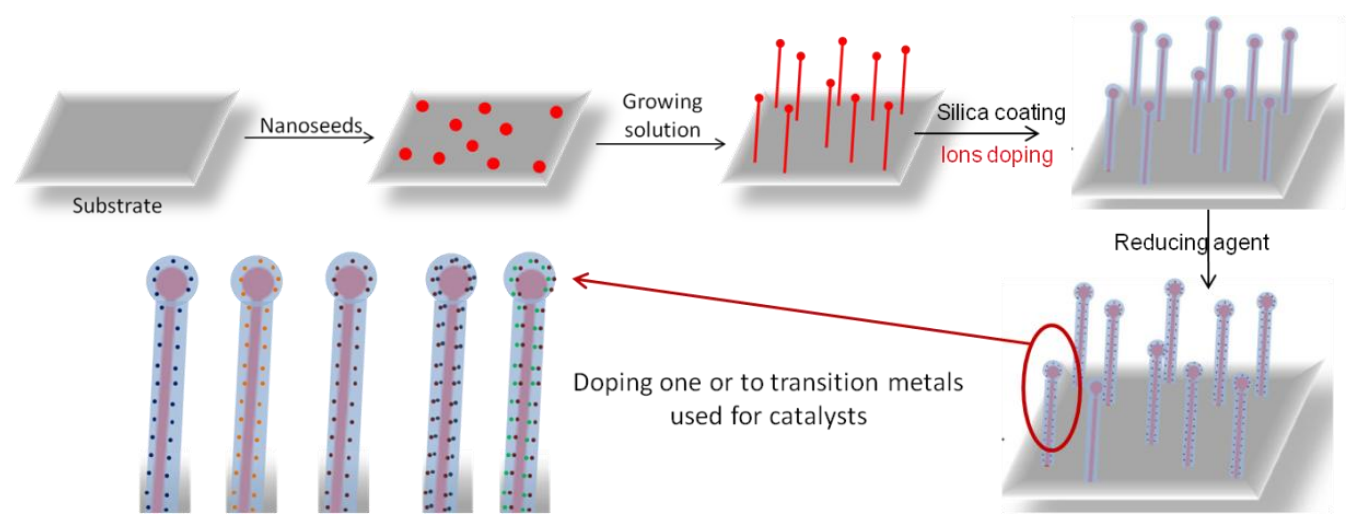

Figure 7.8: schematic illustrates the procedure of ion doping control during silica coating Au nanowires.

As shown in figure 7.8, it is clear to see that with the ion doping during the silica coating of ultrathin Au nanowire we will get novel core-shell nanostructures which contains high quantity of transition metals inside the silica shell. It can be used as catalyst because the silica shell is micro/mesco porous allowing chemical diffusion. Take $\mathrm{Pb}$ doping as an example, when doping $\mathrm{Pb}$ inside $\mathrm{Au}$ nanowire@ silica core-shell nanostructure we can propose that it can be applied to $\mathrm{CO}_{2}$ reduction. Au itself has shown high activity to $\mathrm{CO}_{2}$ reduction compared to other nanomaterials. The over potential might be decreased dramatically while using $\mathrm{Pb}$-doped $\mathrm{Au}$ nanowire as catalyst.

In summary, we believe that our synthetic strategy is critical to future application in catalysts, energy conversion, and drug delivery. 


\subsection{Bibliography}

(1) Ciriminna, R.; Fidalgo, A.; Pandarus, V.; Béland, F.; Ilharco, L. M.;

Pagliaro, M. Chem. Rev. 2013, 113, 6592.

(2) Ahmed, A.; Clowes, R.; Myers, P.; Zhang, H. J. Mater. Chem. 2011, 21, 5753.

(3) Slowing, I. I.; Vivero-Escoto, J. L.; Trewyn, B. G.; Lin, V. S. Y. J. Mater. Chem. 2010, 20, 7924.

(4) Wang, X.; Schröder, H. C.; Wang, K.; Kaandorp, J. A.; Müller, W. E. G. Soft Matter 2012, 8, 9501.

(5) Wong, Y. J.; Zhu, L. F.; Teo, W. S.; Tan, Y. W.; Yang, Y. H.; Wang, C.; Chen, H. Y. J. Amer. Chem. Soc. 2011, 133, 11422.

(6) Fang, X.; Zhao, X.; Fang, W.; Chen, C.; Zheng, N. Nanoscale 2013, 5, 2205.

(7) Chen, Y.; Chen, H. R.; Guo, L. M.; He, Q. J.; Chen, F.; Zhou, J.; Feng, J. W.; Shi, J. L. ACS Nano 2010, 4, 529.

(8) Zhang, T. R.; Ge, J. P.; Hu, Y. X.; Zhang, Q.; Aloni, S.; Yin, Y. D. Angew. Chem.-Int. Ed. 2008, 47, 5806.

(9) Yu, Q. Y.; Wang, P. P.; Hu, S.; Hui, J. F.; Zhuang, J.; Wang, X. Langmuir 2011, 27, 7185 .

(10) Park, S. J.; Kim, Y. J. Langmuir 2008, 24, 12134. 
(11) Chen, S. L.; Dong, P.; Yang, G. H.; Yang, J. J. Industri. \& Eng. Chem. Res. 1996, 35, 4487.

(12) Li, L.; Zhang, L. Y.; Xing, S. X.; Wang, T. T.; Luo, S. R.; Zhang, X. Q.; Liu, C.; Su, Z. M.; Wang, C. G. Small 2013, 9, 825.

(13) Liu, J.; Qiao, S. Z.; Liu, H.; Chen, J.; Orpe, A.; Zhao, D.; Lu, G. Q. Angew. Chem. Int. Ed. 2011, 50, 5947. 


\section{List of Publications}

1. Xiaohui Song, Tao Ding, Lin Yao, Ming Lin, Rachel Lee Siew Tan, Le Yu,

Xiong Wen (David) Lou, and Hongyu Chen*, "On the Origin and Underappreciated Effects of Ion Doping in Silica”, 2015, Small, DOI:smll201500539.

2. Xiaohui Song, Hong Wang, Yuhua Feng, Rachel Lee Siew Tan, Jun Wei, and Hongyu Chen*, Partial Encapsulation in Reverse Emulsion, 2015, Submitted.

3. Xiaohui Song, Jun Xu, Hong Wang, Yong Wang, and Hongyu Chen*, A General Approach for Encapsulating 19 Different Types of Naonparticles in Polystyrene-block-Poly(acrylic acid), 2015, Manuscript preparation.

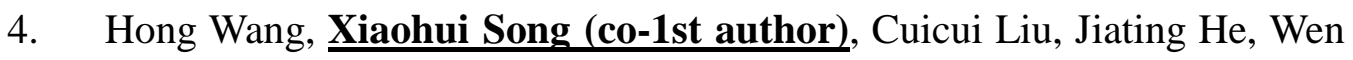
Han Chong, and Hongyu Chen*,"Homo- and Co-polymerization of Nanoparticles", ACS Nano, 2014, 2014, 8 (8), pp 8063-8073.

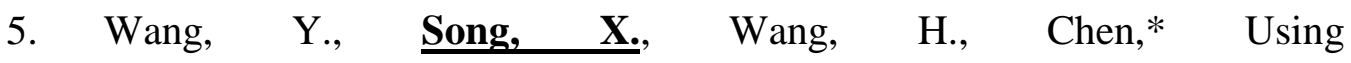
Polystyrene-block-poly(acrylic acid)-coated Metal Nanoparticles as Monomers for Their Homo- and Co-polymerization. J. Vis. Exp.DOI:10.3791/52954 (2015).

6. Rachel Lee Siew Tan, ${ }^{\dagger,} \underline{\text { Xiaohui Song, }}{ }^{\dagger}$ Wen Han Chong, ${ }^{\dagger}$ Yin Fang, ${ }^{\dagger}$ Jun Wei, ${ }^{*}+\stackrel{+}{*}$ and Hongyu Chen ${ }^{*}$, Leveling the Playing Field: Coalesced Bimetallic Nanoparticles for Synergistic Effect in Catalyst Screening, 2015, Nanoscale, accepted.

7. Cuicui Liu, Lin Yao, Hong Wang, Zai Rong Phua, Xiaohui Song, and 
Hongyu Chen*, Small, 2014, Vol 10, Iss 7, pages 1332-1340.

8. X. Y. Yu, Y. Yu, L. F. Shen, X. H. Song, H. Y. Chen, X. W. Lou,*

"General Formation of MS (M=Ni, Cu, Mn) Box-in-Box Hollow Structures with

Enhanced Pseudocapacitive Properties", Advanced Functional Materials, 24, $7440-7446(2014)$

9. Yuhua Feng, Yawen Wang, Jiating He, Xiaohui Song, Yee Yan Tay, Hng Huey Hoon, Xing Yi Ling, and Hongyu Chen* Achieving Site-Specificity in Multistep Colloidal Synthesis, JACS, 2015, accepted 